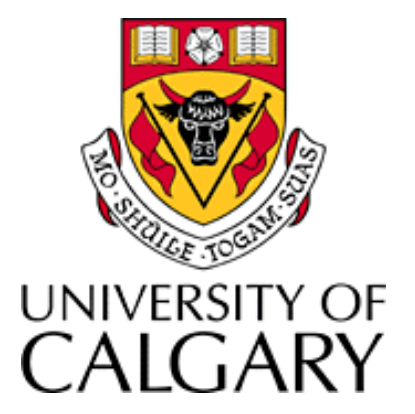

The author of this thesis has granted the University of Calgary a non-exclusive license to reproduce and distribute copies of this thesis to users of the University of Calgary Archives.

Copyright remains with the author.

Theses and dissertations available in the University of Calgary Institutional Repository are solely for the purpose of private study and research. They may not be copied or reproduced, except as permitted by copyright laws, without written authority of the copyright owner. Any commercial use or publication is strictly prohibited.

The original Partial Copyright License attesting to these terms and signed by the author of this thesis may be found in the original print version of the thesis, held by the University of Calgary Archives.

The thesis approval page signed by the examining committee may also be found in the original print version of the thesis held in the University of Calgary Archives.

Please contact the University of Calgary Archives for further information, E-mail: uarc@ucalgary.ca Telephone: (403) 220-7271 Website: http://www.ucalgary.ca/archives/ 
THE UNIVERSITY OF CALGARY

\section{Signal Processing for Optical Fiber Based Wireless Access}

by

Xavier N. Fernando

A THESIS

SUBMITTED TO THE FACULTY OF GRADUATE STUDIES

IN PARTIAL FULFILLMENT OF THE REQUIREMENTS FOR THE DEGREE OF DOCTOR OF PHILOSOPHY

DEPARTMENT OF ELECTRICAL AND COMPUTER ENGINEERING

CALGARY, ALBERTA

DECEMBER, 2001

(c) Xavier N. Fernando 2001 


\section{Abstract}

In this dissertation, signal-processing strategies are investigated for the performance improvement of optical fiber based wireless access schemes. Proposed solutions keep the remote radio access point(RAP) less complex and, do not modify the portable unit keeping the overall system cost low. Furthermore, these deliberations provide asymmetry in complexity and, make seamless roaming between the fiber based wireless systems and conventional wireless systems possible. The scope is limited to single mode radio over fiber (ROF) links and indoor micro/pico cells. Radio frequency is taken as $1.8 \mathrm{GHz}$ and the maximum fiber length is few kilometers.

First part of the dissertation is focused on characterization of the ROF link. This is done by both theoretical and empirical studies. Major limitation of the ROF link is found to be its nonlinearity. This comes from both electrical to optical (E/O) conversion process and amplification.

Then, adaptive nonlinearity compensation schemes are investigated assuming an AWGN wireless channel. Predistortion is done for the downlink and postcompensation is done for the uplink. Both phase and amplitude nonlinearities are handled individually. Simulations based on measured data show adequate compensation is achieved, sometimes with a back off.

Next, the BER performance is analyzed in a CDMA environment, assuming an AWGN wireless channel. An expression is derived for the SNR, which shows the 
inter-relationships between various channel and signal parameters. The BER is then evaluated with matched filter detection.

Thereafter, an algorithm is developed to estimate the concatenated fiber-wireless channel considering multipath dispersion as well. The algorithm estimates linear and the nonlinear parts individually. Numerical evaluation, using real data, shows very good estimation with just a few training symbols.

As the major contribution of this dissertation, a Hammerstein type decision feedback equalizer (HDFE) is proposed for compensating Wiener type nonlinear channels. Although the motivation is triggered by the fiber-wireless uplink, the equalizer is effective against any general Wiener type nonlinear channel. BER performance of the HDFE in this nonlinear channel is very close to that of a DFE in a linear channel under similar conditions. 


\section{Acknowledgements}

I would like to express my sincere acknowledgement to my supervisor Dr. Abu B. Sesay. This work would not have been possible without his matured supervision that is enriched with constant encouragement, timely guidance, plenty of freedom and immense understanding. He is always a considerate mentor and a person of moral support in addition to being an academic supervisor. He even helped a lot to improve my writing skills.

I would also like to thank my supervising committee members Dr. Abraham Fapojuwo and Dr. Dave Irvine-Halliday for their extended support. It is not an exaggeration if I say; I gained most of the knowledge required to research on the optical side of the dissertation from Dr. Dave Halliday. On the other hand, my involvement with Dr. Fapojuwo helped me to improve my organizational skills. He is a very careful reader and a good critic.

During the last four years, the TRLabs provided me with an ideal environment for doing research, in terms of computing as well as high-end lab equipment.

Thanks to the University of Calgary, especially to the Department of Electrical and Computer Engineering and all of its employees who provided me invaluable help and services during my stay here. Special thanks to Ella Gee; she is very well organized in administrative responsibilities.

A very important issue for a graduate student is financial matters. I am im- 
mensely grateful to TRLabs, Natural Science and Engineering Research Council (NSERC) as well as to Informatics Circle of Research Excellence ( $i$ CORE) for providing me with excellent financial support so that I could focus on research. All my conference papers would not have been possible without the travel grants from TRLabs.

I would also like to thank Anthony Lo, Simon Arseneault and Jason Yu for their help in computing and software issues. Thanks to Patrice Opere, Shuwe Huang, Naga Mudigonda and other friends for their numerous small helps and moral support.

Last, but not least I thank to my family, which includes, my dedicated wife Ruby who took care of most of the house-hold responsibilities during this period, relatively nice daughters Christma and Jonna and helpful in-laws who stayed with us for some time. 


\section{Table of Contents}

Approval Page $\quad$ ii

Abstract

Acknowledgements

Table of Contents vii

List of Tables $\quad$ xii

List of Figures $\quad$ xiii

List of Abbreviations $\quad$ xxi

1 Motivation and Background 1

1.1 The Evolution of Wireless Communications. . . . . . . . . . . . 1

1.1.1 The Bandwidth Quest ................ . 2

1.2 Indoor Micro/Pico Cells . . . . . . . . . . . . . . . . . . . 3

1.2.1 The Radio Access Point (RAP) . . . . . . . . . . . 4

1.2 .2 Asymmetry in Complexity . . . . . . . . . . . . 5

1.3 Optical Fiber Based Wireless Access . . . . . . . . . . . . 5

1.3.1 Baseband vs Subcarrier Transmission . . . . . . . . . . . . 6

1.3 .2 A Two Stage Modulation . . . . . . . . . . . . . . . . 7

1.3.3 External and Direct Modulation . . . . . . . . . . . . . 8

1.3.4 Major Limitations . . . . . . . . . . . . . . . . 10

1.4 Previous Attempts to Reduce NLD . . . . . . . . . . . . . . 11

1.4.1 Automatic Gain Controllers and Attenuators . . . . . . . . . 11

1.4.2 Fixed Electronic Solutions . . . . . . . . . . . . . . 12

1.4.3 Network Layer Approaches . . . . . . . . . . . . . . . . . . 12

1.4.4 Miscellaneous Attempts . . . . . . . . . . . . . . . 13

1.4.5 What is Missing? . . . . . . . . . . . . . . . . . . 14

1.5 Major Contrbutions of the Dissertation . . . . . . . . . . . . 15

1.5.1 Baseband Modeling . . . . . . . . . . . . . . 16

1.5.2 Adaptive Predistortion . . . . . . . . . . . . . . . 16 
1.5.3 Adaptive Post-Compensation . . . . . . . . . . . . . . 16

1.5.4 Asymmetry In Complexity . . . . . . . . . . . . . . . . . . . 17

1.5.5 A Joint Solution . . . . . . . . . . . . . . . . . 17

1.5.5.1 Estimation of the Fiber-Wireless Channel . . . . . 17

1.5.5.2 A Hammerstein type Decision Feedback Equalizer . 18

1.6 Organization ......................... 19

2 The Radio Over Fiber Link $\quad 21$

2.1 The Semiconductor Laser . . . . . . . . . . . . . . . . . . . . . . . 21

2.1.1 Nonlinearity of the Laser . . . . . . . . . . . . . . . . . 22

2.1.1.1 Characterization of the Nonlinearity . . . . . . . . 23

2.1.1.2 Static and Dynamic Nonlinearity . . . . . . . . 24

2.1.2 Laser Clipping . . . . . . . . . . . . . . . . . . . . . . 26

2.1.3 Natural Frequency . . . . . . . . . . . . . . . 27

2.1.4 Small Signal Analysis . . . . . . . . . . . . . . . . . . . . . 27

2.1.4.1 Small Signal Frequency Response . . . . . . . . . . 28

2.1.5 Large Signal Analysis . . . . . . . . . . . . . . . . . . . . 28

2.1.6 Laser Chirp . . . . . . . . . . . . . . . . . . . . 29

2.1.7 Relative Intensity Noise (RIN) . . . . . . . . . . . . . . 30

2.1.8 Impedance Matching . . . . . . . . . . . . . . . . . . . . . 30

2.2 Fiber Dispersion and Distortion Issues . . . . . . . . . . . . . . . . . . . . . 31

2.2.1 Chromatic Dispersion . . . . . . . . . . . . . . 31

2.2.2 Polarization Mode Dispersion (PMD) . . . . . . . . . . . . 34

2.2 .3 Interferometric Noise (IN) . . . . . . . . . . . . . . . . 35

2.3 Optical Receiver . . . . . . . . . . . . . . . . . . . . . . . . . . 35

2.3.1 PIN vs Avalanche Photo Diode . . . . . . . . . . . . . . 36

2.3.2 Shot Noise . . . . . . . . . . . . . . . . . . 36

2.3.3 Thermal Noise . . . . . . . . . . . . . . . . 37

2.3.4 Dark Current Noise . . . . . . . . . . . . . . . . . . 37

2.4 Power Link Budget . . . . . . . . . . . . . . . . . . . . . . . . 37

2.5 Preliminary Simulations . . . . . . . . . . . . . . . 38

2.5.1 Optical Transmitter . . . . . . . . . . . . . . . . . . . . . 40

2.5.2 The Fiber-Wireless Link . . . . . . . . . . . . . . . . 41

3 Empirical Characterization 44

3.1 Basic Nonlinearity Measurements . . . . . . . . . . . . . . . . . 45

3.1.1 Effects of Fiber Dispersion . . . . . . . . . . . . . . . . . . . 45

3.1.2 Frequency Response ................. 46

3.1.3 AM-AM and AM-PM Measurements . . . . . . . . . . . . . 49

3.1 .4 Two-Tone Test . . . . . . . . . . . . . . . . . . 51

3.1.5 Noise Measurements . . . . . . . . . . . . . . . . . 52

3.2 Dynamic Measurements . . . . . . . . . . . . . . . 53

3.2.1 Constellation Measurements . . . . . . . . . . . 53 
3.2 .2 Interference Measurements . . . . . . . . . . . . . 55

3.2.2.1 Effect of Interfering Power . . . . . . . . . . 55

3.2.2.2 Effect of Interfering Frequency . . . . . . . . . 56

3.2.3 Power Density Spectrum . . . . . . . . . . . . . 58

3.3 Deductions from Measurements, Simulations and Analysis . . . . . 61

4 Adaptive Modeling and Nonlinearity Compensation 63

4.1 Basics of Nonlinear Modeling . . . . . . . . . . . . . . . . 64

4.1.1 The Volterra Series Model . . . . . . . . . . . . . . 64

4.1.1.1 Memory of the ROF link . . . . . . . . . 65

4.1 .2 Discrete Time Domain Issue . . . . . . . . . . . . . . . 66

4.2 Modeling the ROF Link . . . . . . . . . . . . . . . . 67

4.2.1 Photonic Band to Baseband . . . . . . . . . . . . . . 67

4.2.2 Adaptive Versus Fixed Modeling . . . . . . . . . . . . . . 67

4.2 .3 Volterra Kernel Optimization . . . . . . . . . . . . . . . . . 69

4.2.4 Filter Order and Memory . . . . . . . . . . . . . . . . . . 71

4.2.5 Simulation and Results . . . . . . . . . . . . . . 72

4.2.5.1 Modeling the Amplitude Response . . . . . . . . . 72

4.2.5.2 Modeling the Phase Response . . . . . . . . . . 73

4.2.5.3 Effect of Noise ............... . . 73

4.3 The Compensation . . . . . . . . . . . . . . . . . . 74

4.3.1 Predistortion Versus Post-Compensation . . . . . . . . . 75

4.3.1.1 The Predistortion . . . . . . . . . . . . . 75

4.3.1.2 The Post-Compensation . . . . . . . . . . . . . 76

4.3.2 A Unified Analysis of Asymmetric Compensation . . . . . . 77

4.3.3 Simulation and Results . . . . . . . . . . . . . 80

4.3.3.1 Amplitude Pre-Compensation . . . . . . . . . . . 81

4.3.3.2 Phase Pre-Compensation . . . . . . . . . . . . . 82

4.3.3.3 Amplitude Post-Compensation . . . . . . . . . . . 83

4.3.3.4 Phase Post-Compensation . . . . . . . . . . . . . 84

4.4 Concluding Remarks . . . . . . . . . . . . . . . . . 86

5 Noise and BER Analysis of A DS/CDMA Fiber Wireless System 87

5.1 The Multiuser CDMA Signal . . . . . . . . . . . . . . . . . . 88

5.2 Optical Channel Noise Mechanisms . . . . . . . . . . . . . . . . 89

5.2.1 The Shot Noise . . . . . . . . . . . . . . . . . . 89

5.2 .2 The Relative Intensity Noise . . . . . . . . . . . . . . . . 93

5.2.3 The Thermal Noise . . . . . . . . . . . . . . . . 95

5.2.4 The Dark Current Noise . . . . . . . . . . . . . . . . 95

5.3 The Signal to Noise Ratio . . . . . . . . . . . . . . . . . . . . . . . 96

5.3.1 Quantitative Discussion ................. 96

5.3 .2 Numerical Results . . . . . . . . . . . . . . . . . . . 98

5.4 Matched Filter Detection . . . . . . . . . . . . . . . . . 100 
5.4.1 Comparison of Optical and Wireless Channel Noise ..... 101

5.4 .2 The Bit Error Rate . . . . . . . . . . . . . . . . . . 102

6 A Joint Compensation to the Fiber-Wireless Channel 105

6.1 The Indoor Wireless Channel _. . . . . . . . . . . . . . . 105

6.1.1 A Tapped Delay Line Model . . . . . . . . . . . . . . . . 107

6.2 The Wiener/Hammerstein System Model . . . . . . . . . . . . 107

6.3 Nonlinear System Identification _. . . . . . . . . . . . . . . . . 109

6.3 .1 Structure Identification . . . . . . . . . . . . . . . . . 109

6.3 .2 Parameter Identification . . . . . . . . . . . . . . . . . 109

6.4 Fiber-Wireless Channel Estimation . . . . . . . . . . . . . . . . . 110

6.4 .1 Baseband/Passband Issue . . . . . . . . . . . . . . . . . 111

6.4.2 PN Sequence Correlation Method . . . . . . . . . . . . . 112

6.5 Mathematics of Channel Estimation . . . . . . . . . . . . . . . 113

6.5.1 Estimation of the Linear Part . . . . . . . . . . . . . . . 115

6.5.1.1 Multilevel Transmission . . . . . . . . . . 116

6.5.2 Estimation of the Nonlinear Part . . . . . . . . . . . . . . 119

6.5.2.1 The Least Squares Polynomial Fit . . . . . . . . 119

6.5.2.2 QR Decomposition Method . . . . . . . . . . . 120

6.6 Simulation Results . . . . . . . . . . . . . . . . . . . . 122

6.6.1 Linear System Identification . . . . . . . . . . . . . 122

6.6 .2 Nonlinear System Identification . . . . . . . . . . . . 125

7 Equalization of the Concatenated Fiber-Wireless Channel 126

7.1 An Overview on Nonlinear Equalizers . . . . . . . . . . . . 126

7.1.1 Nonlinear Equalizers without a Decision Feedback . . . . . . 128

7.1 .2 The Basic Decision Feedback Equalizer . . . . . . . . . . . 129

7.1.3 Nonlinearly Enhanced Decision Feedback Equalizers . . . . . 130

7.1.4 Proposal of a Hammerstein Type DFE (HDFE) . . . . . . . 131

7.2 Optimization of Polynomial Filter Parameters . . . . . . . . . . . . 134

7.2.1 PLF Parameters using the Channel Polynomial . . . . . . . 135

7.2.1.1 Least Squares Fit . . . . . . . . . . . . . . . . 135

7.2 .2 Direct Generation of the Inverse Polynomial . . . . . . . . 136

7.2.2.1 QR Decomposition Method ............ 136

7.2 .2 .2 Adaptive Methods .................. 138

7.3 Optimization of Linear Filter Parameters . . . . . . . . . . . . . . 139

7.3.1 Model Description . . . . . . . . . . . . . . . . . . . 140

7.3 .2 Parameter Optimization _. . . . . . . . . . . . . . . 142

7.4 Concluding Remarks . . . . . . . . . . . . . . . . . . . 145

8 Performance Evaluation of the Hammerstein type DFE 146

8.1 Evaluation of the Polynomial Filter . . . . . . . . . . . . . . 146

8.1.1 Nonlinear Transformation of Time Dispersion . . . . . . . 147 
8.1.2 Inverse Polynomial Transformation . . . . . . . . . . 150

8.1.3 Expected Value of the Polynomial Filter Error . . . . . . . . 152

8.2 Evaluation of Linear Filters . . . . . . . . . . . . . . . . . 156

8.2.1 Infinite Length Results . . . . . . . . . . . . . . . . 156

8.2 .2 Finite Length Results . . . . . . . . . . . . . . . . . 157

8.3 Numerical Results . . . . . . . . . . . . . . . . . . . . . . . . . . . . 157

8.3.1 Mean Squared Error of the Polynomial Filter . . . . . . . . 157

8.3.1.1 Real Channel Case . . . . . . . . . . . . . . . 161

8.3.1.2 Worst Case Channel . . . . . . . . . . . . . 164

8.3.2 BER Performance of the HDFE . . . . . . . . . . . 166

8.3.3 Mean Squared Error of Linear Filters . . . . . . . . . . . . . 166

8.4 Concluding Remarks . . . . . . . . . . . . . . . . 168

9 Conclusions and Suggestions for Future Work 169

9.1 Major Conclusions . . . . . . . . . . . . . . . . . . . . . . . . 170

9.1.1 Characterization and Compensation . . . . . . . . . . . 170

9.1.2 Noise and BER Analysis . . . . . . . . . . . . . . . . . . 171

9.1.3 Estimation of the Fiber-Wireless Channel . . . . . . . . 172

9.1.4 A Hammerstein type Decision Feedback

Equalizer for the Uplink . . . . . . . . . . . . . . . . . 172

9.1.5 Separate Predistortion and Equalization for the

Downlink . . . . . . . . . . . . . . . 173

9.2 Future Directions . . . . . . . . . . . . . . . . . . . . . . . . . 174

9.2.1 Compensation Related Issues . . . . . . . . . . . . . . 174

9.2.1.1 Memory of the Nonlinear System . . . . . . . . . 175

9.2.1.2 Training the Predistortion Filter . . . . . . . . 176

9.2 .2 Additional Issues of the Optical Link . . . . . . . . . . . . 176

9.2.2.1 Laser Chirp . . . . . . . . . . . . . . . . 177

9.2.2.2 Clipping Distortion . . . . . . . . . . . . . 177

9.2 .3 System Related Issues . . . . . . . . . . . . . . . . 177

9.2.3.1 Multiple PN Sequence Issue . . . . . . . . . . . 178

9.2.3.2 Multiple Cell Issue . . . . . . . . . . . . . . . 178

$\begin{array}{ll}\text { References } & 187\end{array}$

$\begin{array}{ll}\text { Appendix } & 188\end{array}$

A Baseband Representation of a Passband Nonlinearity 188

A.1 Bandpass Nonlinear Distortion . . . . . . . . . . . . . . . . . 188

A.2 Inband Nonlinear System . . . . . . . . . . . . . . . . . . . 189 


\section{List of Tables}

2.1 Parameters of the laser rate equations . . . . . . . . . . . . 26

3.1 Key specifications of the optical transmitter PFT $1700 \ldots \ldots$

3.2 Key specifications of the optical receiver PFR $1701 \ldots \ldots$

4.1 A summary of asymmetric memoryless compensation schemes . . . 85 


\section{List of Figures}

1.1 Proposed fiber based wireless access scheme . . . . . . . . . 4

1.2 Double side band spectrum resulting from direct modulation of a laser diode . . . . . . . . . . . . . . . . 6

1.3 A closer look at the fiber-wireless downlink . . . . . . . . . . 8

1.4 The RF-optical external modulation scheme . . . . . . . . . . . 9

1.5 The RF-optical direct modulation scheme . . . . . . . . . . . . 10

2.1 Basic parameters for characterizing a nonlinearity . . . . . . . 23

2.2 Electrical to optical conversion curve in a typical semiconductor laser 24

2.3 Sideband cancellation effect due to chromatic dispersion . . . . . 34

2.4 Simulation model to identify the nonlinear transfer function of the laser diode . . . . . . . . . . . . . . . . . . . 39

2.5 Simulation model of the ROF link plus AWGN wireless channel . . 39

2.6 Output optical power from the laser as well as the electrical power from the detector . . . . . . . . . . . . . . . . 40

2.7 Output QPSK constellation with of a single user . . . . . . . . 41

2.8 Output QPSK constellation with two users . . . . . . . . . . 42

3.1 A single tone at the input and output of the ROF Link . . . . . 46

3.2 Amplitude response of the ROF link with two different input powers 47

3.3 Phase response of the ROF link with two different input powers . . 47

3.4 AM-AM and AM-PM characteristics of the ROF link . . . . . . 48

3.5 Two-tone test results of the ROF link . . . . . . . . . . . . . . . 49

3.6 Noise floor measurements of the ROF link . . . . . . . . . . . . 50

3.7 Frequency dependent noise floor of the ROF link . . . . . . . . . 50

3.8 Experimental setup for dynamic measurements . . . . . . . . . . 53

3.9 Amplitude and phase distortions of vector modulated symbols after transmission through the ROF link ............. 54

3.10 Effect of the interference power when multiuser signals are transmitted through the ROF link . . . . . . . . . . . . 57

3.11 Effect of the interfering frequency when multiuser signals are transmitted through the ROF link . . . . . . . . . . . .

3.12 Power spectral density of a spread spectrum signal after transmission via the $\mathrm{ROF}$ link at $-10 \mathrm{dBm} \ldots \ldots \ldots . \ldots . \ldots 58$ 
3.13 Power spectral density of a spread spectrum signal after transmission

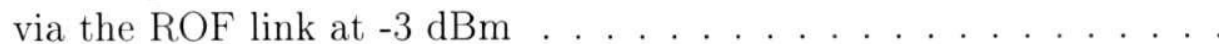

3.14 Power spectral density of a spread spectrum signal after transmission

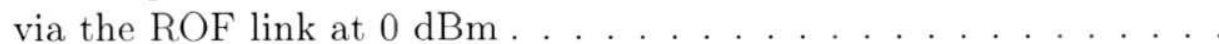

3.15 Power spectral density of a spread spectrum signal after transmission via the $\mathrm{ROF}$ link at $+3 \mathrm{dBm} \ldots \ldots . \ldots . . . \ldots$

4.1 Adaptive modeling arrangement of the ROF link . . . . . . . . . . 68

4.2 Detailed arrangement of the adaptive modeling scheme . . . . . . . 68

4.3 Variation of minimum mean squared error (MMSE) with filter order 70

4.4 Tracking the measured data with different filter orders . . . . . . . 71

4.5 Asymmetric compensation arrangement of the fiber-wireless access scheme . . . . . . . . . . . . . . . . . 75

4.6 Training arrangement for the predistortion filters . . . . . . . . . . 77

4.7 Training arrangement for the post-compensation filters . . . . . . . 77

4.8 Effect of the dynamic range on compensation . . . . . . . . . . . 81

4.9 Amplitude pre-compensation using a third order filter . . . . . . . . 82

4.10 Amplitude post-compensation with a second order filter . . . . . . . 83

4.11 Phase post-compensation using a third order filter . . . . . . . . . . 84

4.12 The final asymmetric compensation arrangement . . . . . . . . . 85

5.1 Block diagram showing a fiber based wireless CDMA system . . . . 88

5.2 Block diagram showing detected and filtered currents . . . . . . . . 89

5.3 Down converted input spectrum and the first and second order frequency response of the receiver filter . . . . . . . . . . . . . . . 91

5.4 Various noise powers of the ROF link as a function of input RF power 98

5.5 Signal to noise ratio due the relative intensity noise only . . . . . . 99

5.6 Total signal to noise ratio of the ROF link . . . . . . . . . . . . . 99

5.7 BER curves of a fiber wireless CDMA system with matched filter detection in the noise limited region. . . . . . . . . . . . . . 103

6.1 Block diagram of a Wiener system . . . . . . . . . . . . . . . . . 107

6.2 Block diagram of a Hammerstein system . . . . . . . . . . . . . . 108

6.3 Block diagram of the fiber-wireless Uplink . . . . . . . . . . . . . . 112

6.4 Reduced block diagram of the fiber-wireless uplink . . . . . . . . . . 113

6.5 Block diagram of the fiber-wireless uplink showing separable Volterra kernels . . . . . . . . . . . . . . . . . 113

6.6 Block diagram of the simulation model in Simulink ${ }^{T M} \ldots \ldots$. . . . 122

6.7 Actual and estimated wireless channel impulse responses with four discrete paths . . . . . . . . . . . . . . . . . 123

6.8 Actual and estimated wireless channel impulse responses with eight discrete paths . . . . . . . . . . . . . . . . . 123

6.9 Received data and the estimated polynomial for two different cases of the nonlinear channel . . . . . . . . . . . . . . . . . 124 
7.1 The basic decision feedback equalizer . . . . . . . . . . . . . . . 129

7.2 A general block diagram of some previous nonlinearly enhanced decision feedback equalizers (NEDFE) . . . . . . . . . . . . . . 130

7.3 Proposed Hammerstein type decision feedback equalizer . . . . . . . . 131

7.4 Complete block diagram of the fiber-wireless channel and the receiver 133

7.5 The polynomial filter and linear filters operate in two different domains 133

7.6 Decision feedback part of the proposed HDFE receiver . . . . . . . 140

8.1 Typical impulse response of a wireless channel . . . . . . . . . . . 147

8.2 Inter symbol interference of a nonlinear dispersive channel . . . . . 149

8.3 Number of interfering symbols after a double polynomial transforma-

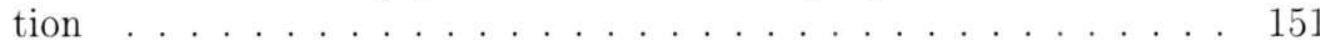

8.4 Measured impulse response at an indoor environment, courtesy [1] . 158

8.5 Higher order expectations of a channel that has impulse response shown in Fig. 8.4 . . . . . . . . . . . . . . . . . . . . . . . . . . . . . 158

8.6 MSE of the polynomial filter versus number of bins $L_{b}$ for a third order compensation . . . . . . . . . . . . . . . . . . 159

8.7 MSE of the polynomial filter versus number of bins $L_{b}$ for a second order compensation . . . . . . . . . . . . . . . 159

8.8 MSE of the polynomial filter versus SNR for a second order compensation ........................... 160

8.9 MSE of the polynomial filter versus SNR for a third order compensation 160

8.10 Variation of the parameter $\sigma_{h}$ with channel memory for the worst case channel . . . . . . . . . . . . . . . . . . . . . . 162

8.11 Mean squared error of the polynomial filter as a function of Channel Memory with a third order compensation for the worst case channel 162

8.12 Mean squared error of the polynomial filter as a function of Channel Memory with a second order compensation for the worst case channel 163

8.13 Mean squared error of the polynomial filter as a function of signal to noise ratio with a third order compensation for the worst case channel 163

8.14 Mean squared error of the polynomial filter as a function of signal to noise ratio with a second order compensation for the worst case channel ......................... 164

8.15 MMSE as a function of the feed forward filter delay . . . . . . . . . 165

8.16 Weights of the feedback filter that is compared to the channel postcursors . . . . . . . . . . 167

8.17 Bit error rate performance of the HDFE and a standard DFE . . . 167

9.1 Downlink compensation arrangement . . . . . . . . . . . . . . . 174

9.2 Different ways of obtaining feedback signal to the predistortion filter 175

A.1 Inphase and Quadrature Representation of a Nonlinear System . . . 189 


\section{List of Symbols}

Some Conventions
$\hat{x}, x_{e s t}$
$x x(n)$
$E[x],\langle x\rangle, \bar{x}$
$\sigma_{x}^{2}$
$\operatorname{Var}[x]$
$\sigma_{x}^{i}$
$J_{x}$
$\Re_{i j}$
$\boldsymbol{V}_{x}$
$\boldsymbol{Q}_{x}$
$\boldsymbol{R}_{x}$
$\boldsymbol{A}^{T}$
$\widetilde{W}$
$X^{o p}$

Greek Symbols

$\alpha$

$\alpha_{i}$

$\beta$

$\Gamma$

$\Delta$

$\Delta_{\text {opt }}$

$\Delta P$

$\delta(t)$

$\epsilon$

$\epsilon_{l}$

$\epsilon_{p}$

$\eta$

$\eta_{q}$

\section{Description}

An estimate of random variable $x$

A zero mean process derived from $x(n)$

Expectation of variable $x$

Variance of the variable $x$

Variance of the variable $x$

$i^{t h}$ order expectation of variable $x$

Mean square value of variable $x$ or, $E\left[x^{2}\right]$

Correlation between variables $i$ and $j$

Vondermonde matrix of variable $x$

The orthonormal unitary matrix resulting from

QR decomposition of $\boldsymbol{V}_{\boldsymbol{x}}$

The upper triangular matrix resulting from

QR decomposition of $\boldsymbol{V}_{\boldsymbol{x}}$

Transpose of matrix $\boldsymbol{A}$

Augmented version of vector $\boldsymbol{W}$

Optimal value of $\mathrm{X}$

\section{Description}

Dispersion coefficient of a single mode fiber

$i^{\text {th }}$ amplitude of the PN sequence $x(n)$

Fraction of the spontaneous emission coupled into laser mode

Optical confinement factor in the laser rate equation

Delay of the feed forward filter

Optimum delay of the feed forward filter

Instantaneous optical noise power

Dirac impulse function

An arbitrary small number

The linear filter error sequence

Error sequence of the polynomial filter

Interference term of the decision statistics

Noise term in the decision statistics

Quantum efficiency of the photo detector 
Phase as described in the text appropriately

Operating optical wavelength

Forgetting factor of the RLS algorithm

Source operating wavelength

Zero dispersion wavelength

Combined noise and interference term

The rms delay spread

Time delay

Electron lifetime in the laser rate equation

Photon lifetime in the laser rate equation

Phase of the input symbols

Input phase associated with the $k^{\text {th }}$ user

Responsivity of the photodetector

Gain compression coefficient of the active region

Radian carrier frequency

\section{English Symbols}

\section{Description}

$A_{i}(1 \leq i \leq l)$
$a_{k}(t)$
$a(n)$
$B$
$B_{c}$
$b_{1,1}$
$b_{k}(t)$
$c$
$D$
$d(n)$
$d_{i}(n)$
$d_{r}(n)$
$d(\lambda)$
$e(n)$
$e_{o}(t)$
$F($.
$f_{c}$
$f_{o}$
$\boldsymbol{G}$
$G($.
$G_{\text {link,dB }}$
$G_{m}$
$G_{\text {opt }}$

Coefficients of the (channel) polynomial

Pseudorandom binary code associated with $k^{\text {th }}$ user

Symbol amplitude

Bandwidth

Coherence bandwidth

First bit of the first user

Baseband data associated with the $k^{\text {th }}$ user

Speed of the light $\left(3 \times 10^{8} \mathrm{~m} / \mathrm{s}\right)$

A diagonal matrix

Desired response of an adaptive system

Intermediate symbol after predistortion in the downlink

Received symbol before post compensation in the uplink

Chromatic dispersion of a single mode fiber

Error at time $n$

Optical field output at the laser

Nonlinear mapping function of the channel

Radio carrier frequency

Optical carrier frequency

Weight vector of the polynomial filter

Nonlinear mapping function of the compensating filter

Optical link gain in $\mathrm{dB}$

Modulation gain of the laser diode

Optical receiver amplifier gain 


$G_{w l}$
$g_{i}(1 \leq i \leq N)$
$g_{i}\left(m_{1}, m_{2} \ldots m_{i}\right)$
$g_{o}$
$H$
$H_{b p}(f)$
$H_{S M F}(f)$
$H_{D}(f)$
$h(n)$
$h_{D}(t)$
$h_{p}$
$h_{p}(\ldots)$
$h_{r x}(n)$
$h_{t x}(n)$
$h_{w l}(n)$
$I_{1}$
$I_{2}(t)$
$I_{A}$
$I_{D}(t)$
$I_{D}$
$I_{d a r k}$
$I_{k}$
$I_{R I N}$
$I_{t h}$
$J(n)$
$K$
$K$
$K_{c l}$
$k_{B}$
$L$
$L_{b}$
$L_{f}$
$L_{o p t}$
$L_{w l}$
$l$
$l_{f}$
$m$
$N$
$N_{k}$
$N_{L}$
$N_{c}$
$N_{d}$
$N_{e}$

Wireless receiver amplifier gain

Weights of the polynomial filter

Separable Volterra kernel, $i^{\text {th }}$ order

Gain slope constant in the laser rate equation

Impulse response matrix of the wireless channel

The bandpass transfer function

Transfer function of a single mode fiber

Transfer function of the photodetector

Impulse Response of the linear part

Impulse response of the photodetector

Planck's constant $\left(6.626 \times 10^{-34}\right)$

The $p^{\text {th }}$ order Volterra kernel

Impulse response of the receive filter

Impulse response of the transmit filter

Impulse Response of the wireless channel

Desired user contribution to decision statistics

Detector current after the bandpass filter

Current injected into the active layer in a laser

Instantaneous detector current

Mean detector current

Dark current

$k^{\text {th }}$ user contribution to decision statistics

Relative intensity noise current

Thermal noise current

Cost function of the RLS algorithm

Total number of CDMA channels in a SS signal

Order of the modeling filter

Attenuation factor due to laser clipping

Boltzmann's constant (1.38054 X 10-23)

Memory of the wireless channel

Number of post-cursors

Number of pre-cursors

Optical channel loss

Wireless channel loss

Order of the channel polynomial

Length of the fiber

Optical modulation index

Order of the polynomial filter

Number of sample points in least squares estimation

Number of sample points

Length of the spreading (PN) code in a CDMA system

Length of the input vector $\boldsymbol{U}_{d}$

Electron density in the laser rate equations 


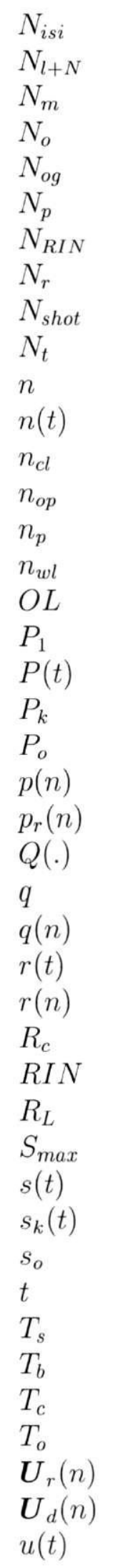

Number of interfering terms

Number of interfering terms after double transform

Memory of a Volterra system

Two sided power spectral density of the combined noise

Electron density at which the net gain zero

Photon density in the laser rate equations

Double sided RIN noise power spectral density

Length of the input vector $\boldsymbol{U}_{r}$

Double sided shot noise power spectral density

Number of levels during PN sequence training

Discrete time instance

A general noise term

Clipping noise

Optical channel noise power

Noise plus the PLF error

Wireless channel noise power

Optical link losses

Power of the first user in a CDMA system

Instantaneous optical power emitted from the laser

RF power associated with the $k^{\text {th }}$ user

Mean optical power emitted from the laser

Power of symbol $a(n)$

Power of symbol $a_{r}(n)$

The Q function

Charge of an electron (1.602 X 10-19)

Intermediate signal after the linear channel

Output RF signal from a wireless channel

Received signal from a Wiener system

Chip rate in a CDMA environment (reciprocal of $T_{c}$ )

Relative intensity noise of an optical system $(\mathrm{dB} / \mathrm{Hz})$

Load Resistance

Maximum value of the SS signal $s(t)$

The spread spectrum electrical signal

The CDMA signal associated with the $k^{\text {th }}$ user

Zero dispersion slope of a single mode fiber

Continuous time

Sampling time interval

Bit period

Chip period with direct spreading (reciprocal of $R_{c}$ )

Absolute temperature

Input vector to the post-compensation filter

Input vector to the predistortion filter

Output RF signal from the optical channel 


$\boldsymbol{U}_{a}(n)$
$\boldsymbol{U}_{p}(n)$
$\boldsymbol{u}(n)$
$V_{\text {act }}$
$v(n)$
$\boldsymbol{W}(n)$
$\boldsymbol{W}_{a}(n)$
$\boldsymbol{W}_{\boldsymbol{b}}$
$\boldsymbol{W}_{\boldsymbol{f}}$
$\boldsymbol{W}_{\phi}(n)$
$w(n)$
$w_{i}(n)(1 \leq i \leq l)$
$x(n)$
$Z_{1}$
$Z_{\text {out }}$
$Z_{\text {in }}$
$z(n)$

Input to the amplitude modeling filter Input to the phase modeling filter Input vector (to a nonlinear system) Volume of the active layer in a laser Observation noise

Weight vector

Weight vector of the amplitude filter

Weight vector of feedback filter in the DFE

Weight vector of feed forward filter the DFE

Weight vector of the phase filter

Tap weight at time $n$

Higher order terms at the output of a Wiener system Transmitted PN Sequence

Decision statistics of the first user in a CDMA system RF impedance at the optical link output

RF impedance at the input of the optical link

Output of the polynomial filter in HDFE 


\section{List of Abbreviations}

$\begin{array}{ll}\text { Abbreviations } & \text { Description } \\ \text { AF } & \text { Additional Filter } \\ \text { AGC } & \text { Automatic gain controller } \\ \text { AM-AM } & \text { Amplitude dependent amplitude distortion } \\ \text { AM-PM } & \text { Amplitude dependent phase distortion } \\ \text { APD } & \text { Avalanche photo diode } \\ \text { ARMA } & \text { Auto regressive moving average } \\ \text { AWGN } & \text { Additive white Gaussian noise } \\ \text { BER } & \text { Bit error rate } \\ \text { BIBO } & \text { Bounded input bounded output } \\ \text { BPSK } & \text { Binary phase shift keying } \\ \text { BS } & \text { Base station } \\ \text { CATV } & \text { Cable Television } \\ \text { CDMA } & \text { Code division multiple access } \\ \text { CO } & \text { Central office } \\ \text { CW } & \text { Continuous wave } \\ \text { CWTA } & \text { Canadian wireless and telecommunications association } \\ \text { DD } & \text { Direct detection } \\ \text { DFE } & \text { Decision feedback equalizer } \\ \text { DR } & \text { Dynamic range } \\ \text { DS } & \text { Direct sequence (also direct spreading) } \\ \text { DSB } & \text { Double side band } \\ \text { DSP } & \text { Digital signal processing } \\ \text { dB } & \text { Decibels } \\ \text { E/O } & \text { Electrical to optical (conversion) } \\ \text { EDFE } & \text { Enhanced decision feedback equalization } \\ \text { EIN } & \text { Equivalent input noise } \\ \text { EOM } & \text { External optical modulator } \\ \text { FBF } & \text { Feedback filter } \\ \text { FFF } & \text { Feed forward filter } \\ \text { FCC } & \text { Federal communications commission } \\ \text { FDMA } & \text { frequency division multiple access } \\ \text { FM } & \text { Frequency modulation } \\ \text { GSM } & \text { Global system for mobile communications } \\ & \end{array}$




\begin{tabular}{|c|c|}
\hline Gbps & Giga bits per second \\
\hline HDFE & Hammerstien type decision feedback equalizer \\
\hline HPA & High power amplifier \\
\hline IIP3 & Input intercept of third order \\
\hline IM & Intensity modulation \\
\hline IN & Interferometric noise \\
\hline IRIS & Impulse response instrumentation system \\
\hline ISI & Inter symbol interference \\
\hline $\mathrm{kb} / \mathrm{s}$ & Kilo bits per second \\
\hline LAN & Local area network \\
\hline LMCS & Local multipoint communication services \\
\hline LMS & Least mean squares \\
\hline LOS & Line of sight \\
\hline LTV & Linear time variant \\
\hline MAI & Multiple access interference \\
\hline $\mathrm{Mb} / \mathrm{s}$ & Mega bits per second \\
\hline MDP & Minimum detectable power \\
\hline MPSK & M-ary phase shift keying \\
\hline MSE & Mean squared error \\
\hline MMSE & Minimum mean squared error \\
\hline MT & Mobile terminal \\
\hline MZI & Mach zehnder interferometer \\
\hline NARMAX & Nonlinear ARMA model with exogenous inputs \\
\hline NEDFE & Nonlinearly enhanced decision feedback equalizers \\
\hline NLD & Nonlinear distortion \\
\hline NLTI & Non linear time invariant \\
\hline $\mathrm{O} / \mathrm{E}$ & Optical to electrical (conversion) \\
\hline OMD & Optical modulation depth \\
\hline PBX & Private branch exchanges \\
\hline PCS & Personal communication services \\
\hline PIN & P-type - intrinsic - n-type (photo detector) \\
\hline PLF & Polynomial filter \\
\hline PN & Pseudo noise \\
\hline PMD & Polarization mode dispersion \\
\hline PSD & Power spectral density \\
\hline PSK & Phase shift keying \\
\hline QoS & Quality of service \\
\hline QPSK & Quadrature phase shift keying \\
\hline RAP & Radio access point \\
\hline $\mathrm{RF}$ & Radio frequency \\
\hline RIN & Relative intensity noise \\
\hline RLS & Recursive least squares \\
\hline ROF & Radio over fiber \\
\hline
\end{tabular}


rms

SFDR

SINR

SIR

SM

SNR

SS

TDMA

TOI

$\mathrm{UHF}$

VSWR

WH
Root mean square

Spurious free dynamic range

Signal to interference and noise ratio

Signal to interference ratio

Single mode

Signal to noise ratio

Spread spectrum

Time division multiple access

Third order intercept

Ultra high frequency

Voltage standing wave ratio

Wiener/Hammerstein system 


\section{Chapter 1}

\section{Motivation and Background}

\subsection{The Evolution of Wireless Communications}

The future of wireless will not just be cell phones; it will be an essential part of our daily life. Wireless devices, either embedded in other systems or stand-alone, will be used everywhere. This will range from market places to space stations; from luggage tags to missile controllers, yet be transparent much like the air itself. Surveys have shown that a new wireless subscriber signs up every 2.5 seconds. According to the IEEE Communications Magazine, over 100 billion dollars will be invested in the coming years in wireless technologies and a major portion of this investment will go towards providing advanced multimedia services. Simply saying, wireless communications is the next phase in the information revolution and the next storm in the communications industry.

Considering only the voice communications, the number of cell phones increases exponentially. For example, in Japan this number goes from approximately 60 million in year 2000 to 80 million in year 2006, measuring a penetration ratio of approximately $70 \%$ by 2006 [2]. The number of cellular and personal communications system (PCS) users in the US has surpassed 100 million. The number of wireless telephones in Canada increases by 20-30\% every year (Canadian Wireless Telecommunications Association). The trend is similar worldwide.

Wireless is also at the edge of a significant revolution - broadband multidimensional wireless emerges in all areas of our information society. Convergence of the wireless and the Internet has dramatically increased the demand. Wireless 
e-business penetrates everywhere. Corporate sites expand their e-business services spending millions of dollar everyday. More users require simultaneous multidimensional high data-rate access than ever. The number of wireless internet subscribers exploded from zero to 15 million in just 17 months in Japan only [2]. Wireless WebTV with multi-casting capabilities is on the rise. All these show that the "second wave" in wireless communications has arrived. In this scenario, more resources will be occupied by multimedia services such as real time video and data than by voice. Data size will continue to increase year by year and the bit-rate capability of the wireless systems must follow it. Furthermore, reliability and the quality of service is much more essential for wireless data services than for voice services.

\subsubsection{The Bandwidth Quest}

The ever increasing crave for bandwidth is due to two factors: first is the increment in the number of users; second is the excessive bandwidth requirement of each user to support advanced services. This bandwidth quest postulates significant pressure on wireless system planners because of the limited radio spectrum. Hence, a myriad of techniques is being researched to increase the system capacity.

Obviously, the most widely used approach is increasing the frequency reuse factor. Frequency reuse factor defines the number of radio cells that will use a set of frequency channels. The efforts in this category range from simple cell splitting and sectoring to advanced techniques such as space division multiple access with smart-antennae. The second category attempts to increase the system capacity without increasing the frequency reuse. These approaches range from coding and error correction schemes to advanced modulation techniques. However, Shannon's theorem limits the capacity per unit bandwidth and only marginal improvement is possible with these techniques. Finally, as the lower end of the spectrum is crowded there are ongoing efforts to use the unused high frequency spectrum, which goes up to millimeter wave bands. A good example is the local multipoint communications services (LMCS) standard that allocates $1 \mathrm{GHz}$ per service provider at $28 \mathrm{GHz}$ band for CATV distribution. Even the $60 \mathrm{GHz}$ band is recently allocated by the FCC for wireless access. However, at these ultra high frequencies, coverage area for a given transmitted power decreases drastically. This is because of the fact that, 
the free-space attenuation of an electromagnetic wave is proportional to the square of its frequency [3]. Therefore, cells should be much smaller at these ultra high frequencies.

\subsection{Indoor Micro/Pico Cells}

From the discussion in Section 1.1, it is obvious that micro and pico cells will play a major role in wireless communications soon. This is especially the case in indoor applications because, multimedia services are mostly used in indoors and the user penetration is high in indoor environments. For example, wireless data communication (LAN type) services and cordless PBX systems are in high demand in indoor offices.

The microcellular architecture would not only give better frequency reuse and coverage, but also would reduce power consumption and size of the portable units. With this microcellular arrangement, the wireless link is short, so that the rms delay spread is small. This small delay spread results in a low inter symbol interference for a given bit rate. In addition, in microcells, often a strong line of sight path is available between the transmitter and the receiver. As a result, high-speed multimedia services are more easily accommodated in indoor microcells than outdoor macrocells. Furthermore, crowded places like campus premises and downtown offices can be cost-effectively configured into microcells. This is also useful in hidden areas like tunnel zones where macro-cellular wireless signals would not typically reach.

Finally, in future wireless systems, the users will be categorized according to their mobility and concentration. For example, in IMT2000 (International Mobile Telecommunications 2000), indoor (pedestrian) users are served with 2 Mbps while, high mobility users are allowed only 144 kbps. From the foregoing discussion, it is obvious that the cellular architecture will be heterogeneous in the near future, providing different level of subscriber services depending on the cell size. Users, who need high bandwidth will typically be in indoors and would be better served by micro/pico cells. 


\subsubsection{The Radio Access Point (RAP)}

In the micro/pico cellular scenario, the number of cells would be large and would require a large number of base stations. It would be too costly to build large number of complete base stations in each micro/pico cell. In this thesis, a more economical approach is proposed. In this proposal, a group of base stations is replaced by a single base station and a number of radio access points as shown in Fig. 1.1. For downlink transmission, data is first processed and converted to optical at the central base station for transmission over the ROF link. At the RAP, optical signal is converted to RF, which is amplified and then relayed to the portable units via wireless channels. For uplink reverse operations are performed.

Since the number of RAPs will be large, they should be typically cost effective, small, robust and of low complexity. To meet these requirements, number of functions that is performed by an RAP would be minimal. For example, there would be no up conversion from baseband to radio frequencies or vice versa at the RAP.

In Fig. 1.1 it is seen that, the RAP must consist of at least one O/E (optical to

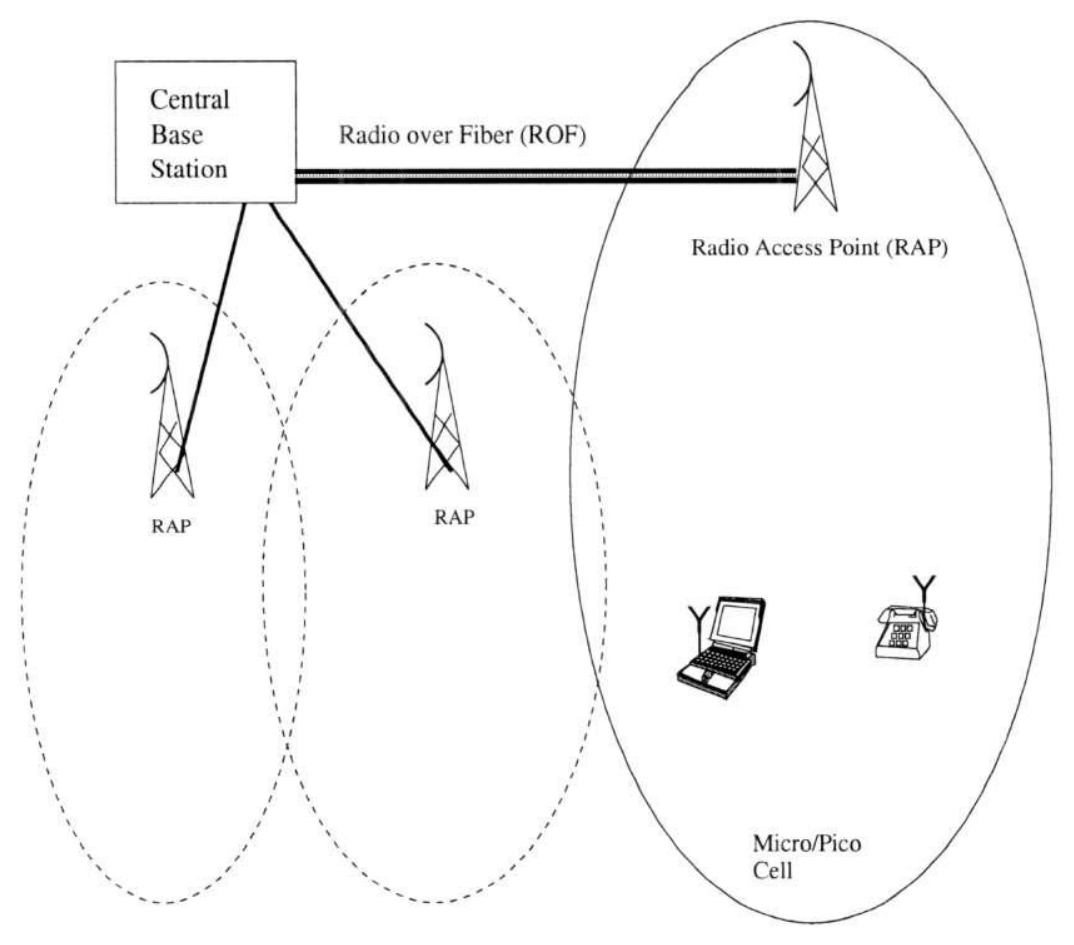

Figure 1.1: Proposed fiber based wireless access scheme 
electrical converter), E/O(electrical to optical converter), and a radio antenna. In the downlink, RF amplifiers need to be incorporated to compensate for conversion loss. Furthermore, it is attractive to make these RAPs signal format independent so that a single physical layer can support multiple services. The power consumption and maintenance requirements of the RAP's should be low. Most of the processing such as up/down conversion, modulation/demodulation, equalization and error correction should be done at the central base station or at the portable unit.

\subsubsection{Asymmetry in Complexity}

In order to keep the RAP simple, the processing complexity should be moved away from the RAP, either to the portable units or to the central base station as much as possible. However, shifting most of the signal processing to the central base station is the best solution because, the cost of the central base station is shared among all the users. Therefore, processing capability, power consumption and maintenance requirements are not a concern in the central base station. The signal processing can be easily optimized and updated in a centralized environment. Typically, the equipment in central base station sits in a controlled environment, assuming indoors. Therefore, degradations due to environmental variations are a minimum in the central base station. For the reasons outlined above, the processing at the portable units should be a minimum also.

There have been ongoing research efforts to move complexity from hand sets to the base station in conventional cellular systems. For example, there is extensive work done to reduce the complexity of the portable unit by utilizing the reciprocity of the radio channel at TRLabs, Calgary [4]. One of the objectives of this work is to explore the possibility of doing most of the signal processing at the central base station so that, not only the portable unit but also the RAP becomes less complex. However, in this case we are dealing with the wireless channel in series with the nonlinear fiber channel. Therefore finding a solution is much more challenging in this case. 


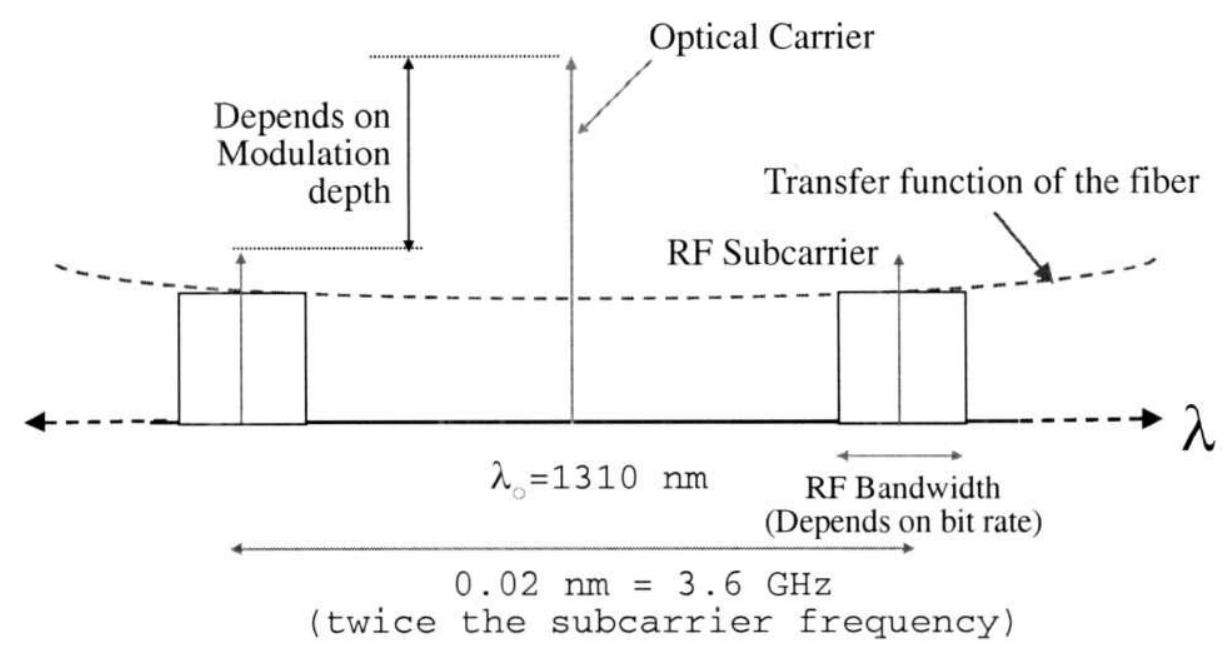

Figure 1.2: Double side band spectrum resulting from direct modulation of a laser diode

\subsection{Optical Fiber Based Wireless Access}

Connecting relatively large numbers of RAPs to the Central Office is an issue that needs to be addressed. Optical fiber is the best medium because of its abundant bandwidth and low distortion properties. The optical signal is inherently immune to electromagnetic interference. Furthermore, in contrast to co-axial cables, every additional kilometer length of fiber increases the loss by only about one dB. These factors make the fiber the most attractive solution in interconnecting these wideband pico cells.

\subsubsection{Baseband vs Subcarrier Transmission}

Traditionally, the signal is transmitted via fiber in the baseband using digital modulation techniques, by turning on and off the optical source. With this approach the optical carrier is directly modulated by the baseband information sequence. Baseband modulation with data speeds up to $10 \mathrm{Gbps}$ is commercially available. Furthermore, efforts are on the way, for example, to increase the speed up to 40 Gbps or even up to 160 Gbps.

However, when the modulating signal itself is in the radio frequency, the best way is to use an analog modulation scheme widely known as the 'subcarrier modulation'. 
Here, the RF signal is superimposed on the DC bias current, which then drives the optical source. In this thesis, we refer to this type of analog subcarrier transmission as the radio-over-fiber ( $\mathrm{ROF}$ ) transmission. This method is extensively used in analog cable TV distribution systems. In this work we will be considering only the radio-over-fiber approach.

In an ROF link, the spectrum inside the fiber consists of both the optical carrier plus the RF subcarriers, as shown in Fig. 1.2. In Fig. 1.2, energy in the sidebands depends on the modulation depth or modulation index $m^{1}$ which, is defined in equation (2.1). The RF bandwidth depends on the information bit rate.

There are several advantages with radio-over-fiber transmission. Most importantly, high-speed A/D and D/A conversion requirements are eliminated because the RF signal can be used as it is. Besides, analog optical fiber links have the ability to transmit radio waves up to tens of $\mathrm{GHz}$ without any format conversion Furthermore, when there are multiple RF carriers, they all can simply be summed and used to modulate a single optical carrier. In this way, all the RF carriers can be simultaneously transmitted via the fiber in a subcarrier multiplexed way.

In addition, microwave signals can even be optically processed. New techniques such as Fiber Bragg gratings enable RF signals to be filtered and tuned optically. These make the ROF approach more attractive and open new possibilities. For example Bob Davies et al. [5] have demonstrated an $18 \mathrm{GHz}$ carrier generation by harmonic up conversion, using the laser nonlinearity at TRLabs, Calgary. A low loss, down converting system is described in [6].

\subsubsection{A Two Stage Modulation}

There are two entirely different forms of modulation/demodulation processes involved in the radio-over-fiber scheme. This can be better understood by looking at Fig. 1.3.

1. Modulating the RF carrier with the baseband information sequence at the central base station and the corresponding demodulation at the portable.

${ }^{1}$ In this thesis, both of these terms are used with the same meaning. 


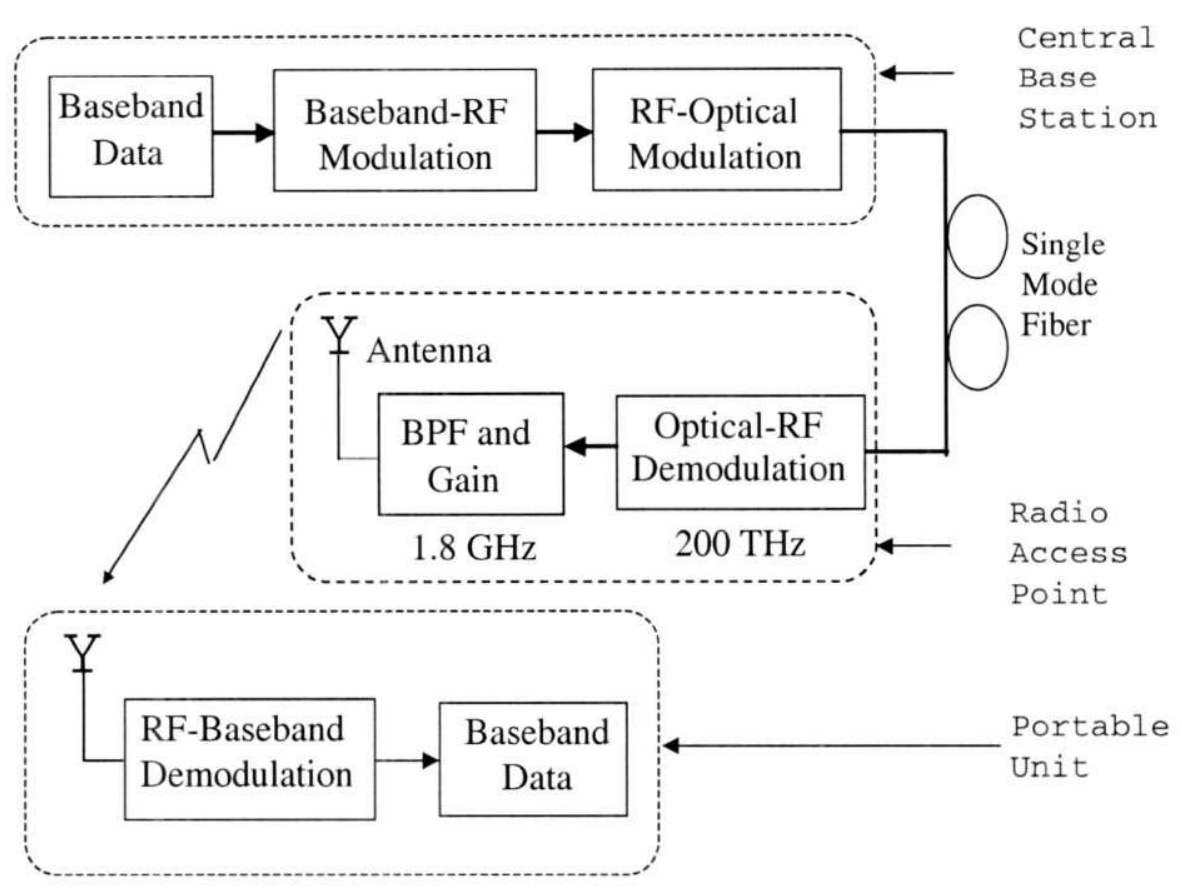

Figure 1.3: A closer look at the fiber-wireless downlink

2. Modulating the optical carrier with the RF signal at the laser and the corresponding demodulation at the optical receiver.

To avoid confusion, the first modulation will be referred to as the baseband-RF modulation and the second modulation will be referred to as the RF-optical modulation in this thesis.

\subsubsection{External and Direct Modulation}

The RF-optical modulation is done either by directly modulating the optical source itself (direct modulation) or by modulating the optical carrier after generation (external modulation). There are benefits and drawbacks in each of these approaches.

\section{External Modulation}

Here, the laser is constantly driven with a direct bias current and the optical output is either phase or intensity modulated by an external optical modulator (EOM), as shown in Fig. 1.4. 


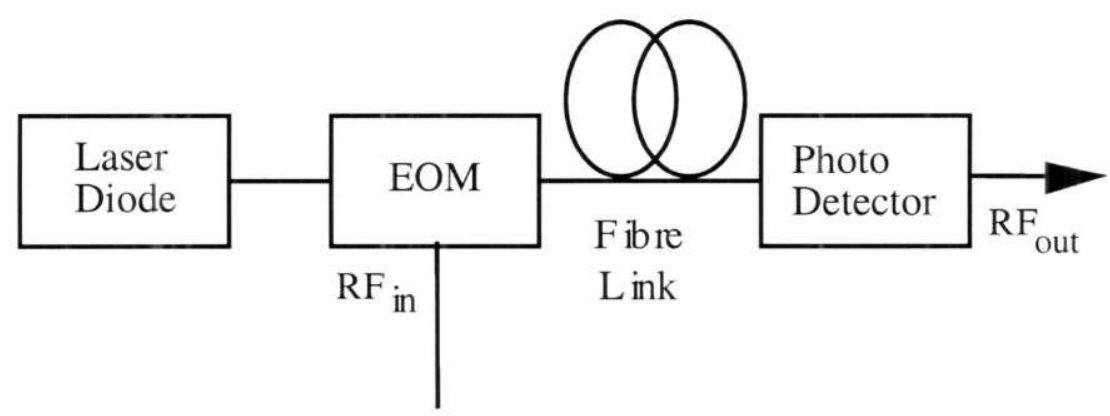

Figure 1.4: The RF-optical external modulation scheme

Mach-Zehnder Interferometer (MZI): The most widely used external modulator is the Mach-Zehnder Interferometer (MZI). These modulators provide very high bandwidth (up to $40 \mathrm{GHz}$ ) and are capable of handling at least 400 $\mathrm{mW}$ of continuous optical power [6] and the gain of the externally modulated link is proportional to the square of optical power. So very high gain links are achievable with high bandwidth. However, the nonlinearity starts to appear at high power levels. The MZI are expensive and require precise configuration.

Electro Absorption Modulator (EAM): This is another type of external modulator that is emerging in this field. Due to its multi quantum well structure, the EAM can also be optimized to detect optical signals. This is very attractive for full duplex mode operation. These EAMs are highly desirable to be used as single unit RAPs because, these are small, robust and more importantly enables a passive operation. Therefore, they can be used as both $\mathrm{O} / \mathrm{E}$ and E/O converters, even without an external power supply. The EAM was the first device that induced our attraction for research in this area. However, due to unavailability of this device for this research work, it has been decided to stick to commercially available devices.

\section{Direct Modulation}

With this approach, the driving current of the optical source (laser) is directly modulated by radio frequency signal. This method is robust, cost effective and gives adequate performance when the radio frequency is a few $\mathrm{GHz}$. The maximum 


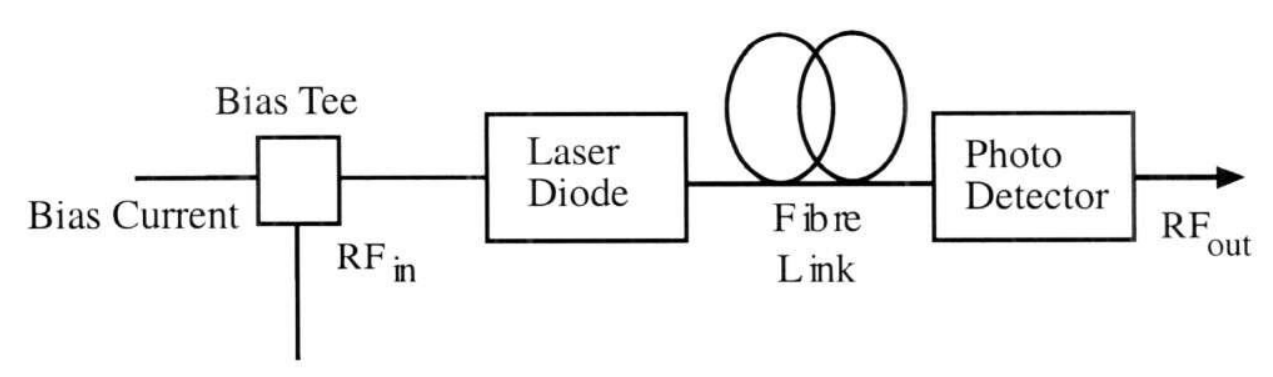

Figure 1.5: The RF-optical direct modulation scheme

bandwidth of a direct modulated link is about $20 \mathrm{GHz}$ [7]. However, a directly modulated link severely attenuates the RF signal. The loss is about $39 \mathrm{~dB}$ (without the fiber loss) for a resistively matched ROF link and about $20 \mathrm{~dB}$ for a reactively matched ROF link [8]. Furthermore, directly modulated lasers exhibit chirp and relative intensity noise, which are discussed later in this thesis. In this work our focus is limited to direct modulation.

\subsubsection{Major Limitations}

The ROF link has enough bandwidth to support many wideband wireless channels. When the radio frequency is only a few $\mathrm{GHz}$, and the fiber length is only a few kilometers, fiber dispersion or laser chirp is not a concern. However, when a wireless link is in series with the optical link, the nonlinear distortion (NLD) in the ROF link is the biggest concern. Laser diode's intrinsic nonlinearity is considered as the major source of this NLD in a directly modulated link. This is severe in the uplink because of the multipath fading of the wireless channel. Even in the downlink, a good dynamic range is required because, the number of active users keeps varying. For example in IS-95, the number of downlink channels can vary from 9 to 64 .

The dynamic range requirement is more severe than we would imagine. In a typical micro cellular environment, the distance $(d)$ from a portable to the radio access point can vary from few meters (the closest user), to about 300 meters (the most remote user). In addition, the average path loss of the wireless link can vary from $d^{-1.5}$ to $d^{-4}$ depending on the obstacles in between. Thus, in indoor picocells the radio signals can fluctuate by $40-55 \mathrm{~dB}$ and in outdoor microcells 
this fluctuation can be as high as $80-90 \mathrm{~dB}$ [9]. This amount can vary by up to $10 \mathrm{~dB}$ with varying environmental characteristics [10]. This dynamic range (DR) has to be added with the minimum acceptable carrier to interference $(\mathrm{C} / \mathrm{I})$ ratio of the particular modulation scheme. For example, at least a $\mathrm{C} / \mathrm{I}$ ratio of $16 \mathrm{~dB}$ is required for IS-54.

In addition, the linear range of a given device inherently goes down with an increasing bandwidth. This is aggravated by the fact that, the noise power increases with the signal bandwidth, which, in turn, decreases the DR. Hence, the wider the signal bandwidth, the more severe the nonlinear distortion. This postulates a severe constraint on the linearity requirement to support wideband multimedia services. For example, the DR requirement is much severe for wide band CDMA type systems (1.25 MHz or $5 \mathrm{MHz}$ ) than for narrow band systems.

From our measurements using the best commercially available devices as well as from the literature, we found that the dynamic range available from a typical ROF link is $20-30 \mathrm{~dB}$ less than what is needed to serve a typical microcell.

\subsection{Previous Attempts to Reduce NLD}

Since, the dynamic range requirement for a wireless link is too high to be met by either the laser diode or by an external modulator, several ways to decrease this requirement or to linearize these devices have been investigated. Those attempts are briefly reviewed in this section.

\subsubsection{Automatic Gain Controllers and Attenuators}

A widely attempted solution is the automatic gain controller. However, automatic gain controllers typically have long response time. Therefore, transient response of the automatic gain controller increases call-dropping probability during sudden power changes. This makes them not suitable for the fast changing wireless scenario This is especially the case during power on and off conditions. A slight variation, called an automatic attenuation scheme to suppress the signals within the linear range, is proposed in [11]. Here, depending on the input RF power, an attenuator turns on or off to keep the signal within the linear range. In addition, a control signal 
should be transmitted to the receiving end to inform about the level of attenuation. It will be more complicated to transmit extra information through the link and also to have two sets of variable attenuators and amplifiers at both ends.

\subsubsection{Fixed Electronic Solutions}

Equivalent circuit model based solutions for the laser diode have been developed in [12] and [13]. Tucker et al. [14] have demonstrated a $10 \mathrm{~dB}$ improvement on both the laser intensity noise and the third order inter-modulation distortion utilizing an electro/optical feed forward compensation technique Gysel et al. [15] have demonstrated a circuit that reduces the chirp in the small signal region.

Raziq et al [16] have reported an analysis and a compensation scheme for the nonlinearity using a post-compensation block. They have analytically derived a third order Volterra kernel representation of the laser diode. Then a post-recovery block is included after the optical receiver so that the nonlinear distortion of the laser diode is cancelled out. From their simulation, the third order inter modulation distortion has been decreased by $16-22 \mathrm{~dB}$ in a CDMA link. The improvement depends on the code length and the number of users. The physical constants of the laser rate equation are used to calculate parameters of the recovery block.

The major drawback in these fixed circuit solutions for the laser diode is their device dependency. Their accuracy depends on knowledge of physical parameters. Considering the fact that large differences in the modulation response of similar distributed feedback lasers have been reported in [17], these solutions have to be optimized for each and every device. In addition, these solutions are fixed and unable to track any changes. Furthermore, in wireless applications, high gain receiver amplifiers introduce additional nonlinear distortions, which need to be considered.

\subsubsection{Network Layer Approaches}

Some authors have proposed to mitigate the NLD in the network layer. One way is to allocate frequency channels to the ROF link in a way such that the cross modulation products do not overlap. W. I. Way [9] says that, re-programming the channel synthesizer at the base site can change this assignment dynamically and, 
the ability to continuously monitor and control the carrier frequencies with this dynamic scheme can reduce the intermodulation products. This needs a special channel assignment algorithm with additional constraints.

There is another proposal to introduce RAP diversity [18] to mitigate the nonlinear distortion. Simulation results show a good reduction in the linearity requirement of the reverse ROF link when the portable unit communicates with two or more RAP simultaneously. This actually is a form of macro-diversity.

\section{A Two Stage Cancellation Technique}

Recently, some two-stage cancellation techniques for the multiuser interference in a wireless DS/CDMA environment have been investigated. This work was extended by W. Huang et al. [19] to cancel both the multiuser interference and the nonlinear distortion together in a fiber based wireless link. Here again the optical link is approximated by a third order polynomial. Theoretical analysis and numerical results show orders of BER performance improvement with this cancellation technique.

\subsubsection{Miscellaneous Attempts}

\section{FM Subcarrier Transmission}

In an FM subcarrier transmission, the optical carrier is frequency modulated by the RF signal. If the carrier frequency is high enough, the high order harmonics would not be superimposed on the fundamental signal. Thus the FM signal is immune to laser diode nonlinearity [20]. However, the voltage controlled oscillator, mixer and the local oscillator have to be selected to eliminate the second order distortion. The drawback of this approach is the difficulty associated with the frequency modulation and demodulation of the optical carrier which need coherent generation and detection of the optical carrier.

\section{Superimposed Subcarrier Modulation}

In superimposed subcarrier modulation, an additional subcarrier $f_{o}$, usually far away from the desired center frequencies $f_{1}, f_{2} \ldots$ etc., is injected into the fiber. This process broadens the spectral width of the laser diode. This wide spectral width 
reduces the emitted light's coherency, which in turn reduces the relative intensity noise degradation [9]. These are non-standard way of spreading the signal that is described below.

\section{Spreading Approaches}

Some efforts have been reported to decrease linearity requirement by spreading the radio signal with a PN sequence just before the laser diode. These are different from the transmission of an inherently spread spectrum radio signal such as IS-95 signal via fiber.

A scheme for spreading the GSM signal in order to reduce the RF power fluctuation before optical modulation has been investigated by Raweshidy et al. [21]. The despreading is done just after the optical link. In that work, a comparison is made with the network with no fiber link and shows good improvement. Another work [22] proposes the spreading as a form of multi-cell-access scheme where each RAP is assigned a unique PN code and the fibers are interconnected to form a bus network.

\subsubsection{What is Missing?}

In general, the approaches to solve the NLD can be categorized into three groups. The first category attempts to compress the signal to stay within the linear range. This approach does not solve the phase nonlinearity problem and demands more (linear) amplification at the receiver.

Let us see what is the phase nonlinearity problem. In wireless communications, the information is transmitted by phase modulation of the microwave signal. As a result of the stimulated emission mechanism in the laser diode, the time delay of electron to photon conversion process varies as a function of instantaneous driving current. Therefore, even in the so-called linear region of the ROF link, the phase response of the link is nonlinear. Currently, there is no device independent model available to estimate this phase response of the ROF link.

The second approach is modeling the laser. This approach would yield accurate results if the physical parameters of the particular device were accurately measured. Besides, with this approach other nonlinear elements of the link also have to be 
adequately modeled. The third is the network layer approach. Usually, it is best solving the problem in the physical layer itself without transferring the issue to higher layers.

In this thesis, we propose to investigate digital-signal-processing (DSP) techniques for fiber-wireless systems. A question may arise here, why DSP techniques are not popular for optical links. The answer is, most of the research in fiber-optic communications is dedicated to digital fiber links. Electronic DSP techniques are not feasible with the digital optical links. If we consider analog fiber optic links, these are mainly used in CATV applications where, only the downlink or a broadcasting architecture is used. There is no wireless channel involved and the dynamic range requirement is not that severe. Besides, the TV signal itself is analog and so analog (electronic) compensation schemes are preferred.

However, DSP solutions are ideal for fiber-wireless systems. This is because, the ROF links for wireless access is a unique area that emerged very recently. In this scenario, although the radio frequency is in $\mathrm{GHz}$ range, the baseband symbol rate is only a few Mega symbols per second. These vector-modulated symbols carry all the information and all the impairments of the ROF link finally reflects on the phase and amplitude distortions of these symbols. Thus, it is more convenient to implement DSP solutions that run at baseband symbol rates to mitigate ROF channel impairments.

According to the author's knowledge, there is no work reported so far that investigates a multipath wireless channel in series with the nonlinear fiber channel. This makes the work reported in this thesis unique. Only a unified model can provide a unified solution, which would be the optimum.

\subsection{Major Contrbutions of the Dissertation}

It is surprising to see that most of the attempts so far reported are based on either electronic circuit design approaches or some unconventional technique to reduce the dynamic range of the input RF signal. A universal model of the ROF link that incorporates all the device dependent parameters is missing. Once this model is developed, it is possible to explore a suitable signal-processing scheme for performance 
improvement.

The first phase of this dissertation is focused on completely understanding the ROF link and deriving a mathematical model. Then baseband compensation schemes are developed for the ROF link assuming an AWGN wireless channel. A baseband approach is preferred because it is independent of the radio and optical frequencies and it enables easy deployment of adaptive schemes. Adaptive schemes are desirable to accommodate links with large variations. Since, adaptive filters train themselves from the input/output symbols, no accurate knowledge of link physical parameters is required. It is also easy to track any modification or drifts in the link parameters.

\subsubsection{Baseband Modeling}

In this thesis, a mathematical model is first developed for the radio-over-fiber link for wireless access. This model is based on both theoretical studies and on experimental measurements. Then, an adaptive modeling scheme is derived by configuring Volterra series adaptive filters to learn the ROF link from input output baseband data [23]. These adaptive filters are then used to represent the ROF link in the analysis and in simulations.

\subsubsection{Adaptive Predistortion}

Two baseband predistortion schemes are proposed for the downlink. With the first scheme, the predistortion is done using a look-up table [24]. In the second scheme, higher order adaptive filters are trained to inverse model the ROF link [25]. With both of these approaches, simulation results show very good performance improvement for the phase compensation. For amplitude compensation, however, some back-off is required to protect the laser diode from excessive input power.

\subsubsection{Adaptive Post-Compensation}

A post compensation scheme is proposed for the uplink. The scheme consists of higher order adaptive filters located in the central base station to compensate for the nonlinear distortion of the ROF link. With this scheme the saturating type 
amplitude distortion is completely compensated. However, the phase compensation depends on the input dynamic range [26].

\subsubsection{Asymmetry In Complexity}

Asymmetry in complexity is achicved by doing predistortion for the downlink and post compensation for the uplink. That is, most of the signal processing is done at the central base station. This is desirable for cost sharing and there are other benefits. The issues associated with this asymmetric arrangement are discussed in detail and a unified analysis is carried out in [27].

\subsubsection{A Joint Solution}

In addition to the nonlinear distortion, multipath dispersion of the wireless link is also a major factor that limits the system performance. Almost all of the previous work, including the two stage cancellation approach in [19] focused on the ROF link assuming, an AWG.N wireless channel. This is because of the difficulties associated with, dealing the time dispersive fast varying wircless channel in series with the nonlinear ROF channel. The major contribution of this disscrtation is the development of an efficient algorithm for the estimation of the combined fiber-wireless channel as well as the development of a unique receiver architecture for this channel.

\subsubsection{Estimation of the Fiber-Wireless Channel}

The fiber-wireless uplink consists of a linear dynamic system (the wircless channel) followed by a static nonlinear system (the ROF link). 'Therefore, it can be modeled as a Wiener system for Gaussian inputs, after reputed mathematician N. Wiener. Wiener first showed that any BIBO stable nonlinear system with finite memory can be modeled as a Wiener system for Gaussian inputs which, consists of orthogonal linear dynamic functions followed by static nonlinear functions [28].

However, due to the practical difficulties in generating Gaussian inputs, different approaches have been proposed. Pseudonoise (PN) sequences have white noisc like properties and, easy to generate and analyze. Their correlation properties are woll understood [29]. Besides, maximal length PN sequences are widely used in spread 
spectrum communications. Therefore, using PN sequence for channel estimation is very attractive in wireless communications. Billings and Fakhouri initially used PN sequences for control system identification [30].

Following that work we developed a unique algorithm that estimates both the nonlinear transfer function of the ROF link plus the impulse response of the wireless channel individually [31]. Correlation properties of pseudo noise (PN) sequences are used for this estimation. An efficient, Vandermonde matrix approach is used to separate the Volterra kernels of the fiber-wireless channel which, eliminates the computation of higher order correlation functions. Furthermore, projection of linear and nonlinear coefficients onto different subspaces makes the tracking of fast changing wireless channel and the relatively static nonlinear channel virtually independent.

\subsubsection{A Hammerstein type Decision Feedback Equalizer}

Once the channel is estimated, an appropriate equalizer is devised for the compensation of the combined fiber-wireless channel. Mathematically, the structure of an equalizer should be an exact inverse of the channel structure. The fiber-wireless uplink is modeled as a Wiener system and the inverse of a Wiener system is a Hammerstein system. Furthermore, the fiber-wireless channel has the following properties:

1. The wireless channel varies relatively fast. This implies that the compensation should follow it in real time.

2. The nonlinearity comes from the laser diode and from the RF amplifier. Hence, it is almost stationary. That means the nonlinear compensation need to be updated only occasionally.

3. When multiple users are supported by this fiber based wireless access scheme, each user has a different wireless channel. But all of them will share the same ROF channel.

From the foregoing, it is logical that an efficient equalizer should separately compensate for linear and nonlinear distortions. A receiver that accomplishes this intention is proposed in this dissertation. The proposed receiver consists of a polynomial filter 
which, inverse models the optical link nonlinearity, and a linear DFE arrangement that compensates for the wireless channel dispersion. This arrangement also enables a single polynomial filter to be used for all the users in a multiuser environment. We have theoretically optimized the filter parameters and have done a performance analysis [32].

\subsection{Organization}

The rest of this thesis is organized as follows. In Chapter 2, characteristics of each element in the ROF link are investigated in detail. This includes nonlinearity of the laser diode, chromatic dispersion effects of the fiber and various noise mechanisms in the link. Previously reported results are reviewed whenever appropriate. Preliminary simulation results are also included.

Chapter 3 describes the empirical characterization of the ROF link. Both basic measurements using standard test equipment as well as advanced measurements using the 'impulse response instrumentation system' (IRIS) available at TRLabs are described, Then we identify the key parameters of the ROF link that cause the major impairment. This is done from both theoretical and empirical characterization.

A mathematical background on nonlinear systems and Volterra series adaptive filters is given in Chapter 4. The baseband modeling scheme as well as the look-up table and higher order adaptive filter based compensation schemes are described. Various issues of doing this compensation either before or after the link are examined. Both theoretical and simulation results are given.

Chapter 5 analyzes the bit error rate performance of the fiber-wireless channel, transmitting a direct-spread code division multiple access scheme (DS/CDMA) signals. Various optical and wireless channel noise plus the multiuser interference is considered in the BER analysis.

From Chapter 6 onwards multipath dispersion of the wireless channel is considered along with the nonlinear distortion of the fiber channel. In Chapter 6 , Wiener/Hammerstein system based modeling of the fiber-wireless channel is described. Then a PN sequence correlation technique to estimate this channel is mathematically derived and simulation results are provided. In Chapter 7, a uni- 
fied compensation scheme is described, by introducing the Hammerstein type DFE (HDFE). The HDFE parameters are theoretically optimized to minimize the MSE. In Chapter 8, the performance of the HDFE is analyzed under various operating conditions. Finally, Chapter 9 concludes the dissertation by summarizing the results and outlining possible future research directions. 


\section{Chapter 2}

\section{The Radio Over Fiber Link}

The radio over fiber link was introduced and its various characteristics have been briefly outlined in Chapter 1. In this chapter, an in-depth discussion of a directly modulated ROF link is presented. This is done both by theoretical analysis and by empirical measurements. At the end of this chapter it will be clear that some link parameters are critically important for transmitting radio signals for wireless access, while, others turn out to be less significant.

The ROF link consists of three main elements: an optical source (laser) with an RF-optical modulating arrangement, a single mode fiber link and an optical receiver with a band pass filter to recover the original signal. In addition, an RF amplifier is often included at the optical receiver. Each of these elements is reviewed in detail in this chapter. Characterization through empirical measurements is described in the next chapter.

\subsection{The Semiconductor Laser}

Although there are numerous types of lasers that are used as light sources, here, we confine our discussion to high-speed semiconductor lasers, that could be coupled with optical fibers. Even under this category, there are two types of lasers that are mainly used in analog application. This is because, for low distortion transmission at $\mathrm{RF}$ frequencies, the laser should have a very narrow linewidth, so that they can be approximated as a single mode laser. These two types are described below, 
Fabry-Perot Laser This laser utilizes the reflection from the laser facets to provide the feedback necessary for the lasing process. Although narrow linewidth postulates single mode emission, typically the Fabry-Perot laser lases in many longitudinal modes (referred to as multi longitudinal mode lasing) instead of a single mode. This gives a wider line width that can cause more distortion while propagating in the fiber due to chromatic dispersion. Also the FabryPerot laser shows a phenomenon called 'mode hopping' in which the power constantly shifts between the modes. Therefore, the Fabry-Perot laser is subjected to both mode hopping and mode partition noise. These characteristics usually limit the Fabry-Perot laser to less demanding applications.

Distributed Feedback (DFB) Laser In the DFB laser, the feedback is obtained from the distributed Bragg diffraction grating rather than by the usual cleaved mirrors. Due to this special grating pattern, only one lasing mode is constructively created. Thus the DFB is a single mode laser and less subjected to fiber distortion. Also it provides more dynamic range and the emission is relatively less distorted. These desirable characteristics make the DFB, the most widely used laser in analog applications.

\subsubsection{Nonlinearity of the Laser}

Lasers are inherently nonlinear. The very basic lasing operation involves several nonlinear mechanisms such as the 'threshold current' and the 'stimulated emission'. The stimulated emission is a self-multiplying mechanism that occurs under positive optical feedback conditions. Spontaneously emitted photons lack coherency and therefore not desirable. However, only when the driving current is larger than the threshold current, the stimulated emission becomes dominant compared to the spontaneous emission process.

Nevertheless, under very confined conditions, there are tireless efforts to use lasers in linear application. In this case, however, the nonlinearity of the laser mainly limits the spurious free dynamic range (SFDR) or shortly, the dynamic range (DR). This is the range of input RF power for which the linear output terms are above the noise floor and the intermodulation products are below the noise floor. 


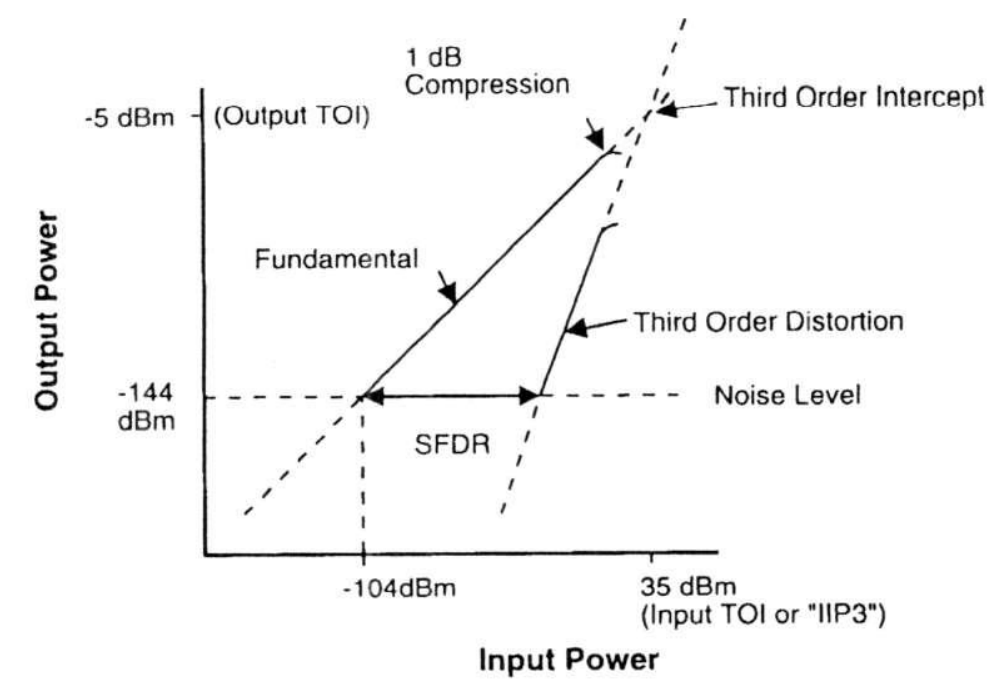

Figure 2.1: Basic parameters for characterizing a nonlinearity

\subsubsection{Characterization of the Nonlinearity}

When the laser is driven by an RF signal, the nonlinear distortion of the output depends on the number of distinguished spectral components that are present in the RF signal. The basic parameters that characterize a nonlinearity are shown in Fig. 2.1. Here, TOI is the third order intercept point which is also refered to as input intercept point of order three (IIP3).

Just one tone is present. Here, the one- $\mathrm{dB}$ compression point is used as a reference to measure the nonlinearity. The one- $\mathrm{dB}$ compression point is defined as the input power that gives an output power less than one- $\mathrm{dB}$ of the output power if the laser would have been perfectly linear. In other words, at this point the output power deviates by one- $\mathrm{dB}$ from the linearly predicted value. When baseband information is modulated on the RF signal, the one-dB compression point manifests itself as an AM-AM type distortion.

Two tones are present: The third order intercept point (TOI) is the key measure of nonlinearity when two tones are present. The third order intercept point is defined as the input power at which the extrapolated slopes of the output signal and the distortion terms intercept. That is, at this point the intermodulation term would have the same power as the linear term. This phenomenon 


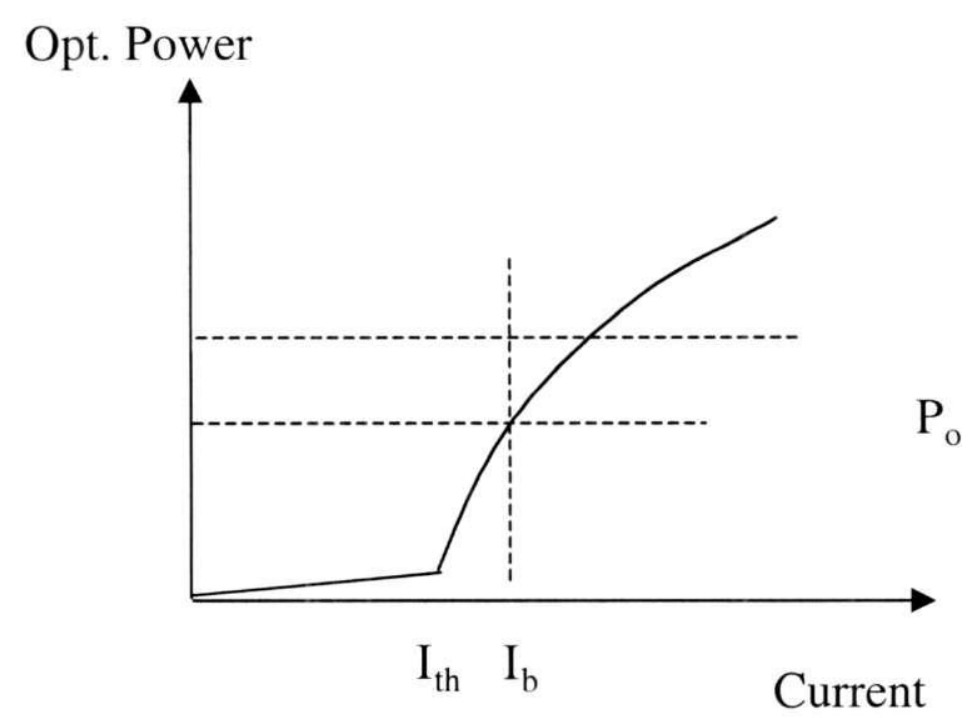

Figure 2.2: Electrical to optical conversion curve in a typical semiconductor laser occurs at a higher power level than the one-dB compression point. The maximum operating power of a laser is typically at a much lower level than the third order intercept point.

Large number of carriers: When large number of carriers are present, many intermodulation terms would exist giving higher order intermodulation distortion. Odd order intermodulation products are of major concern since, they tend to overlap with the linear terms in a typical band pass system.

\subsubsection{Static and Dynamic Nonlinearity}

From the point of view of time domain characteristics of the input signal, there are two types of nonlinearities, which are fairly independent of each other.

\section{Static Nonlinearity}

The injected current versus optical power characteristics of the semiconductor laser is shown in Fig. 2.2 which, is generally referred as the P-I curve. From Fig. 2.2, it can be seen that the laser starts lasing above the threshold current $I_{t h}$. The P-I curve is not quite linear after the threshold point, though often linearity is assumed. This curve is typically expressed by a third order polynomial [9]. This is referred 
to as the static nonlinearity or the AM-AM gain compression, which is memoryless. The second order distortion is proportional to the first derivative, $d P / d I$, and the third order distortion is proportional to second derivative, $d^{2} P / d I^{2}[33]$. The third order polynomial approximation that is widely used in the literature is given in (2.1) where, $s(t)$ is the input current, $m$ is the DC modulation depth, $P(t)$ is the instantaneous optical power emitted from the laser and $P_{o}$ is the mean optical power. By measuring intermodulation and harmonic components using multi-tone test methods, the coefficients of this third order laser transfer curve can be found.

$$
P(t)=P_{o}\left[1+m s(t)+A_{2} m^{2} s^{2}(t)+A_{3} m^{3} s^{3}(t)\right]
$$

The optical modulation depth $m$ is defined as the ratio between the peak optical power and the average optical power $\left(P_{o}\right)$ (Fig. 1.2). Generally, the lower the $m$, the less the nonlinear distortion. However, a lower $m$ also reduces RF subcarrier power and signal to noise ratio. Higher $m$, on the other hand, increase the probability of clipping. This suggests that an optimum $m$ can be found for a link based on the particular link characteristics.

\section{Dynamic Nonlinearity}

Since lasers are dissipative systems, they have asymptotic solutions for their state as time goes to infinity. The solution can be independent of the initial condition [34]. That is, if the transients are ignored, the laser can be described by the asymptotic solutions of its differential equations generally known as laser rate equations. The rate equations govern the dynamic nonlinearity.

In general, the dynamic nonlinearity of a semiconductor laser diode is frequency dependent. However, if the modulating frequency is at least five times less than the laser resonance frequency, then it can be assumed to be frequency independent [7]. In any case, under transient and fast varying signal conditions, the dynamic nonlinearity plays a key role.

The laser rate equations that govern the nonlinear electron to photon conversion process are given in (2.2) and (2.3) and a description of each parameter is given in Table 2.1. Optical confinement factor is defined as the ratio between the active 


\begin{tabular}{|c|c|}
\hline$N_{e}$ & Electron Density \\
\hline$N_{p}$ & Photon Density \\
\hline$\Gamma$ & Optical Confinement Factor \\
\hline$g_{o}$ & Gain Slope Constant \\
\hline$N_{o g}$ & Plectron density at which the net gain zero \\
\hline$\tau_{p}$ & Electron lifetime \\
\hline$\tau_{n}$ & Volume of the active layer \\
\hline$\beta$ & Eraction of the spontaneous emission coupled into laser mode \\
\hline$V_{a c t}$ & Current Injected into the active layer \\
\hline$q$ & Gain compression coefficient of the active region \\
\hline$I_{A}$ & \\
\hline$\varepsilon$ &
\end{tabular}

Table 2.1: Parameters of the laser rate equations

region volume and the modal volume.

$$
\begin{gathered}
\frac{d N_{e}}{d t}=\frac{I_{A}}{q V_{a c t}}-\frac{N_{e}}{\tau_{n}}-g_{o}\left(N_{e}-N_{o g}\right)\left(1-\varepsilon N_{p}\right) N_{p} \\
\frac{d N_{p}}{d t}=\left[g_{o}\left(N_{e}-N_{o g}\right)\left(1-\varepsilon N_{p}\right) N_{p}-\frac{N_{p}}{\tau_{p}}+\beta \frac{N_{e}}{\tau_{n}}\right] \Gamma
\end{gathered}
$$

\subsubsection{Laser Clipping}

Depending on the instantaneous amplitude of the RF signal and the value of bias current $I_{b}$, the output optical signal gets clipped when the negative peak goes below the threshold (See Fig. 2.2). Generally, this is referred to as clipping noise that is an impulsive type of noise. The probability of clipping is especially high with multiple $\mathrm{RF}$ carriers. If the input to the laser $s(t)$ is a stationary zero mean Gaussian process, the output $P(t)$, considering clipping noise $n_{c l}(t)$ is,

$$
P(t)=P_{o}\left[1+K_{c l} m s(t)+n_{c l}(t)\right]
$$

where, $K_{c l}$ is the attenuation due to clipping. Conradi and Lai [35] have done experimental analysis on clipping noise. The clipping rate asymptotically becomes a Poisson process. The probability density function of the clipping interval asymptotically becomes a Rayleigh density function. Finally, the shape of the impulse over the impulse duration can be approximated by a parabolic function. 


\subsubsection{Natural Frequency}

The natural frequency is the frequency at which a relaxation oscillation of the laser occurs after a state transition. The natural frequency limits the highest frequency at which the laser can be modulated. A resonance occurs when the modulating frequency is same as the natural frequency. Therefore, this is also referred to as the resonance frequency $\left(f_{r}\right)$. This is normally about $22 \mathrm{GHz}$ and is proportional to the square root of the average optical power in analog applications [12]. Typically, the modulating frequency is kept well below the resonance frequency. However, in the literature a record $33 \mathrm{GHz}$ direct modulation is reported [6] by having a driving current 10-12 times higher than the minimum lasing current. This pushes the $f_{r}$ further up. However, at this super high modulating frequency, other characteristics of the laser deteriorate. In any case, the point of interest is that a laser can be directly modulated up to several $\mathrm{GHz}$ easily.

\subsubsection{Small Signal Analysis}

When the modulation depth is small, the laser output can be characterized by performing a small signal analysis. By performing a small signal analysis on the laser rate equations, Tucker et al. [13], [36] have obtained expressions to show the intermodulation and harmonic distortions in terms of modulating frequency $(f)$, resonance frequency $\left(f_{r}\right)$, gain compression characteristics and the modulation depth $m$ of the laser. Also, they have verified these expressions experimentally. The variation in gain compression is the main reason for the device dependency of distortion characteristics. This is further investigated by W. I. Way [9] where he included various noise terms of the link to get expressions for the spurious free dynamic range (SFDR) of the link, which is given below in $\mathrm{dB}$.

$$
S F D R=\frac{2}{3} \log _{10}\left[I_{D}^{2} /\left|\left(\frac{f}{f_{r}}\right)^{4}-\frac{1}{2}\left(\frac{f}{f_{r}}\right)^{2}\right|\left(R I N * I_{D}^{2}+2 q I_{D}+\left\langle i_{n}^{2}\right\rangle\right)\right]
$$

Here, $I_{D}$ is the detector current (proportional to optical power), $\left\langle I_{t h}^{2}\right\rangle$ is the receiver thermal noise (independent of optical power), $I_{R I N}$ is the laser relative intensity noise (proportional to the square of optical power). 
The following observations can be made from the expression in (2.5).

- If the dominant noise term is RIN, then the SFDR is independent of optical power.

- If the dominant noise term is the shot noise $\left(2 q I_{D}\right)$ then SFDR is linearly dependent on optical power.

- Usually, thermal noise is less significant. However if it is not, then the SFDR depends on $I_{D}^{2}$.

In all these cases SFDR is also a function of laser resonance frequency. The higher the resonance frequency, the better the dynamic range. The resonance frequency can be pushed up by increasing the bias current to a certain extent [6]. However, this would also increase the nonlinearity by increasing the leakage current. The leakage current is defined as the current that passes through the resistive elements of the laser and does not contribute to photon generation. In current devices, the resonance frequency is inherently limited to about $22 \mathrm{GHz}$ by these factors, see section 2.1.3.

\subsubsection{Small Signal Frequency Response}

Theoretical analysis has shown that the intrinsic modulation response of a laser diode is similar to a second order low pass network [12]. The response exhibits a resonance peak at $f_{r}$ before rolling down at $40 \mathrm{~dB} /$ decade. Usually, the higher the gain of the resonance peak, the more nonlinear is the phase response.

\subsubsection{Large Signal Analysis}

The above discussion is valid for small signal analysis or when the modulation depth $m$ is less than 0.3 . Under large signal conditions several additional factors need to be considered that are unknown and nonlinear. The best approach is to use an equivalent circuit approach. Several such circuits have been investigated [13], [36]. These circuits can then be analyzed using an electronic circuit analysis program such as Pspice ${ }^{T M}$. However, determining all the circuit parameters is usually a tedious process. Applying curve fitting algorithms on measured characteristics is 
used to determine some parameter values. In addition, the parameters are device dependent. This is one major reason for the motivation in this thesis to use adaptive filters to model the entire ROF link from its input/output data. This avoids the tedious process of characterizing a laser from its physical parameters.

\subsubsection{Laser Chirp}

When the optical intensity is modulated, inevitably (the phase or) the frequency of the optical carrier is also modulated. This unwanted frequency modulation is called the chirp. Because of the chromatic dispersion of the fiber, different frequency components travel with different speeds. Therefore, the chirp is converted into undesirable amplitude distortion at the far end.

However, there is an uncxpected benefit from the chirp. Higher chirp reduces the effect of the noise because the total noisc powcr is spread over a frequency range, which is proportional to the chirped line width. The chirped line width is much larger than the original line width. Therefore, for a given noise power, the higher the chirp, the lower the noise-power per unit bandwidth. From this mechanism, it can be seen that the distortion increases with increasing chirp, but noise within the passband decreases. This means there is an optimum chirp range for lincar DFB lasers in broad band links. At $1550 \mathrm{~nm}$, chirp can also be used to compensate the effects of fiber dispersion on the sidebands of a modulated (wideband) laser signal

Let us do a quantitative analysis on the effect of chirp in a typical ROF link. The chirp is usually in the range of $50-1.50 \mathrm{MHz} / \mathrm{mA}$. We shall use the worst case chirp to find out the maximum impairment.

From the specifications of the PFT-1700 laser, which is used in our experiment, the spurious free dynamic range is about $40 \mathrm{~dB}$ (approximately () $\mathrm{dBm}$ to $-40 \mathrm{dBm}$ ). In a 50 -ohm system the current varies from $44.7 \mu \mathrm{A}$ to $4.47 \mathrm{~mA}$. At a $150 \mathrm{MHz} / \mathrm{mA}$ chirp gain, this current variation will introduce a change of $675 \mathrm{MHz}$ optical frequency shift which is equivalent to $0.004 \mathrm{~nm}$ optical wavelength shift.

There should be no confusion by comparing this optical frequency shift with the RF carrier frequency $(1.8 \mathrm{GHz}$ ). The RF carrier frequency is the frequency of the cnvelope in the fiber, while the optical frequency shift $(675 \mathrm{MH} \%)$ is the shift from the optical carrier frequency $f_{0}$ which is $229 \mathrm{THz}$. A shift of $0.004 \mathrm{~nm}$ from 
$1310 \mathrm{~nm}$ is insignificant. Thus the chirp will not introduce any significant distortion in the fiber especially in an environment where the manufacturer specified center wavelength itself can be $1310 \mathrm{~nm}+/-10 \mathrm{~nm}$ and the fiber length is very short.

\subsubsection{Relative Intensity Noise (RIN)}

A fluctuation in the optical output intensity due to spontaneous emission that is inherent to lasing mechanism leads to this optical intensity noise. So named because it is the ratio of the mean square amplitude of the noise fluctuations per unit bandwidth $\left\langle\Delta P_{o}^{2}\right\rangle$, to the square of the optical power $P_{o}$ itself. That is, the noise due to RIN is proportional to the square of the optical power. Mathematically this can be written as,

$$
R I N=\left\langle\Delta P_{o}^{2}\right\rangle /\left\langle P_{o}\right\rangle^{2}
$$

RIN is a constant specified for a given laser. Typically RIN range from -130 to -155 $\mathrm{dB} / \mathrm{Hz}$.

\subsubsection{Impedance Matching}

Typically, the laser is a low impedance device (2 to $3 \mathrm{ohms}$ ) because of the current injection. It has to be matched to the $50 \mathrm{ohm} \mathrm{RF}$ system. This matching can be done either resistively or reactively. In the resistive matching technique, a passive matching network consisting of only resistive elements is used. Therefore, this technique provides a wideband solution but has high attenuation properties. In the reactively matched networks, reactive elements such as inductors and capacitors are used, so that there is some gain in the pass band. However, these circuits are relatively narrow band. In any case, we should note that, in the optical world even one $\mathrm{GHz}$ bandwidth is referred to as narrow band. Therefore, for wireless access, reactively matched ROF links are preferred. This is especially true because power link budget of the ROF link is more limited than bandwidth. For example the ROF link used in our experiment offered a $500 \mathrm{MHz}$ bandwidth with reactive matching. 


\subsection{Fiber Dispersion and Distortion Issues}

Single mode (SM) fiber is the best fiber for this application due to its low dispersion characteristics and especially because it does not have modal dispersion. However, even in the SM fiber, the following imperfections exist.

\subsubsection{Chromatic Dispersion}

Chromatic dispersion arises because different frequency components have different velocities in the fiber. The delay differences are caused by the dispersive properties of the fiber material itself. The chromatic dispersion is zero at $1310 \mathrm{~nm}$. That is, if the transmitted signal were an impulse at $1310 \mathrm{~nm}$, there would be no dispersion. When modulated, however, any real source occupies a finite bandwidth. Therefore, there is always chromatic dispersion. In addition, commercially available lasers have a tolerance of up to about $10 \mathrm{~nm}$ off their specified center wavelength $(1310+/-10$ $\mathrm{nm})$.

The high chromatic dispersion is one concern with the 1550 -nm band analog ROF links. However, the $1550 \mathrm{~nm}$ band has low attenuation and has readily available Erbium doped fiber amplifiers. These factors make the $1550 \mathrm{~nm}$ attractive in attenuation limited applications. Therefore, when the chromatic dispersion is not the limiting factor, $1550 \mathrm{~nm}$ is preferred.

\section{Mathematical Analysis of Chromatic Dispersion}

The CCITT has specified a closed form expression for the chromatic dispersion $d(\lambda)$ in a standard SM fiber [37]. This is given by,

$$
d(\lambda)=s_{o}\left(\lambda-\lambda_{o}^{4} / \lambda^{3}\right) / 4
$$

The zero dispersion slope $s_{o}$ is typically $0.087 \mathrm{ps} / \mathrm{nm}^{2} / \mathrm{km}$ and the zero dispersion wavelength $\lambda_{o}$ is $1310 \mathrm{~nm}$. $\lambda$ is the operating wavelength. Note that there is no dispersion if, $\lambda=\lambda_{o}$.

For a DFB laser that has a narrow line width, (the source line width is comparable to the modulation bandwidth), the following low pass equivalent expression 
can be used to represent the transfer function of the fiber [37].

$$
H_{S M F}(f)=\exp \left(-j \alpha l_{f} f^{2}\right)
$$

where, $\alpha=\pi d\left(\lambda_{s}\right) \lambda_{s}^{2} / c, c$ is the speed of light, $\lambda_{s}$ is the source operating wavelength and $l_{f}$ is the fiber length. For example, for a wavelength shift of $1310+/-10 \mathrm{~nm}$, the calculated value for $\alpha$ is $+/-1.6 \times 10^{-23} \mathrm{~s}^{2} / \mathrm{km}$. This is done by taking $\lambda_{s}$ as $1320 \mathrm{~nm}$ and $\lambda_{o}$ as $1310 \mathrm{~nm}$. Therefore $d\left(\lambda_{s}\right)$ can be calculated.

Now, to find the output spectrum $Y(f)$, this transfer function $H(f)$ of the fiber has to be multiplied with the input spectrum $X(f)$. Let the modulating RF signal at the laser be $s(t)$. The $s(t)$ has a carrier frequency of $f_{c}$ and a bandwidth of $2 /$ (bit rate of the information signal). Then, assuming ideal modulation the optical electric field (not the optical power) output of the laser, which is amplitude modulated, is written as

$$
e_{o}(t)=\operatorname{Re}\left\{[1+m s(t)] \exp \left(j 2 \pi f_{o} t\right)\right\}
$$

where $f_{o}$ is the optical carrier frequency and $m$ is the modulation depth of the laser. Taking the Fourier transform of this waveform.

$$
E_{o}(f)=\delta\left(f-f_{o}\right)+\frac{m}{2}\left[S\left(f-\overline{f_{o}-f_{c}}\right)+S\left(f-\overline{f_{o}+f_{c}}\right)\right]
$$

This represents a DSB spectrum with the carrier at $f_{o}$. The side bands $S(f)$ are separated by $f_{c}$ from the optical carrier $f_{o} . f_{c}$ is the carrier frequency of $s(t)$. The bandwidth of $s(t)$ is much smaller than $f_{c}$. A better understanding of this scenario is obtained by referring to Fig. 1.2. The band pass transfer function of the fiber is written as,

$$
H_{b p}(f)=e^{-j \alpha l_{f}\left(f-f_{0}\right)^{2}}
$$

Now, the output optical signal at the end of the fiber is given as

$$
\begin{gathered}
Y_{b p}(f)=H_{b p}(f) E_{o}(f) \\
Y_{b p}(f)=\delta\left(f-f_{o}\right)+\frac{m}{2}\left[S\left(f-\overline{f_{o}-f_{c}}\right)+S\left(f-\overline{f_{o}+f_{c}}\right)\right] e^{-j \alpha l_{f} f^{2}}
\end{gathered}
$$

The detector is a square law device. Therefore, the photocurrent is proportional to 
the square of the optical field. Note that the square of the optical field is proportional to the optical power.

$$
I(t) \propto\left|y_{\iota p}(t)\right|^{2}
$$

Squaring in the time domain then converting to the frequency domain and band pass filtering at $f_{c}$ is same as auto correlating in the frequency domain with a frequency shift of $f_{c}$. Using this fact, it can be shown that the signal power following self homodyne detection $W$ at the detector, as a function of fiber length $l$ is,

$$
W(l) \propto \cos ^{2}\left[\alpha l_{f} f_{c}^{2}\right]
$$

The complete proof of (2.15) is given in [5].

Equation (2.15) is a simple expression that shows the received optical power as a function of chromatic dispersion, radio frequency and the fiber length. From this expression, for a given $\alpha, l_{f}$ and $f_{c}$, the term $\cos ^{2}\left(\left(l_{f} f_{c}^{2}\right)\right.$ can become zero. This is called the sideband cancellation effect. At UHF frequencies, when the wavelength is $1550 \mathrm{~nm}$, and the fiber length is very long, this can become a serious drawback. Nevertheless, in PCS band with $1310 \mathrm{~nm}$ wavelength this is not a concern as shown below.

$\alpha$ is a function of wavelength. At $1310+/-10 \mathrm{~nm},|\alpha|=1.6 \times 10^{-23} \mathrm{~s}^{2} / \mathrm{km}$ and if $f_{c}=1.8 \mathrm{GHz}$, then $f_{c}^{2}=51.84 \times 10^{-6} / \mathrm{km}$ and $W(l=1)$ is almost one. Or with precise substitution, it will take $30,000 \mathrm{~km}$ before we see a null. So the effect of sideband cancellation is negligible in our application.

This is also apparent from Fig. 2.3 that shows the frequency and wavelength dependency of the sideband cancellations. From the lower figure we can see even at $1550 \mathrm{~nm}$ with $f_{c}=1.8 \mathrm{GHz}$, the sideband cancellation effect comes into play at fiber lengths greater than $\approx 300 \mathrm{~km}$. At $1310 \mathrm{~nm}$, this length is $\approx 3,000 \mathrm{~km}$ However, typical distances from the central base station to radio access points are much shorter than this.

By going one step further, the effect of chromatic dispersion on the RF bandwidth also is calculated. In other words, we estimate the difference in the frequency dependent attenuation at $1.7 \mathrm{GHz}$ and at $2.2 \mathrm{GHz}$ because our operating signal band is from $1.7 \mathrm{GHz}$ to $2.2 \mathrm{GHz}$. This is done by computing $W(l)$ at $2.2 \mathrm{GHz}$ and 

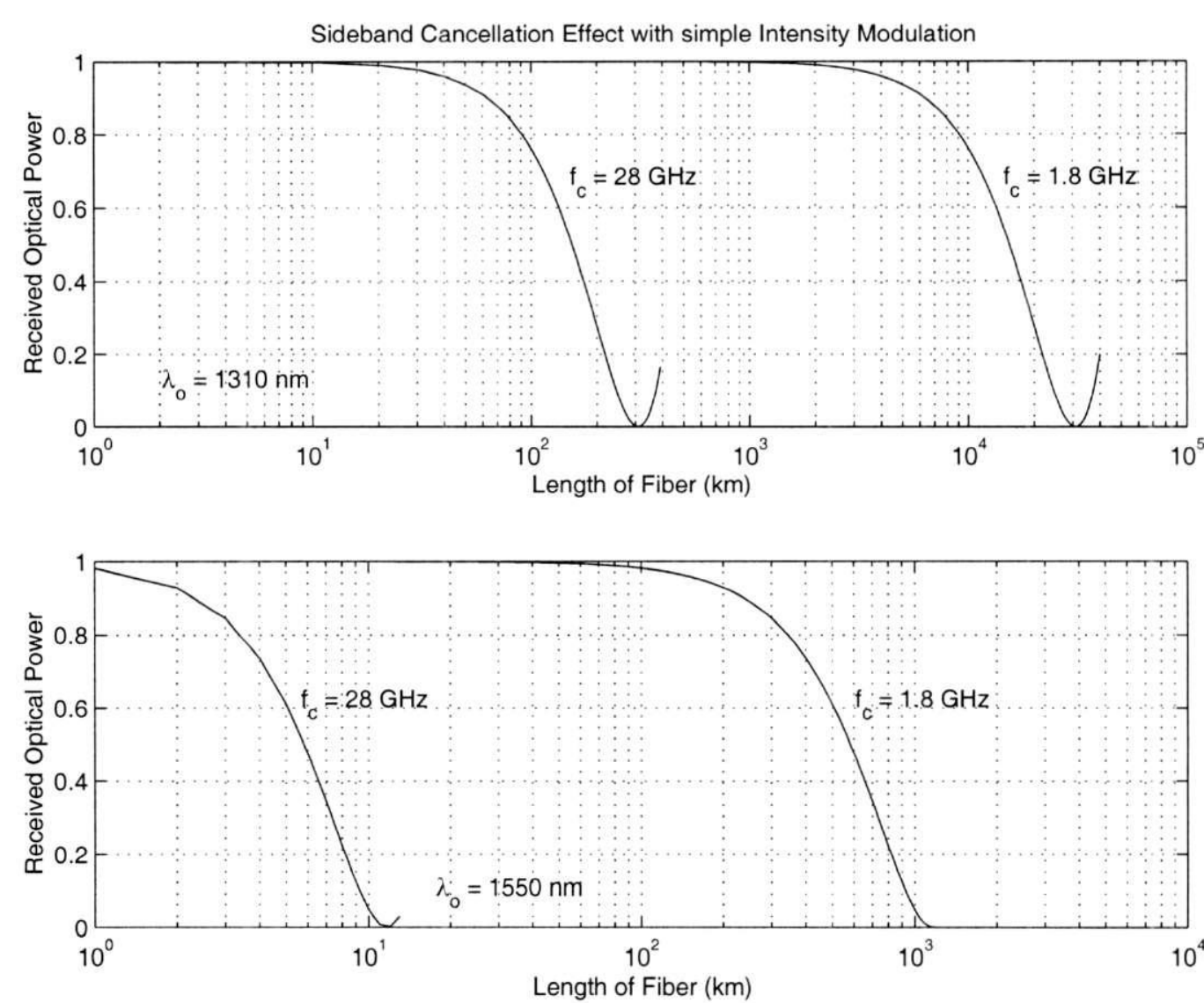

Figure 2.3: Sideband cancellation effect due to chromatic dispersion

then subtracting it from the $W(l)$ at $1.7 \mathrm{GHz}$. This gives a differential attenuation of $5.17 \times 10^{-9} / \mathrm{km}$, which is same as $-83 \mathrm{~dB} / \mathrm{km}$. This is negligible compared to the common mode attenuation of $0.4 \mathrm{~dB} / \mathrm{km}$, typical for a modern fiber. Therefore, the effect of chromatic dispersion is totally negligible in our application.

\subsubsection{Polarization Mode Dispersion (PMD)}

Polarization mode dispersion arises in a single mode fiber when the combined effects of non-circular symmetric internal stresses and wave guide geometry created during manufacturing process cause the two polarization modes of the wave guide to propagate with different group velocities [38].

This can lead to a second order distortion from two mechanisms. The first mechanism is that the propagation time changes because of the polarization state of the 
light through the fiber changes. The second mechanism is due to any polarization sensitive components in the link. The second process can be avoided by not using any such components. Nevertheless, in modern fibers the polarization mode dispersion, even due to the first mechanism, is not too serious due to improved manufacturing capabilities. This is especially true when the fiber is short.

\subsubsection{Interferometric Noise (IN)}

Interferometric noise can appear in an optical system when the received signal is accompanied by a weak delayed replica of itself or other light wave components. These doubly reflected signals mix electrically with the original signal and cause excess noise [39]. Reflections arise either from discrete reflectors such as splices and connectors or by Rayleigh scattering within the fiber itself.

Basically when the fiber has poor connectors or very long with high optical power, the IN becomes significant. For the fiber lengths less than $20 \mathrm{~km}$ the Rayleigh scatter introduced interferometric noise is negligible [40]. Furthermore, if the number of connectors that have a back reflection factor of $-35 \mathrm{~dB}$ or better is less than 17 , then the discrete reflection effect is also negligible [8].

\section{$2.3 \quad$ Optical Receiver}

The receiver is inherently wideband and cost effective compared to the laser in the analog fiber optic link. Typically the performance of commercial receivers is adequate for most ROF applications. However, for the sake of completeness, let us briefly review the concerns of optical receivers.

Noise, nonlinear responsivity at high power levels, and frequency response (in the desired band) are the primary concerns with optical receivers. At this point it is relevant to mention that the average optical power emitted in to the fiber is constant in a direct modulated ROF link despite the variations in the RF power. The optical power output is only proportional to the DC bias current, which is typically kept constant. With typical modulation depths (say at 0.3 ), the peak of the modulated optical intensity does not exceed $30 \%$ of the mean value.

Higher bandwidth detectors, though commercially available, come with a penalty 
of low responsivity. This is because high bandwidth detectors tend to have smaller photosensitive areas, which limit the power conversion efficiency. On the other hand, large area detectors have high junction-capacitance, which limits the bandwidth. Furthermore, even the same photo detector is more nonlinear at higher frequencies than at low frequencies. For example, high power detectors with a maximum photocurrent of $150 \mathrm{~mA}$ have only about $295 \mathrm{MHz}$ bandwidth, while high-speed detectors with a $50 \mathrm{GHz}$ bandwidth have only about 1-2 mA photocurrent, as reported in $[12]$.

\subsubsection{PIN vs Avalanche Photo Diode}

Two type of detectors, namely the p-type-intrinsic-n-type (PIN) and the avalanche photo diodes (APD), are mostly used in fiber optic receivers. As the name implies the APD has a self-multiplying mechanism so that it has high gain. The drawback of this gain is, the noise also gets multiplied with this process which leads to the so-called 'excess noise'. Compared to short wavelengths (say $800 \mathrm{~nm}$ ), at high wavelengths (say 1310 and $1550 \mathrm{~nm}$ ), APD's have the same excess noise, but they have an order of magnitude lower avalanche gain. Therefore, APD's are not commonly used in long wavelength applications [12]. PIN diodes are widely used at this wavelength region. Therefore, we focus on PIN diode receivers in this work.

In a typical PIN diode receiver, there are three major noise mechanisms. Namely shot noise, thermal noise and the dark current noise. All these noise mechanisms are unavoidable. However, their relative importance depends on a particular design

\subsubsection{Shot Noise}

Light is composed of photons, which are discrete packets of energy. Thus, the randomness of the arrival time of each photon generates a random noise component at the output current of the photo diode which, is referred to as the shot noise. The shot noise is proportional to the average value of the optical signal. The shot noise power is given by,

$$
\left\langle I_{\text {shot }}^{2}\right\rangle=2 q \Re P_{o} B=2 q I_{D} B
$$


where, $P_{o}$ is the optical power at the detector, $q$ is the charge of an electron, $B$ is the bandwidth of interest and $\Re$ is the photo diode responsivity. The detector current, which is denoted by $I_{D}$, is responsivity times $P_{o}$. That is, $I_{D}=\Re P_{o}$.

\subsubsection{Thermal Noise}

Thermal noise is due to the resistive elements in the receiver amplifier. The thermal noise is independent of the optical signal level but increases with the temperature. The thermal noise power is given by,

$$
\left\langle I_{t h}^{2}\right\rangle=4 k_{B} T_{o} B / R_{L}
$$

where, $T_{o}$ is the absolute temperature in Kelvin and $k_{B}$ is the Boltzman constant and $R_{L}$ is the receiver load impedance.

\subsubsection{Dark Current Noise}

Even in absolute dark, there is a very small current from the photodiode due to various leakage effects. The noise power associated with this is given by,

$$
\left\langle I_{\text {dark }}^{2}\right\rangle=2 q I_{\text {dark }} B
$$

However, as we will see later, typically this noise is negligible compared to other noise.

Usually the combination of all these noises is specified by the manufacturer that is called EIN, i.e. equivalent input noise. For example, the link with PFT1700 transmitter and PFR-1701 receiver and 9/125 fiber of $1 \mathrm{~km}$, the total EIN is specified as $-125 \mathrm{dBm} / \mathrm{Hz}$. This has to be multiplied with the used bandwidth to obtain actual noise power.

\subsection{Power Link Budget}

The ROF link has a severely limited power link budget. The limitations come from various reasons, mainly due to optical to electrical conversion and vice versa. 
We derived a power budget equation considering both input output matching and optical-electrical conversion process, which is given below,

$$
G_{\text {link }, d B}=20 \log _{10}\left(G_{m} \Re / 0.001\right)+10 \log _{10}\left(Z_{\text {out }} / Z_{\text {in }}\right)-2(O L)
$$

where, $G_{l i n k, d B}$ is the optical link gain in dB, $G_{m}$ is the modulation gain of the laser diode, $\Re$ is the responsivity of the photodiode, $Z_{\text {out }}$ is the RF impedance at the output of the link and $Z_{\text {in }}$ is the RF impedance at the input of the link. If these are same (say, it is a $50 \mathrm{ohm}$ system everywhere), then they cancel out. $O L$ is the optical link losses. The $O L$ includes fiber attenuation (typically $0.5 \mathrm{~dB} / \mathrm{km}$ ) and loss at optical couplers, splitters etc. Note the loss in the optical domain appears twice in the electrical domain because, after square law detection the output current is proportional to the optical power.

If we consider optical to electrical and electrical to optical conversion only, there is a loss of about $39 \mathrm{~dB}$ (assuming $G_{m}=0.12 \mathrm{~mW} / \mathrm{mA}$ and $\Re=0.75 \mathrm{~mA} / \mathrm{mW}$ ). With reactive matching, an additional gain up to $19 \mathrm{~dB}$ is possible [41].

In addition to this, the modulation depth $m$ at the optical transmitter should be kept small (typically $\leq 0.3$ ), to avoid excessive nonlinear distortion. As a result, the received $\mathrm{RF}$ power at the optical receiver is very low, typically less than $-30 \mathrm{dBm}$. This has to be significantly amplified (by 30-40 dB) before driving an antenna even in a microcellular scenario. For this reason, illuminating a macrocell with RAP is almost impossible without very high gain RF amplifiers.

\subsection{Preliminary Simulations}

The preliminary simulation is used to get a quantitative feeling of the link. Simulink ${ }^{T M}$ dynamic system simulator running in Matlab ${ }^{T M}$ environment is used for simulation.

The first challenge is to run a simulation at $1.8 \mathrm{GHz} \mathrm{RF}$ carrier frequencies. Typically, the baseband equivalent model is used to avoid running the simulation at the RF carrier frequency. However, in our case, the optical envelope is in the radio frequency and the information is at base band. In other words there is a twostage frequency translation. Therefore, we cannot use a complex low pass model and the simulation has to be in radio frequency. Hence, we decided to use a 


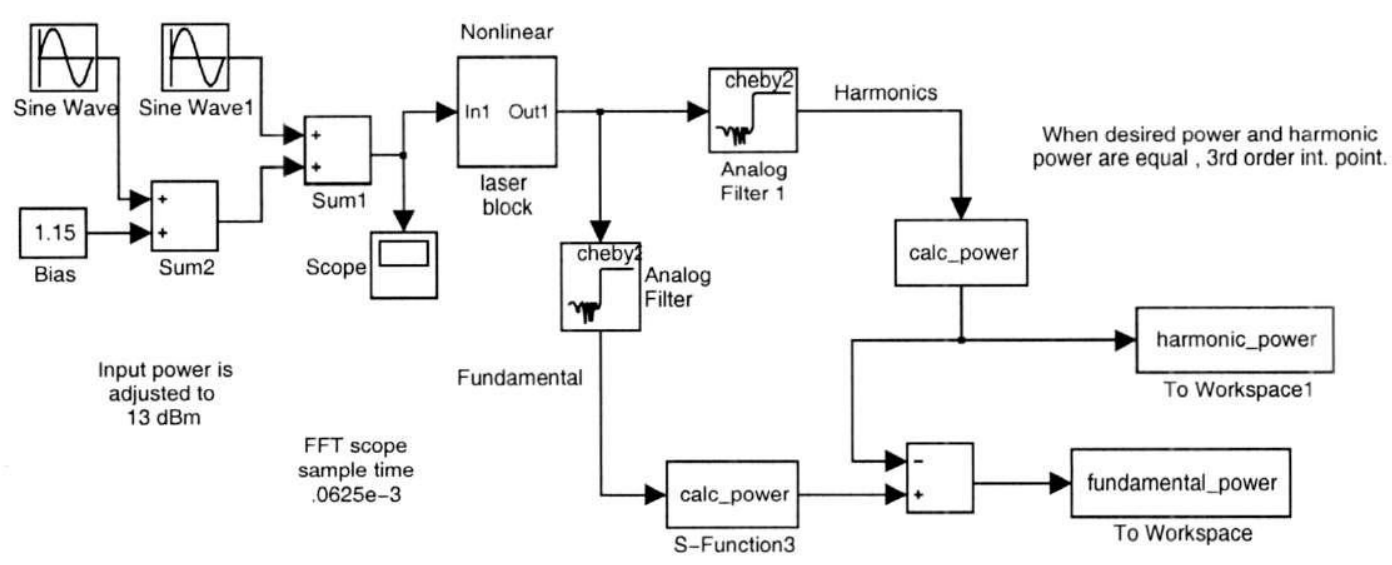

Simulation Model to Derive an Expression for the Laser Transfer Function

Figure 2.4: Simulation model to identify the nonlinear transfer function of the laser diode

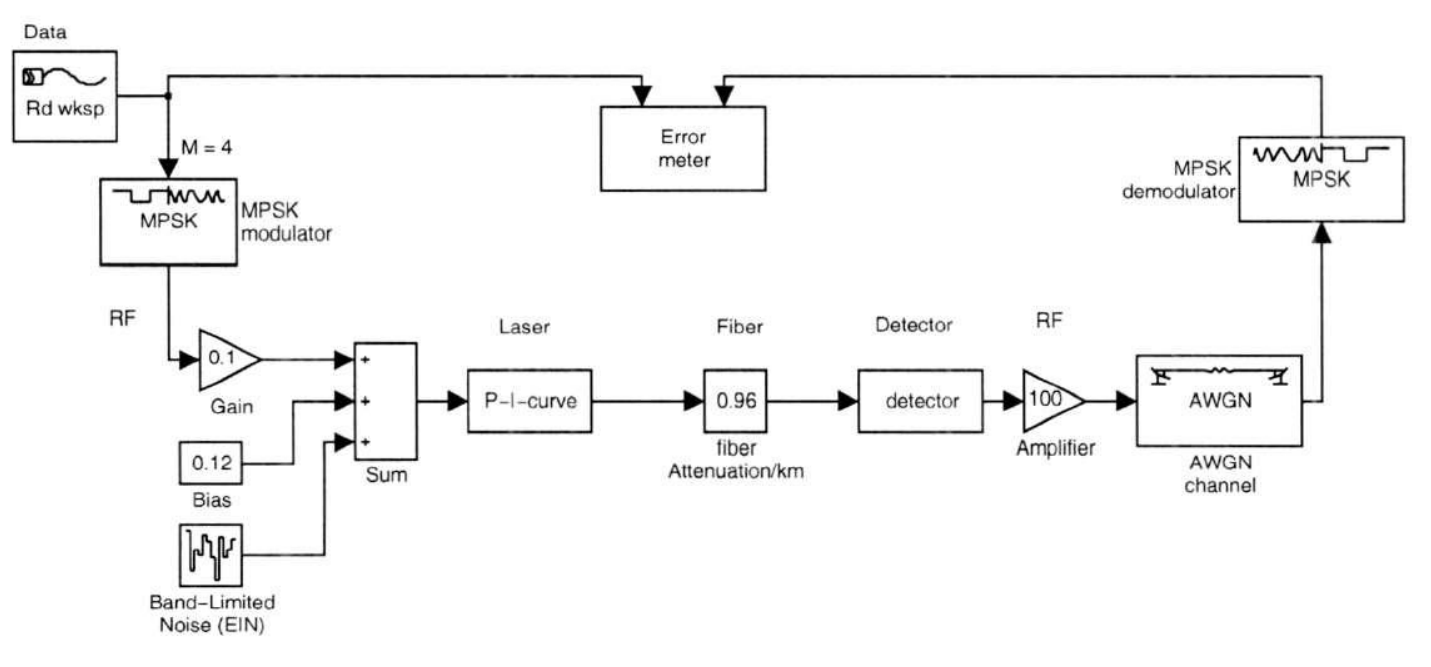

Simulation Model - Basic Optical / Wireless Link

Figure 2.5: Simulation model of the ROF link plus AWGN wireless channel

scaling factor $K=1 \times 10^{6}$. This reduces unnecessary small simulation steps. The scaled down $f_{c}$ is $1.8 \mathrm{GHz} / 10^{6}=1.8 \mathrm{kHz}$. All other frequency and time axes have been scaled accordingly. The M-ary random data is generated and modulated with MPSK modulator at $1.8 \mathrm{GHz}$. When $\mathrm{M}=4$, this represents a QPSK modulator. The power is scaled down by 0.1 in order to meet the laser input dynamic range. 


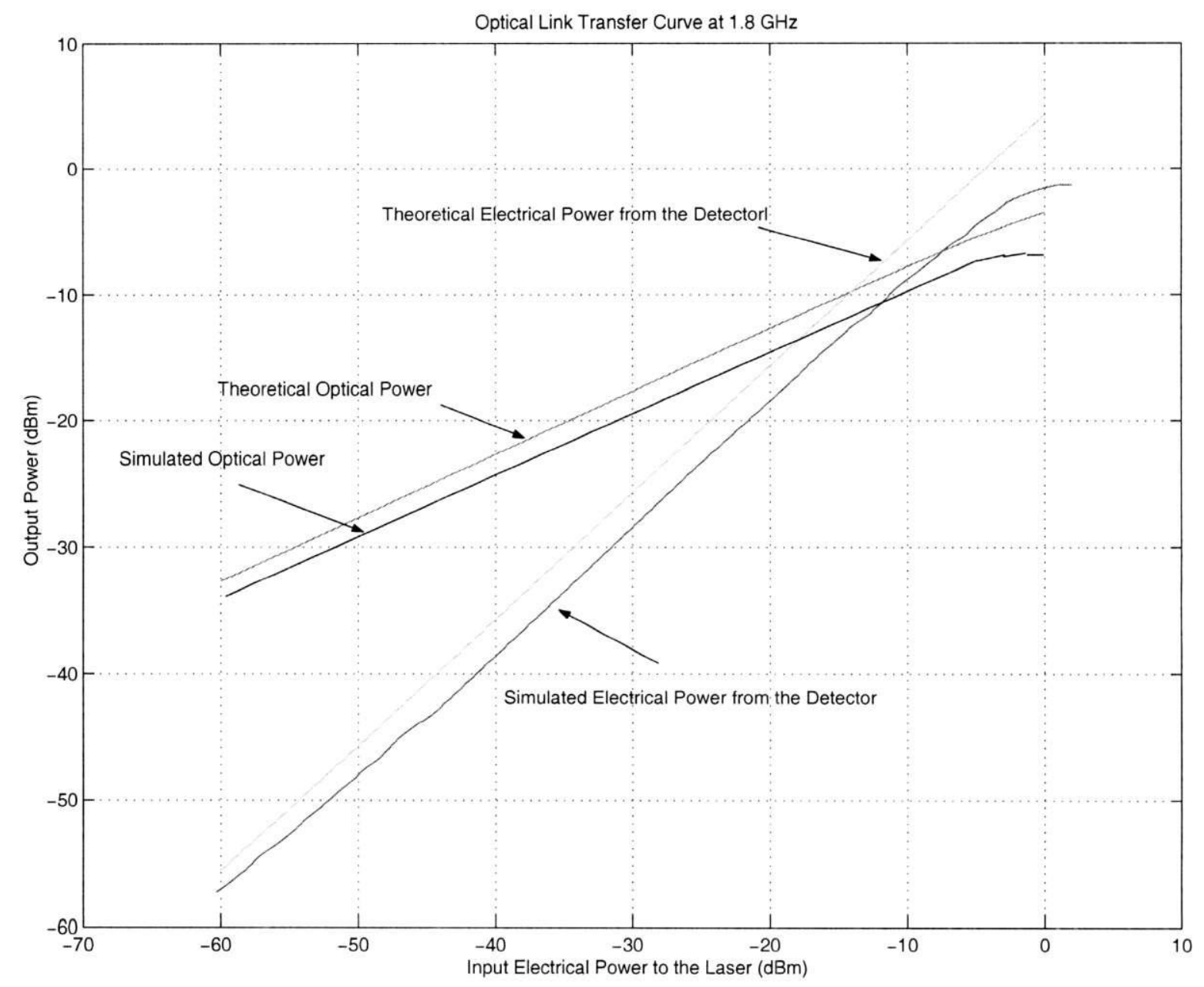

Figure 2.6: Output optical power from the laser as well as the electrical power from the detector

A DC bias and noise (EIN) are added. Parameters of the simulation model closely resembled the parameters of the prototype link to be realistic.

\subsubsection{Optical Transmitter}

For the PFT-1700 transmitter module, from manufacturer's specifications, modulation gain $G_{m}=0.12 \mathrm{~mW} / \mathrm{mA}$ (min), input one-dB compression point is $1 \mathrm{dBm}$ (min) and input third order intercept (TOI) is $13 \mathrm{dBm}$ (min). The task is to find an expression that reflects these parameters. A simulation model shown in Fig. 2.4) is used iteratively to determine this expression. Two sinusoid signals are used to create the third order intermodulation product and the TOI point is where this power 


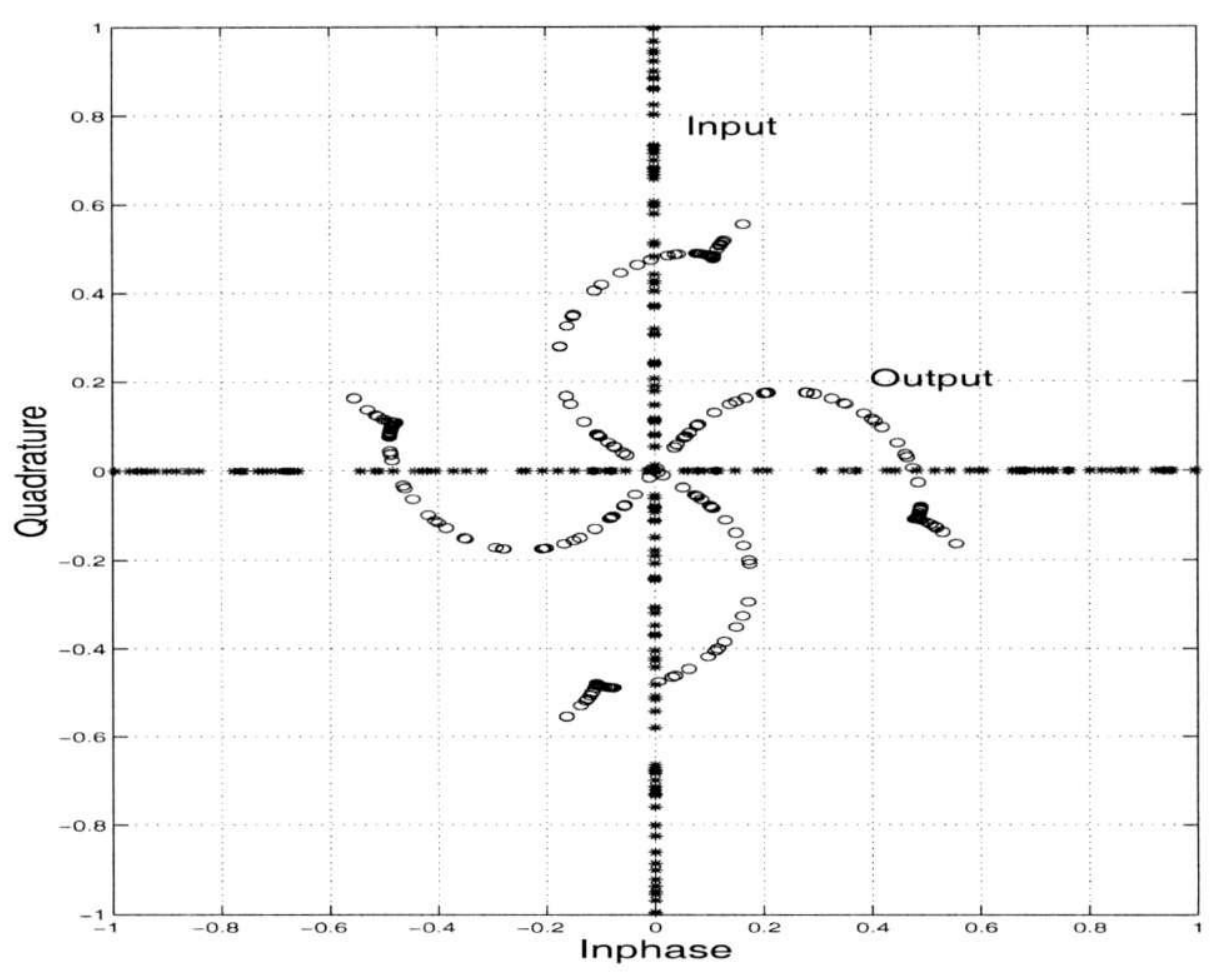

Figure 2.7: Output QPSK constellation with of a single user

is equal to the power of the fundamental tone. Using this approach, the following expression for power transfer curve is obtained for a 50 -ohm system.

$$
P_{\text {out }}=P_{\text {in }} / 2-2.703-\exp \left(0.155\left(P_{\text {in }}-1\right)\right) \quad P_{\text {in }}<13 \mathrm{dBm}
$$

Here, -2.703 is the laser conversion loss equal to $10 \log _{10}(0.12 \times 1000 / 50)$. At low power the exponential term is insignificant while at high power it introduces saturation effect and ensures that at $13 \mathrm{dBm}$ the TOI is met.

\subsubsection{The Fiber-Wireless Link}

Fig. 2.5 shows the simulation block diagram for the fiber wireless link. This simulation model is mainly used to provide a preliminary idea of the power and noise levels at various portions of the link. Virtually, there is no dispersion in the fiber at $1.8 \mathrm{GHz}$, as we have shown in section 2.2. Therefore, only an optical power attenuation of $0.5 \mathrm{~dB} / \mathrm{km}$ is applied to the fiber. The detector with a linear optical 


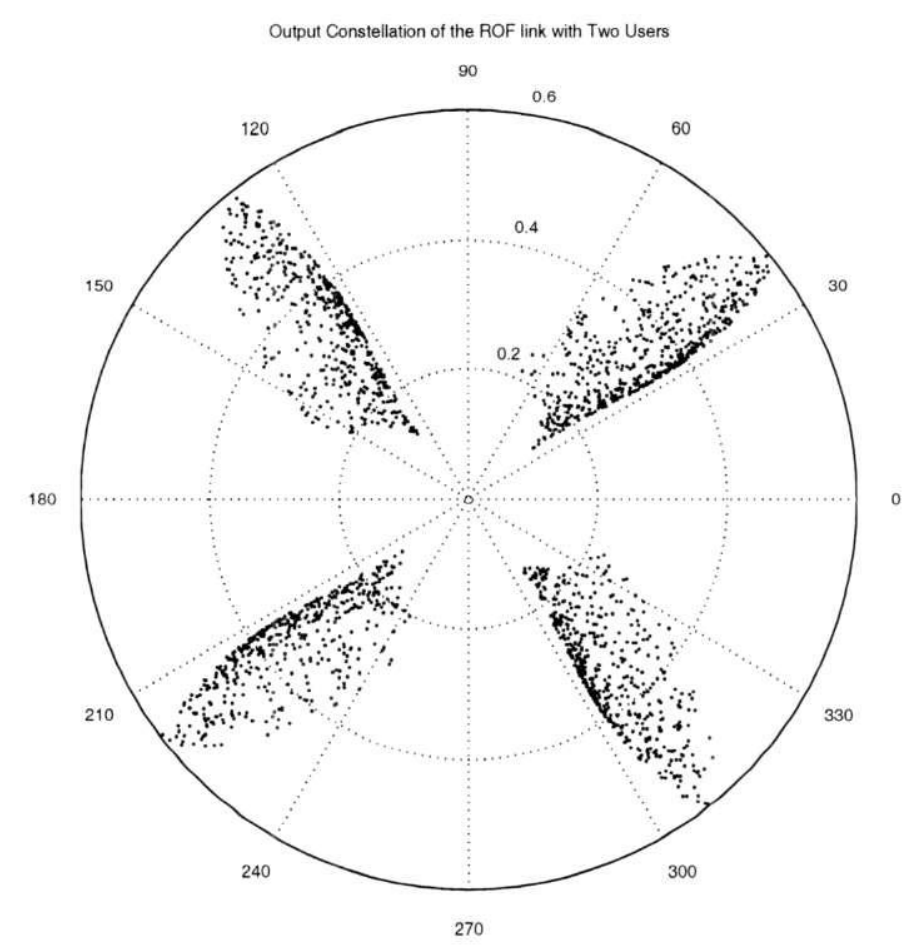

Figure 2.8: Output QPSK constellation with two users

responsivity of $0.75 \mathrm{~mA} / \mathrm{mW}$ with adequate bandwidth is used to detect the signal. Detector shot and thermal noise equivalents are added at the optical receiver.

Fig. 2.6 shows both simulated and theoretical optical as well as the electrical power output of the ROF link. The optical output is from the laser into the fiber while the electrical output is from the detector. A post amplifier is used to make the overall link gain approximately $+5 \mathrm{~dB}$. If there is no laser saturation then the predicted curves are linear. Note that when the input power changes by $10 \mathrm{~dB}$, the optical power changes by $20 \mathrm{~dB}$. This is because the optical power is proportional to injected current, while the input power is proportional to current squared. This is the reason why optical domain losses appear twice in the electrical domain.

Next, a simple AWGN channel is used to represent the wireless channel. QPSK demodulation is done after the wireless channel and BER is computed. Fig. 2.7 shows the output QPSK constellation of a single user. The amplitude of the input symbols varied from 0 to 1 while the phase is kept constant. However, in the received output constellation, the amplitude is compressed while, the phase is distorted as 
seen in the figure. Fig. 2.8 shows the output QPSK constellation of a single user when signals from two different users are transmitted through a single ROF link. The RF power of both users varied, keeping the total power less than $0 \mathrm{dBm}$. Since, the phase shift is a function of the total RF power the received constellation is quite dispersed. The simulation was run at $1.8 \mathrm{GHz}$.

In this chapter, the ROF link has been analyzed both theoretically and by simulation. In chapter 3 , experimental observations on a prototype ROF link are described. Conclusions from all these three types of investigations are given at the end of Chapter 3 to provide a complete picture. 


\section{Chapter 3}

\section{Empirical Characterization}

In the last Chapter, we theoretically evaluated the basic characteristics of an ROF link. In this Chapter, we verify that evaluation by performing hands on measurements on prototype ROF links. We used two ROF links with identical specifications. All these measurements were done at TRLabs, Calgary.

The prototype optical transmitter (PFT1700) consists of a directly modulated, InGaAsP distributed feedback (DFB) laser. The optical receiver module consists of a PIN diode photo detector. These are connected with a 2.2-km long, 9/125 single mode fiber. FC/APC type connectors are used to reduce the back reflection. The receiver has a transimpedance amplifier and a post amplifier with a total gain of $32 \mathrm{~dB}$. Therefore, the total link gain is approximately unity at short fiber lengths. Both the low impedance laser and the detector are reactively matched to a 50-ohm system giving a flat bandpass response from 1.7 to $2.2 \mathrm{GHz}$ and high attenuation elsewhere. The technical specifications of the transmitter and the receiver are given in tables 3.1 and 3.2 .

Extensive measurements are done to characterize the ROF link. All these are RF measurements taken either at the output of the detector or at the input of the laser unless otherwise specified. The power means the RF power into the laser and not the optical power in the fiber. 


\begin{tabular}{|c|c|}
\hline Frequency of Operation & 1.7 to $2.2 \mathrm{GHz}$ \\
\hline Wavelength & $1310+/-10 \mathrm{~nm}$ \\
\hline O/E conversion factor & $0.12 \mathrm{~mW} / \mathrm{mA}(\mathrm{min})$. \\
\hline Frequency Response Flatness & $+/-0.5 \mathrm{~dB}$ \\
\hline Input 1 dB compression & $1 \mathrm{dBm}$ \\
\hline Input VSWR (50 ohms) & $2: 1$ \\
\hline Input Third Order Intercept (TOI) & $13 \mathrm{dBm}(\min )$. \\
\hline Equivalent Input Noise & $-125 \mathrm{dBm} / \mathrm{Hz}(\max )$. \\
\hline
\end{tabular}

Table 3.1: Key specifications of the optical transmitter PFT 1700

\begin{tabular}{|c|c|}
\hline Frequency of Operation & 1.7 to $2.2 \mathrm{GHz}$ \\
\hline Wavelength & 1000 to $1600 \mathrm{~nm}$ \\
\hline DC Optical Responsivity & $0.75 \mathrm{~mA} / \mathrm{mW}(\mathrm{min})$. \\
\hline Frequency Response Flatness & $+/-0.6 \mathrm{~dB}$ \\
\hline Optical Return Loss & $45 \mathrm{~dB}$ \\
\hline Output VSWR (50 ohms) & $2: 1$ \\
\hline Fiber & Single Mode $(9 / 125)$ \\
\hline Max. Optical Input Power & $2 \mathrm{~mW} \mathrm{CW}$ \\
\hline
\end{tabular}

Table 3.2: Key specifications of the optical receiver PFR 1701

\subsection{Basic Nonlinearity Measurements}

Two types of measurements are performed. Basic measurements are done to obtain basic nonlinear characteristics of the ROF link using standard RF test equipment such as signal generators, spectrum analyzers and network analyzers. These are discussed in this section. There are some additional measurements done using the impulse response identification system (IRIS) with realistic modulated signals. Those are described in section 3.2 .

\subsubsection{Effects of Fiber Dispersion}

The first attempt is finding out the effect of fiber dispersion on a single RF tone. To study this, an unmodulated carrier at $1.8 \mathrm{GHz}$ is transmitted through the ROF link with $-30 \mathrm{dBm}$ RF power. The input spectrum and output spectrum of this carrier are plotted in Fig. 3.1. It can be seen, even up to $50 \mathrm{~dB}$ below carrier level, there is no visible dispersion or distortion on a single RF tone due to ROF link, when the laser operates in the linear region. 

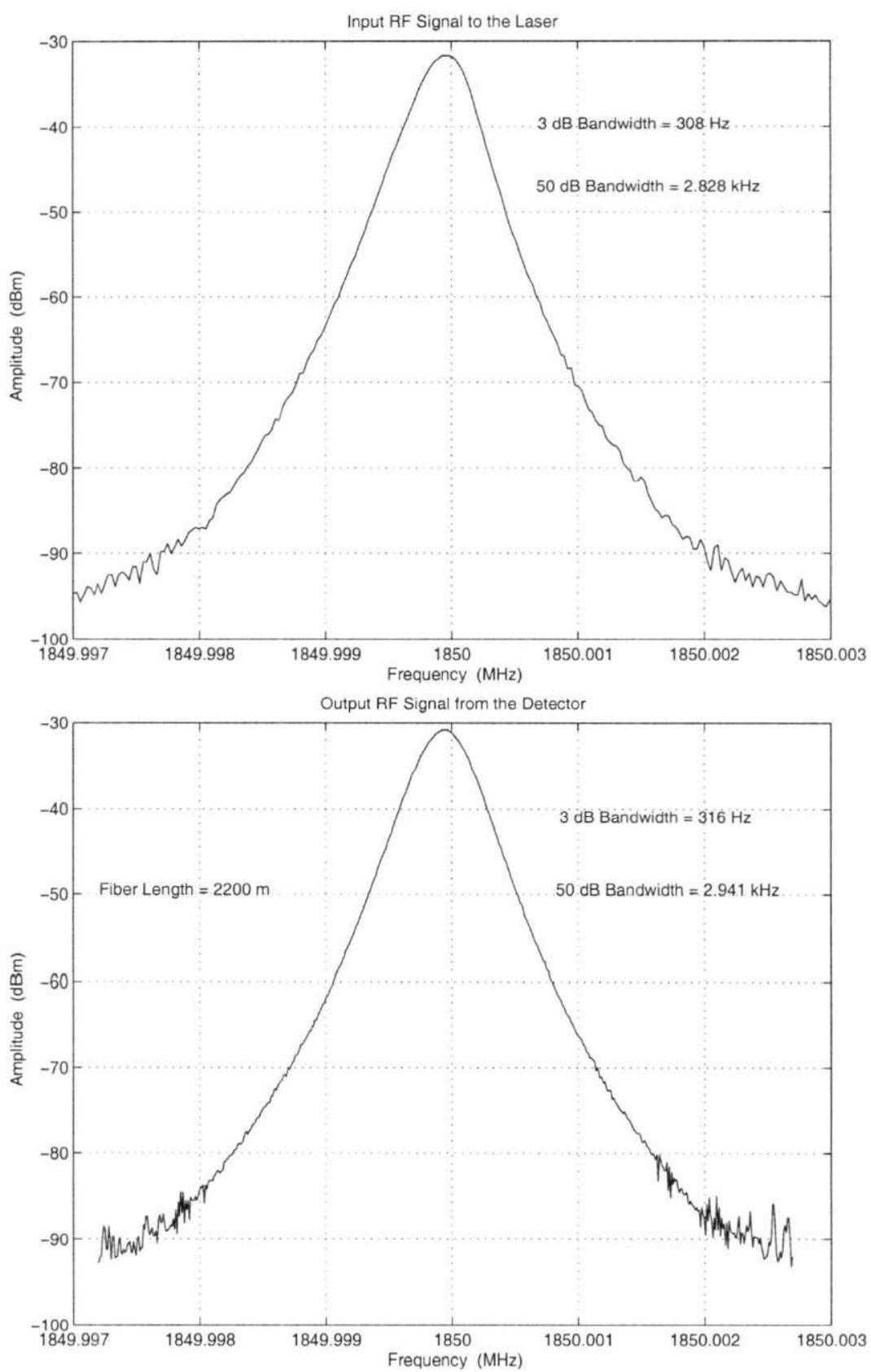

Figure 3.1: A single tone at the input and output of the ROF Link

\subsubsection{Frequency Response}

The amplitude response of the ROF links is shown in Fig. 3.2. This is measured at two different input power levels: at $-20 \mathrm{dBm}$ and at $+5 \mathrm{dBm}$. Within the linear range (that is, with $-20 \mathrm{dBm}$ input power), the amplitude response is almost 


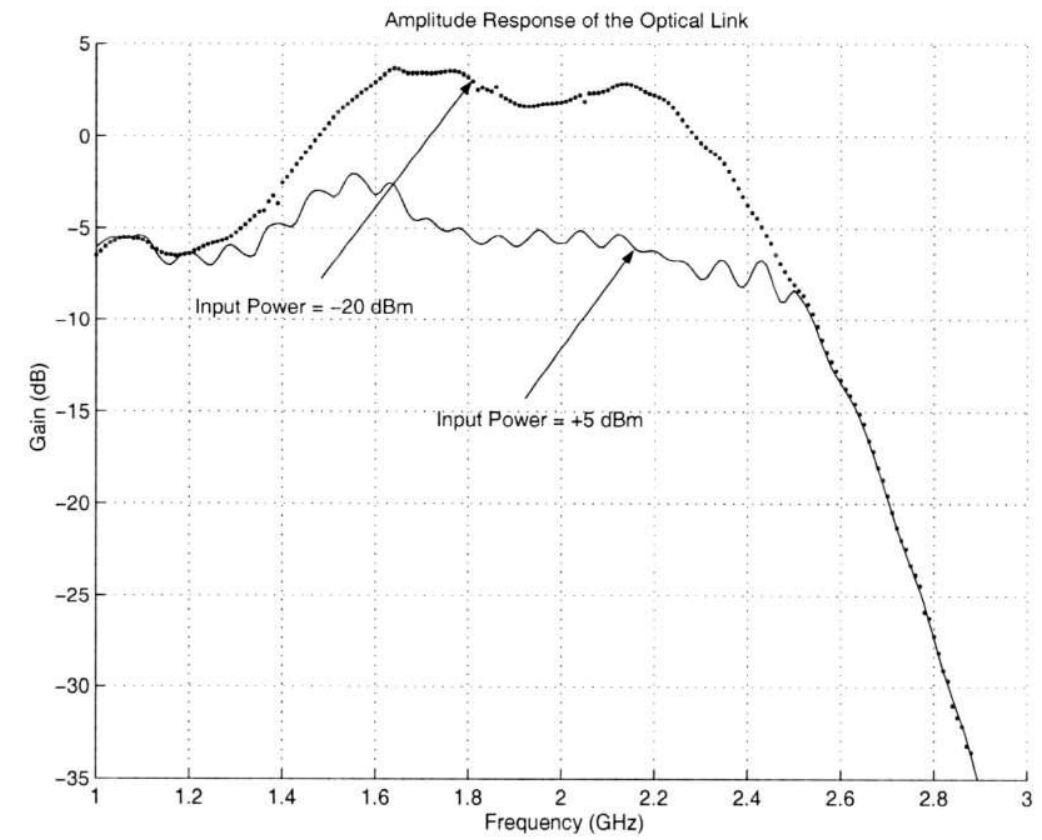

Figure 3.2: Amplitude response of the ROF link with two different input powers

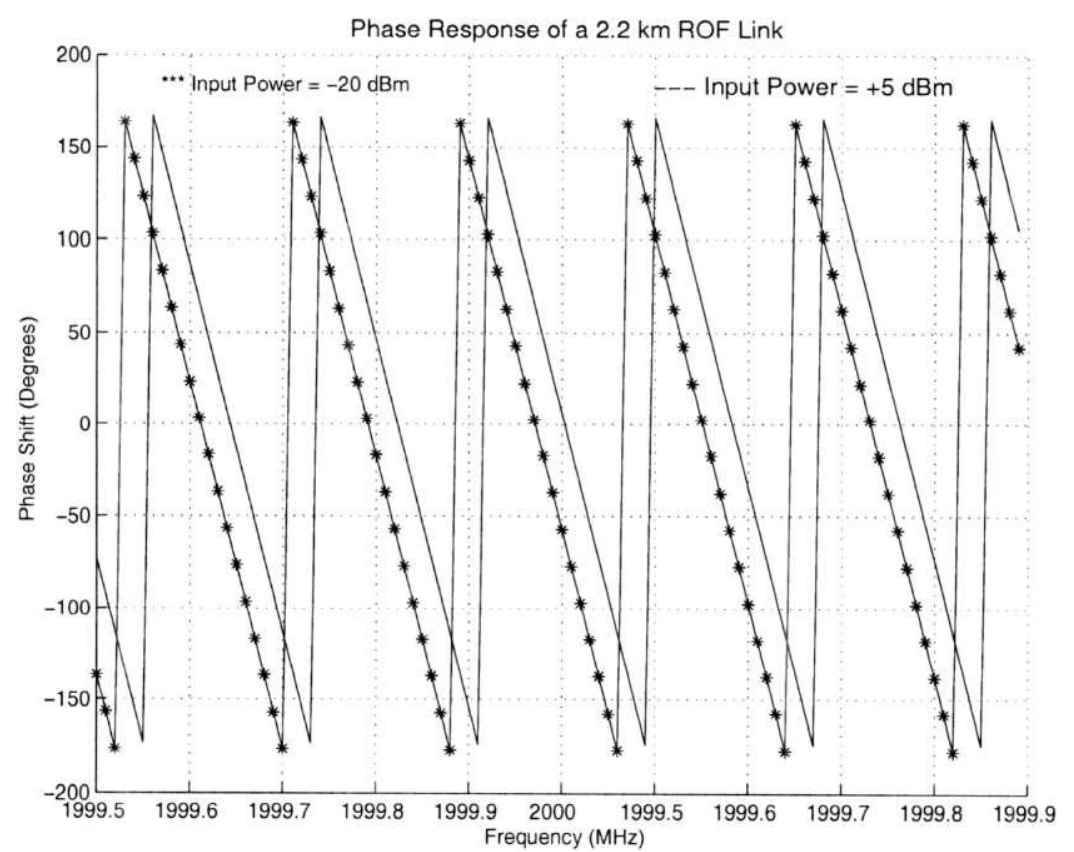

Figure 3.3: Phase response of the ROF link with two different input powers 


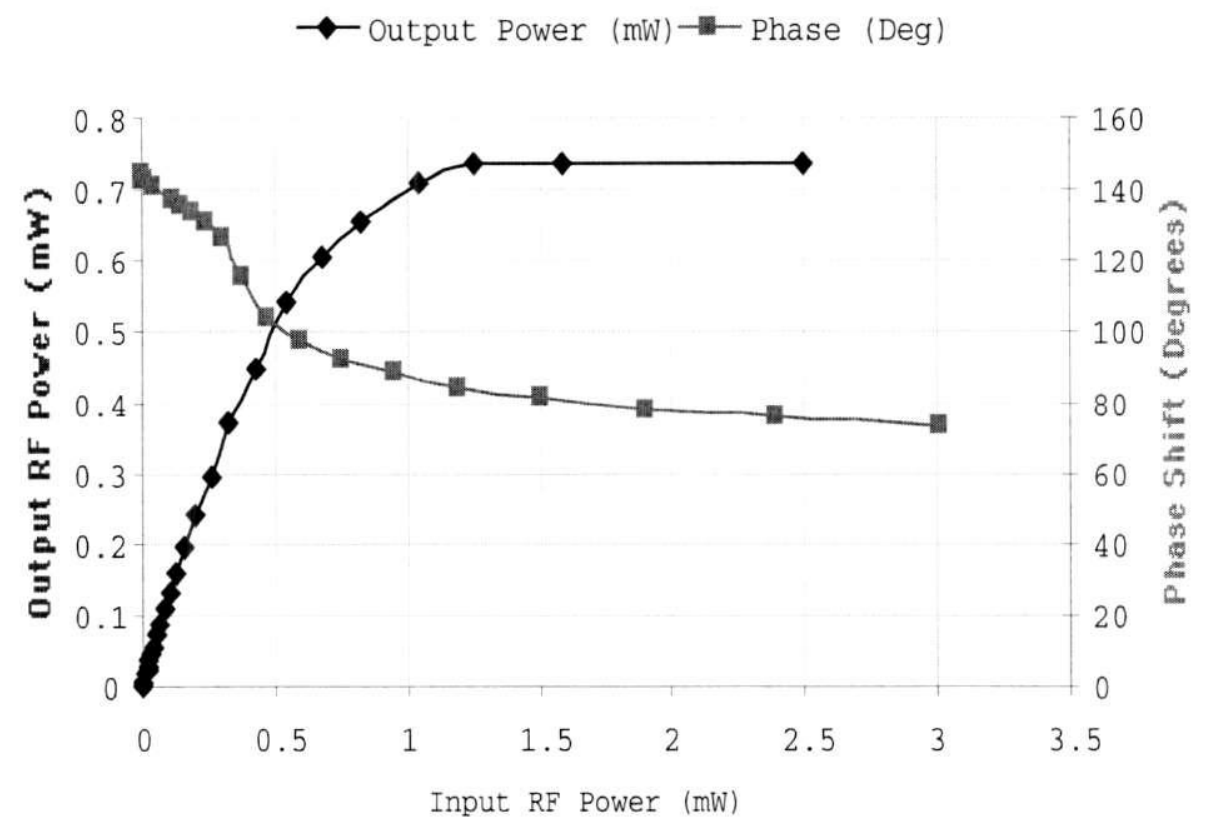

Figure 3.4: AM-AM and AM-PM characteristics of the ROF link

constant from 1.7 to $2.2 \mathrm{GHz}$. Measured 3-dB bandwidth is $0.76 \mathrm{GHz}$ (from 1.53 to $2.29 \mathrm{GHz})$. The attenuation is high out of this band due to the reactive matching. Note that, the amplitude response depends on the input RF power. When the input power level is $+5 \mathrm{dBm}$, the amplitude response shows much lower gain and small variations with frequency.

The HP network analyzer (HP8510) is used to obtain this response. Note that the network analyzer transmits a single tone at a certain power level and compares it with the power of the received tone at that particular frequency. Therefore, it is unable to detect the power in the harmonics, which would have been significant at $+5 \mathrm{dBm}$. However, this is characterized better in the two-tone measurement test that comes next.

The phase response (Fig. 3.3) is quite linear with respect to frequency, exhibiting a constant slope. This slope is commonly referred as the group delay. The group delay depends on the fiber length. For a fiber length of $2.2 \mathrm{~km}$, measured slope is $2 \pi / 90 \mathrm{kHz}$. Calculated group delay from this slope is $1 /\left(90 \times 10^{3}\right)=11.11 \mu \mathrm{s}$. This 


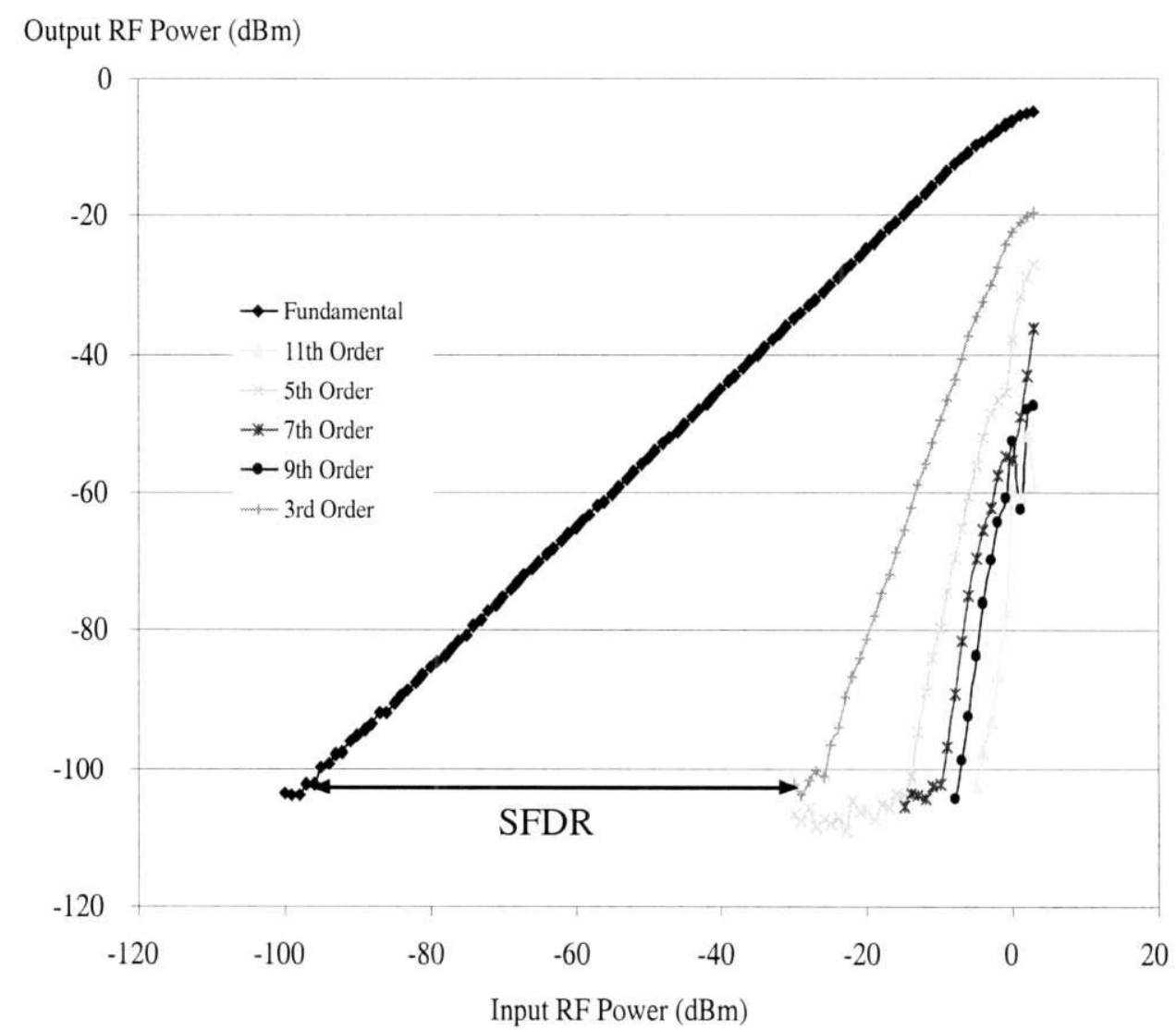

Figure 3.5: Two-tone test results of the ROF link

agrees with the theoretical calculation as follows. The velocity of light in a single mode fiber is given by the speed of light in vacuum $\left(3 \times 10^{8} \mathrm{~m} / \mathrm{s}\right)$ divided by the core refractive index (1.51). Neglecting any variations in the core refractive index, the time taken for the light to travel $2200 \mathrm{~m}$ is, $2200 \times 1.51 /\left(3 \times 10^{8}\right)$ which is 11.10 $\mu \mathrm{s}$.

However, the variation of phase response with respect to RF power is not constant suggesting AM-PM distortion. This point is reviewed in section 3.1.3.

\subsubsection{AM-AM and AM-PM Measurements}

These measurements are made to study the amplitude dependent amplitude (AM$\mathrm{AM}$ ) and amplitude dependent phase (AM-PM) distortions of the ROF link. These measurements are key in nonlinearity characterization. HP network analyzer HP8510 


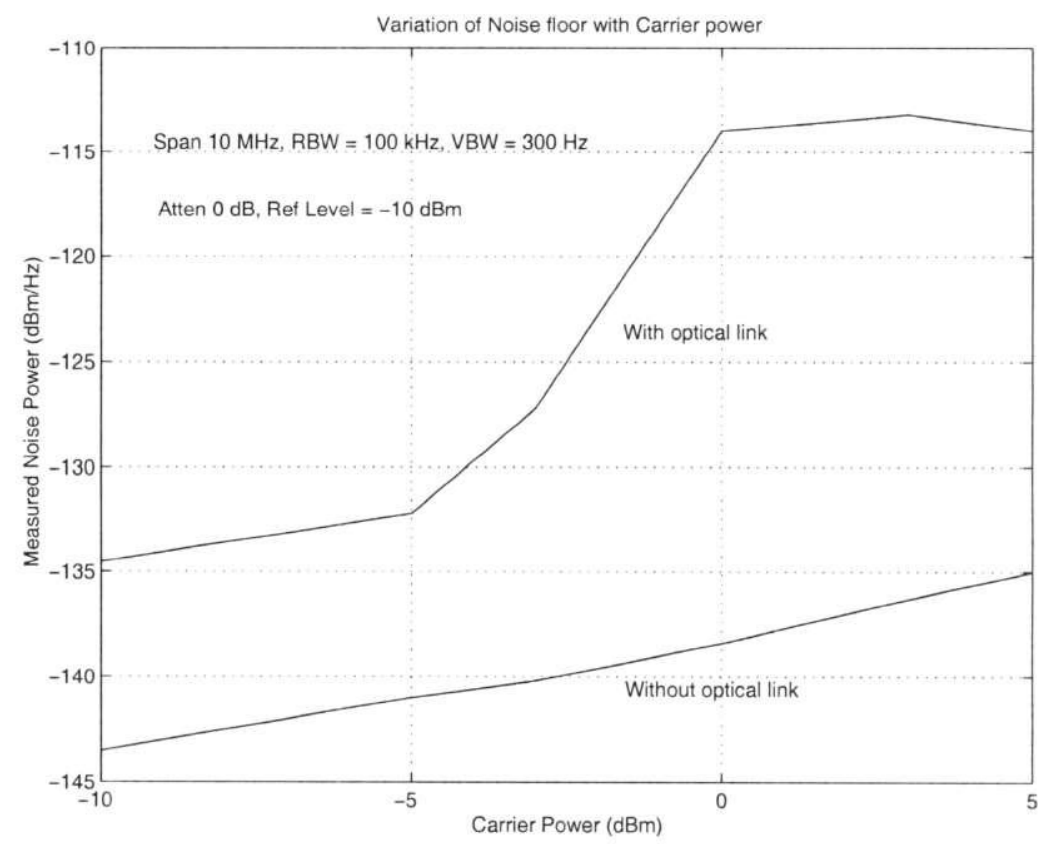

Figure 3.6: Noise floor measurements of the ROF link

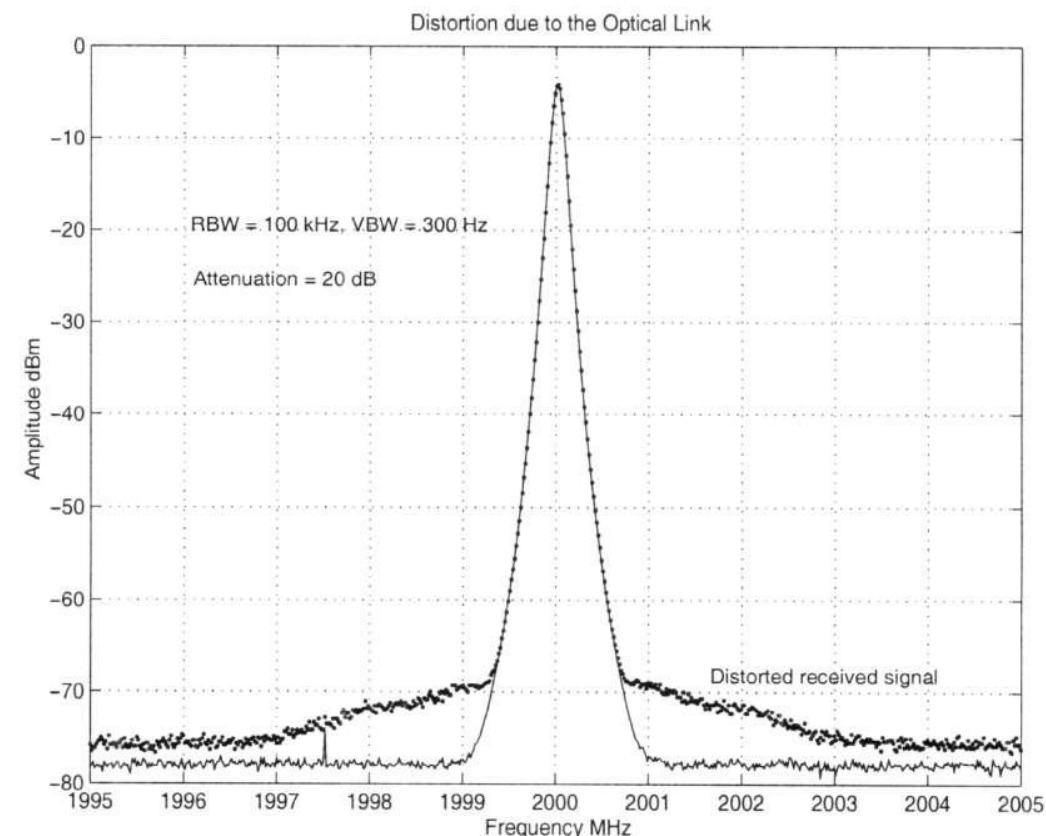

Figure 3.7: Frequency dependent noise floor of the ROF link 
is used for these measurements and the measurements are done at $1.8 \mathrm{GHz}$. The input RF power to the laser is changed in small steps and each time the output RF power from the detectors as well as the phase shift between the input and output is recorded. Note that, the $32 \mathrm{~dB} \mathrm{RF}$ amplifier at the optical receiver compensates for the $\mathrm{O} / \mathrm{E}$ and $\mathrm{E} / \mathrm{O}$ conversion losses so that, with $2.2 \mathrm{~km}$ long fiber the overall gain of the link is approximately one. From Fig. 3.4, the amplitude gain of the link is approximately constant up to about $0.7 \mathrm{~mW}$ then, it starts to saturate. The output amplitude is fully saturated after $1.2 \mathrm{~mW}$ input power. On the other hand, the phase shift of the link is quite nonlinear from the beginning. This is a major concern with phase-modulated schemes.

\subsubsection{Two-Tone Test}

Two-tone test results are important in characterizing a bandpass nonlinear device. These results are shown in Fig. 3.5. Note that the one- $\mathrm{dB}$ compression point is at -3 $\mathrm{dBm}$. This is different from the manufacturer's specifications because there are two tones involved here. The noise floor is at $-100 \mathrm{dBm}$. However, we should note that the noise floor is a function of the video bandwidth used in the spectrum analyzer, which is $100 \mathrm{kHz}$ in this case. It also decreases with the averaging function available with most of the spectrum analyzers. The noise floor generally increases with the post-detection bandwidth of a particular modulation scheme. This is especially high with spread spectrum systems. For example in GSM systems, the post-detection bandwidth is $200 \mathrm{KHz}$ while in IS-95 it is $1.25 \mathrm{MHz}$.

From Fig. 3.5, inter-modulation products start to appear at about $-25 \mathrm{dBm}$. This is much lower than the one- $\mathrm{dB}$ compression point. Therefore, the spurious free dynamic range (SFDR) is approximately $75 \mathrm{dBm}(-100-(-25)=75)$.

Furthermore, we observed the following characteristics by performing additional measurements. These graphs are not shown considering the space constraint. Here, if $f_{1}<f_{2}$, then we refer $2 f_{2}-f_{1}$ as the positive intermodulation product because, $2 f_{2}-f_{1}>f_{1}$. Similarly, we refer $2 f_{1}-f_{2}$ as the negative intermodulation product because, $2 f_{1}-f_{2}<f_{1}$. These are defined assuming $f_{1}$ as our reference frequency.

- Both positive and negative third order inter-modulation products have the 
same characteristics. This is true for other odd order inter-modulation products (like the 5 th and 7 th order) too.

- Power of the inter-modulation products do not depend on the frequency separation of the fundamental tones. This is verified by varying the frequency separation and then recording the inter-modulation power.

- The inter-modulation product does not depend on the frequencies of the input tones while the separation between them is kept the same. This is verified by varying the frequencies while keeping their separation constant and then recording the inter-modulation power.

From these observations, we conclude that the nonlinear distortion is not a function of radio frequency at least within our pass band. This is in agreement with previously reported results at [9] and [33].

\subsubsection{Noise Measurements}

The characteristics of the noise floor within the pass band are shown in Fig. 3.6. These are measured using a HP8563 spectrum analyzer and HP8648D RF generator at $2 \mathrm{GHz}$. It clearly shows that the noise floor of the optical link suddenly increases as the signal level becomes close to the saturation level of the ROF link $(-3 \mathrm{dBm})$. Probably the sudden increment is because of the clipping at the laser. Note that, even without the optical link, the noise floor linearly increases. This is due to the inherent limitation of the spectrum analyzer.

A strange phenomenon is observed in the noise floor of the ROF link. This is shown in Fig. 3.7, where a clean single RF carrier, and the same carrier after transmission through the ROF link are plotted on the same window. An increment in the noise floor close to center frequency is clearly visible in the figure. This may not be due to clipping because clipping gives a wideband noise spectrum. This frequency dependent increment in the noise floor happens, when the input RF power is near the saturation level $(-3 \mathrm{dBm})$. When the carrier power is further increased, this bump in the noise floor further widens and covers the whole spectrum. At low carrier powers (less than $-10 \mathrm{dBm}$ ), the noise floor is constant. 


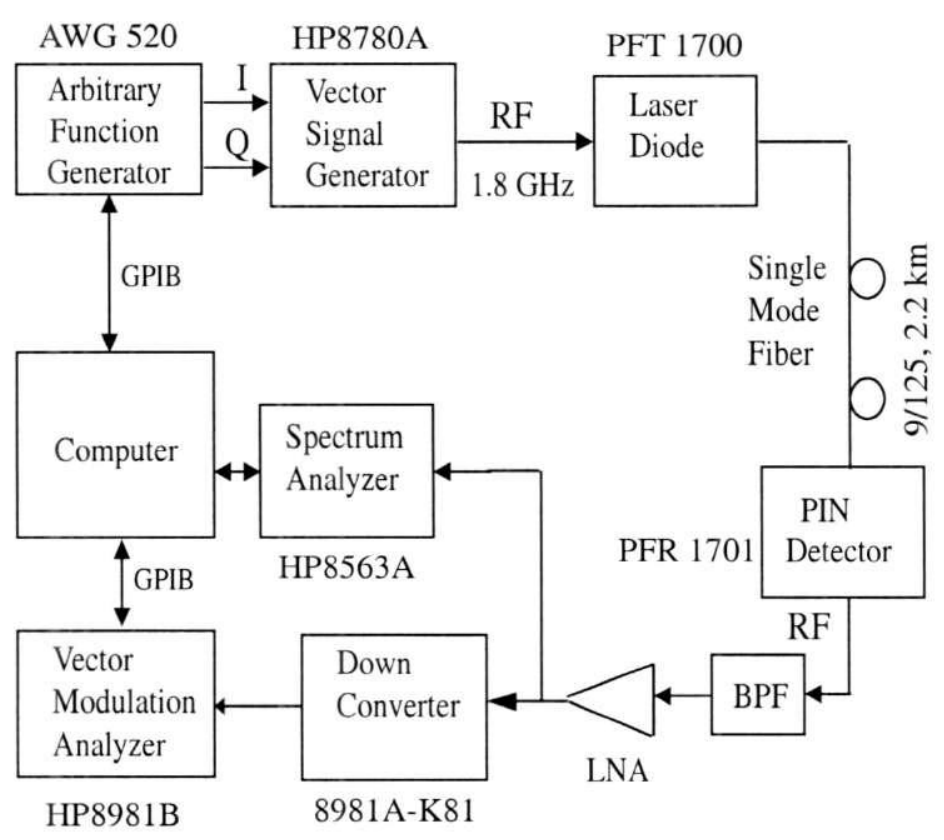

Figure 3.8: Experimental setup for dynamic measurements

These observations suggest that close to saturation the phase noise of the ROF link increases as in Fig. 3.7. At further high RF power, the clipping starts to dominate giving a wideband noise spectrum.

\subsection{Dynamic Measurements}

When a dynamically fluctuating modulated RF signal is transmitted through the ROF link, the distortions may be different from those observed during static or tone measurements. This is because now, the dynamic nonlinear characteristics of the laser diode would come into play, which might give a different behavior. These measurements give a more realistic evaluation of a real wireless system where, the signal dynamically fluctuates. Fig. 3.8 shows the arrangement used to obtain most of these measurements.

\subsubsection{Constellation Measurements}

The constellation of vector-modulated symbols plays a key role in the BER performance. However, the constellations are often distorted due to nonlinear channels. 

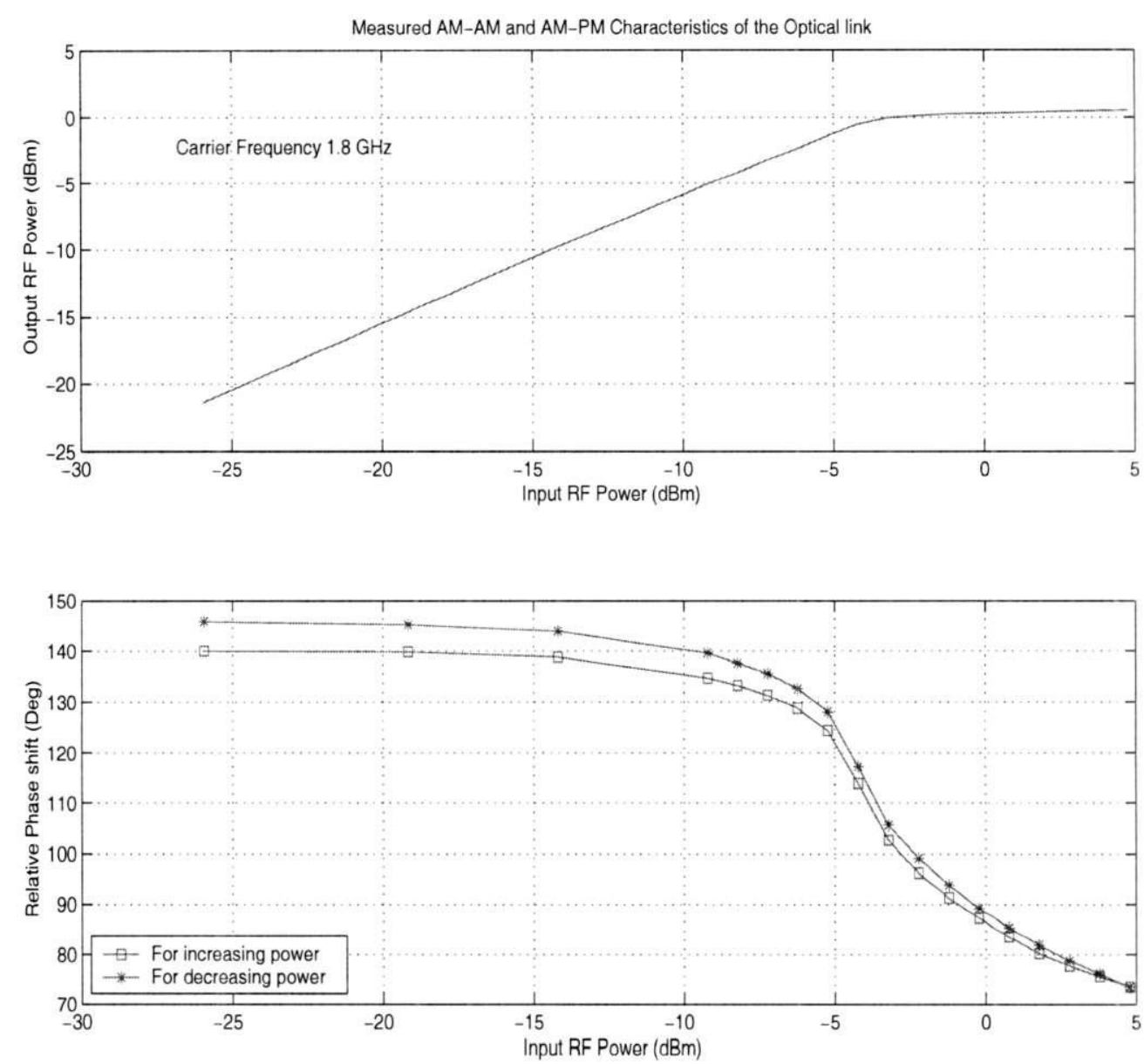

Figure 3.9: Amplitude and phase distortions of vector modulated symbols after transmission through the ROF link

To investigate this effect, the measurements shown in Fig. 3.9 are done. Here, a random data sequence is generated from the arbitrary waveform generator (AWG520). Then, it is modulated with the RF Signal Generator in the vector modulation mode. In the vector modulation mode, the modulating sequence is split into both inphase and quadrature components and the RF carrier is modulated in two dimensions as in QPSK. This modulated carrier is transmitted via the optical link, received, then down converted and the constellation is displayed on the vector modulation analyzer. The magnitude and phase of the received vector symbols are measured. This procedure is repeated by changing the input RF power and each time the magnitude and phase of the received vector symbols are recorded. These are shown in Fig. 3.9. From the upper window of the figure, amplitude of the received constellation 
starts to saturate at $-3 \mathrm{dBm}$. This is similar to the saturating point at the two-tone tests. The big concern here is the phase shift. The phase of the received QPSK symbols starts to change at a much lower power level $(-15 \mathrm{dBm})$. Furthermore, note that two slightly shifted phase curves are obtained depending on whether the power is increasing or decreasing. During measurements the RF power was initially -24 $\mathrm{dBm}$, then it was increased up to $+5 \mathrm{dBm}$ then again decreased to $-24 \mathrm{dBm}$. At the end, there is a residual phase error of $5^{\circ}$. This suggests a hysteresis type, long-term memory of the ROF link.

\subsubsection{Interference Measurements}

These measurements are taken to analyze the interference between two users with separate RF carriers, who share the same ROF link. Two sets of measurements are done to understand the effect of frequency separation between the users as well as the effect of RF power level of each user.

\subsubsection{Effect of Interfering Power}

First two independent random data sequences are generated, each at one Mega symbols per second. These are QPSK modulated on to the RF carrier. The carrier frequencies are $1.8 \mathrm{GHz}$ for the desired user and $1.86 \mathrm{GHz}$ for the interfering user. Both these carriers are combined with a power combiner and transmitted through the ROF link simultaneously. We found that these frequencies had to be separated by at least $45 \mathrm{MHz}$, due to the limitation of the IRIS system. (The vector modulation analyzer HP8981B can't distinguish between carriers closer than 45 $\mathrm{MHz})$.

The interfering power is changed from -10 to $+10 \mathrm{dBm}$ and, each time we decreased the power of the desired user until its constellation is no longer distinguishable. This minimum power of the desired user is recorded. We refer this power as the 'minimum detectable power' (MDP). When the power is less than this, the BER goes very high. Hence, this is a robust way of measuring the BER threshold. This procedure is repeated with and without the optical link and the observations are recorded in Fig. 3.10. The same experiment is repeated with the interfering frequency $2.1 \mathrm{GHz}$ (frequency separation $=300 \mathrm{MHz}$ ) and plotted on the same 
graph.

From Fig. 3.10, the following points can be observed:

1. Consider the points without the optical link. When the carrier separation is $300 \mathrm{MHz}$ or more, there is almost no interference due to the RF combiner, co-axial cables or other imperfect devices in the measurement set-up. This is true for whatever the interfering power may be. However, when this combined signal is transmitted via the optical link, the interfering signal power significantly affects the minimum detectable power (MDP) although both carriers are far away.

2. When the carrier separation is $60 \mathrm{MHz}$, still the same argument holds. However, the IRIS system with its imperfect connectors introduces some interference when the interfering power is at least $45 \mathrm{~dB}$ higher than the desired power.

3. When both carriers are transmitted via the ROF link, the interference depends mainly on the RF power, not on the frequency separation.

\subsubsection{Effect of Interfering Frequency}

The above experiment is repeated keeping the interference power constant at +10 $\mathrm{dBm}$ and changing the frequency separation. The observations are plotted in Fig. 3.11 , the following points can be observed from this figure:

1. When the frequency separation is less than $200 \mathrm{MHz}$, the IRIS system itself introduces interference because of the high interference power.

2. Irrespective of the frequency separation, the optical link introduces heavy interference requiring a MDP of $-15 \mathrm{dBm}$.

3. Whether the interference is a modulated signal or an unmodulated carrier it does not make much difference.

From these observations we conclude that although the optical channel has wide bandwidth, when the interfering power is high the interference is high. Thus, the power of each user through the link dominates interference irrespective of their frequency separation. 


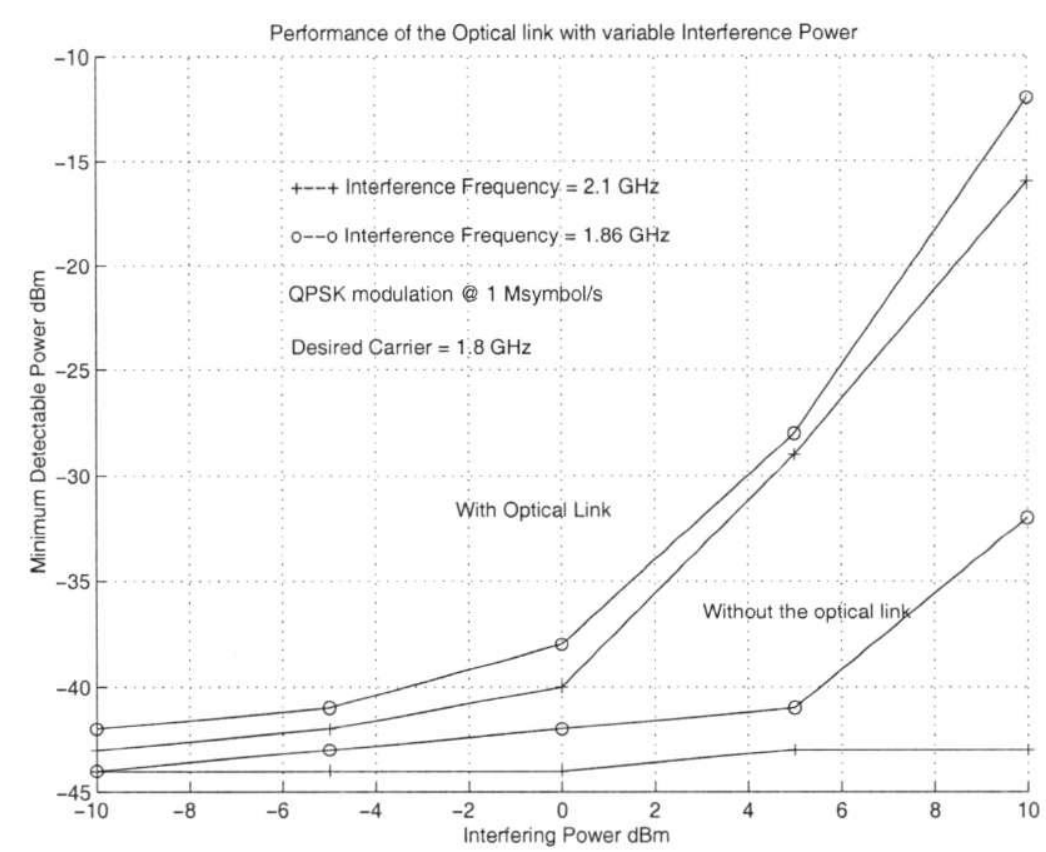

Figure 3.10: Effect of the interference power when multiuser signals are transmitted through the ROF link

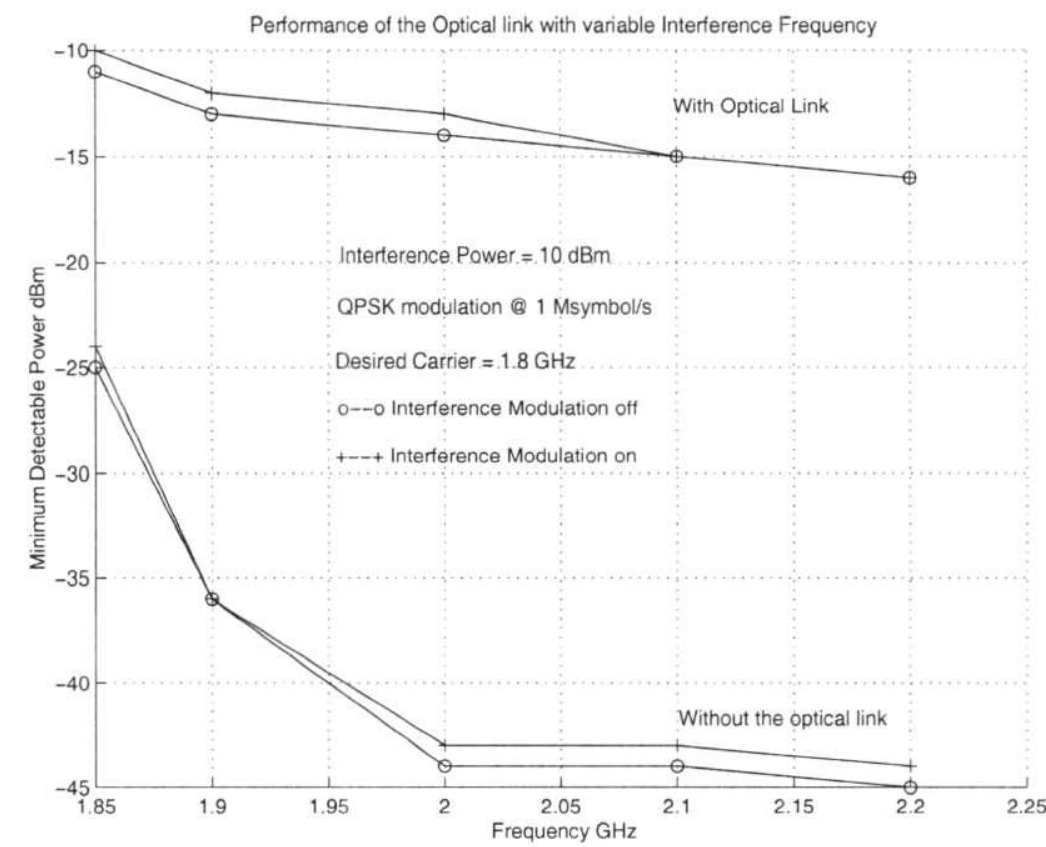

Figure 3.11: Effect of the interfering frequency when multiuser signals are transmitted through the ROF link 


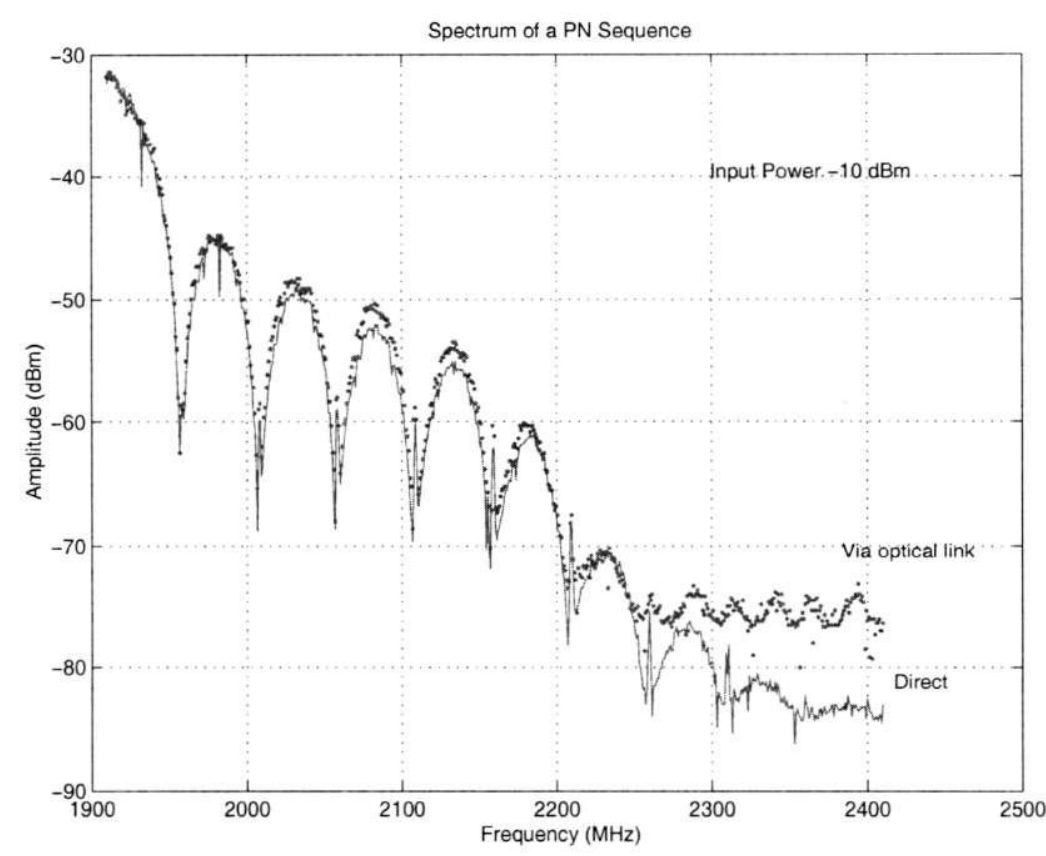

Figure 3.12: Power spectral density of a spread spectrum signal after transmission via the $\mathrm{ROF}$ link at $-10 \mathrm{dBm}$

\subsubsection{Power Density Spectrum}

These measurements are made to analyze the deformation in the power density spectrum of a spread spectrum signal through the optical link. First, 1000 bits of random data was generated and it was spread with a PN sequence of length 31 giving 31000 chips. These chips are BPSK modulated on a $1910 \mathrm{MHz}$ RF carrier without any pulse shaping. A chip time $T_{c}$ of $20 n s$ was used. This direct spread signal is transmitted via the ROF link. With this arrangement, the transmitted $\mathrm{RF}$ power is changed and at each power level, the power spectral density of the received signal is plotted. This procedure is repeated without the optical link for comparison. Both of these data are plotted in the same window in each case. These are shown from Fig. 3.12 to Fig. 3.15.

Here, the noise floor is high because of the higher bandwidth and we could detect only up to $-40 \mathrm{dBm}$. When the RF power is less than $-40 \mathrm{dBm}$, the spectrum is almost buried in the noise. This indicates that although the noise floor with single or two tones was about $-100 \mathrm{dBm}$ (see Fig. 3.5), with a real CDMA type of signal it is much higher. This significantly reduces the available dynamic range. 


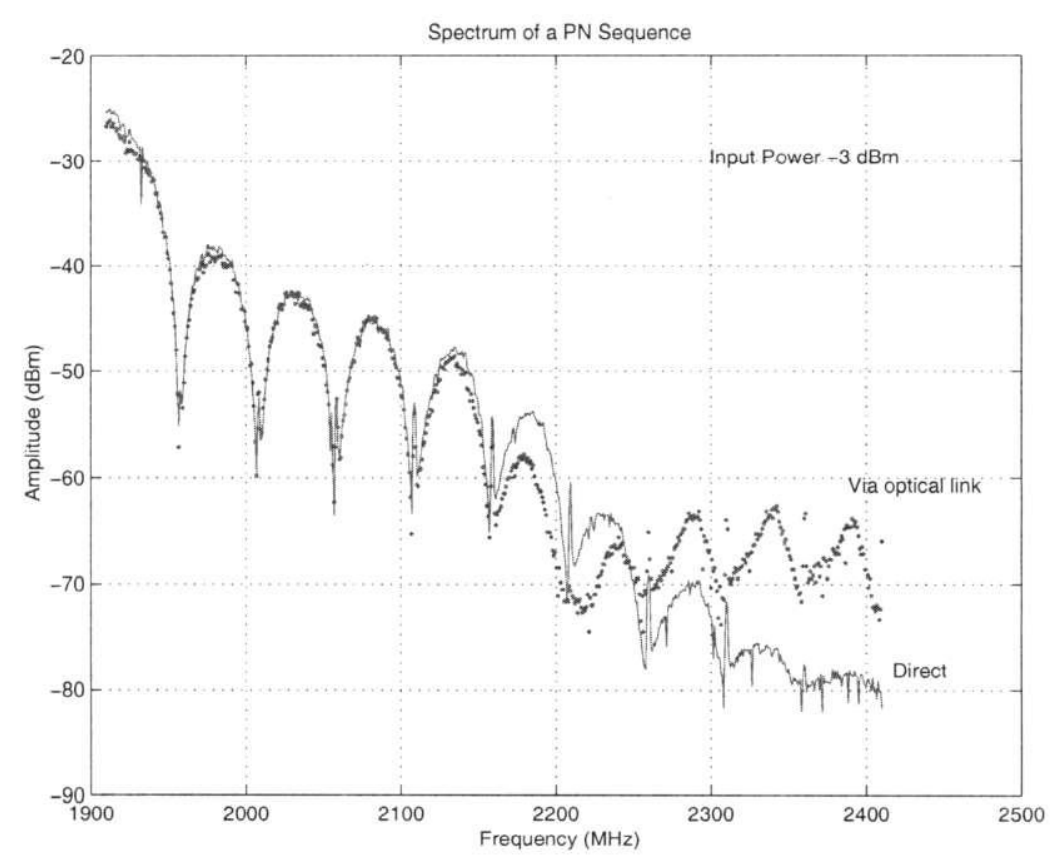

Figure 3.13: Power spectral density of a spread spectrum signal after transmission via the ROF link at $-3 \mathrm{dBm}$

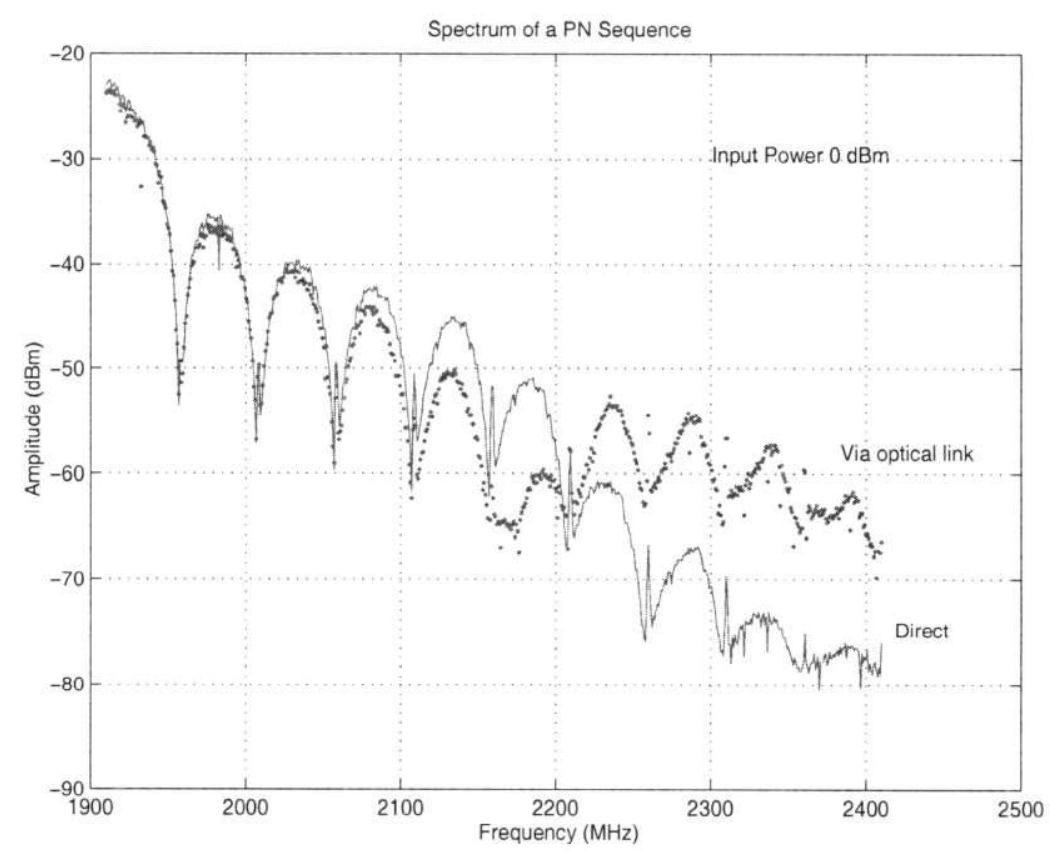

Figure 3.14: Power spectral density of a spread spectrum signal after transmission via the $\mathrm{ROF}$ link at $0 \mathrm{dBm}$ 


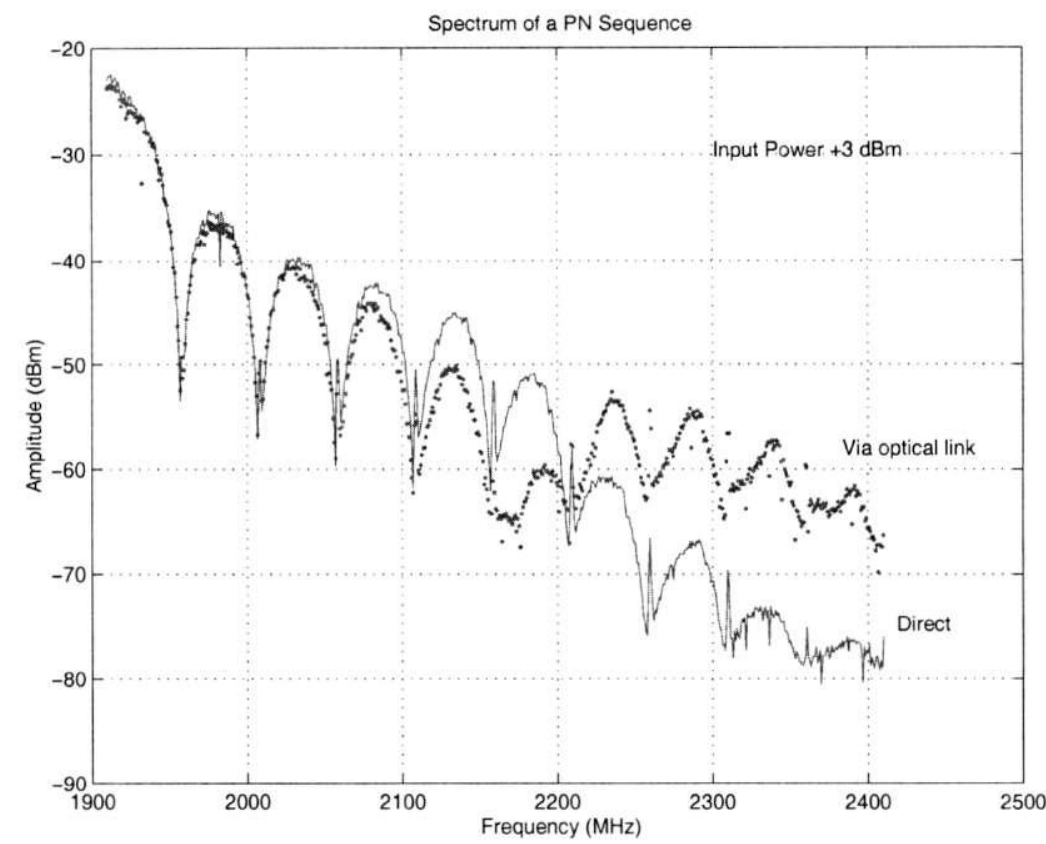

Figure 3.15: Power spectral density of a spread spectrum signal after transmission via the $\mathrm{ROF}$ link at $+3 \mathrm{dBm}$

As shown in Fig. 3.12, at the RF power of $-10 \mathrm{dBm}$, both the input spectrum as well as the output spectrum are almost same up to the sixth null. But after that, the spectrum via optical link shows slightly higher level than the spectrum of the direct signal. Probably this is due to added noise.

As shown in Fig. 3.13, at $-3 \mathrm{dBm}$, both the input spectrum as well as the output spectrum are almost same till the 5th null. However, the spectrum via the optical link has clearly higher energy in the higher order side-lobes than the spectrum of the direct signal.

As shown in Fig. 3.14, at $0 \mathrm{dBm}$, the above phenomenon is more clear. Furthermore, there is a strange phenomenon at fourth and fifth side-lobes. It looks like the ROF link is attenuating the signal around $2200 \mathrm{MHz}$ for some reason at this particular power level.

As shown in Fig. 3.15, at $+3 \mathrm{dBm}$, energy in the first four side-lobes is less for the spectrum of the output signal than the spectrum of the input signal. However, at higher (seventh, eighth etc.) side-lobes the distorted spectrum has almost $15 \mathrm{~dB}$ more energy than the direct spectrum. 
From these measurements we can see that, in general, as the RF power approaches the saturation limit, lower order side-lobes get attenuated while the higher order side-lobes get a boost. Explaining this phenomenon is not easy. A spread spectrum signal has only a single modulated carrier. Therefore, the nonlinearity should generate harmonics and in band AM-AM and AM-PM type distortions only. However, due to the wideband nature of the spread spectrum signal there are more things going on. Since the power density spectrum continues in the frequency axis, we can think of an infinite number of closely stacked frequency components that make up this spectrum. These frequency components instantaneously vary, but their stochastic average gives us this nice looking power spectral density. In this case, in addition to above, there will be significant inter-modulation distortion between all these instantaneous frequency components. Probably, this is the reason for the phenomena we observed from Fig. 3.12 to Fig. 3.15.

\subsection{Deductions from Measurements, Simulations and Analysis}

1. When the RF signal is a few $\mathrm{GHz}$ and the fiber distance is only a few kilometers, there is no time domain distortion due to the fiber dispersion, chirp optical to electrical and electrical to optical conversion processes provided, the $\mathrm{RF}$ power is within the linear range of the ROF link. This is especially true with our measurement set up where, the optical wavelength is $1310 \mathrm{~nm}$, and a single mode DFB laser transmitter is used.

2. The RF frequency decides the effect of time domain dispersion. The bit rate or the chip rate (in a spread spectrum system) of the modulating signal is immaterial, since usually this is only a fraction of the carrier frequency.

3. The ROF link has adequate bandwidth to support several RF carriers when the total power is within the linear range.

4. The dynamic range of the optical link is the most severe constraint. This severely limits the cell size and the number of users because of the nonlinear 
distortions. This, combined with sudden power changes can severely degrade the link performance.

5. The received power level at the detector is too low, about $-30 \mathrm{dBm}$. This needs to be significantly amplified before driving an antenna (30-40 dB) in the downlink. Thus, the nonlinearity (and noise) of the RF amplifiers at the optical receiver introduces further degradation. This also needs to be considered.

6. The $-30 \mathrm{dBm}$ received power is only for one channel. If several RF channels are multiplexed, then this power has to be divided among all of those channels. This will need much higher amplifier gain. For instance, if there are $\mathrm{N}$ channels, then the amplifier gain has to be $10 \log (N)$ dB higher.

7. The AM-PM distortion of the ROF link starts at quite low power level. This should be compensated since all wireless schemes use some form of phase modulation.

8. The noise measurements show a nonlinear phenomena. The noise suddenly increases at the saturation level and shows a frequency dependent phenomenon just before that. This needs to be analyzed further.

9. The measured optical link nominally has a linear range from the noise floor to $0 \mathrm{dBm}$. However, the noise floor depends on the bandwidth of measurement and increases with the bandwidth. This further reduces the available linear range when the modulating signal is wideband. However, the upper limit seems to be constant. 


\section{Chapter 4}

\section{Adaptive Modeling and Nonlinearity Compensation}

From previous chapters, we found that the major concern at PCS band is the nonlinearity of the ROF link that limits the available dynamic range. This limitation exposes itself in several ways. When there is a single RF subcarrier, it saturates and there is AM-AM and AM-PM distortion. When there are two or more subcarriers, the inter-modulation products also come into play and, they start to appear at much lower power levels. When a spread spectrum signal is transmitted, the distortion is very complex.

These observations agree with previously published results in [16] and [33] where, the nonlinearity was the biggest issue. There have been several attempts to solve this limitation. Most of these are electronic linearization approaches using circuit models of the laser [19]. These approaches are discussed in detail in Chapter 1. They lack in flexibility and suffer from device dependency. Therefore, we take a totally different approach where, it is not necessary to exactly characterize the nonlinearity through measurements. Rather, we focus on developing self-learning or adaptive compensation techniques that automatically compensate for the imperfection. This approach will work even if the actual distortion is different from the prediction because of the self-correcting nature.

There are several advantages in using an adaptive signal processing approach. Firstly, this makes accurate measurements of each device unnecessary and hence, overcome device dependency. Secondly, it enables tracking the variation in the link 
characteristics either due to manual modification or due to environmental variations and aging. Thirdly, it is flexible to implement and enable us to introduce asymmetry in complexity, which is very cost effective. Finally, it facilitates baseband compensation. Fortunately, in wireless communications, all the information is transmitted as baseband digital symbols.

\subsection{Basics of Nonlinear Modeling}

Linear models are widely used to represent many systems due to their inherent simplicity. Approximation of mildly nonlinear systems by linear systems under certain constraint is widely used because of this reason. However, certain real life systems such as radio over fiber channel exhibits significant nonlinearity, which cannot be neglected. If such systems are accurately modeled, then a significant performance improvement can be expected.

There are several nonlinear models described in the literature, with varying number of parameters and architecture. Different models are best suited for different applications. Therefore, the first task is to find a model that best suits our application. This should also yield a practically feasible solution and enable us to easily compensate the nonlinearity.

\subsubsection{The Volterra Series Model}

A very common system model that has been employed with relatively good success in nonlinear applications is the Volterra system model. This model expresses the relationship between the output as a function of input only, raised to higher orders as necessary. It does not require knowledge of the internal states. The output $d(n)$ of a causal discrete time Volterra system and its input $u(n)$ are related by ,

$d(n)=h_{o}+\sum_{m 1=0}^{N_{m}} h_{1}\left(m_{1}\right) u\left(n-m_{1}\right)+\sum_{m 1=0}^{N_{m}} \sum_{m 2=0}^{N_{m}} h_{2}\left(m_{1}, m_{2}\right) u\left(n-m_{1}\right) u\left(n-m_{2}\right)+\ldots$

where $h_{p}(\ldots)$ is known as the $p^{\text {th }}$ order Volterra kernel. $N_{m}$ is the memory of the system. Although there are an infinite number of higher order terms, usually they 
are truncated to a few significant orders.

The Volterra series model is the most suitable model for the ROF link for wireless access. The explanation is as follows. The ROF link has several input parameters, such as the optical modulation depth, input RF power and mean optical power. However, for a given ROF link, all other parameters are constant for a reasonable duration except the input RF power, which dynamically changes. This is severe in a multiuser wireless system, since the number of users dynamically changes and the RF power of each user individually varies. This affects the output in that the amplitude and phase of the vector-modulated symbols are distorted as a function of the total RF power through the link. Therefore, the perfect model of the ROF link should be based on the RF power (or RF amplitude) as its input. Furthermore, the measured amplitude and phase response of the ROF link (see Fig. 3.4 and Fig 3.9) show mild nonlinearity and are continuous functions of the input RF power. Hence, a Volterra series model is ideal for modeling the ROF link. Furthermore, the Volterra series model enables adaptive optimization of its weights and handles systems with or without memory.

\subsubsection{Memory of the ROF link}

To model a nonlinear system, the number of states or the order of memory in the system has to be first understood. Considering the ROF link, the nonlinearity of an injection laser has both static and dynamic components. The static (memoryless) nonlinearity is observed directly from the optical power versus current curve. A simple polynomial relationship is assumed for this zero state relationship [9]. In addition to this, semiconductor lasers exhibit dynamic nonlinearity that is intrinsic to the nonlinear photon electron interaction mechanism. Several attempts have been made to characterize this dynamic behavior by solving the laser rate equations [12]. Generally, the results show that even for large signal modulation (modulation index $\geq 0.3$ ), the carrier to third order inter-modulation power ratio becomes frequency independent except near the resonance frequency [33]. Our two-tone test results also agree with this. Therefore, under steady state conditions, the laser can be assumed memoryless.

Being an all pass filter at our modulating frequencies, the single mode optical 
fiber is an inherently memoryless device. The photo detector usually operates in the linear range. Although at instantaneous optical signal peaks, the photodiode may saturate; this sustains for a very short time [42]. Receiver amplifiers exhibit nonlinearity and may have memory. We shall initially assume that their memory is shorter than the sampling period so that, effectively, the whole ROF link can be modeled as a memoryless nonlinear system in the discrete time domain. If the memoryless assumption is found inadequate under some other conditions, it is quite easy to incorporate memory in the Volterra series model.

\subsubsection{Discrete Time Domain Issue}

Discrete time domain analysis is desired for DSP approaches. Therefore, an appropriate sampling frequency to preserve the nonlinear distortion and related issues has to be discussed if the analysis is to be done in discrete time domain. Continuous time domain systems can be represented in discrete time domain by two different methods [37 page 81$]$.

Impulse Invariant Transformation Basically, this is equivalent to sampling by an ideal impulse.

Bilinear Transformation This is a nonlinear mapping from the continuous to discrete time domains. This will not be considered further since, for continuous time domain nonlinear systems, this mapping magnifies the nonlinearity.

Traditionally, it is believed that, to represent a nonlinear system of order $N$, the sampling frequency $\left(f_{s}\right)$ should be higher than twice the highest frequency of the output signal. For example, if the input bandwidth is $B_{i n}$, then,

$$
f_{s} \geq 2 N B_{\text {in }}
$$

However, some recent results [43], [44] based on Zhu's "Generalized Sampling Theorem" [45], show that, it is adequate to sample at the input Nyquist sampling frequency $f_{s} \geq 2 B_{i n}$, rather than the output Nyquist sampling frequency $f_{s} \geq$ $2 N B_{\text {in }}$. This is true for identification and compensation when the nonlinearity is a one-to-one mapping and therefore has an inverse. In this work we assume that 
the sampling frequency is adequate to represent all the signals and systems without aliasing.

\subsection{Modeling the ROF Link}

In this section we describe the adaptive baseband modeling approach for the ROF link. First, we justify a baseband modeling of the occurrences that happen in photonic frequencies. Then, we explain why adaptive modeling is necessary. A Volterra series based modeling procedure is described next.

\subsubsection{Photonic Band to Baseband}

When an analog microwave signal is transmitted through the ROF link, the optical channel impairments contribute to either amplitude or phase (delay) distortion of the microwave signal plus noise. If this microwave signal is vector modulated by digital information then, this distortion will be reflected on the phase and amplitude of these digital symbols. Since the information is transmitted by the phase and amplitude of vector modulated symbols, the ROF link can be adequately modeled at baseband by analyzing the amplitude and phase relationship between input and output symbols. This is also proven by Saleh. In [46], Saleh states that 'a frequency independent memoryless bandpass (inband) amplitude and phase nonlinearity can be completely described by its baseband equivalent'. Being a baseband scheme, the basic filter architecture is independent of the microwave frequency although, the filter coefficients reflect the ROF link at that particular frequency.

\subsubsection{Adaptive Versus Fixed Modeling}

Whenever there is a requirement to process signals that result from operation in an environment of unknown statistics, the use of adaptive filters offer a significant improvement over fixed filter solutions [47]. Adaptive filters are widely used to model many complicated systems. Adaptive modeling is appropriate for ROF links due to the large number of parameters and the variations in their characteristics.

The basic block diagram of an adaptive modeling scheme is shown in Fig. 4.1. 


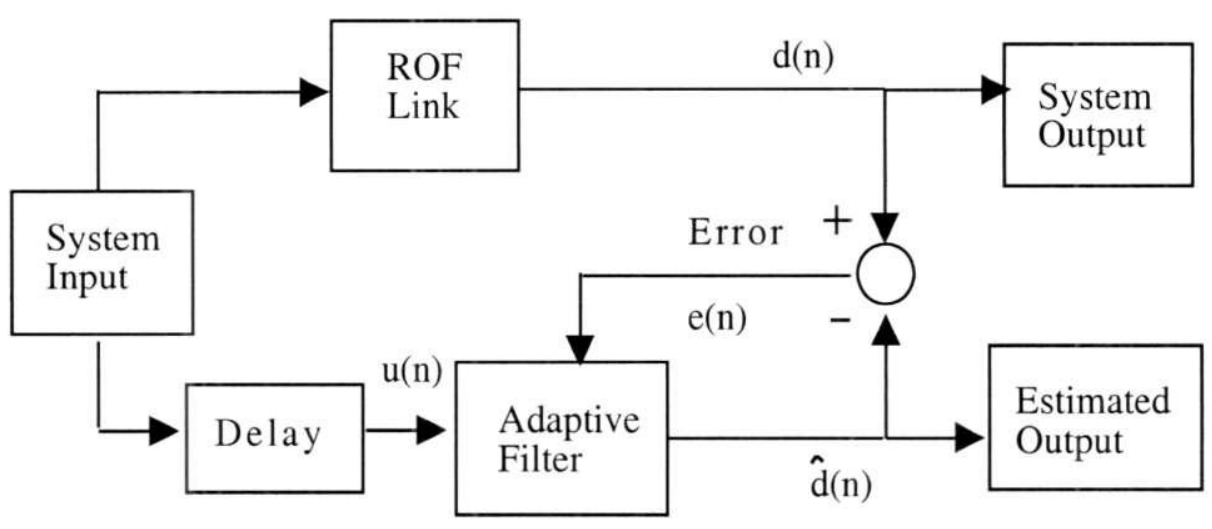

Figure 4.1: Adaptive modeling arrangement of the ROF link

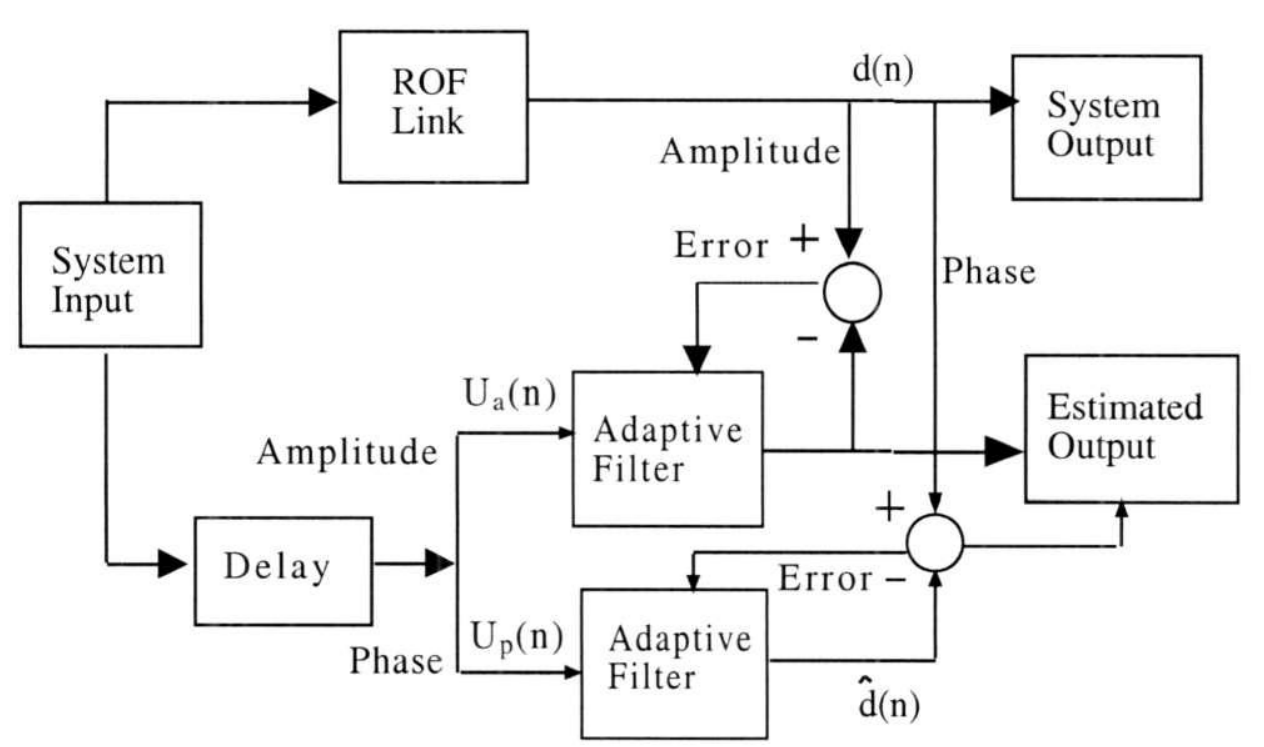

Figure 4.2: Detailed arrangement of the adaptive modeling scheme

The same vector modulated symbols are applied to both the ROF link and the adaptive filter. The respective outputs $d(n)$ and $\hat{d}(n)$ are compared and an error $e(n)$ is generated. This error drives an adaptive algorithm that computes the adaptive filter parameters. A delay is inserted at the input of the filter to match the delay of the ROF link. This delay mainly depends on the fiber length and has to be accurately estimated for better results.

The input symbols are complex, so the filter is complex as well. However, the NLD depends on the input power only and is independent of the input phase. 
Furthermore, amplitude and phase nonlinearity curves have completely different shapes (see Fig. 3.4 and Fig. 3.9). Because of this independence, we can use two separate filters to model amplitude and phase nonlinearities rather than one single complex filter. This arrangement showed better performance in terms of convergence speed and final misadjustment. Such an arrangement is shown in Fig. 4.2 where, $\boldsymbol{U}_{a}(n)$ is the input to the amplitude modeling filter and $\boldsymbol{U}_{p}(n)$ is the input to the phase modeling filter.

\subsubsection{Volterra Kernel Optimization}

When a Volterra system is modeled with a Volterra series adaptive filter, the tap weight vector $\boldsymbol{W}(n)$ of the filter represents the Volterra kernels. The expression for the Volterra series based filter is written as in (4.3), where $w_{k}(\ldots)$ are the filter coefficients and $\hat{d}(n)$ is the estimated output.

$\hat{d}(n)=w_{o}+\sum_{m 1=0}^{N_{m}} w_{1}\left(m_{1}\right) u\left(n-m_{1}\right)+\sum_{m 1=0}^{N_{m}} \sum_{m 2=0}^{N_{m}} w_{2}\left(m_{1}, m_{2}\right) u\left(n-m_{1}\right) u\left(n-m_{2}\right)+\ldots$

Although the weights are multidimensional, they can be stacked as a one-dimensional vector for efficient computation of adaptive algorithms. The length of this vector depends on the order and memory of the model.

$$
\boldsymbol{W}(n)=\left[w_{o}(n) ; w_{1}(n) ; \ldots\right]
$$

The objective of the adaptive filter is to update the coefficient vector $\boldsymbol{W}(n)$ on a sample by sample basis so that the estimation error $e(n)$ between the desired output $d(n)$ and the estimated output $\hat{d}(n)$ is minimized.

$$
e(n)=d(n)-\hat{d}(n)
$$

Any adaptive algorithm can be used for coefficient update. Here the recursive least square algorithm is used due to its better performance over other algorithms with colored (not completely random) inputs. In our application, the adjacent input samples are correlated or colored since the rate of change of the input amplitude is 


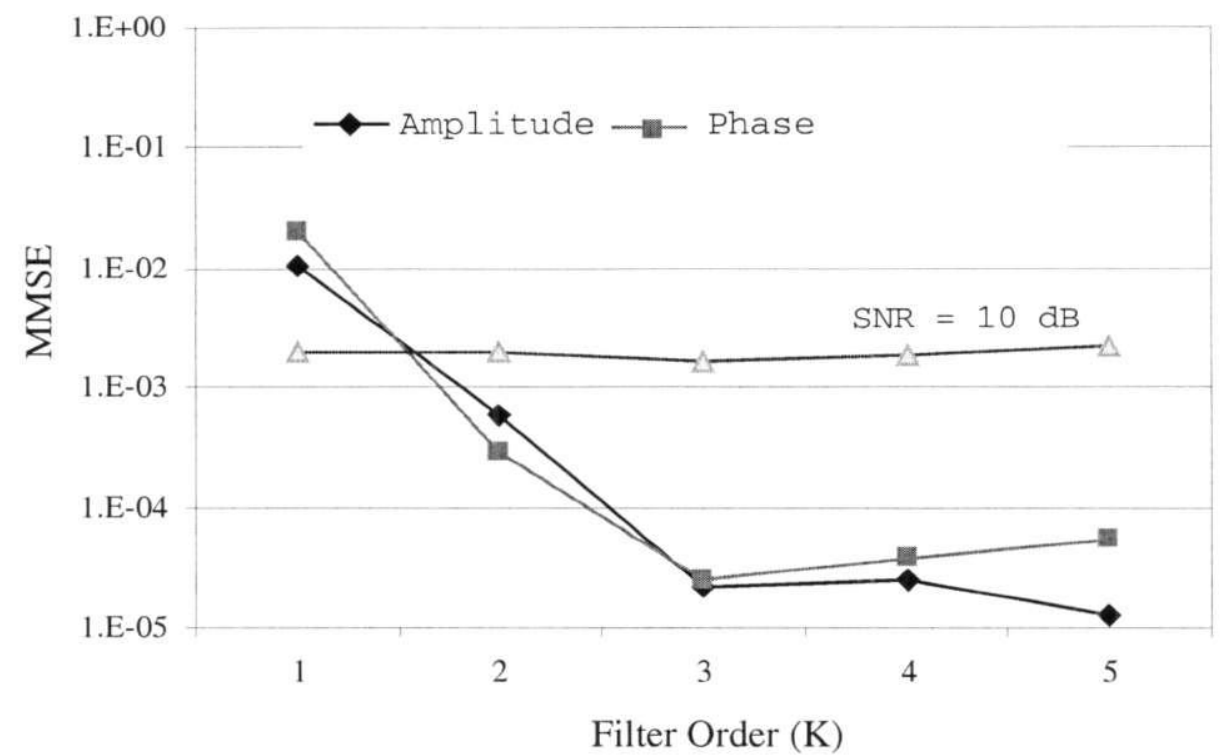

Figure 4.3: Variation of minimum mean squared error (MMSE) with filter order usually much slower than the sampling rate.

To obtain the coefficient vector, the exponentially weighted cost function $J(n)$ in (4.6) is minimized, where $\lambda_{f f}$ is the forgetting factor $\left(0.95<\lambda_{f f}<1\right)$ that controls the rate of convergence.

$$
J(n)=\sum_{k=1}^{n} \lambda_{f f}^{n-k}(d(k)-\hat{d}(k))^{2}
$$

The optimal solution of $\boldsymbol{W}(n)$, which minimizes $J(n)$, is given by [47],

$$
\boldsymbol{W}^{o p}(n)=\Re_{u u}^{-1}(n) \Re_{u d}(n)
$$

where, $\boldsymbol{\Re}_{u u}(n)$ is the auto-correlation matrix of the input vector $\boldsymbol{u}(n)$ and $\Re_{u d}(n)$ is the cross correlation vector between the input vector $\boldsymbol{u}(n)$ and the desired response $d(n)$. The RLS algorithm implements (4.7) recursively. 


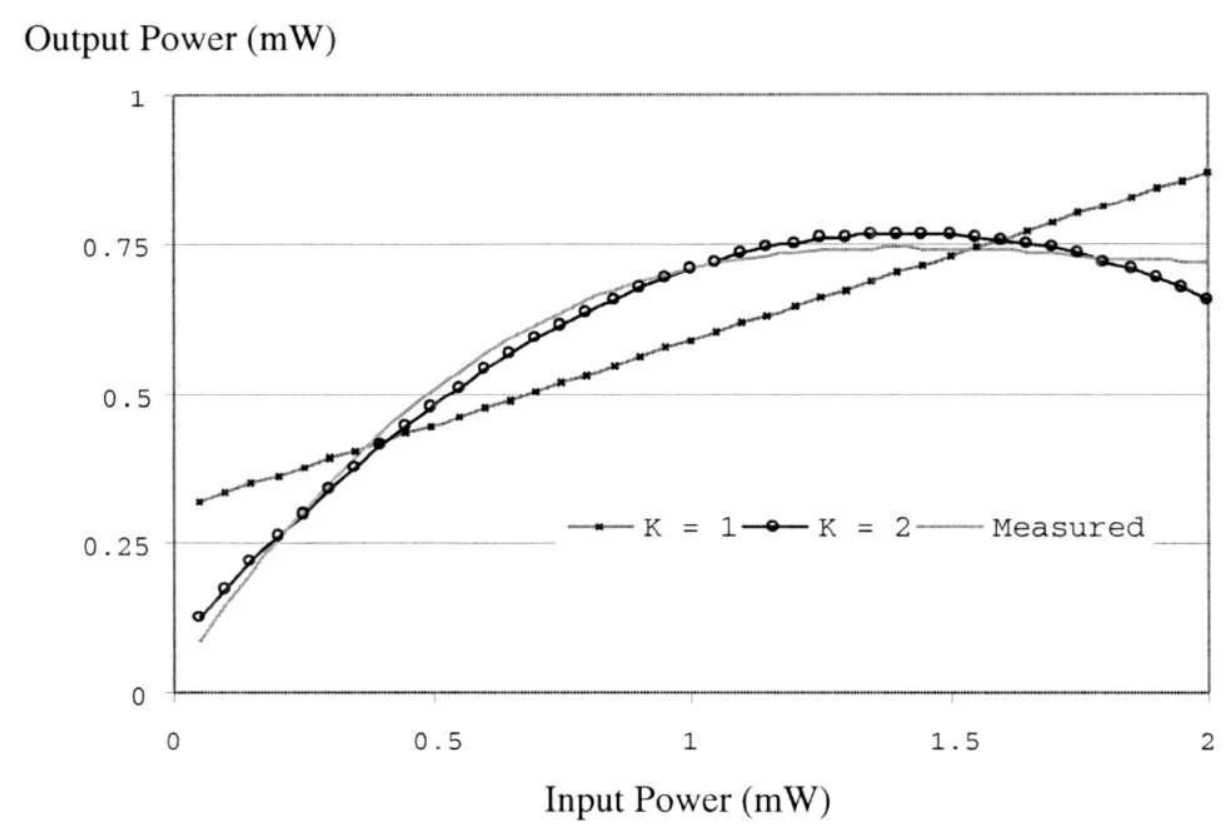

Figure 4.4: Tracking the measured data with different filter orders

\subsubsection{Filter Order and Memory}

The accuracy of the modeling depends mainly on the order and memory of the filter. For linear filters, the order and the memory are the same as the number of filter tap weights. In contrast, for nonlinear filters they are different quantities. The order of the filter indicates the highest order term. $N_{m}$ describes the memory in (4.3), which shows how many adjacent symbols are buffered. Ideally, the order and memory of the filter are same as those of the original system. However, in practice they may be decided based on a trade off between the complexity and accuracy.

The memory of any nonlinear system has to be considered for a complete solution. A pure memoryless system introduces only AM-AM distortion. Strictly speaking, if there is AM-PM distortion that means there is memory. However, if the memory is small enough so that the time constants of the system is much smaller than the period of the modulation frequency, then the system is considered as a quasi-memoryless system [48]. In a quasi-memoryless system, at a certain instant the amplitude and phase of the output are functions of the input power at that instant only. For systems with larger time constant, the modeling should 
consider the effect of previous sampling instances also. Strictly speaking, ROF link has memory. However, for up to several Mbps rate it is modeled as a memoryless channel [9]. This issue was discussed in detail in Section 4.1.1.1. As a result, we have considered a memoryless higher order filter modeling in this section.

The Minimum Mean Squared Error (MMSE), which is the minimum value of the mean square of error $E\left[e^{2}(n)\right]$, is a good indication of modeling accuracy. When the mean squared error has attained this steady state value, the filter is considered to have converged. The MMSE is used here as a criterion to select the filter order.

\subsubsection{Simulation and Results}

The experimental AM-AM and AM-PM data is used to run the simulation and to train the filters. Matlab ${ }^{T M}$ based Simulink ${ }^{T M}$ software was used for the adaptive filter realization. The signal to noise ratio (SNR) is set to $20 \mathrm{~dB}$ during simulation.

\subsubsection{Modeling the Amplitude Response}

Referring to Fig. 4.2, the amplitude gain of the link depends on the amplitude of the input signal only. Therefore, the input of the amplitude modeling filter $\boldsymbol{U}_{a}(n)$ is taken as the vector in (4.8), where $K$ is the order of the filter and $a(n)$ is the amplitude of the transmitted symbols. $p(n)=a^{2}(n) / 2$, is the power assuming a one ohm resistor.

$$
\boldsymbol{U}_{a}(n)=\left[1 ; p(n) ; p^{2}(n) ; \ldots \ldots . p^{K}(n)\right]
$$

The minimum mean squared error (MMSE) is computed with different filter orders. The MMSE versus filter order is shown in Fig. 4.3 where, $K=3$ gives a MMSE of $2.1291 \times 10^{-5}$. Increasing $K$ further, only marginally decreases the MMSE. Hence, a filter of order three is adequate to model the AM-AM modulation. Fig. 4.4 shows the measured and estimated filter output responses for $K=1$ and 2. When $K \geq 3$, the estimated output coincides with the measurement. From Fig. 4.4 , it is seen that even at $K=2$, the filter output tracks the measurements very closely.

The tap weight vector after convergence is given by (4.9). The elements of the vector are the coefficients of a third order polynomial. The constant shift is 
approximately 0.02 , which is the output when the input is zero. Note that this is equal to the noise floor.

$$
\boldsymbol{W}=[0.01966 ; 1.3275 ;-0.7914 ; 0.1509]
$$

\subsubsection{Modeling the Phase Response}

Referring to Fig. 4.2, the output phase of the link depends, linearly on the input phase and, non-linearly on the input amplitude. The input to the phase modeling filter $\boldsymbol{U}_{p}(n)$ is taken as the following vector.

$$
\boldsymbol{U}_{p}(n)=\left[1 ; p(n) ; p^{2}(n) ; \ldots \ldots . p^{K}(n), \theta(n)\right]
$$

The minimum mean squared error (MMSE) is computed with different filter orders to determine the appropriate filter order. This is also shown in Fig. 4.3. From the figure, again it is seen that $K=3$ gives a MMSE of $2.3949 \times 10^{-5}$. Increasing $K$ further, does not decrease the MMSE. Therefore, a third order filter is adequate to model the AM-PM distortion too. The tap weight vector is given by (4.11). Note that the weight applied to the input phase is unity (last element of $\boldsymbol{W}$ ) indicating that the output phase is a linear function of the input phase. The constant term is 2.5159 radian or $144^{\circ}$. This is the phase shift when the input power is almost zero, Fig. 3.4.

$$
\boldsymbol{W}=[2.5159 ;-1.5765 ; 0.6060 ;-0.0276 ; 1.0001]
$$

\subsubsection{Effect of Noise}

The modeling accuracy depends on the signal to noise ratio as well. It is observed that even at low SNR, the filter tracks the mean value of the signal trace. However, the MMSE is high because of high noise power. From Fig. 4.3, again it is seen that when the SNR is low ( $\mathrm{SNR}=10 \mathrm{~dB}$ ), the MMSE is constantly high irrespective to the order of the filter. 


\subsection{The Compensation}

Once the nonlinearity has been modeled, a suitable compensation scheme can be devised. Now, there are two links involved. For the uplink, the compensation is done at the central base station because that is the receiving end. For the downlink also, the compensation should be done at the central base station. This is because; out-of-band emissions will violate FCC regulations and portable units require a much complex equalization scheme if the radio transmission occurs with nonlinear distortion. This arrangement gives asymmetry in complexity. That means the signal processing complexity for the up and down links is shifted to the central base station. It is desirable to do more processing at the central base station because; the cost of the central base station is shared among many users. Such an asymmetric arrangement is shown in Fig. 4.5.

The system block diagram shown in Fig. 4.5 is for a single micro/pico cell and a single portable. In the uplink, the RAP receives wireless signals from the portable unit. After amplification, the RF signal intensity modulates the laser $(\mathrm{E} / \mathrm{O})$, which generates the sub-carrier modulated optical signal. At the other end of the fiber, which is in the central base station, the optical receiver detects the radio signal $(\mathrm{O} / \mathrm{E})$. This signal is then bandpass filtered, amplified and demodulated to baseband where the post-compensation filter corrects NLD.

In the downlink, complex baseband symbols emanating from the central base station are appropriately predistorted by the pre-compensation filters before RF modulation. The RF signal then intensity modulates the laser for transmission through the fiber. At the other end of the fiber, which is in the RAP, the RF signal is extracted from the optical carrier. Then it is suitably amplified and then radiated into the wireless channel.

Note that the RAP acts as a wireless receiver as well as an optical transmitter in the uplink. In the downlink, it acts the other way round. In addition, more amplification is required in the downlink due to wireless transmission requirements. A suitable frequency or time duplex scheme is assumed. 


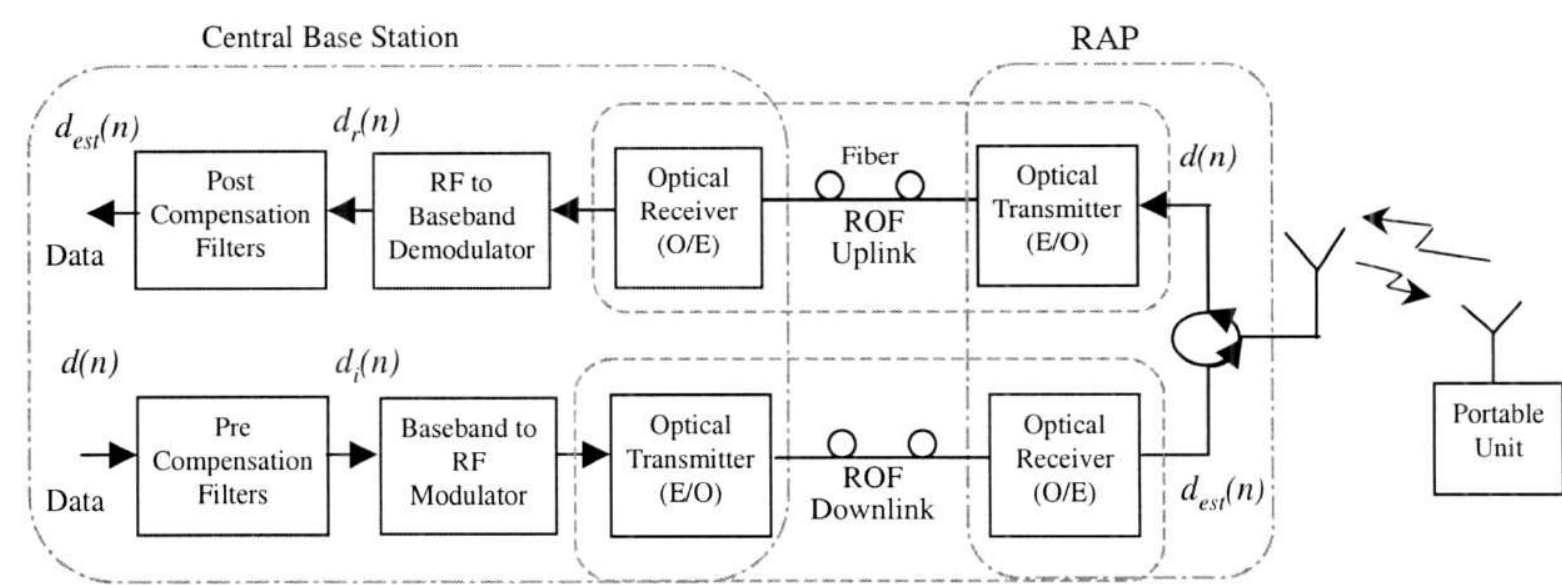

Figure 4.5: Asymmetric compensation arrangement of the fiber-wireless access scheme

\subsubsection{Predistortion Versus Post-Compensation}

For nonlinearity compensation using practical digital filters, the inverse of $F($. should be realizable where, $F($.$) is the original nonlinear function. This imposes$ different constraints depending on whether the compensation is done before or after the nonlinear channel.

\subsubsection{The Predistortion}

For linearization by predistortion, the constraint in (4.12) has to be met where, $G($.) is the inverse nonlinear function. Generally, linearization by predistortion is more difficult to achieve than by post-compensation, because in this case the nonlinearity comes after the compensation filter.

$$
F(G(x)) \propto x
$$

In theory, both amplitude and phase distortions can be corrected by predistortion. For saturating amplitude nonlinearities, however, correction by predistortion is more difficult. For example, we note from Fig. 3.4 that, the amplitude response is almost linear up to $0.7 \mathrm{~mW}$ and then saturates. Entirely correcting the saturation is difficult with predistortion. To overcome saturation the predistortion filter suggests very high input amplitude that would kill the laser. Therefore, it should be done 
with some amplitude back off.

On the other hand, considering the phase response, even if it is quite nonlinear from the origin (Fig. 3.4), it can be corrected without much difficulty using a predistortion scheme. This is because of two reasons. First, any amount of phase correction can be added to the input phase without worrying about damaging the laser. Secondly, the phase distortion is a function of input RF power and, the input RF power is exactly known at the predistortion stage.

\subsubsection{The Post-Compensation}

In post-compensation, the appropriate correction is added to the received signal depending on the received power. This is similar to predistortion, except that the correction is done after the ROF link. For example if $G($.$) is the inverse of F($.$) ,$ then for linearization by post-compensation, the constraint in (4.13) has to be met.

$$
G(F(x)) \propto x
$$

This condition can be met for saturating type $F($.$) , by adding more gain in the$ saturating region (and by reducing the gain at the lower end if necessary) without damaging the laser. In practice, saturating type nonlinearity is more common. Nevertheless, in case of a different type of nonlinearity, like an exponentially increasing $F($.), it is again possible to attenuate more in the high gain region to attain linearity, provided that the large amplitude can be safely handled. This type of exponentially increasing nonlinearity, although not common in communication system, may arise from avalanche type devices. One example is the avalanche photodiode [40].

When post-compensation is to be done, there are some additional issues. The input to the Volterra filter is the received power in post-compensation. However, the distortion is a function of the transmitted power and the received power is different from the transmitted power (because of the nonlinear distortion). Using this distorted received power, as the reference does not yield a very good performance. This is especially true in the saturated region where the correlation between the input and output powers is low. 


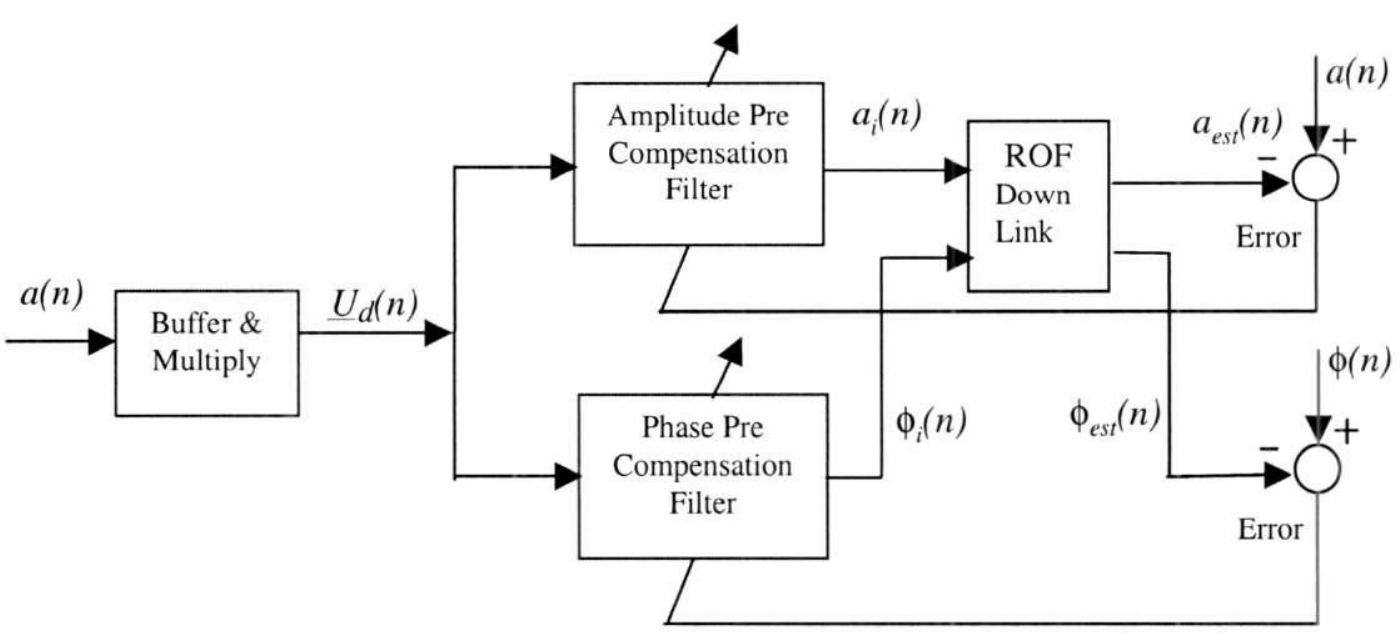

Figure 4.6: Training arrangement for the predistortion filters

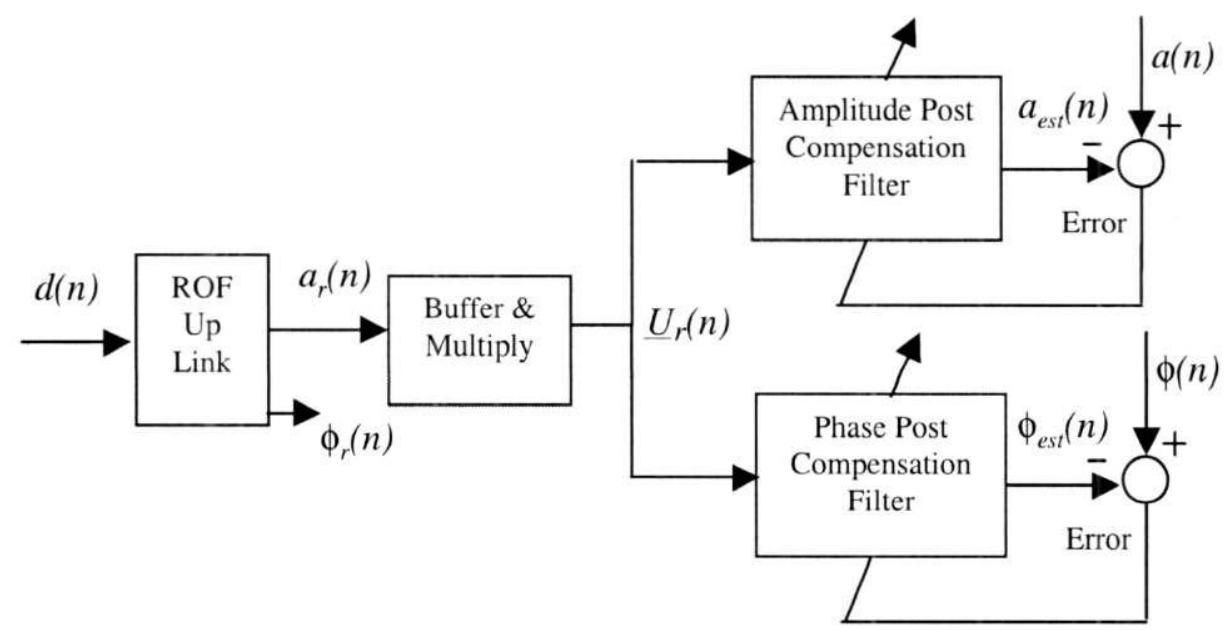

Figure 4.7: Training arrangement for the post-compensation filters

\subsubsection{A Unified Analysis of Asymmetric Compensation}

In this section, a unified analysis is given for the optimization of both post-compensation and predistortion filters. For nonlinearity compensation, the ROF link is inversemodeled with an adaptive filter that corresponds to another Volterra series. After convergence, the tap weights of this filter formulate a higher order function, which is an inverse of the original function. A description on weight optimization using the RLS adaptive algorithm is given in Section 4.2. The objective of the adaptive algorithm is to update the weight vector $\boldsymbol{W}(n)$ on a sample by sample basis so that 
the estimation error $e(n)$ between the desired output $d(n)$ and the estimated output $d_{e s t}(n)$ is minimized in a mean-squared sense.

$$
e(n)=d(n)-d_{e s t}(n)
$$

An adaptive algorithm is applied to minimize the mean square of this error.

This scheme is applied to the system in Fig. 4.5. The pre-compensation arrangement is shown in Fig. 4.6 and the post-compensation arrangement is shown Fig. 4.7. The 'buffer \& multiply' blocks create the input vector to the adaptive filters. We assume that, except for the ROF link, all other elements from the portable to the base station are ideal. Under this assumption, the distortion of the received sequence is only due to the ROF link. Now, consider a training sequence $d(n)$ with known amplitude $a(n)$ and phase $\phi(n)$, that is,

$$
d(n)=a(n) e^{j \phi(n)}
$$

In the uplink, $d(n)$ is transmitted from the $\mathrm{RAP}^{1}$. After transmission through the ROF link, the received symbol $d_{r}(n)$ has a distorted amplitude $a_{r}(n)$ and a phase error $\theta_{r}(n)$ due to the ROF link

$$
d_{r}(n)=a_{r}(n) e^{j\left[\phi(n)+\theta_{r}(n)\right]}
$$

In the downlink, $d(n)$ is transmitted from the central base station. After predistortion the intermediate symbol $d_{i}(n)$ has an amplitude $a_{i}(n)$ and a phase shift $\theta_{i}(n)$ at the input of the ROF link, that is,

$$
d_{i}(n)=a_{i}(n) e^{j\left[\phi(n)+\theta_{i}(n)\right]}
$$

In both links, the output of the combined system (filter plus link) is $d_{\text {est }}(n)$.

${ }^{1}$ If it is transmitted from the portable, then we should assume that the $d(n)$ is available at the RAP without any distortion in the wireless channel. That means, the wireless channel is an AWGN channel. 
This has an amplitude $a_{e s t}(n)$ and a phase shift $\phi_{e s t}(n)$.

$$
d_{e s t}(n)=a_{e s t}(n) e^{j\left[\phi(n)+\theta_{e s t}(n)\right]}
$$

This $d_{\text {est }}(n)$ is compared with $d(n)$. In the uplink, the $d_{\text {est }}(n)$ is available at the central base station and it is compared with a stored copy of $d(n)$. However, in the downlink, the $d_{\text {est }}(n)$ is available only at the RAP and has to be transmitted back to the base station. This has some practical difficulties that are discussed in the Section 4.4. In any case, since the filters try to cancel the distortion of the link, after convergence $d_{e s t}(n)$ closely resembles $d(n)$ and the error $e(n)$ in (4.14) approaches zero. That means $\theta_{\text {est }}(n)$ approaches zero and $a_{\text {est }}(n)$ approaches $a(n)$. If we define $\phi_{\text {est }}(n)=\phi(n)+\theta_{\text {est }}(n)$ then, $\phi_{\text {est }}(n)$ approaches $\phi(n)$. The RF powers corresponding to these symbols, assuming one-ohm resistance at each location, are given by following equations.

$$
\begin{gathered}
p(n)=a^{2}(n) / 2 \\
p_{r}(n)=a_{r}^{2}(n) / 2
\end{gathered}
$$

Now, the adaptive filters are designed to apply compensation to the baseband symbols as a function of their RF power. The use of two separate filters for amplitude and phase compensation yields better results because; the amplitude and phase nonlinearities are quite independent of each other (Fig. 3.4). The two filters have exactly the same architecture except that, for amplitude compensation, the system output is compared with $a(n)$ while for phase compensation it is compared with $\phi(n)$. This is true in both up and down links. These arrangements are shown both in Fig. 4.6 and Fig. 4.7. For the data rate considered, we decided to use a memoryless filter. In that case, $N_{M}$ is set equal to zero and, the Volterra series expression in 4.3) simplifies to a polynomial equation. Now, define the tap input vector $\boldsymbol{U}_{r}(n)$ to the post-compensation filter as,

$$
\boldsymbol{U}_{r}(n)=\left[1 ; p_{r}(n) ; p_{r}^{2}(n) ; \ldots ; p_{r}^{N_{r}}\right]^{T}
$$

$\boldsymbol{U}_{r}(n)$ is the same for both amplitude and phase filters. However, their orders $N_{r}$ are not necessarily the same. This input vector is multiplied by the weight vector 
$\boldsymbol{W}_{a}(n)$ of the amplitude post-compensation filter to obtain the output $a_{\text {est }}(n)$ as in (4.22). Similarly, $\boldsymbol{U}_{r}(n)$ is multiplied by the weight vector $\boldsymbol{W}_{\phi}(n)$ of the phase post-compensation filter to obtain the output $\phi_{\text {est }}(n)$ as in (4.23).

$$
\begin{aligned}
& a_{e s t}(n)=\boldsymbol{W}_{a}(n) \boldsymbol{U}_{r}^{T}(n) \\
& \phi_{\text {est }}(n)=\boldsymbol{W}_{\phi}(n) \boldsymbol{U}_{r}^{T}(n)
\end{aligned}
$$

That is, the output of the filter is a polynomial function of the input RF power with appropriate weights. Note that in post-compensation, the ROF link output power $p_{r}(n)$ is used for filter input while the distortion is a function of the ROF link input power $p(n)$. This issue is further discussed in Section 4.3.3. The $a_{\text {est }}(n)$ and $\phi_{\text {est }}(n)$ are then compared with a stored copy of $a(n)$ and $\phi(n)$, respectively, to create error terms as shown in Fig. 4.7.

In the downlink, the tap input vector $U_{d}(n)$ to the pre-compensation filter is defined as,

$$
\boldsymbol{U}_{d}(n)=\left[1 ; p(n) ; p^{2}(n) ; \ldots ; p^{N_{d}}\right]^{T}
$$

Note that in contrast to the uplink, the $\boldsymbol{U}_{d}(n)$ consists of the RF power of the original symbols $d(n)$, which makes pre-compensation easier. This input vector is multiplied by the weight vectors of amplitude and phase pre-compensation filters to obtain the outputs $a_{i}(n)$ and $\phi_{i}(n)$ respectively as shown in Fig. 4.6. However, note that now these $a_{i}(n)$ and $\phi_{i}(n)$ are going through the ROF link to obtain $a_{e s t}(n)$ and $\phi_{\text {est }}(n)$ before comparison.

\subsubsection{Simulation and Results}

Measurements were taken on a ROF link that is described in Chapter 3. The optical link loss is compensated with a high-gain receiver amplifier so that the overall gain is approximately one. Vector-modulated symbols were transmitted via the simulated ROF link to train the filters as shown in Fig. 4.6 and Fig. 4.7; 2000 iterations were run each time. The forgetting factor $\lambda_{f f}$ was set to unity assuming a stationary environment. Although the measurements were taken up to $2.5 \mathrm{~mW}$, the simulation was run by changing $p(n)$ from zero to a maximum value $p_{\max }(\leq 2.5 \mathrm{~mW})$ because, 


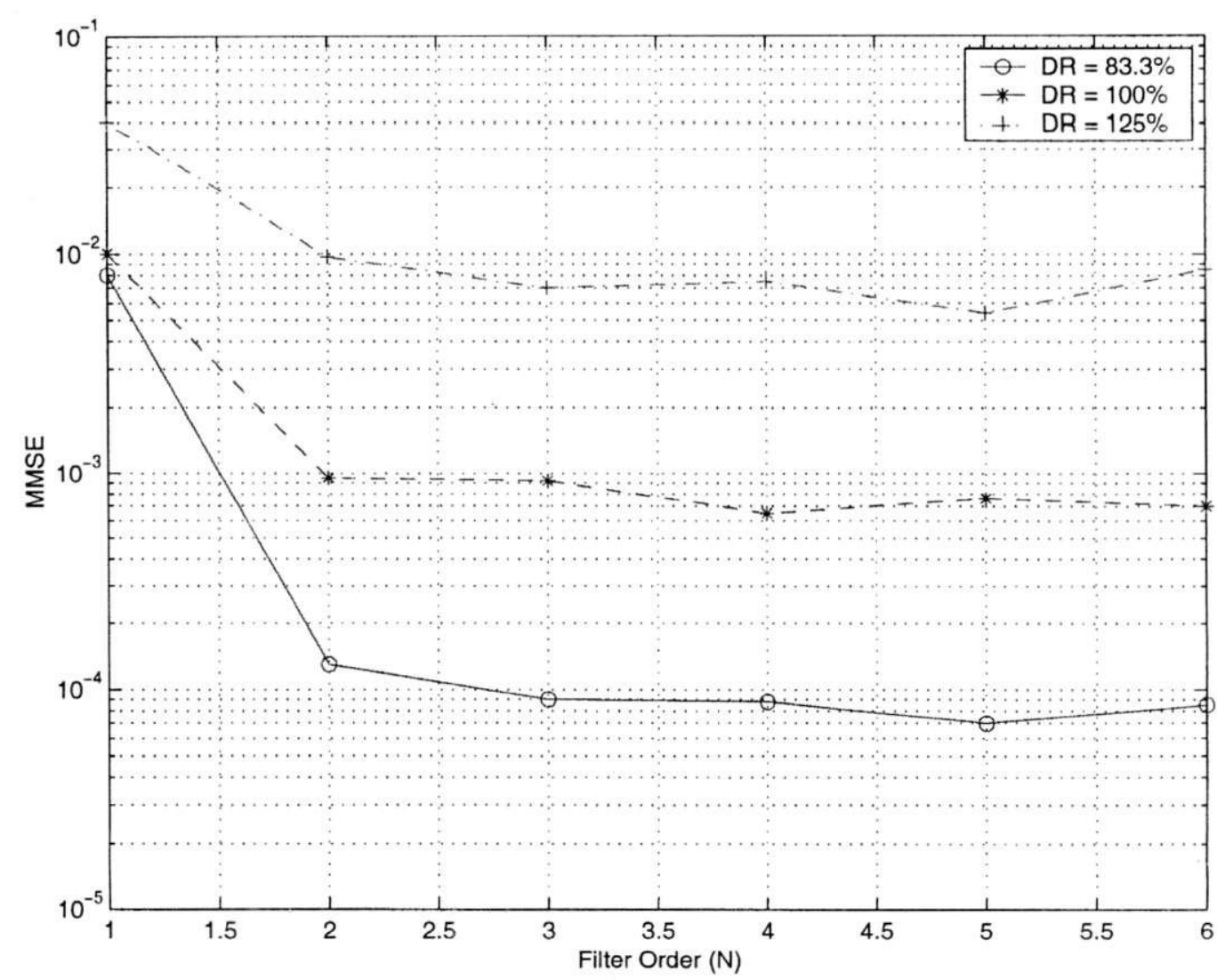

Figure 4.8: Effect of the dynamic range on compensation

the convergence was a function of $p_{\max }$. We refer to this range as the dynamic range (DR). Furthermore, from Fig. 3.4, the output power saturates softly until $0.74 \mathrm{~mW}$. This is achieved when the input power is $1.2 \mathrm{~mW}$. After this point, the output power does not increase with increase in the input. We refer to this $1.2 \mathrm{~mW}$ input as the clipping limit. The actual dynamic range is normalized with respect to this $1.2 \mathrm{~mW}$ to generalize the results.

\subsubsection{Amplitude Pre-Compensation}

For amplitude pre-compensation, the optimum filter order was determined by running the filter with different orders within different dynamic ranges as before. It was found that at least a third order filter is needed for adequate compensation. However, now a notable $30 \%$ back off is required to achieve good linearity. Without back off, the combined output is still distorted. Fig. 4.9 shows uncompensated, 


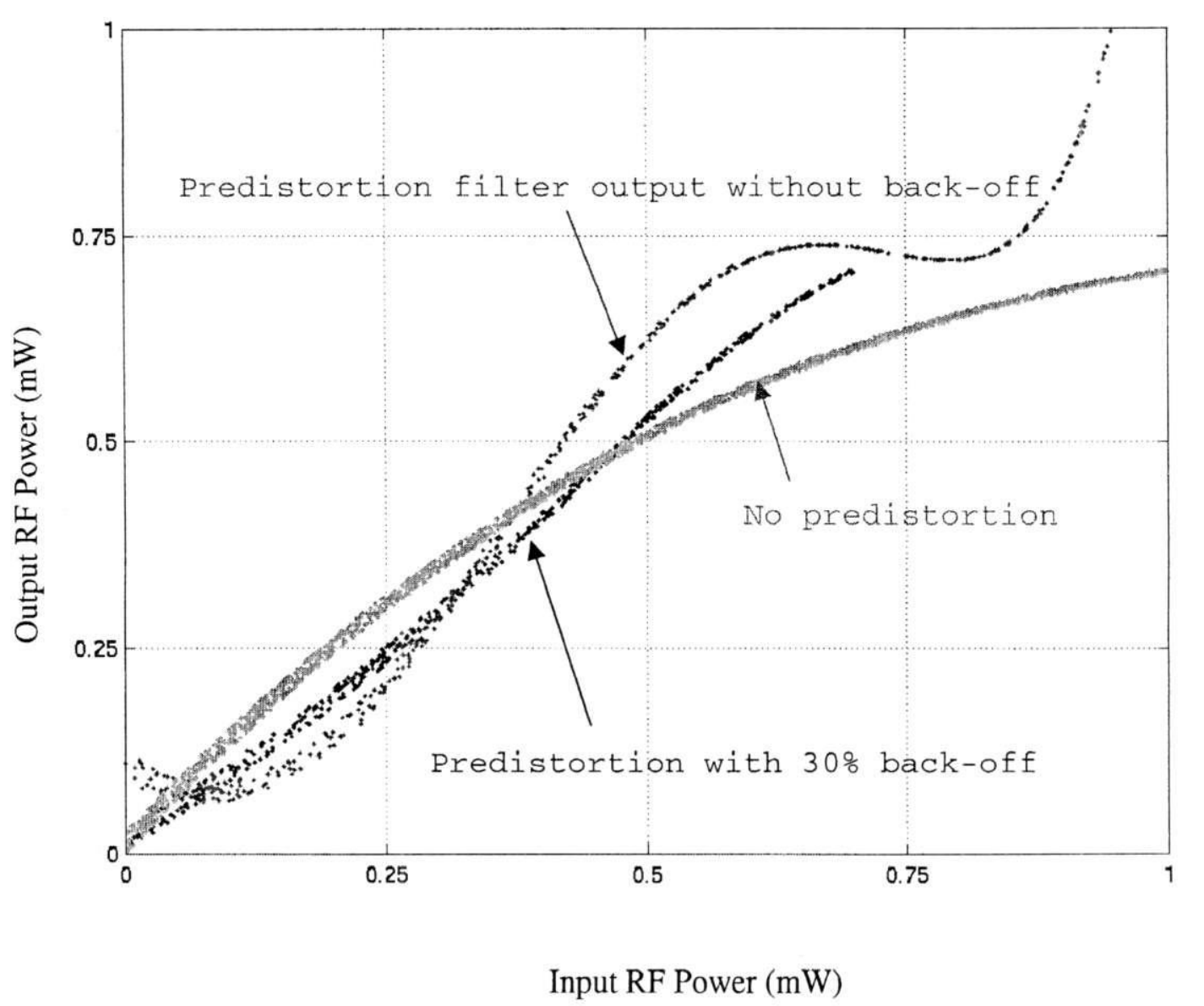

Figure 4.9: Amplitude pre-compensation using a third order filter

linearized and the filter output powers as a function of the system input power. It can be seen that, to overcome saturation, the filter suggests a very high output power, $p_{i}(n)=d_{i}^{2}(n) / 2$, that exponentially increases with the input power $p(n)$. This exceeds the specified maximum power of the laser much before the clipping limit. Thus, a significant back off is required for pre-compensation. However, with the back off, the linearity achieved is good.

\subsubsection{Phase Pre-Compensation}

Phase pre-compensation works well giving an error less than $+/-0.01$ radians with just a second order filter. Fortunately, the phase distortion is a function of input $\mathrm{RF}$ power to the laser and the same RF power is available for the filter for reference. 


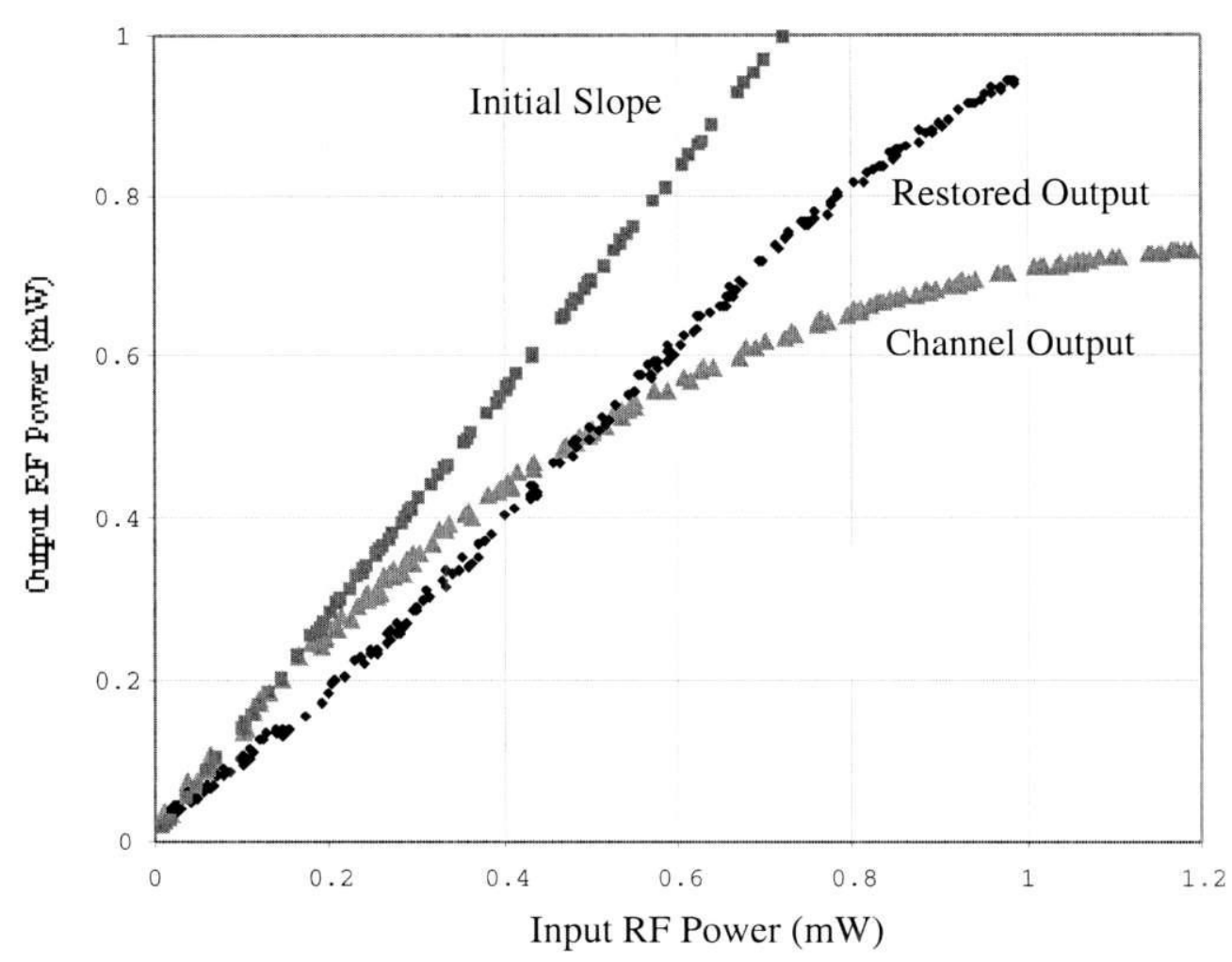

Figure 4.10: Amplitude post-compensation with a second order filter

Furthermore, phase is easily adjusted without concerning about damaging the laser. Therefore, phase pre-compensation is the easiest among all the four tasks.

\subsubsection{Amplitude Post-Compensation}

The convergence of the filters strongly depends on the dynamic range and filter order $N_{r}$. To study the effects, the simulation was run with different $N_{r}$ and DR. The Minimum Mean Squared Error (MMSE), is plotted versus $N=N_{r}$ in Fig. 4.8. The MMSE achieved a steady state within the first 400-500 symbols. Fig. 4.8 shows that the lowest MMSE is achieved when the input dynamic range is within $83.3 \%$ of the clipping limit; this is a $16.7 \%$ back off. In this case, however, a second order filter is sufficient.

The linearized amplitude response with a second order filter as well as the uncompensated channel output are shown in Fig. 4.10. The Channel output has an 


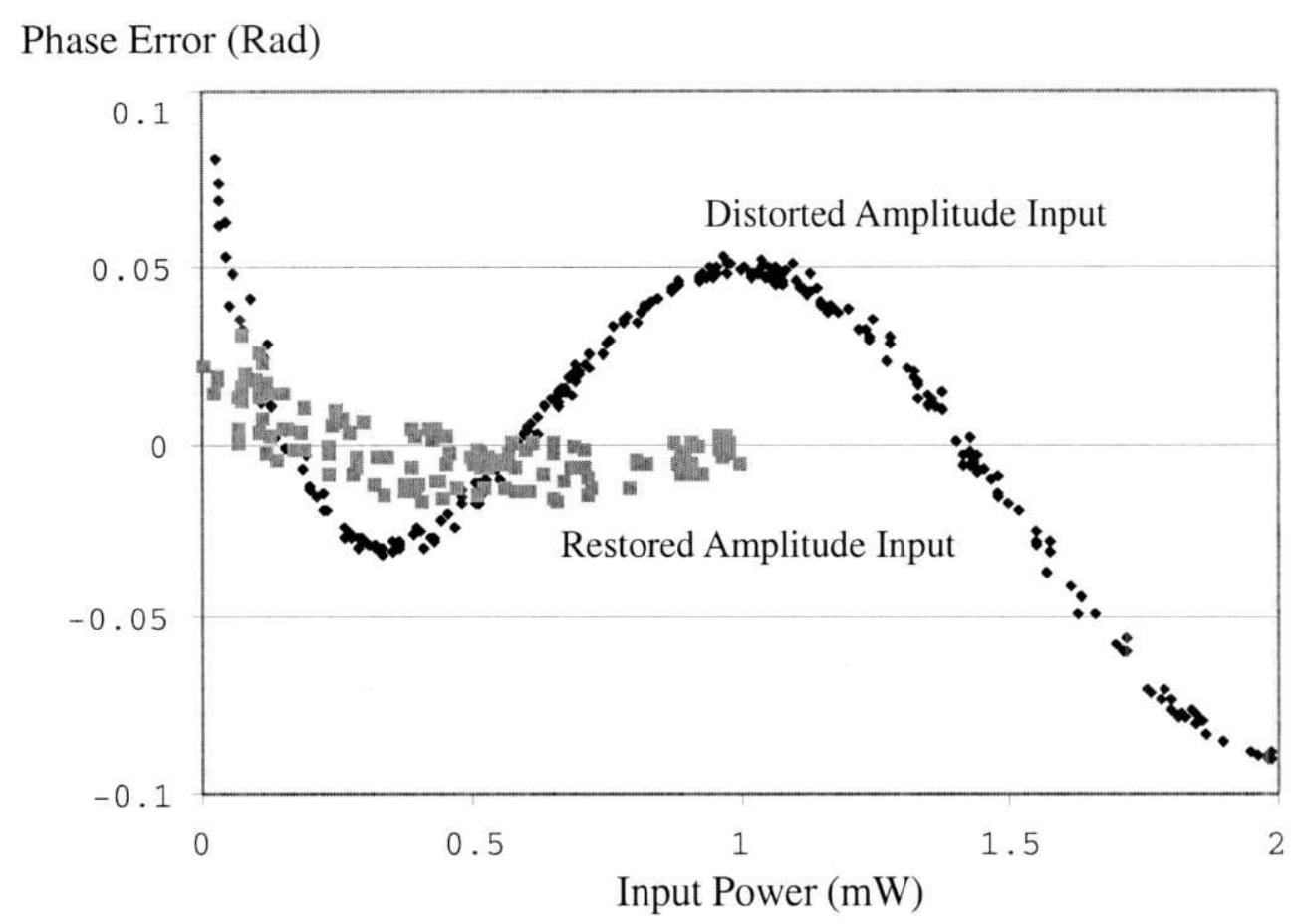

Figure 4.11: Phase post-compensation using a third order filter

initial slope of about 1.38 , which is shown by an asymptotic straight line. However, it starts to saturate rapidly, attaining a unity gain at $0.5 \mathrm{~mW}$ and fully saturating at $1.2 \mathrm{~mW}$ input. On the other hand, the restored output is almost linear up to 0.9 $\mathrm{mW}$ input and shows a small deviation after that.

\subsubsection{Phase Post-Compensation}

It was also found that a third order filter is sufficient for phase post- compensation. The error between the original phase and the restored phase is less than $+/-0.1$ radian up to $2 \mathrm{~mW}$ or $167 \%$ of the clipping limit (Fig. 4.11). This is adequate for most practical applications and can be further reduced if a lower dynamic range is used during training. However, when the input amplitude is high, the error increases. This is because when the input amplitude is high there is less correlation between $p(n)$ and $p_{r}(n)$. Note that, the filter uses the received power $p_{r}(n)$ to 


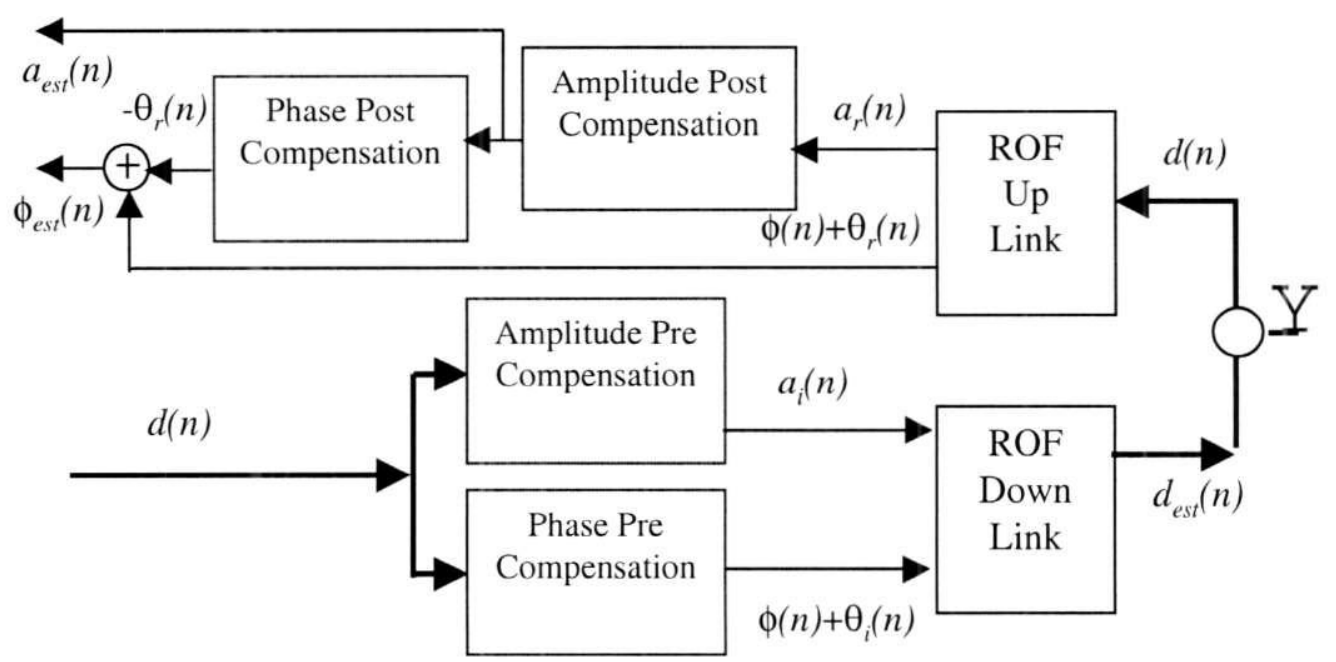

Figure 4.12: The final asymmetric compensation arrangement

\begin{tabular}{|c|c|c|}
\hline Compensation Type & Amplitude & Phase \\
\hline Post-Compensation & $\begin{array}{c}\text { Works well with } \\
\text { a small back off }\end{array}$ & $\begin{array}{c}\text { Works fine when the } \\
\text { restored amplitude is } \\
\text { used for estimation }\end{array}$ \\
\hline Pre-Compensation & $\begin{array}{c}\text { Considerable back off } \\
\text { is required due to } \\
\text { the power handling } \\
\text { capability of the laser }\end{array}$ & $\begin{array}{c}\text { Works well since the } \\
\text { distortion is a function } \\
\text { of the input power and } \\
\text { the filter uses the same } \\
\text { input power for estimation }\end{array}$ \\
\hline
\end{tabular}

Table 4.1: A summary of asymmetric memoryless compensation schemes

estimate the phase distortion $\theta(n)$ while, $\theta(n)$ is a function of transmitted power $p(n)$.

To overcome this issue, we modified the arrangement of post-compensation filters. First, the amplitude compensation filter is deployed to restore the amplitude from the received power $p_{r}(n)$. Then, the restored amplitude is used as the input to the phase compensation filter (Please refer the final arrangement in Fig. 4.12). In this case, the performance is much better, with a phase error less than $+/-0.03$ radian. This error is also shown in Fig. 4.11. However, now the useful dynamic range is reduced to one $\mathrm{mW}(83.3 \%)$. 


\subsection{Concluding Remarks}

In the first part of this chapter, we described a baseband-modeling scheme for the radio over fiber link. Our simulation results show that third order filters are adequate to model both the phase and amplitude nonlinearity. This conclusion agrees with the previous modeling attempts in the sense that, under steady state conditions, both the laser [16] as well as RF power amplifiers [48] have been modeled with third order approximations.

In the second part of this chapter, an asymmetric nonlinearity compensation scheme is described. The results are summarized in Table 4.1. Final arrangement of the compensation filters is shown in Fig. 4.12.

This adaptive modeling and compensation approach is independent of ROF link parameters such as radio frequency, optical modulation depth, optical wavelength, fiber length etc. However, the order of the filters must be adequate for the given dynamic range. Although, the discussion is focused on directly modulated links, it is readily extensible to other types of optical modulation schemes.

In this work, we assumed a memoryless, mild nonlinearity and used simple polynomial type filters. However, when this is not applicable, advanced compensation schemes have to be investigated. Furthermore, we have assumed all the elements other than the ROF link are ideal in order to isolate the problem. Impairment of the other elements, especially the wireless channel has to be considered for a complete solution. Another issue is training the pre-compensation filter. This requires the estimated outputs $\left(a_{\text {est }}(n)\right.$ and $\left.\phi_{\text {est }}(n)\right)$ to be transmitted back to the central base station. This is a challenging task because of the distortion in the ROF uplink. One way of approaching this problem is to first accomplish the uplink compensation and then attempt the downlink compensation. These issues are discussed in Chapter 9. Finally, a constant noise power is used during simulation. Nevertheless, a nonlinear increment in the ROF link noise was observed when the signal power was high. This issue needs further investigation. 


\section{Chapter 5}

\section{Noise and BER Analysis of A DS/CDMA Fiber Wireless System}

In the previous chapter, we investigated various nonlinearity compensation techniques for the ROF link. This is done either in a predistortion configuration or in a post-compensation configuration. In this chapter we investigate the BER performance of a direct sequence code division multiplex access (DS/CDMA) scheme that deploys the ROF link to provide wireless access to multiple users. The focus of this chapter is to investigate the effect of various noise mechanisms of the ROF link, mainly the shot, thermal and relative intensity noise (RIN) mechanisms, on the BER performance, in addition to interference. Note that, of these noise mechanisms, shot noise is a function of the mean optical power while the RIN is a function of the instantaneous optical power. Therefore, the RIN increases with the modulating RF signal, while the shot noise relatively decreases with the modulation depth $m$. In addition to the above are the wireless channel additive noise and thermal noise that are signal independent. Furthermore, the higher the spread-spectrum signal bandwidth, the higher the noise power. However, the higher bandwidth is better at suppressing multiuser interference. All these factors introduce a quite complex situation that is investigated in this chapter. 


\begin{tabular}{|c|c|c|c|c|}
\hline \multirow{2}{*}{$\begin{array}{l}\text { Base } \\
\text { Station }\end{array}$} & & \multirow{2}{*}{$\begin{array}{l}\text { ROF } \\
\text { Link }\end{array}$} & & \multirow{2}{*}{$\begin{array}{l}\text { Wireless } \\
\text { Channel }\end{array}$} \\
\hline & $s(t)$ & & $u(t)$ & \\
\hline
\end{tabular}

Figure 5.1: Block diagram showing a fiber based wireless CDMA system

\subsection{The Multiuser CDMA Signal}

Considering a direct spread CDMA system, the signal emanating from the BS $s(t)$, is a linear addition of multiple spread spectrum (SS) signals. Referring to Fig. 5.1, this combined signal $s(t)$ is given by (5.1).

$$
s(t)=\frac{1}{S_{\max }} \sum_{k=0}^{K-1} \sqrt{2 P_{k}} a_{k}(t) b_{k}(t) \cos \left(\omega_{c}(t)+\theta_{k}(t)\right)
$$

where $b_{k}(t)$ is the $k^{\text {th }}$ user baseband bipolar data, $a_{k}(t)$ is the $k^{\text {th }}$ pseudo-noise binary code with a chip rate of $R_{c}, \omega_{c}$ is the radian carrier frequency, $\theta_{k}(t)$ is the phase of the carrier associated with $k^{\text {th }}$ SS signal and $P_{k}$ is the power of the $k^{\text {th }}$ signal. $S_{\max }$ is the maximum value of $s(t)$ so that, $|s(t)| \leq 1$. The total number of users $K$, depends on a particular system. For example, in IS-95, $K$ is up to 64 per sector. However, in typical microcellular systems, $K$ is much smaller. All $K$ signals are synchronized at the base station with a common clock.

Let $u(t)$ be the output from the optical channel. In general, $u(t)$ can be written as in (5.2), where $F($.$) denotes a general non-linear function and n_{o p}(t)$ is the optical channel noise.

$$
u(t)=F(s(t))+n_{o p}(t)
$$

Initially, we like to do the analysis, only for the downlink, considering $F($.$) as$ a linear function and assuming that the signal processing techniques are able to successfully mitigate the nonlinear distortions. In this case, the combined channel (linearizing block plus the optical channel) is assumed linear. However, any impairment introduced by the additional signal processing blocks need to be addressed separately.

From Fig. 5.1, $r(t)$ is the received signal from the wireless channel. To better understand the noise and interference behavior we first assume an AWGN wireless 


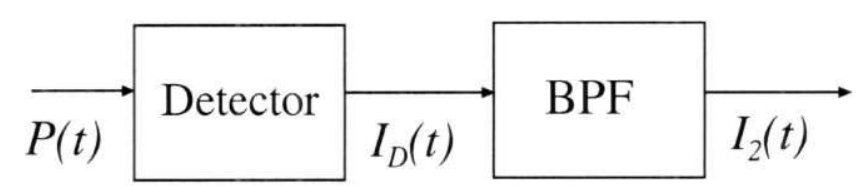

Figure 5.2: Block diagram showing detected and filtered currents

channel.

\subsection{Optical Channel Noise Mechanisms}

In this section, we derive expressions for the variances of optical link noise processes. These expressions are needed to evaluate the signal to noise ratio that is done in the next section. Although several noise mechanisms exist in a directly modulated ROF link, in this section only the shot, RIN and thermal are analyzed. Some other noise mechanisms such as the polarization mode noise and the mode hopping noise can be avoided with a careful design. These are not considered here.

Here, we assume that all connectors and splitters are ideal so that no distortion other than attenuation in the fiber occurs. We do not consider fiber chromatic dispersion. We also assume that the receiver has an electrical amplifier followed by an ideal band-pass filter of bandwidth $B$ centered at $f_{c}$. This arrangement is shown in Fig. 5.2. The transfer function of the receiver is $H(f)$ which, is shown in Fig. 5.3 .

\subsubsection{The Shot Noise}

In this section, an expression is derived for the shot noise variance. Considering a direct DSB intensity modulation at the laser diode for RF-Optical conversion, the instantaneous optical intensity output $P(t)$ from the laser in response to input electrical signal $s(t)$ is,

$$
P(t)=[1+m s(t)] P_{o}
$$

The above assumes a linear E/O conversion. Here $s(t),(|s(t)| \leq 1)$ is the spread spectrum electrical signal, $m$ is the optical modulation index and $P_{o}$ is the mean optical power.

The received optical signal at the receiver illuminates the photo-detector, which 
produces a detected current $I_{D}(t)$. The intensity of $I_{D}(t)$ is proportional to the instantaneous optical power $P(t)$. Now, let us define the 'coherent time' of the ROF link as the time within which, the instantaneous optical intensity of the link does not change significantly. This coherent time is much smaller than a period of the modulating radio frequency signal. Therefore, within the coherent time we can assume that the instantaneous optical intensity is constant. Note that even within this time, even if $P(t)$ is perfectly constant, the detector current is not constant. Rather, it is a shot noise process because, the arrival of light occurs as discrete units or 'photons'.

Therefore, $I_{D}(t)$ can be written as,

$$
I_{D}(t)=\sum_{k=-\infty}^{\infty} h_{D}\left(t-t_{k}\right)
$$

where, $h_{D}(t)$ is the impulse response of the photo detector. Since a PIN diode detector is assumed, the avalanche gain is unity. We shall first consider the shot noise induced by a constant optical power. Later, the noise added to the detected current due to a fluctuating optical power would be considered. The power spectral density (PSD) of the shot noise for a constant light input is given by [42],

$$
N_{\text {shot }}(f)=\left|H_{D}(f)\right|^{2} P_{o}+\left\langle I_{D}(t)\right\rangle^{2} \delta(f)
$$

where, $H_{D}(f)$ is the Fourier transform of $h_{D}(t)$ and $\left\langle I_{D}(t)\right\rangle$ is the mean of $I_{D}(t)$. From the above expression, we note that, if the impulse response of the detector is a dirac delta function for the given load impedance, then the shot noise has a white power spectral density. Typically, the capacitance of the detector is in the range of few picofarads [40]. Therefore, at wireless system sampling rates, the shot noise is white.

To consider the effect of filtering after detection, let the detected current $I_{D}(t)$ be filtered by a filter with a transfer function $H(f)$ to obtain an output current $I_{2}(t)$. Then, the mean of $I_{2}(t)$ is simply the convolution of the received signal with 


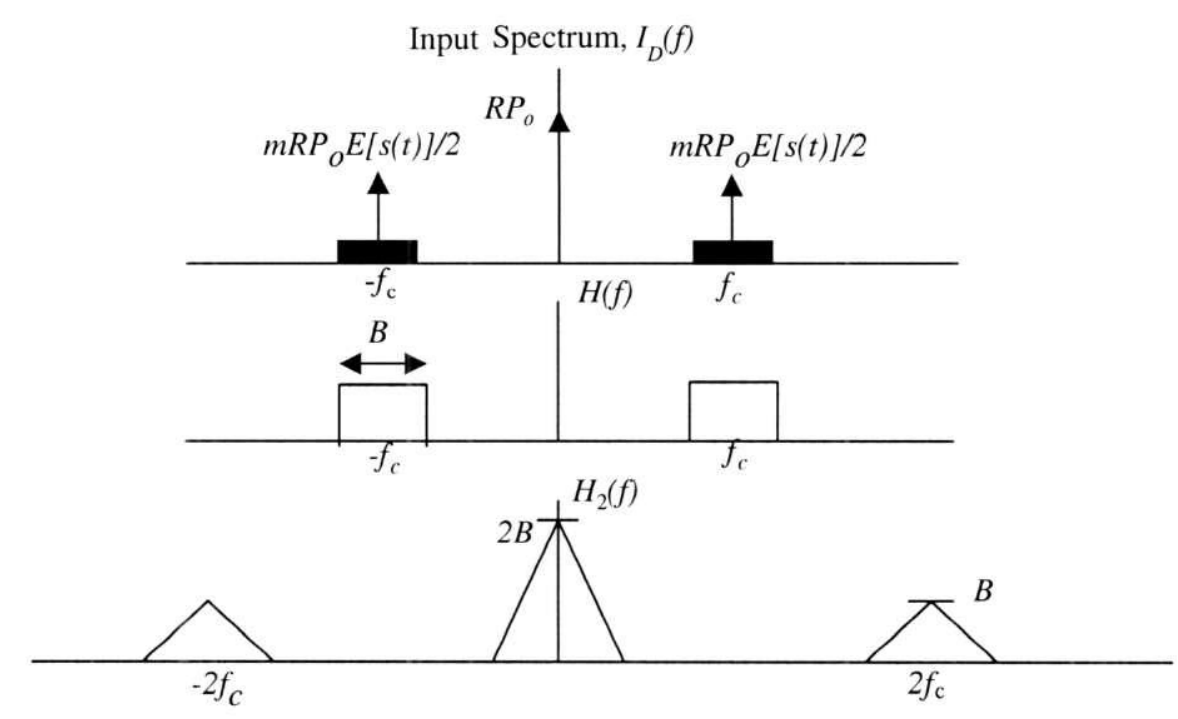

Figure 5.3: Down converted input spectrum and the first and second order frequency response of the receiver filter

the impulse response of the receiver filter $h(t)$.

$$
E\left[I_{2}(t)\right]=\int_{-\infty}^{\infty}\left\langle I_{D}(\tau)\right\rangle h(t-\tau) d \tau
$$

Now, consider the detector responsivity $\Re$, that is, the ratio between the detected current $I_{D}(t)$ and optical power $P(t)$. This is given by (5.7) for a PIN detector.

$$
\Re=\frac{\eta_{q} q}{h_{p} f_{o}}=\frac{\left\langle I_{D}\right\rangle}{P_{o}}=\frac{I_{D}(t)}{P(t)}
$$

Here, $q$ is the electron charge, $\eta_{q}$ is the quantum efficiency of the photo-detector, $h_{p}$ is the Planck's Constant $\left(6.626 \times 10^{-34}\right.$ Joules/Sec $)$ and $f_{o}$ is the optical carrier frequency. The right most part of this equation assumes $\Re$ does not change with $P(t)$.

Since $I_{D}(t)$ is, $P(t)$ multiplied by the detector responsivity $\Re$, (5.6) becomes,

$$
E\left[I_{2}(t)\right]=\Re \int_{-\infty}^{\infty} P(\tau) h(t-\tau) d \tau
$$

The variance of $I_{2}(t)$ after the filter is given by (5.9). The derivation of (5.9) is given in appendix $\mathrm{C}$ of [42] using a moment generating function of the photodiode. 
Note the appearance of $q$.

$$
\operatorname{Var}\left[I_{2}(t)\right]=\Re q \int_{-\infty}^{\infty} P(\tau) h^{2}(t-\tau) d \tau
$$

Note that both mean and variance of $I_{2}(t)$ are time varying quantities that are determined by linear filtering operations on $I_{D}(t)$. Typically, the bandwidth $B$ of the receiver filter is greater than the bandwidth of $s(t)$. Therefore, the received signal passes through the filter without any distortion. The filter, however, blocks the direct current term $\Re P_{o}$. Hence, the mean detected current depends on the sidebands only.

$$
E\left[I_{2}(t)\right]=\Re P_{o} m E[s(t)]=\left\langle I_{D}\right\rangle m E[s(t)]
$$

where, $E\left[s^{2}(t)\right]$ is the mean power of the transmitted signal. Since, $s(t)$ is given by (5.1), the mean power is just the summation of all $P_{k}$. That is,

$$
E\left[s^{2}(t)\right]=\left(\frac{1}{S_{\max }} \sum_{k=0}^{K-1} P_{k}\right) \leq 1
$$

If we carefully examine (5.9), the variance of $I_{2}(t)$ is equal to the signal $P(t)$ filtered through a hypothetical filter that has an impulse response $h_{2}(t)=h^{2}(t)$. The frequency responses of both $H(f)$ and $H_{2}(f)$ are shown in Fig 5.3. $H_{2}(f)$ is obtained by performing Fourier transform on $h_{2}(t)$. From the transfer function $H_{2}(f)$, it is obvious that only the DC term $\Re P_{o}$ passes through this filter. Since $H_{2}(f)$ has no gain around $f_{c}$, both side bands are attenuated. Therefore, the shot noise variance after the filter is given by,

$$
\operatorname{Var}\left[I_{2}(t)\right]=\left\langle I_{\text {shot }}^{2}\right\rangle=2 q \Re P_{o} B=2 q\left\langle I_{D}\right\rangle B
$$

This expression for the variance of the shot noise is widely used in the literature. However, it is important to remember that this is only valid when the receiver has an ideal bandpass filter and the detector responsivity $\Re$ is constant. 


\subsubsection{The Relative Intensity Noise}

The laser does not produce light that is stable in intensity. The basic physical mechanism of a laser is amplification by stimulated emission, which is random in nature. This randomness introduces a noise that increases with the optical power. The noise due to multiple optical reflections (Interferometric noise) and Brillouin scattering also increase with optical power. All these noise processes can be grouped together as relative intensity noise (RIN). The expression for the modulated optical signal considering RIN is,

$$
P(t)=[1+m s(t)]\left[P_{o}+\Delta P(t)\right]
$$

where $\Delta P$ is the instantaneous fluctuation term due to relative intensity noise.

To obtain an expression of the detector current with RIN, the filter output current is the convolution with filter impulse response $h(t)$.

$$
I_{2}(t)=\Re \int_{-\infty}^{\infty} P(\tau) h(t-\tau) d \tau
$$

The primary shot noise process $I_{2}(t)$ is a doubly stochastic Poisson process in the presence of the relative intensity noise. The mean and variance of this doubly stochastic output process are determined by the generalized Campbell theorem as shown in [42].

The average of $I_{2}(t)$ is the same as the previous average in (5.10) since, the $\Delta P(t)$ is a zero mean process.

$$
E\left[I_{2}(t)\right]=E\left\{\Re \int_{-\infty}^{\infty}[1+m s(\tau)]\left[P_{o}+\Delta P(\tau)\right] h(t-\tau) d \tau\right\}=I_{D} m s(t)
$$

The variance of $I_{2}(t)$ is given by,

$$
\operatorname{Var}\left[I_{2}(t)\right]=E\left[I_{2}^{2}(t)\right]-\left\{E\left[I_{2}(t)\right]\right\}^{2}
$$


From (5.8) and (5.9), this simplifies to,

$$
\operatorname{Var}\left[I_{2}(t)\right]=E\left\{\Re q \int_{-\infty}^{\infty} P(\tau) h^{2}(t-\tau) d \tau\right\}+\operatorname{Var}\left\{\Re \int_{-\infty}^{\infty} P(\tau) h(t-\tau) d \tau\right\}
$$

The first term in (5.17) is the variance of the shot noise due to the constant optical input same as 5.9), which is already considered in section 5.2.1. The second term is the variance of the relative intensity noise after the receiver filter. This is re-written as,

$$
\left\langle I_{R I N}^{2}\right\rangle=\operatorname{Var}\left\{\Re \int_{-\infty}^{\infty} P(\tau) h(t-\tau) d \tau\right\}
$$

Since variance is the expectation of the second order term, and $P(t)=[1+$ $m s(t)]\left[P_{o}+\Delta P(t)\right]$ this simplifies to,

$$
\left\langle I_{R I N}^{2}\right\rangle=E\left[\left\{\Re \int_{-\infty}^{\infty}[1+m s(\tau)] \Delta P(\tau) h(t-\tau) d \tau\right\}^{2}\right]
$$

Now, we define a new filter $h_{3}(\tau)=[1+m s(t-\tau)] h(\tau)$, such that,

$$
\left\langle I_{R I N}^{2}\right\rangle=\left[\left\{\Re \int_{-\infty}^{\infty} \Delta P(\tau) h_{3}(t-\tau) d \tau\right\}^{2}\right]
$$

When this expression is converted to frequency domain,

$$
\left\langle I_{R I N}^{2}\right\rangle=\Re^{2} \int_{-\infty}^{\infty} N_{R I N}(f)\left|H_{3}(f)\right|^{2} d f
$$

Here, $N_{R I N}(f)$ is the double sided power spectral density of the relative intensity noise. At frequencies of interest for analog optical transmission this has a constant spectrum [40].

From the conservation of power, the power confined in the spectrum of $\left|H_{3}(f)\right|^{2}$ is same as the average square value of the term $[1+m s(t)]$ for an ideal filter $H(f)$. This is independent of the spectral shape of $s(t)$. Therefore, the noise power due to 
RIN is given as,

$$
\left\langle I_{R I N}^{2}\right\rangle=2 \Re^{2} N_{R I N} B\left[1+m^{2}\left\langle s^{2}(t)\right\rangle\right]
$$

The expression we obtained here is different from the widely used expression for the variance of the RIN. Many authors including Way, have omitted the second term $m^{2}\left\langle s^{2}(t)\right\rangle$. This is acceptable because, most of the time $m$ is in the range of 0.1 and $s(t)<<1$ so that, this term is insignificant. However, with higher values of $m$ and $s(t)$ this term is not negligible. We include this term because with nonlinearity compensation schemes, $m$ can be higher. Furthermore, the expression in (5.22) better explains, empirical results obtained in Chapter 3.

\subsubsection{The Thermal Noise}

Thermal noise arises due to the random fluctuation of electrons, which is a function of absolute temperature. Any resistive element introduces thermal noise. In ROF links, the resistance in the photodiode as well as in the amplifier that follows it adds thermal noise. The equivalent current noise source has a double-sided power spectral density. The variance of this noise multiplied by the amplifier noise factor $F$ is given below where, $K_{b}$ is the Boltzmann's constant $\left(1.38054 \times 10^{-23} \mathrm{Ws} / \mathrm{K}\right)$, and $T_{o}$ is the absolute temperature in Kelvin and $R_{L}$ is the load resistance.

$$
\left\langle I_{t h}^{2}\right\rangle=4 F K_{B} T_{o} B / R_{L}
$$

\subsubsection{The Dark Current Noise}

An additional noise source in the photodiode is a dark current $I_{\text {dark }}$. This is generated even in the absence of optical power as the name implies and has a similar expression to shot noise. The noise power associated with this is given by [40],

$$
\left\langle I_{\text {dark }}^{2}\right\rangle=2 q I_{\text {dark }} B
$$




\subsection{The Signal to Noise Ratio}

The complete signal to noise ratio of the ROF link considering all these noise processes is given below with an understanding that $I_{D}=\left\langle I_{D}\right\rangle$.

$$
S N R=\frac{m^{2} I_{D}^{2} E\left[s^{2}(t)\right]}{2 q B\left(I_{D}+I_{\text {dark }}\right)+2 \Re^{2} N_{R I N} B\left[1+m^{2} E\left[s^{2}(t)\right]\right]+4 F K_{B} T_{o} B / R_{L}}
$$

The dark current $I_{\text {dark }}$ is typically on the order of nano-Amps. The detected current $I_{D}$ is typically in milli-Amps range since the optical power in this applications is in $\mathrm{mW}$ range and the responsivity $\Re$ lies between zero and one $\mathrm{mA} / \mathrm{mW}$. Therefore, the dark current term is neglected without any loss in accuracy. Hence, the modified signal to noise ratio due to optical link noises is given by,

$$
S N R=\frac{m^{2} I_{D}^{2} E\left[s^{2}(t)\right]}{2 q B I_{D}+2 \Re^{2} N_{R I N} B\left[1+m^{2} E\left[s^{2}(t)\right]\right]+4 F K_{B} T_{o} B / R_{L}}
$$

Typically, a RIN value is specified for a given laser diode in $\mathrm{dB} / \mathrm{Hz}$, for example $-155 \mathrm{~dB} / \mathrm{Hz}$. This RIN value is related to the double-sided power spectral density $N_{R I N}$ by,

$$
R I N(d B / H z)=\left\langle 2 N_{R I N}\right\rangle / P_{o}^{2}
$$

Substituting this relationship and simplifying yields the expression for SNR given below, where the $(R I N)_{l i n}$ is in the linear scale. $\left((R I N)_{l i n}=10^{\frac{R I N(d B / H z)}{10}}\right)$.

$$
S N R=\frac{m^{2} I_{D}^{2} E\left[s^{2}(t)\right]}{\left(2 q I_{D}+(R I N)_{l i n} I_{D}^{2}\left[1+m^{2} E\left[s^{2}(t)\right]\right]+4 F K_{B} T_{o} / R_{L}\right) B}
$$

\subsubsection{Quantitative Discussion}

There are three noise terms involved in the expression given. Namely shot, RIN and thermal noises. Thermal noise has a constant variance and depends on the receiver resistance only. This has a white spectrum. The variance of the shot noise is linearly proportional to mean optical power in the fiber (see (5.12)). Although the instantaneous optical power in the fiber fluctuates due to RF intensity modulation, the mean optical power does not change unless the DC bias current is changed. Therefore, the shot noise does not change with RF power and is constant for a given 
modulation depth $m$. However, the RIN changes with RF signal level. This is seen from the expression in (5.22). This is also logical because, the RIN is proportional to the square of the optical power. Since, the instantaneous optical power in the fiber fluctuates at radio frequency, the square of it increases with RF signal level depending on $m$.

The following additional points are observed from the expression for signal to noise ratio:

1. The higher the bandwidth $B$ of $s(t)$, the lower the SNR. The reason is because the wider noise bandwidth in the optical link collects more noise. However, at the portable receiver when the SS signal is despread wider bandwidth means more processing gain. Therefore, there is an optimal bandwidth $B$ which, depends on number of users as well as ROF link characteristics.

2. The higher modulation index $m$ yields better SNR. This is because more power is contained in the sidebands compared to the unmodulated carrier. However, nonlinear effects limit $m$ to a lower value $(m<0.3)$. Nevertheless, with our proposed nonlinearity compensation schemes $m$ can be made higher. Therefore, nonlinearity compensation helps to improve signal to noise ratio of the link too.

If we assume the thermal noise at the receiver amplifier is made small due to an improved design [9], then (5.28) becomes,

$$
S N R=\frac{m^{2} I_{D} E\left[s^{2}(t)\right]}{\left(2 q+(R I N)_{l i n} I_{D}\left[1+m^{2} E\left[s^{2}(t)\right]\right]\right) B}
$$

Note that, in this case Fig. 5.4 would have a lower thermal noise. From (5.29) we deduce that,

1. In the shot noise limited case $S N R=m^{2} I_{D} E\left[s^{2}(t)\right] / 2 q B$. That is, SNR increases with mean detected current $I_{D}$. Mean detected current is proportional to mean optical power $P_{o}$. However, large $P_{o}$ means low $m$ assuming the total optical power is limited. Therefore, there is an optimum $m$ in the shot noise limited case. 


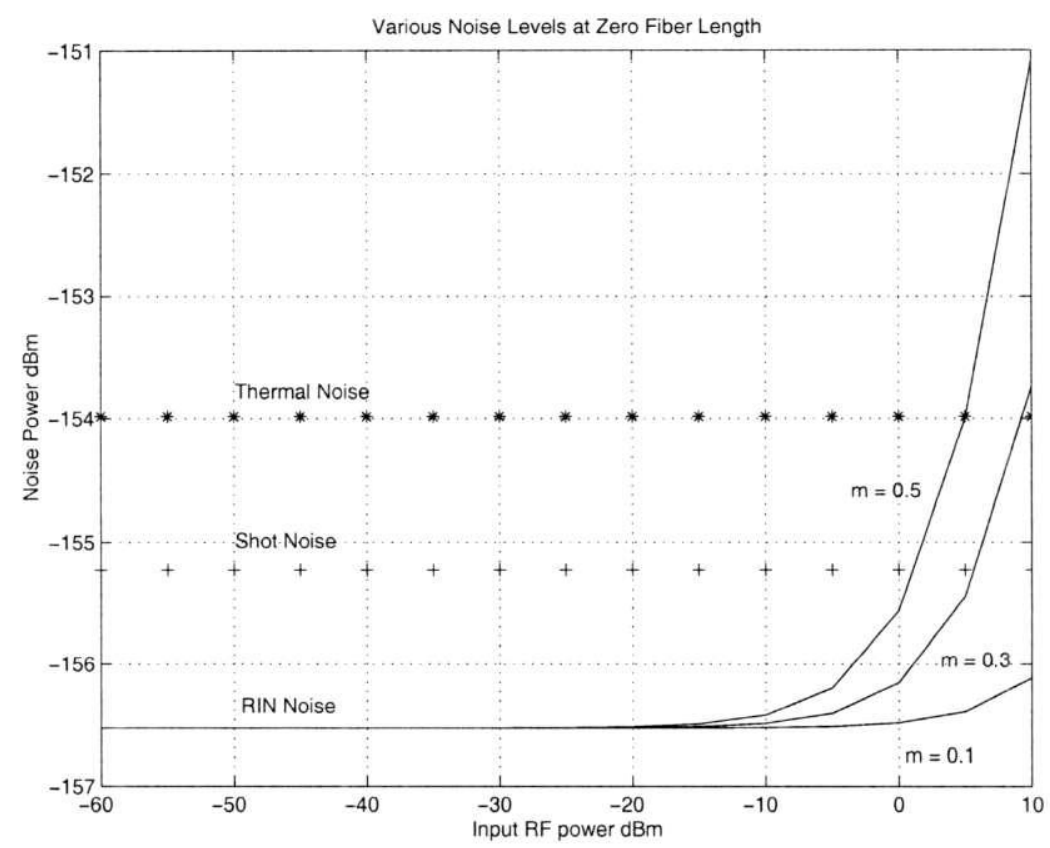

Figure 5.4: Various noise powers of the ROF link as a function of input RF power

2. In the RIN limited case, $S N R=m^{2} E\left[s^{2}(t)\right] /(R I N)_{l i n}\left[1+m^{2} E\left[s^{2}(t)\right] B \approx\right.$ $m^{2} E\left[s^{2}(t)\right] /(R I N)_{l i n} B$. That is the SNR is independent to mean optical power and increases with the RF power. However, when the RF power is large enough $\left(m^{2} E\left[s^{2}(t)\right]>1\right)$, the SNR saturates.

Note that, in the expression in (5.28) the multiuser spread spectrum signal $s(t)$ is treated as a single quantity. This has to be suitable scaled in order to quantify the signal to noise ratio for each user. This is done easily by dividing by $K$ when, all $K$ users have the same power.

\subsubsection{Numerical Results}

To understand the relative importance of each of these noise mechanisms, let us plug in some numerical values. Let us assume, for a single mode laser the specified RIN is $-156.5 \mathrm{~dB} / \mathrm{Hz}$ (typically -140 to $-160 \mathrm{~dB} / \mathrm{Hz}$ ), the filter bandwidth $\mathrm{B}$ is 1.25 $\mathrm{MHz}$ (IS-95), the responsivity of the PIN detector is $0.75 \mathrm{~A} / \mathrm{W}$ and the dark current is $6 \mathrm{nA}$. The mean optical power in the fiber is $1 \mathrm{~mW}$. The load resistance is $50 \Omega$ The receiver amplifier has a noise figure of $3 \mathrm{~dB}$. Let us determine the noise and SNR at the receiver as a function of transmitted power $\left(P_{R F}(t)=\left\langle s^{2}(t) / R_{L}\right\rangle\right)$ for 


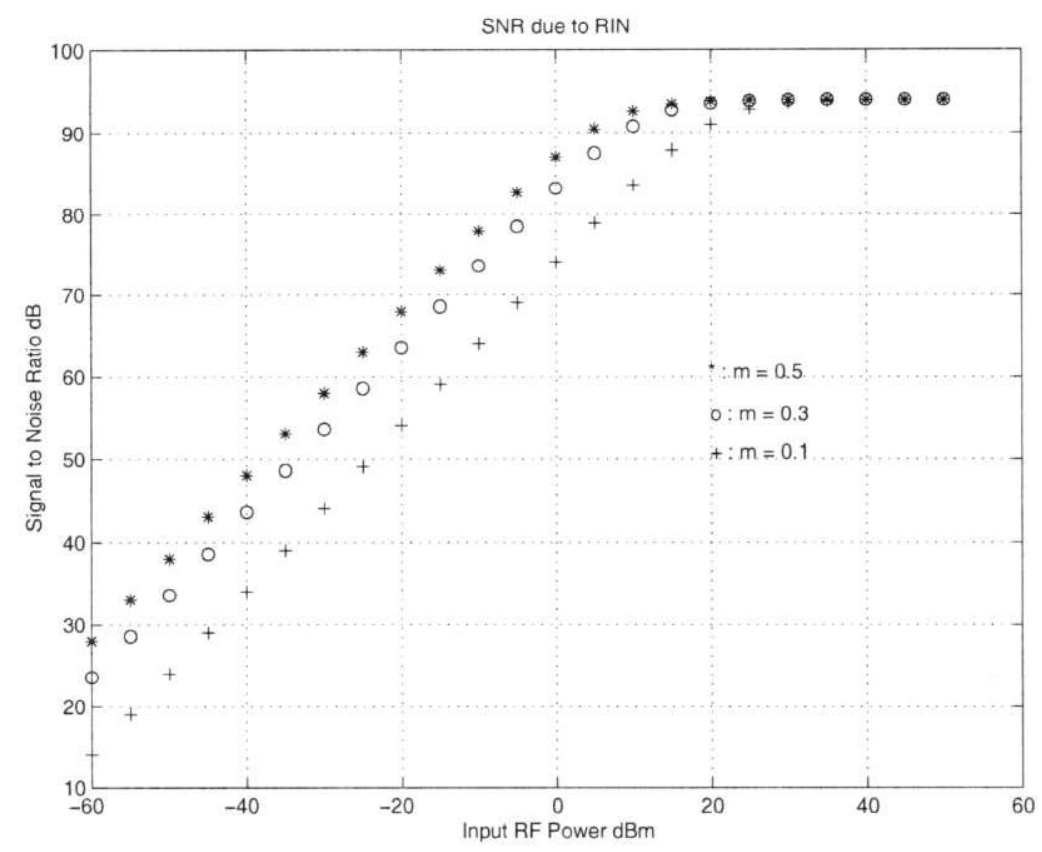

Figure 5.5: Signal to noise ratio due the relative intensity noise only

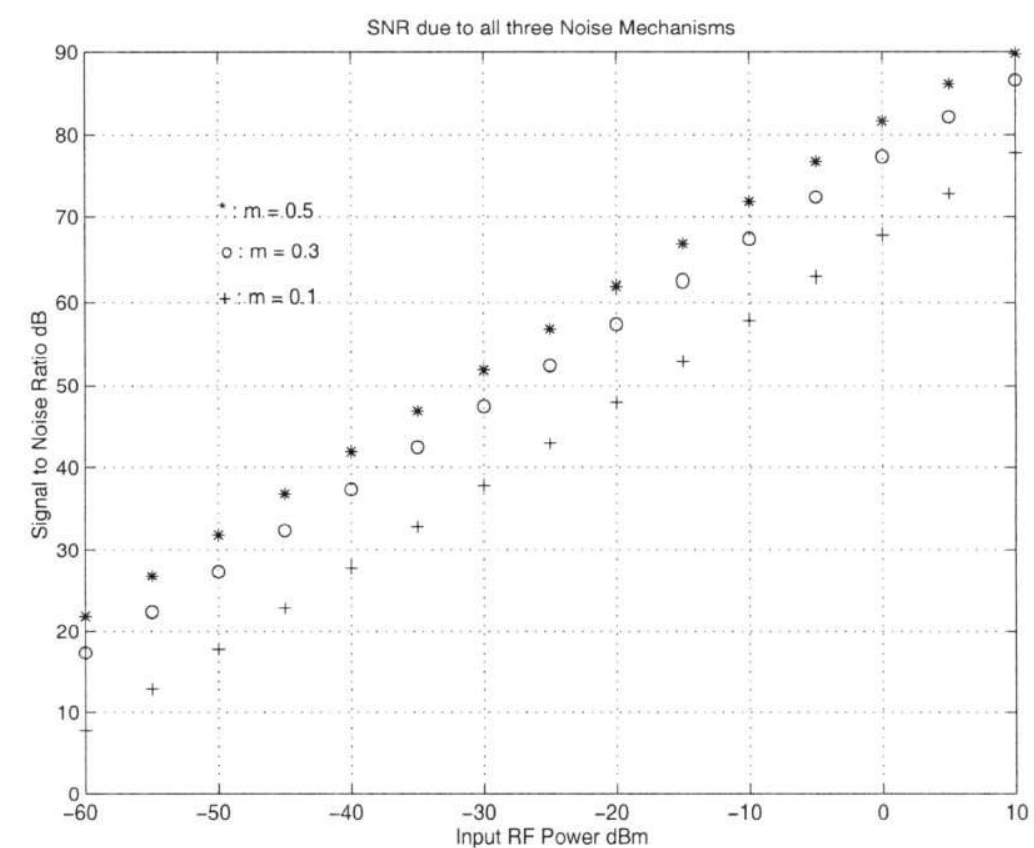

Figure 5.6: Total signal to noise ratio of the ROF link

the modulation index $m=0.1,0.3$ and 0.5 . A zero fiber length is assumed that is there is no attenuation in the optical power.

The noise plot is shown in Fig. 5.4, where the shot and thermal noise are constant. The RIN is constant up to the point where the input RF power is -10 
$\mathrm{dBm}$. Above $-10 \mathrm{dBm}$ it starts to increase. The rate of increase depends on the modulation depth $m$. This theoretical graph agrees with what we experimentally observed in Fig. 3.6 in Chapter 3.

Fig. 5.5 shows the signal to noise ratio due to the RIN only. As expected, the SNR value first linearly increases with $P_{R F}$. However, when $m^{2} E\left[s^{2}(t)\right]>1$, it saturates. To show this, the input power is increased up to $50 \mathrm{dBm}$, which is much higher than typical values. Fig. 5.6 shows the overall signal to noise ratio. It does not seem to saturate hard because when all three-noise terms are added the weight of RIN is low and, the saturation would come above $10 \mathrm{dBm}$ input RF power.

\subsection{Matched Filter Detection}

In the downlink, single user detection schemes are adequate because of the small cell size. This implies few interfering users and less near far problem. Because of this, even simple matched filter detection would give good performance with adequate spreading gain.

Let us first consider an additive white Gaussian noise (AWGN) wireless channel. In this case the received signal $r(t)$ is just the scaled version of $s(t)$ where $s(t)$ is defined in (5.1).

$$
r(t)=\frac{G_{o p t} G_{w l}}{S_{\max } L_{w l} L_{o p t}} \sum_{k=0}^{K-1} \sqrt{2 P_{k}}(t) a_{k}(t) b_{k}(t) \cos \left[\omega_{c} t+\theta_{k}(t)\right]+n(t)
$$

where, $n(t)$ is the addition of optical and wireless channel noises, $G_{\text {opt }}$ is the optical post amplifier gain, $G_{w l}$ is the portable receiver post amplifier gain, $L_{w l}$ is the wireless channel loss and $L_{\text {opt }}$ is the optical channel loss.

Neglecting the scaling that can be easily compensated for, $r(t)$ is written as,

$$
r(t)=\sum_{k=0}^{K-1} \sqrt{2 P_{k}}(t) a_{k}(t) b_{k}(t) \cos \left[\omega_{c} t+\theta_{k}(t)\right]+n(t)
$$

Without the loss of generality, let the desired user be assumed as the first user Then $r(t)$ is multiplied with the PN sequence $a_{1}$ and integrated over one bit period 
$T_{b}$ of say, the first bit. The output $Z_{1}$ is given by,

$$
Z_{1}=\int_{0}^{T_{b}} r(t) a_{1}(t) \cos \left(\omega_{c} t\right) d(t)
$$

This is expressed as,

$$
Z_{1}=I_{1}+\eta+\zeta
$$

where, $I_{1}$ is the desired user contribution to the decision statistics, $\zeta$ is the multiple access interference (MAI) from all co-channel users and $\eta$ is the noise contribution. From [3] $I_{1}$ is written as,

$$
I_{1}=\sqrt{\left(P_{1} / 2\right)} b_{1,1} T_{b}
$$

where, $b_{1,1}$ is the first bit of the first user and $P_{1}$ is the power of the first user.

The noise term $\eta$ is given by,

$$
\eta=\int_{0}^{T_{b}} n(t) a_{1}(t) \cos \left(\omega_{c} t\right) d t
$$

\subsubsection{Comparison of Optical and Wireless Channel Noise}

Further analysis on the noise term $n(t)$ is necessary for a quantitative understanding of the relevant importance of each noise mechanism. $n(t)$ consists of optical channel noise terms $n_{\text {opt }}(t)$ as well as wireless channel noise $n_{w l}(t)$. The signal is weak at the optical receiver where $n_{\text {opt }}(t)$ is added to it. $n_{\text {opt }}(t)$ is amplified by optical post $\operatorname{amplifier}\left(G_{o p t}\right)$ and then undergoes wireless channel loss $L_{w l}$. Again, at the portable receiver, $n_{w l}(t)$ is added to the signal.

$$
n(t)=\left(\frac{n_{o p t}(t) G_{o p t}}{L_{w l}}+n_{w l}(t)\right) G_{w l}
$$

From (5.36), depending on the values of the gain parameters, wireless or optical channel noise may dominate the overall SNR. The optical channel noise is composed of relative intensity noise (RIN), shot noise and thermal noise. These noise mechanisms have been discussed previously. The wireless channel noise composed of man made noise plus thermal noise.

Typically $n_{\text {opt }}$ and $n_{w l}$ are in the same order. The $G_{o p t}$ is a constant while the 
average of $L_{w l}$ increases with distance. Therefore, when the portable unit is close to the RAP, that is $L_{w l}<G_{o p t}, n_{\text {opt }}$ is the dominating noise. On the other hand, when the portable is far away from the RAP, where $L_{w l}>G_{o p t}, n_{w l}$ is the dominating noise.

Let us examine the spectrum of each noise process. The relative intensity noise is a zero mean stochastic process. Its power spectral density depends on the design of the laser and its pumping conditions. At frequencies of interest for analog optical transmission the (double-sided) spectral density can be considered to be constant [40]. If the light input is constant, the primary shot noise process is a stochastic Poisson process. If the photo-detector has a short impulse response compared to other time factors in the system, then the shot noise is white noise [42]. It is well known that thermal noise is white. The (man made) impulsive wireless channel noise is also a white Gaussian noise. Since all these noise processes are uncorrelated with each other, their power spectral densities can be added. Assume a two-sided spectrum of level $N_{o} / 2$. for the combined noise, then the mean and variance of $\eta$ are,

$$
\begin{gathered}
E[\eta]=\int_{o}^{T_{b}} E[n(t)] a_{1}(t) \cos \left(\omega_{c} t\right) d t=0 \\
E\left[(\eta-E[\eta])^{2}\right]=\sigma_{\eta}^{2}=N_{o} T_{b} / 4
\end{gathered}
$$

Equation (5.38) is true for $\omega_{c}>>2 / T_{b}[3]$.

\subsubsection{The Bit Error Rate}

Because of the discrete photons, the basic statistical model in optical communication is the Poisson process. Despite this, the Gaussian density is often used to obtain probability of error expressions and it is usually accurate [42]. Furthermore, in analog optical links, when noise depends on the RF signal, which is Gaussian distributed, the optical noise is also Gaussian. Therefore, $\eta$ is modeled as a zero mean Gaussian process.

The component $\zeta$ in the expression for $Z_{1}$ represents the contribution of MAI in 


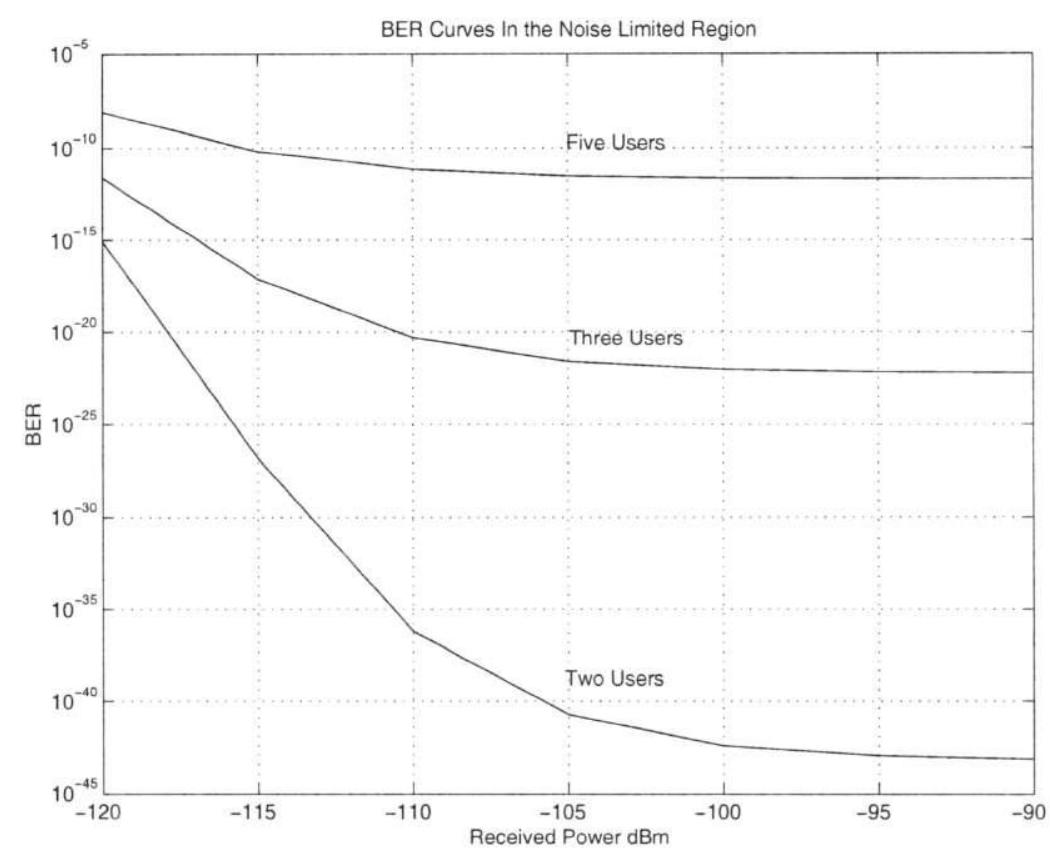

Figure 5.7: BER curves of a fiber wireless CDMA system with matched filter detection in the noise limited region.

the decision statistic. $\zeta$ is the summation of $K-1$ terms of $I_{k}$.

$$
\zeta=\sum_{k=2}^{K} I_{k}
$$

According to the central limit theorem, $\zeta$ is a zero mean Gaussian random variable as $K-1$ gets large. Therefore, the variance of $\zeta$ is given by,

$$
\sigma_{\varsigma}^{2}=\frac{N_{c} T_{c}^{2}}{6} \sum_{k=2}^{K} P_{k}
$$

where $N_{c}$ is the length of the spreading code and $T_{c}$ is the chip time. Now, the decision statistic $Z_{1}$ is modeled as a Gaussian random variable with mean $I_{0}$ and variance $\sigma_{\varsigma}^{2}+\sigma_{\eta}^{2}$,

$$
\sigma_{\varsigma}^{2}+\sigma_{\eta}^{2}=\frac{N_{c} T_{c}^{2}}{6} \sum_{k=2}^{K} P_{k}+\frac{N_{0} T_{b}}{4}
$$

Because of the Gaussian assumption, the average bit error rate (BER) probabil- 
ity is given by

$$
\begin{gathered}
B E R=Q\left(\frac{\sqrt{\frac{P_{0}}{2}} T_{b}}{\sqrt{\frac{N_{c} T_{c}^{2}}{6} \sum_{k=2}^{K} P_{k}+\frac{N_{0} T_{b}}{4}}}\right) \\
B E R=Q\left(1 / \sqrt{\frac{1}{3 N_{c}} \sum_{k=2}^{K} \frac{P_{k}}{P_{0}}+\frac{N_{0}}{2 T_{b} P_{0}}}\right)
\end{gathered}
$$

Assuming perfect power control, where $P_{k}=P_{o} \forall k=1,2,3, \ldots . K$, the BER is,

$$
B E R=Q\left(\frac{1}{\sqrt{\frac{K-1}{3 N_{c}}+\frac{N_{0}}{2 T_{b} P_{0}}}}\right)
$$

In this expression, the BER is evaluated assuming the average value for the variance of MAI. Typically, the above Gaussian approximation is very close to the values obtained by simulation when the number of users are above 15 [3]. When it is less than 15 , then the BER calculation is little more pessimistic. However, here we used Gaussian assumption just to get a feel of the noise performance.

The BER curves generated from this formula are shown in Fig. 5.7. Code length $N_{c}$ of 64 and bit rate $R_{c}$ of $19.2 \mathrm{kbps}$ are used. The effect of combined noise is seen in the BER performance with two or three users. As the number of users increases, the BER curve flattens because, with matched filter detectors, a CDMA system is interference limited when the interference power exceeds the noise power. Here, the overall BER performance is very good because of large $N_{c}$ and few users. 


\section{Chapter 6}

\section{A Joint Compensation to the Fiber-Wireless Channel}

The first part of this dissertation is dedicated for understanding the characteristics of the ROF link on an element by element basis, using both hardware measurements and theory. Then, baseband signal processing techniques are investigated for performance improvement assuming the wireless channel is an AWGN channel. In high speed wireless links, however, multipath dispersion of the wireless channel is very significant. Therefore, in this and subsequent chapters, a multipath wireless channel is considered in series with the nonlinear optical channel and, a unified approach is taken to investigate the effect of this combination.

In this chapter, first, key properties of indoor wireless channels are briefly discussed. Then, Wiener/Hammerstein type nonlinear systems and their identification, either parametric or structural, is reviewed. The fiber-wireless channel is modeled as a Wiener system and as a Hammerstein system in the uplink and in the downlink respectively. A PN sequence based channel estimation algorithm is then mathematically derived. The algorithm identifies the linear and nonlinear parts separately. Finally, simulation results are given to verify the derivations.

\subsection{The Indoor Wireless Channel}

In this section, the multipath fading nature of the indoor wireless channel is discussed. Indoor wireless channels are considered since, the highest demand for wide- 
band access is indoors. Also, the limited RF power at the RAP of a fiber based wireless system is more suitable to serve an indoor microcell.

In indoor wireless environments, the distance covered is much smaller and the variability of the environment is much greater for a given transmitter-receiver separation. This makes characterization of an indoor wireless channel relatively difficult. However, there are some key factors that generally apply around $1.5 \mathrm{GHz}[49]$ :

1. The indoor channel is quasi-static or slowly time varying.

2. If there is no line of sight path, the maximum multipath delay spread is 100 ns to $200 \mathrm{~ns}$ within the rooms of a building and $300 \mathrm{~ns}$ in hallways.

3. The measured rms delay spread within rooms (typically with a line of sight) has a median of $25 \mathrm{~ns}$ and a maximum value of $50 \mathrm{~ns}$.

The delay spread is an important measure because, it affects the coherence bandwidth. The coherence bandwidth is a statistical measure of the bandwidth over which, the channel is considered flat (equal gain and linear phase). Two sinusoids with frequency separation greater than the coherence bandwidth are effected quite differently by the channel. The coherence bandwidth $\left(B_{c}\right)$ and the rms delay spread $\left(\sigma_{\tau}\right)$ of the channel have an inverse relationship.

If the coherence bandwidth is defined as the bandwidth over which the frequency correlation function is above $90 \%$, then it is expressed as, $B_{c} \approx 1 /\left(50 \sigma_{\tau}\right)$ [3]. On the other hand, if the coherence bandwidth is defined as the bandwidth over which the frequency correlation function is above $50 \%$, then it is expressed as, $B_{c} \approx 1 /\left(5 \sigma_{\tau}\right)$ [3]. The former relationship gives a more pessimistic measure while the latter is too optimistic. A realistic estimate will be somewhere in between these two.

A simple calculation shows that, if the delay spread is $25 \mathrm{~ns}$ then, assuming a $90 \%$ correlation, $B_{c}=800 \mathrm{kHz}$. This decreases to $400 \mathrm{kHz}$ if the delay spread increases to $50 \mathrm{~ns}$. If we assume $50 \%$ correlation then these values are $8 \mathrm{MHz}$ and $4 \mathrm{MHz}$ respectively. From the foregoing, it is obvious that, frequency selective fading and intersymbol interference (ISI) are inevitable in typical indoor wireless high speed systems. These factors come into play when the chip or bit rate is even a few Mbps. 


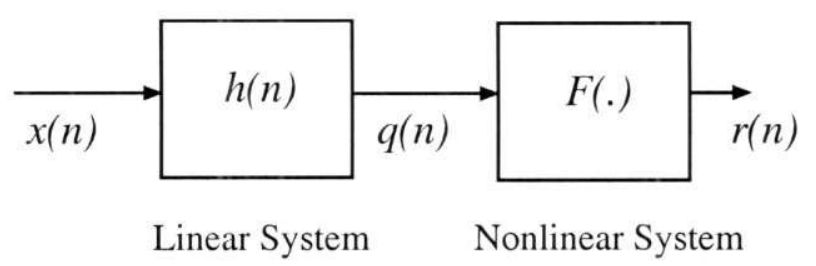

Figure 6.1: Block diagram of a Wiener system

\subsubsection{A Tapped Delay Line Model}

The multipath channel is modeled by an equivalent discrete-time transversal filter as shown in [50]. This facilitates the mathematical analysis that follows. It is more convenient to define a single impulse response $h(n)$ that includes the impulse responses of the transmit filter, wireless channel and the receive filter ${ }^{1}$. Since this $h(n)$ consists of individual tap weights, it is written as, $\left\{h_{i}(n) ; 0 \leq i \leq L\right\}$. The time-span of this filter is $L T_{s}$ seconds, assuming there are $(L+1)$ taps and a sampling rate of $1 / T_{s}$. Therefore, when $x(n)$ is transmitted, the received signal $q(n)$ is written as,

$$
q(n)=\sum_{i=0}^{L} h_{i} x(n-i)+\eta(n)
$$

where, $\eta(n)$ is a white Gaussian noise sequence.

The Doppler shift is very small for indoor channels [49], because the user is either stationary or at most walking. Therefore, the time domain representation of the Doppler spread, which is the coherence time of the channel, is much larger than the symbol period. In other words channel variation is much slower than the baseband signaling rate (quasi static channel). Therefore, the channel can be assumed stationary within a data frame duration.

\subsection{The Wiener/Hammerstein System Model}

A memoryless, mild nonlinearity can be described by a Taylor series which, is just a power series expansion. When memory is present, the Volterra series representation

\footnotetext{
${ }^{1}$ This includes a noise whitening filter as well, as described in [50]
} 


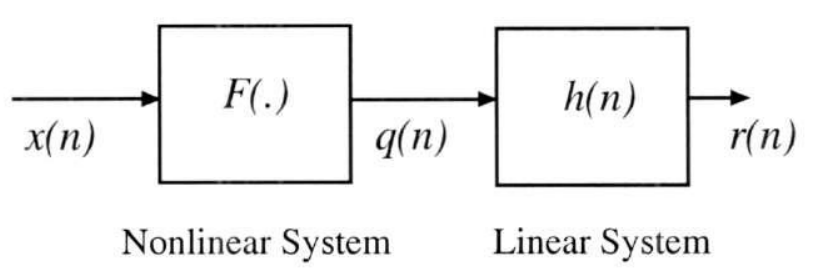

Figure 6.2: Block diagram of a Hammerstein system

is widely used. Just as an impulse response completely describes a linear system, the Volterra kernels completely describe a nonlinear system. However, there are two major drawbacks with the Volterra series representation [51]:

Convergence Many types of commonly encountered nonlinear systems such as saturating elements generally cannot be characterized by a Volterra series that converges for all inputs.

Measurement Measurement of Volterra kernels are possible only if the contribution of each Volterra operator is separated from the total system response which, is usually difficult.

Different models have been developed to circumvent this problem. In $1949 \mathrm{~N}$. Wiener investigated a new way of system identification using Gaussian inputs [28]. He derived a set of orthogonal functions from the Volterra kernels for Gaussian inputs that are called the G-functions. These G-functions greatly eased nonlinear system identification. Wiener showed that, for Gaussian inputs, any BIBO stable nonlinear system with finite memory can be modeled as a Wiener system which, consists of linear dynamic functions followed by static nonlinear functions. Similarly, modeling systems with a static nonlinearity followed by linear system is referred to as Hammerstein systems [52]. These are shown in Figures 6.1 and 6.2 respectively. It can be shown that Volterra systems are only a subset of Wiener systems [51] Since the Wiener functions provide an orthogonal basis, in theory the contribution of each basis function can be individually determined.

It should be pointed out that the Wiener system is defined only for Gaussian inputs. In practice however, inputs are not always Gaussian. Fortunately, modern spread spectrum communication systems use pseudo noise (PN) sequences which, 
behave like Gaussian noise in many aspects, but are easy to generate and analyze [29]. In this thesis use is made of these properties. The linear dynamic wireless channel followed by the static nonlinear ROF link is modeled as a Wiener system with a PN sequence input. If the order is reversed then, it is modeled as a Hammerstein system.

\subsection{Nonlinear System Identification}

We first review the identification of nonlinear systems. There are two steps in the nonlinear system identification: identifying the structure or the architecture of the unknown system and, identifying the parameters that characterize a particular structure.

\subsubsection{Structure Identification}

Structure identification is much more difficult than parameter identification. Several methods have been proposed for structure identification from input output data [53]. Volterra, Bilinear, Hammerstein and Wiener are some popular nonlinear system structures.

Billings et al. [54] have published a series of papers on structure identification. By expanding the system output in terms of the past inputs and outputs using a 'Nonlinear ARMA model with eXogenous inputs (NARMAX)' is considered in. They have derived a new orthogonal parameter estimation algorithm for this NARMAX model. The model starts with a large number of terms. Later, the contribution of each parameter is estimated and less significant terms are removed.

Fortunately, the structure of a communication channel is generally known. Thus, extensive structure identification schemes are not of our concern. On the other hand, structure identification is a key issue in other areas such as biology or controls.

\subsubsection{Parameter Identification}

Once the structure of the nonlinear system is determined, the parameters or the coefficients of the system are found. This can be done either through extensive 
measurements or by observing the output with a known input. We focus on the latter approach.

Input output correlation functions are extensively used for parameter identification. Billings et. al. [55] have shown that the input output correlation properties can be used to identify both the linear and nonlinear parts of the Wiener/Hammerstein systems. The same correlation properties can also be used to identify the system structure. Originally the results were for Gaussian inputs, but later they extended the work to include non Gaussian inputs such as pseudorandom inputs [30].

In another work [56], it has been shown that when a linear LMS adaptive filter is trained to model a Wiener system, it identifies the linear portion of the Wiener system to within a scale factor. The scale factor depends on the unknown nonlinearity and number of training samples. The same authors later extended the work to consider a combined Wiener/Hammerstein (WH) system. Drawbacks in this approach is the long training period involved. Also, the nonlinear part has to be separately determined to obtain a complete solution.

In a recent work [57], the higher order correlation properties of the PSK (phase shift keyed) symbols are used to identify the Volterra type nonlinear system. Interestingly, the higher order correlation functions are simplified because of the symmetric nature of these complex symbols. However, the order of the Volterra series has to be less than the number of alphabets of the transmitted symbols and a $(k+1)^{\text {th }}$ order cross correlation function is required to identify the $k^{\text {th }}$ order Volterra kernel which makes this scheme not so friendly.

As a conclusion, a good estimation of the channel parameters is necessary for a better compensation scheme. This is especially the case with fiber-wireless systems because of the involved nonlinearity, high speed connection and time variations of the wireless channel. Therefore, we shall first focus on channel estimation by transmitting a known, practically feasible training sequence.

\subsection{Fiber-Wireless Channel Estimation}

Uplink and downlink challenges are completely different in a fiber-wireless scenario. The uplink is more severely limited than the downlink because of several reasons. 
RF power fluctuation is severe in the uplink due to different user locations and path gains. Varying multipath fading conditions make this worse. In addition, the time domain dispersion occurs before nonlinear distortion and predistortion is not possible in the uplink. It is not desirable to increase the complexity of the portable unit. In light of these problems, this and subsequent chapters investigate estimation of the fiber wireless uplink and then propose an equalizer structure for the uplink. Nonlinearity of the downlink is handled by an appropriate predistortion scheme. A comprehensive discussion on both up and downlinks is given in Chapter 9.

\subsubsection{Baseband/Passband Issue}

In this preamble, we first justify a baseband approach to the passband nonlinearity. A bandpass real signal can be completely represented by its complex baseband equivalent as shown in [50]. Many other texts use the same approach. A frequency independent memoryless bandpass amplitude and phase nonlinearity can be completely described by its baseband equivalent model as shown in [46]. Following [46] many authors use a baseband model for nonlinear channel analysis. We have shown in Appendix A that, a bandpass complex nonlinearity can be represented by two orthogonal amplitude nonlinearities. Combining all these arguments the following conclusion can be made:

A bandpass nonlinear system that introduces a memoryless AM-AM and AM-PM distortions in a bandpass real signal $x(t)$, can be completely described by two baseband nonlinear amplitude distorting systems. Each of these amplitude distorting systems distort the amplitudes of the inphase and quadrature components of the baseband equivalent of the $x(t)$

Using this approach, the nonlinear distortion is analyzed at baseband by treating the inphase and quadrature channels separately. Several previous works including [46] and [58] have used the same approach. 


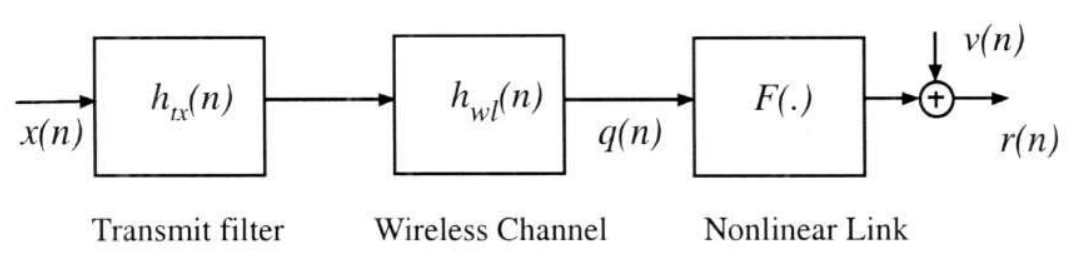

Figure 6.3: Block diagram of the fiber-wireless Uplink

\subsubsection{PN Sequence Correlation Method}

Input/output higher order correlation functions with Gaussian inputs are widely used for identification of Wiener/Hammerstein type systems [59], [55]. However, due to the practical difficulties in generating Gaussian inputs, different approaches have been proposed.

Pseudorandom (PN) sequences have white noise like properties, with respect to spectrum and the probability density functions. They are easy to generate and analyze and, their correlation properties are well understood [29]. Therefore, using PN sequence for channel estimation would ease the implementation. Besides, maximal length PN sequences are widely used in spread spectrum communication systems and likely to remain that way for a longtime. This approach is first investigated by Billings and Fakhouri for control system identification [30]. They have used the summation of two PN sequences to individually identify each block of a (inherently baseband) Wiener/Hammerstein system in the continuous time domain.

This approach is appropriately modified here to suit the (passband) communication environment. We use a discrete time domain to ease DSP implementations. Instead of using higher order correlation functions to directly estimate higher order Volterra kernels, which lead to anomalies, we use a more efficient orthogonal transform method to separate the first order correlation function using a Vandermonde matrix. This enables a fast estimation of the linear portion, independent of the complexity of the nonlinear portion. Using a suitable algorithm, such as the least squares algorithm or the triangular decomposition algorithm, a fast estimation of the nonlinear portion can be performed. 


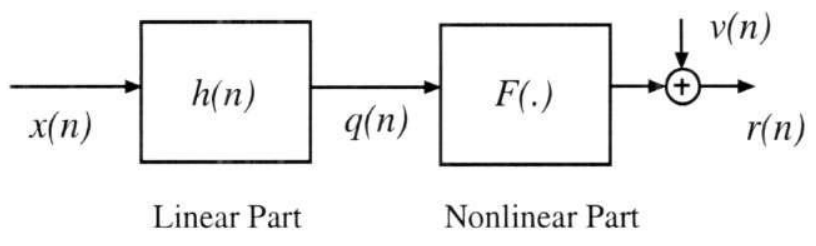

Figure 6.4: Reduced block diagram of the fiber-wireless uplink

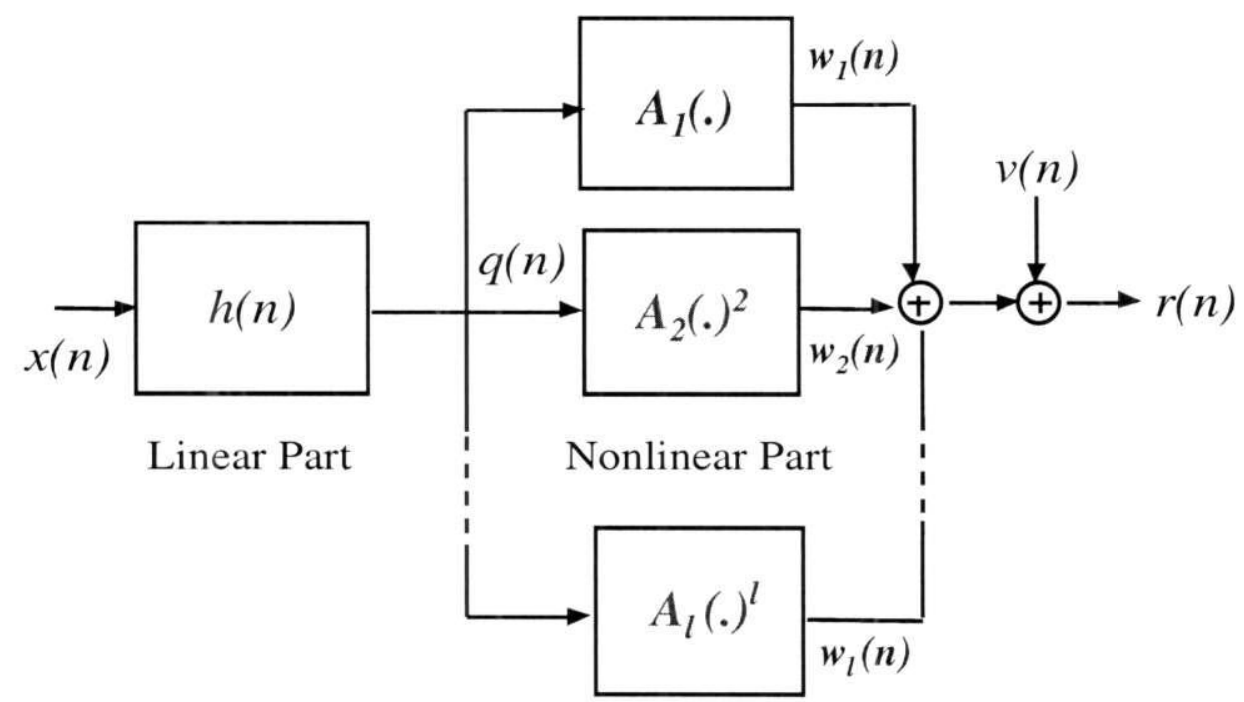

Figure 6.5: Block diagram of the fiber-wireless uplink showing separable Volterra kernels

\subsection{Mathematics of Channel Estimation}

A model of the fiber-wireless uplink is shown in Fig. 6.3. The transmit filter, with an impulse response $h_{t x}(n)$, is located in the portable unit. The wireless channel is an indoor multipath channel that is modeled by a tapped delay line filter. It has an impulse response $h_{w l}(n)$. The nonlinear link function $F($.$) models the complete$ optical link from the laser diode to the optical receiver including the RF amplifier.

Let us define a linear impulse response $h(n)$ as,

$$
h(n)=h_{t x}(n) * h_{w l}(n)
$$


Then, the internal signal $q(n)$ is given as,

$$
q(n)=x(n) * h(n)
$$

The received signal $r(n)$ is a nonlinear transformation of $q(n)$,

$$
r(n)=F[q(n)]+v(n)
$$

Assume that $F($.$) is continuous within the given dynamic range. Then from$ Weierstrass theorem, $F($.$) is uniformly approximated by a polynomial of order l$ with an arbitrary precision $\epsilon>0$. Thus,

$$
r(n)=A_{1} q(n)+A_{2} q^{2}(n)+\ldots \ldots A_{l} q^{l}(n)+v(n)
$$

where, $v(n)$ is the summation of all the optical and wireless channel noise. For convenience, $r(n)$ is rewritten as a sum of individual higher order terms $w_{i}(n)$. This model is shown in Fig. 6.5.

$$
r(n)=w_{1}(n)+w_{2}(n)+w_{3}(n)+\ldots w_{l}(n)+v(n)
$$

where,

$$
\begin{gathered}
w_{1}(n)=A_{1} \sum_{m=-\infty}^{\infty} h(m) x(n-m) \\
w_{2}(n)=A_{2} \sum_{m_{1}=-\infty}^{\infty} h\left(m_{1}\right) x\left(n-m_{1}\right) \sum_{m_{2}=-\infty}^{\infty} h\left(m_{2}\right) x\left(n-m_{2}\right) \\
w_{3}(n)=A_{3} \sum_{m_{1}=-\infty}^{\infty} h\left(m_{1}\right) x\left(n-m_{1}\right) \sum_{m_{2}=-\infty}^{\infty} h\left(m_{2}\right) x\left(n-m_{2}\right) \\
\sum_{m_{3}=-\infty}^{\infty} h\left(m_{3}\right) x\left(n-m_{3}\right)
\end{gathered}
$$

Finally,

$$
w_{l}(n)=A_{l} \sum_{m_{1}=-\infty}^{\infty} \ldots \sum_{m_{l}=-\infty}^{\infty} \prod_{i=1}^{l} h\left(m_{i}\right) x\left(n-m_{i}\right)
$$


This form facilitates separation of the corresponding Volterra kernels [59]. Let us define the $i^{\text {th }}$ order separable Volterra kernel $g_{i}$ as,

$$
g_{i}\left(m_{1}, m_{2}, \ldots, m_{i}\right)=A_{i} \sum_{\sigma=-\infty}^{\infty} \prod_{j=1}^{i} h\left(m_{j}-\sigma\right)
$$

This general definition includes the memory term $\sigma$. However, in our model we assume no memory for $F($.$) . So g_{i}$ reduces to,

$$
g_{i}\left(m_{1}, m_{2}, \ldots, m_{i}\right)=A_{i} \prod_{j=1}^{i} h\left(m_{j}\right)
$$

Using this kernel description, the general term $w_{i}(1 \leq i \leq l)$ is written as,

$$
w_{i}(n)=\sum_{m_{1}=-\infty}^{\infty} \ldots \sum_{m_{i}=-\infty}^{\infty} g_{i}\left(m_{1}, m_{2}, \ldots, m_{i}\right) \prod_{j=1}^{i} x\left(n-m_{j}\right)
$$

From the preceding derivation it is seen that, for a given transmitted sequence $x(n)$, the received signal $r(n)$ consists of higher order terms of the present and previous samples that are multiplied with each other.

\subsubsection{Estimation of the Linear Part}

From equations (6.13) and (6.6), the received signal $r(n)$ can be written as,

$$
r(n)=\sum_{i=1}^{l} \sum_{m_{1}=-\infty}^{\infty} \ldots \sum_{m_{i}=-\infty}^{\infty} g_{i}\left(m_{1}, m_{2}, \ldots, m_{i}\right) \prod_{j=1}^{i} x\left(n-m_{j}\right)+v(n)
$$

where, $x(n)$ is a PN sequence of length $N_{c}$. As in (6.6), $r(n)$ is simply written as,

$$
r(n)=\sum_{i=1}^{l} w_{i}(n)+v(n)
$$

Define the zero mean processes $\operatorname{rr}(n)=r(n)-\overline{r(n)}$ and $x x(n)=x(n)-\overline{x(n)}$ where the over bar denotes the mean. The covariance of $r$ and $x, \Re_{r x}$ is computed as,

$$
\Re_{r x}(\sigma)=\overline{\operatorname{rr}(n) x x(n-\sigma)}
$$


The zero mean process $\operatorname{rr}(n)$ can now be written as,

$$
\begin{array}{r}
r r(n)=\sum_{i=1}^{l} \sum_{m_{1}=-\infty}^{\infty} \ldots \sum_{m_{i}=-\infty}^{\infty} g_{i}\left(m_{1}, m_{2}, \ldots, m_{i}\right) \\
{\left[\prod_{j=1}^{i} x\left(n-m_{j}\right)-\prod_{j=1}^{i} \overline{x\left(n-m_{j}\right)}\right]+v(n)}
\end{array}
$$

If we assume no correlation between the additive noise $v(n)$ and the input sequence $x(n)$, then the covariance function $\Re_{r x}(\sigma)$ is written as,

$$
\Re_{r x}(\sigma)=\frac{\sum_{i=1}^{l} \sum_{m_{1}=-\infty}^{\infty} \ldots \sum_{m_{i}=-\infty}^{\infty} g_{i}\left(m_{1}, m_{2}, \ldots, m_{i}\right) .}{\left[\prod_{j=1}^{i} x\left(n-m_{j}\right)-\prod_{j=1}^{i} \overline{x\left(n-m_{j}\right)}\right][x(n-\sigma)-\overline{x(n-\sigma)}]}
$$

Equation (6.18) above, is actually a summation of the correlation of $x x(n)$ with each $w_{i}(n)$ of $\operatorname{rr}(n)$. This is simply written as,

$$
\Re_{r x}(\sigma)=\sum_{i=1}^{l} \Re_{w_{i} x}(\sigma)
$$

We point out that, if $\Re_{r x}(\sigma)$ is evaluated directly as defined above, it leads to anomalies ([30], [55]). This is because, although $\Re_{r x}(\sigma)$ is the first order covariance, it involves higher order correlation functions of pseudorandom sequences, which are not always computable [29]. This problem is avoided by isolating the first order covariance function $\Re_{w_{1} x}(\sigma)$ from the rest by some means. $\Re_{w_{1} x}(\sigma)$ is needed because it is directly proportional to $h(n)$.

\subsubsection{Multilevel Transmission}

The easiest approach is to repeat the training a few (say $N_{t}$ ) times with different amplitudes. This approach was originally proposed by Billings [30]. This procedure replaces (6.19) that has higher order correlation functions, with a system of $N_{t}$ simultaneous equations that have first order correlation functions only. This enables fast solution for $\Re_{w_{1} x}(\sigma)$. Let us consider multilevel input signals $\alpha_{i} x(n)$ where, 
$\alpha_{i} \neq \alpha_{j}$ for all $i \neq j$. Then,

$$
\Re_{r_{\alpha_{i}} x}(\sigma)=\sum_{j=1}^{l} \alpha_{i}^{j} \Re_{w_{j} x}(\sigma) \quad i=1,2, \ldots, N_{t}
$$

Note that $N_{t}$ does not have to be the same as the order of the nonlinearity, since $N_{t}$ is not directly used to compute the polynomial coefficients. $N_{t}$ can be as low as 2 or 3 . The simulation shows excellent results with $N_{t}=3$.

Expanding equation (6.20) in matrix form gives,

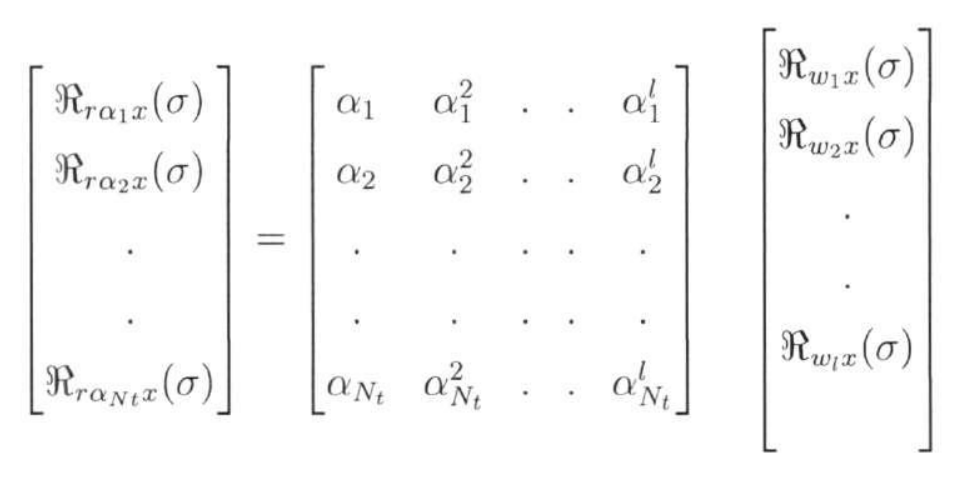

The coefficient matrix $\boldsymbol{\alpha}$ can be rewritten as,

$$
\left[\begin{array}{ccccc}
\alpha_{1} & 0 & 0 & \ldots & 0 \\
0 & \alpha_{2} & 0 & \ldots & 0 \\
0 & 0 & . & \ldots & \ldots \\
\ldots & \ldots & 0 & . & \ldots \\
0 & 0 & \ldots & 0 & \alpha_{N_{t}}
\end{array}\right]\left[\begin{array}{ccccc}
1 & \alpha_{1} & \alpha_{1}^{2} & . & \alpha_{1}^{l-1} \\
1 & \alpha_{2} & \alpha_{2}^{2} . & . & \alpha_{2}^{l-1} \\
\cdot & \cdot & \cdot & \cdot \\
\cdot & \cdot & \cdot & \cdot & \cdot \\
1 & \alpha_{N_{t}} & . \alpha_{N_{t}}^{2} & \ldots & \alpha_{N_{t}}^{l-1}
\end{array}\right]
$$

The first diagonal matrix above, is non-singular for all $\alpha_{i} \neq 0$. The second matrix is a Vandermonde matrix. The determinant of the Vandermonde matrix is given below and non zero for $\alpha_{i} \neq \alpha_{j}$

$$
\prod_{1 \leq i \leq j \leq N_{t}}\left(\alpha_{i}-\alpha_{j}\right)
$$

Thus, for every value of $\sigma$, equation (6.20) has a unique solution for $\Re_{w_{i} x}(\sigma) ; i=$ $1,2, \ldots, N_{t}$. 
Using equation $(6.7), \Re_{w_{1} x}(\sigma)$ can be shown to be given by,

$$
\Re_{w_{1} x}(\sigma)=A_{1} \sum_{m=-\infty}^{\infty} h(m) \Re_{x x}(\sigma-m)
$$

where, $R_{x x}(\sigma)$ is the auto-covariance of the PN sequence $x(n)$ and is defined as [29],

$$
\Re_{x x}(\sigma)=\sum_{m_{1}=0}^{N_{c}-1} x(n) x(n+\sigma)
$$

The function $\Re_{x x}(\sigma)$ is periodic and is easily determined if it is a maximal length sequence $^{2}$. In this case, since $x(n)$ has a unit amplitude,

$$
\Re_{x x}(\sigma)= \begin{cases}1 & \text { if } \sigma=0 \bmod N_{c} \\ -1 / N_{c} & \text { if } \sigma \neq 0 \bmod N_{c}\end{cases}
$$

Assume chip level synchronization is achieved and $N_{c}>>1$. If the correlation is computed within the time period $0 \leq n \leq N_{c}-1$, then $\Re_{x x}(\sigma)$ is given by $\Re_{x x}(\sigma)=\delta(\sigma)$. Therefore, equation (6.22) simplifies into,

$$
\Re_{w_{1} x}(\sigma)=A_{1} \sum_{m=0}^{N_{c}-1} h(m) \delta(\sigma-m)
$$

Using the convolution properties of the impulse function [50] the above reduces to,

$$
\Re_{w_{1} x}(\sigma)=A_{1} h(\sigma)
$$

which is same as,

$$
\Re_{w_{1} x}(n)=A_{1} h(n)
$$

The above is the impulse response of the unknown linear channel $h(n)$ multiplied by the constant gain $A_{1}$ of the nonlinear channel. Therefore, we have estimated the unknown linear channel impulse response. Note that the length of the PN sequence, must be larger than the channel memory for a complete identification of

\footnotetext{
${ }^{2}$ Maximal length sequences are generated from feedback binary shift registers and satisfy shift and add properties [29]
} 
$h(n)$. Resolution of the estimation depends on the chip timing.

\subsubsection{Estimation of the Nonlinear Part}

Having identified the linear part of the Wiener system, the task is to identify the nonlinear part. With the transmitted training sequence $x(n)$ known and the impulse response of the linear part $h(n)$ estimated, the unknown internal signal $q(n)=$ $x(n) * h(n)$, can be estimated. The output of the nonlinear part $r(n)$ in response to $q(n)$ is already known at the receiver. The task now is to estimate the polynomial coefficients $A_{i}(1 \leq i \leq l)$ to satisfy the relationship in 6.5. That is,

$$
r(n)=\sum_{i=1}^{l} A_{i} q^{i}(n)+v(n)
$$

This can be done in several ways. Let us review two such methods.

\subsubsection{The Least Squares Polynomial Fit}

In this curve fitting method, the goal is to fit a set of sample points to a polynomial, so that the squared error between the actual sample points and the polynomial estimate is minimized. This method requires the order of the polynomial $l$ to be smaller than the number of sample points $N_{L}$, which is a very loose condition (usually $l<<N_{L}$ ). The problem is equivalent to solving an over determined system with $N_{L}$ equations and $l$ unknowns in the least squares sense. Here, $N_{L}=N_{c} \times N_{t}$. $N_{c}$ is the length of the PN sequence and $N_{t}$ is the number of levels transmitted.

Let the estimated signal $\hat{r}(n)$ be given as a function of the estimated coefficients $\hat{A}_{i}$

$$
\hat{r}(n)=\hat{A}_{1} q(n)+\hat{A}_{2} q^{2}(n)+\ldots \ldots \hat{A}_{l} q^{l}(n)+v(n)
$$

Now, the target is to find a polynomial coefficient vector $\hat{\boldsymbol{A}}$ of length $(l+1)$ in a least squares sense such that,

$$
\hat{\boldsymbol{A}}=\arg \left[\min _{\hat{\boldsymbol{A}}} \sum_{i=1}^{N_{L}}\left[r_{i}-\hat{r}_{i}\left(q_{i} ; \hat{A}_{0} \cdots \hat{A}_{l}\right)\right]^{2}\right] .
$$


Note that, the lowest possible value for $N_{L}$ is equal to the polynomial order $l$; $N_{L}=l$. In this special case, it is referred to as an exact polynomial fit. However, in the exact fit case, this method can run into numerical problems, especially when the polynomial order is high [60].

\subsubsection{QR Decomposition Method}

Let us define vectors $\boldsymbol{q}$ and $\boldsymbol{r}$ of length $N_{L}$, that are made up of the signals $q(n)$ and $r(n)$ respectively. Also, let us define a Vandermonde matrix $\boldsymbol{V}_{\boldsymbol{q}}$ such that each row of $\boldsymbol{V}_{\boldsymbol{q}}$ is a polynomial of the corresponding data point in $\boldsymbol{q}$, so that $\boldsymbol{V}_{\boldsymbol{q}}$ has $N_{L}$ rows and $(l+1)$ columns. There is no relationship between this $\boldsymbol{V}_{\boldsymbol{q}}$ and the previous Vandermonde matrix $\boldsymbol{\alpha}$ in Section 6.5.1.1.

$$
\boldsymbol{V}_{\boldsymbol{q}}=\left[\begin{array}{ccccc}
q^{l}(1) & q^{l-1}(1) & \ldots & q(1) & 1 \\
q^{l}(2) & q^{l-1}(2) & \ldots & q(2) & 1 \\
\ldots & \ldots & \ldots & \ldots & 1 \\
\ldots & \ldots & \ldots & \ldots & \ldots \\
q^{l}\left(N_{L}\right) & q^{l-1}\left(N_{L}\right) & \ldots & q\left(N_{L}\right) & 1
\end{array}\right]
$$

Minimization of the sum of squared errors in equation (6.30) requires a solution to the system of equations,

$$
V_{q} \hat{A}=\hat{r}
$$

An efficient way of computing $\boldsymbol{p}$ is to perform an orthogonal-triangular decomposition of the Vandermonde matrix $V_{\boldsymbol{q}}$ as follows:

$$
V_{q}=Q_{q} R_{q}
$$

where $\boldsymbol{R}_{\boldsymbol{q}}$ is an upper triangular matrix and $\boldsymbol{Q}_{\boldsymbol{q}}$ is an orthonormal matrix that has the same dimension as $\boldsymbol{V}_{\boldsymbol{q}}$. With this decomposition (known as QR decomposition), (6.32) can be rewritten as:

$$
Q_{q} R_{q} \hat{A}=\hat{r}
$$


Since the unitary matrix $\boldsymbol{Q}_{q}$ has the property that $\boldsymbol{Q}_{q}^{T}=\boldsymbol{Q}_{q}^{-1}$, this simplifies to,

$$
R_{q} \hat{A}=Q_{q}^{T} \hat{r}
$$

Note that $\boldsymbol{Q}_{q}^{T} \boldsymbol{r}$ is a vector of length $(l+1)$ Thus since $\boldsymbol{R}_{q}$ is a triangular matrix, this equation can be fast solved for $\hat{A}$ by back substitution. The mean error between the actual data $r(n)$ and the computed data is given by:

$$
\epsilon=r-V_{q} \hat{A}
$$

Finally, the order of the polynomial $l$ and the number and distribution of the data points are selected to minimize the mean squared error.

\section{The Algorithm}

1. A known training symbol is selected and multiplied by a known PN sequence of length $N_{c}$. This sequence is transmitted $N_{s}$ times $^{3}$. The ratio between the symbol and chip period is an integer. The chips are transmitted with an amplitude $\alpha_{1}$.

2. The same procedure is repeated $N_{t}$ times, each time with a different amplitude $\alpha_{i}\left(1<i \leq N_{t}\right)$.

3. At the receiver the received sequence is stored and averaged by $N_{s}$. A correlation is performed with the transmitted PN sequence and the average value of the covariance function $\Re_{r_{\alpha_{i}} x}(\sigma) ;\left(0 \leq \sigma \leq N_{c}\right)$ is computed for each level $\alpha_{i} ;\left(1 \leq i \leq N_{t}\right)$.

4. $\Re_{w_{1} x}(\sigma)$ is computed using the inverse of the Vandermonde matrix and $\Re_{r_{\alpha_{i}} x}(\sigma)$. The linear part $h(n)$ is a scaled version of $\Re_{w_{1} x}(\sigma)$.

5. The nonlinear part is estimated as follows: $\boldsymbol{q}$ is computed by convoluting $h(n)$ with $x(n)$ and $\boldsymbol{V}_{\boldsymbol{q}}$ is formed from $\boldsymbol{q}$. The QR decomposition is performed to find $\boldsymbol{R}$ and $\boldsymbol{Q}$. Then, $\hat{\boldsymbol{A}}$ is determined by solving (6.35).

\footnotetext{
${ }^{3}$ Transmission is repeated $N_{s}$ times to remove the noise by averaging
} 


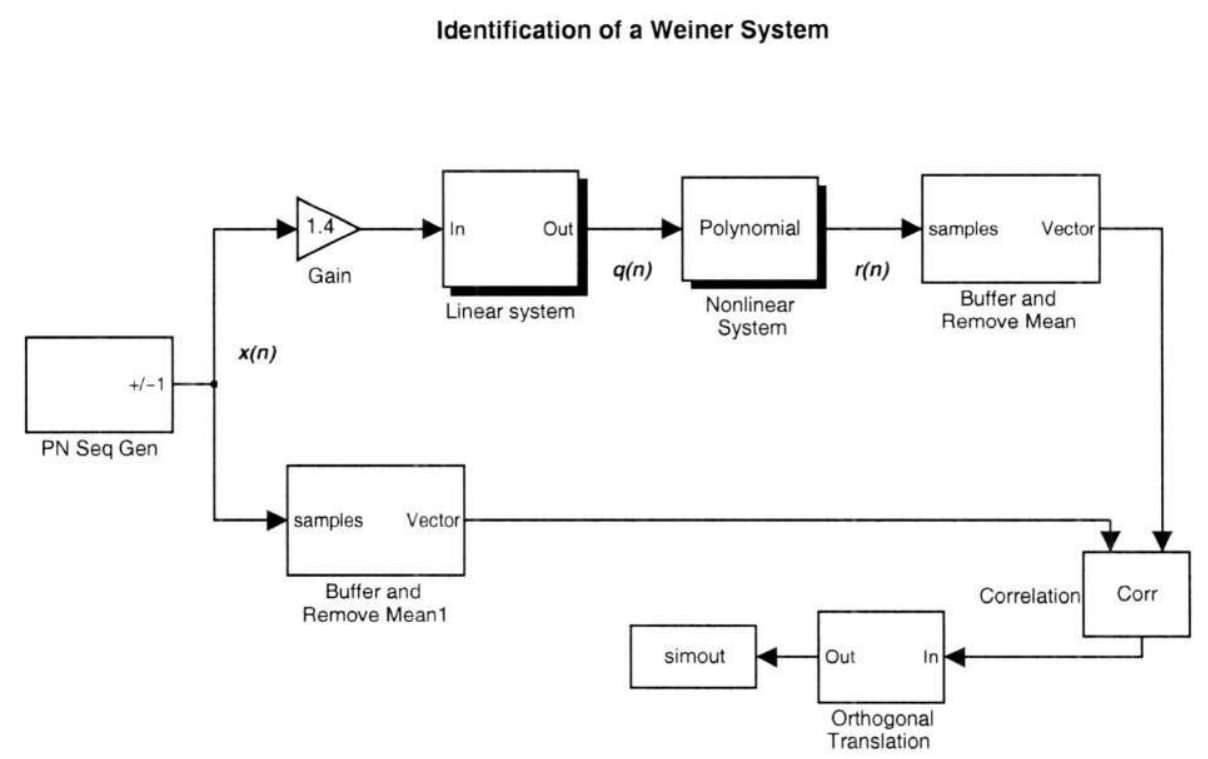

Figure 6.6: Block diagram of the simulation model in Simulink ${ }^{T M}$

\subsection{Simulation Results}

To evaluate the algorithm, the simulation is run by assuming different channel impulse responses and different nonlinear characteristics. The simulink ${ }^{T M}$ in $M A T L A B^{T M}$ environment is used for simulation.

\subsubsection{Linear System Identification}

The block diagram used for simulation is shown in Fig. 6.6. PN sequence $x(n)$ is generated from the generator block that comes with the CDMA blockset MATLAB ${ }^{T M}$ package. The gain block simulates the input level coefficient $\alpha$. The linear system is a discrete tap-delay line filter. The nonlinear system is modeled by a polynomial filter. A $30 \mathrm{~dB}$ SNR was used. The received signal $r(n)$ is buffered with a buffer of memory $N_{c}$. The correlation is done after removing the mean of the buffered $r(n)$ and $x(n)$ to yield the output $\Re_{r_{1} x}(\sigma)$. This is stored and the simulation is repeated with three different values for $\alpha\left(N_{t}=3\right)$. The values were $\alpha_{1}=1, \alpha_{2}=1.2$ and $\alpha_{3}=1.4$. Then, $\Re_{w_{i} x}(\sigma)$ is calculated using the relationship in equation (6.20)

Two different impulse responses $h(n)$ are taken. One with four paths and the 


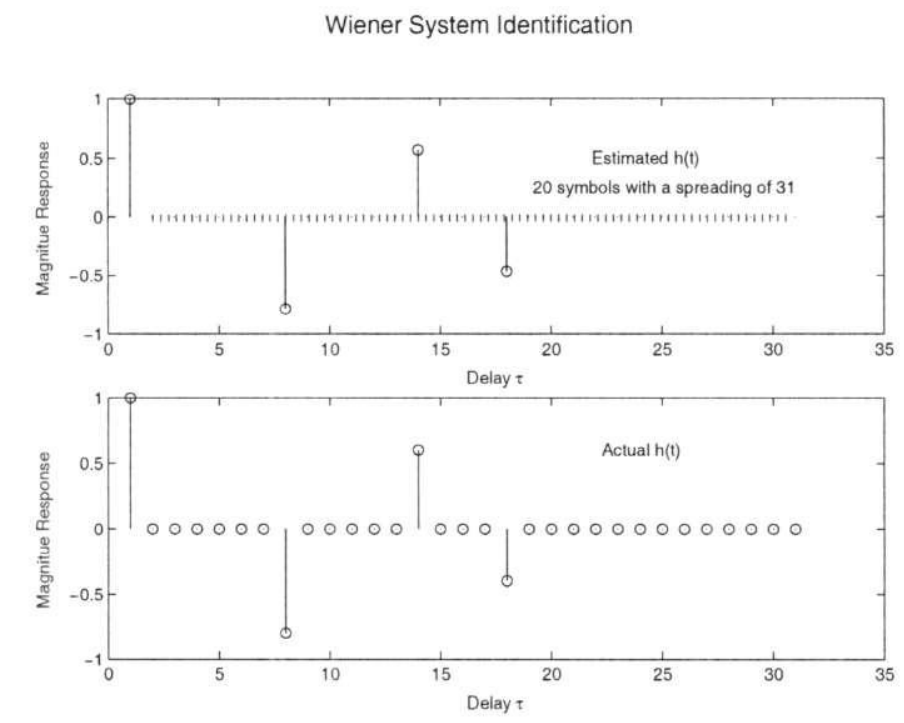

Figure 6.7: Actual and estimated wireless channel impulse responses with four discrete paths
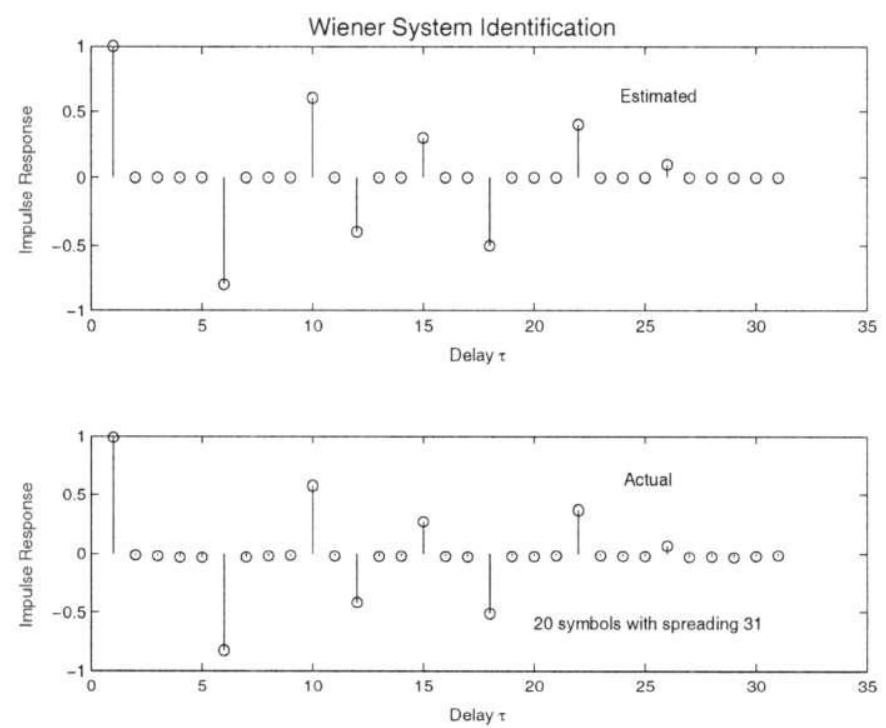

Figure 6.8: Actual and estimated wireless channel impulse responses with eight discrete paths

other one with eight paths. These are given below,

$$
h_{1}(n)=\delta(n)-0.8 \delta(n-7)+0.6 \delta(n-13)-0.4 \delta(n-17)
$$




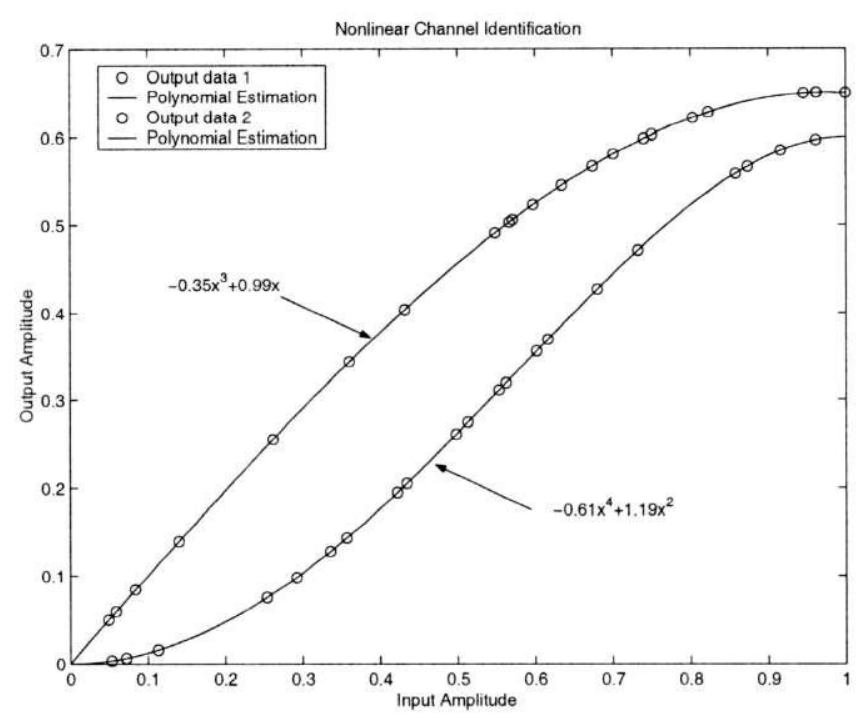

Figure 6.9: Received data and the estimated polynomial for two different cases of the nonlinear channel

$$
\begin{aligned}
h_{2}(n)= & \delta(n)-0.8 \delta(n-5)+0.6 \delta(n-9)-0.4 \delta(n-11)+0.3 \delta(n-14) \\
& -0.5 \delta(n-17)+0.4 \delta(n-21)+0.1 \delta(n-25)
\end{aligned}
$$

The original impulse response and the estimated impulse responses are shown in Fig. 6.7 and 6.8 respectively.

From the theory and simulation it is seen the suggested algorithm is very efficient in identifying Wiener type nonlinear systems. The number of levels $N_{t}$ is independent of the order of nonlinearity $l$. We used 20 symbols for training $\left(N_{s}=20\right)$ which, gives pretty good results. Depending on the noise power, fewer symbols might be enough for the estimation. Ideally (when there is no noise and there is perfect numerical precision), one symbol $\left(N_{s}=1\right)$ is enough for training. Repetition of symbols is done to remove noise by averaging. Here, the sampling rate is the same as the chip rate. Thus, the resolution of the impulse response depends on the chip time. However, the memory of the channel can be as long as the length of the PN sequence. 


\subsubsection{Nonlinear System Identification}

Identification of the linear and nonlinear systems are done independently. For the nonlinear system identification, we have considered two cases: A fourth order (even) system,

$$
r(n)=-0.6 q^{4}(n)+1.2 q^{2}(n)
$$

and a third order (odd) system,

$$
r(n)=-0.35 q^{3}(n)+q(n)
$$

The received symbols $r(n)$ and the estimated curves, using the orthogonal triangular decomposition algorithm are shown in Fig. 6.9. It is seen that the received data is closely tracked by these polynomials. The estimated polynomials from the received data using the decomposition algorithm are given by (6.41) and (6.42).

$$
\begin{aligned}
& r(n)=-0.61 q^{4}(n)+1.19 q^{2}(n) \\
& r(n)=-0.35 q^{3}(n)+0.99 q(n)
\end{aligned}
$$

The shape of the nonlinearity is immaterial for the algorithm to work. It always identifies the exact curve. The only constraint for identification is that the nonlinear function $F($.) must be single valued. Moreover, we need sample points that span the entire dynamic range of interest. This is better achieved when the linear channel has severe multipath conditions because, the received symbols will then exhibit large amplitude fluctuations. If this is not the case, then the number of levels $N_{t}$ may be increased so that, the entire dynamic range is covered. The computational complexity of this algorithm depends on the number of received sample points $N_{L}$ and the order of the polynomial $l$. 


\section{Chapter 7}

\section{Equalization of the Concatenated Fiber-Wireless Channel}

In Chapter 6, an algorithm is developed to estimate the multipath wireless channel concatenated with the nonlinear optical channel. The algorithm estimates both of these channels individually. Having known the channel, the focus of this chapter is to devise a suitable equalizer at the receiving end to cancel out the distortions introduced by the concatenated channel.

This chapter is organized as follows: First an overview is done on nonlinear equalizers. Then, nonlinear equalizers with and without a decision feedback structures are compared. This high-lights the benefits of having a decision feedback structure. Furthermore, the advantages of separating the linear and nonlinear compensation components are identified. After this, the concept Hammerstein type decision feedback equalizer (HDFE) is introduced. The HDFE consists of both linear and nonlinear filters to compensate for the linear and nonlinear channels separately. Optimization of both of these filter coefficients, with respect to the MMSE criterion, are mathematically carried out to complete the chapter.

\subsection{An Overview on Nonlinear Equalizers}

Communication channels are taken for granted as linear. This enables the usage of matured linear methods to deal with them. However, there are several occasions when the 'linearity' assumption fails, as in the case of ROF links. Nonlinear equal- 
ization is indispensable under these cases. Actually nonlinear equalizers perform better even when the channel does not explicitly have nonlinear elements. A good example is the popular decision feedback equalizer (DFE) [61].

First, let us briefly review some of the previous nonlinear equalization attempts. Lainiotis et al. [62] have categorized nonlinear equalizers into two groups depending on how the channel is estimated. Here we slightly modify that and re-group them based on how the equalizer parameters are obtained.

Explicit Equalizers The channel is explicitly identified first. That is, a parameter (and sometimes a structure) identification is performed. Then, the equalizer is configured to inverse model the channel. A training sequence is almost always required. Severe nonlinearities can be handled, since the channel is explicitly identified. However, since this is a two stage approach, the complexity is usually higher.

Implicit Equalizers Equalizer parameters are indirectly determined using some optimization criteria, such as minimizing the MSE or the BER. The attempt is to alleviate the channel distortions without an explicit channel identification. However, knowledge of the structure and the statistical properties of the channel is needed for better performance. This approach performs well when the nonlinearity is mild. Also, these equalizers have relatively low computational complexity. For these reasons, the implicit approach enjoys wide popularity.

The benefits of each of these categories can be exploited by adopting a combined approach. For example, a partial channel estimation improves the performance of an implicit equalization approach. We have also adopted a combined approach where the linear dispersive channel is estimated first (explicit approach), but the inverse of the nonlinear part is computed directly ${ }^{1}$. This approach gives good performance with a low computational overhead. Our approach is explained in detail in Section 7.1.4.

${ }^{1}$ Although in Chapter 6 , we have shown how to estimate the nonlinear part, this step can be skipped and, the inverse of the nonlinear part can be computed directly. 
Nonlinear equalizers can also be categorized based on thcir architecture. A large number of previous attempts have made use of a decision feedback architecture. This is reviewed in detail in Section 7.1.2. There are other techniques that use some different architecture but we first focus on the latter because there is relatively little work to report.

\subsubsection{Nonlinear Equalizers without a Decision Feedback}

Rolativcly, little work has been reported using nonlinear equalizers without a decision feedback structure. Probably the reason is, the requirement. for extensive processing capability and memory. This is because, in this case, a single filter attempts to inverse model both the nonlinearity and the linear channel.

There are a number of explicit equalization methods that use the Kalman filter algorithm. Some improved versions of the Kalman algorithm have also been proposed. For example, a partitioned adaptive nonlinear channel equalizer is proposed in [62] where, each transmitted symbol is treated as a state of the channel and the next state is predicted. This is similar to performing a parameter identification first. According to the authors of [62], their algorithm performs better than the classical Kalman filtcr algorithm with a comparable complexity.

Several other techniques use neural network type approaches. A multi-layer perceptron approach is proposed by Chen et al. [63] where, the equalization problem is viewed as a classification problem. The basic idea of this work is to generate a nonlinear decision region instead of a conventional linear decision region. A three layer perceptron shows good performance in this case. However, the author himself admits that, slow convergence and local minima issues have to be resolved before proposing this technique for real time implementation.

An adaptive lattice channel equalizer is analyzed in [64]. Here, a second order Volterra series is generated from the received signal (the author calls it 'multichannel'), which is used as the input to a lattice predictor. Finally, some work has been done with maximum likelihood nonlinear channel estimation [65]. However, the complexity of all of these equalizers, at present, is too high for practical realization. This is especially true with the maximum likelihood approach. 


\subsubsection{The Basic Decision Feedback Equalizer}

The decision Feedback equalizer (DFE) has been very successful in wireless communications, thanks to its robustness in mitigating commonly encountered spectral nulls in frequency selective fading channels. Therefore, a significant performance improvement is expected by enhancing the basic DFE to handle additional nonlinear distortion. Consequently, it is not surprising to see several attempts to equalize nonlinear channels by enhancing the basic DFE in some way.

Strictly speaking the basic DFE itself is nonlinear. The nonlinearity comes from the decision device and from the feedback loop [66]. The decision device basically removes any residual ISI and noise from the received (analog) symbols and decides which discrete symbol would have been transmitted. We can imagine that the channel acts as an D/A converter by adding analog noise and ISI to the transmitted discrete symbols. The decision device removes them by acting as an $\mathrm{A} / \mathrm{D}$ converter. In case of the feedback loop, it eases the equalization with less filter taps by making use of the previous (correct) decisions because, it cancels postcursor components. This concept is analogous to an IIR filter, which performs with greater parsimony with less parameters than an FIR filter. Conclusively, we can make following observations:

- Although the basic DFE is nonlinear, most of the time it is used in equalizing the linear channels.

- Both the feedback and the feed forward filters in the basic DFE are linear.

When the channel itself is nonlinear with AM-AM and AM-PM type distortions,

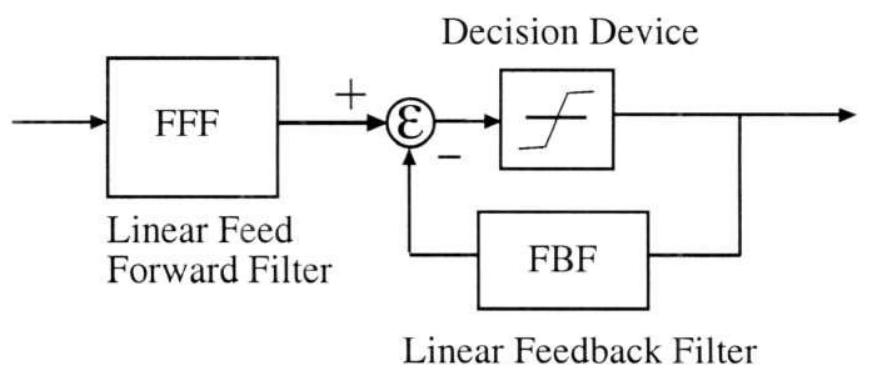

Figure 7.1: The basic decision feedback equalizer 


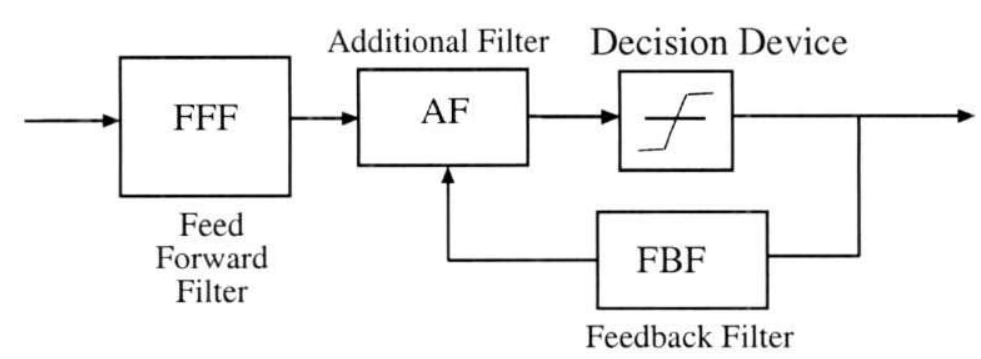

Figure 7.2: A general block diagram of some previous nonlinearly enhanced decision feedback equalizers (NEDFE)

the basic DFE is enhanced in some way to compensate the additional nonlinear distortion. We collectively refer to them as Nonlinearly Enhanced DFE (or NEDFE). Let us briefly review several NEDFE attempts now.

\subsubsection{Nonlinearly Enhanced Decision Feedback Equalizers}

Initial attempts focused on enhancing one of the linear filters of the DFE - either the feedback filter $(\mathrm{FBF})$ or the feed forward filter (FFF) - by a nonlinear filter. For example, in an earlier attempt [67], both of these filters were supplemented with Volterra type filters. Although this architecture gave reasonable performance, the complexity was too high. Namely, some 102 nonlinear tap coefficients, especially the 'cross-product coefficients' were required for satisfactory performance. Since then, several attempts have been done to conquer the complexity. A less complex version of the Volterra filter is proposed in [68], that uses only tapped delay lines and multipliers. LMS lattice linear predictors are used as the FFF and FBF in [69]. It is surprising to know that these linear predictors performed nonlinear equalization as stated in [69]. In [70], the feedback filter is replaced by the combination of a linear memory filter in series with a nonlinear memoryless filter. Actually this combination is a Wiener type filter though the author did not observe that. However, the embodiment of this filter within the DFE structure, prohibits its full benefit. This drawback, not efficiently separating the linear and nonlinear parts, increases the complexity in this category of attempts.

The second category of approaches use a slightly different architecture of the DFE. A block diagram of this architecture is shown in Fig. 7.2. An additional filter 
(AF) shown in Fig. 7.2, is used to incorporate the nonlinearity. The AF is a fuzzy logic type filter in [71]. It is a multi-layer perceptron type neural network filter in [72] and [73]. The AF is a 'generalized cerebellar model arithmetic computer' in [74] and [75]. The AF is a bilinear filter with no FFF and FBF in [76]. The FFF and FBF are linear in all other cases. In general, the additional filter significantly increases the overall complexity. For example there are hidden layers when it is a

neural network filter. In case of the bilinear filter, the equalization is inherently limited to the second order. Still the performance is better than a Volterra DFE, because of the multiplication with the feedback term.

\subsubsection{Proposal of a Hammerstein Type DFE (HDFE)}

An equalizer effectively inverse models a channel. it is desired that, the structure of the equalizer be an exact inverse of the channel structure. The inclusion of the nonlinearity in the equalizer should, therefore, be carefully planned to meet this objective while focusing on maintaining a low complexity. We show, in this dissertation, that this approach brings significant benefits.

The concatenated optical wireless uplink consists of a linear dynamic system (the wireless channel) followed by a static nonlinear system (the optical channel) with noise. It is modeled as a Wiener system. The inverse of a Wiener system is a Hammerstein system since, the nonlinearity should be compensated for first [55]. In developing the compensation scheme, we consider the following properties of the concatenated fiber-wireless channel:

1. The wireless channel is a relatively fast time varying channel. Thus, the

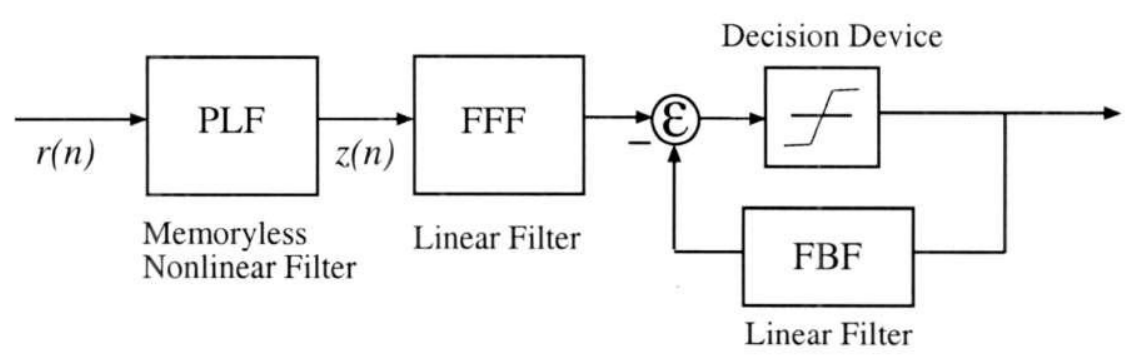

Figure 7.3: Proposed Hammerstein type decision feedback equalizer 
channel has to be frequently estimated and compensated for. Furthermore, this should be done in real time (with a short training overhead).

2. The nonlinearity comes from the cascade of the laser diode and the RF amplifier. Therefore it changes much slowly or, it is stationary compared to the wireless channel. This means that the nonlinear compensation need to be updated only occasionally compared to the frequency of the wireless channel compensation.

3. In a multiuser environment, each user is in a different location therefore, has a different wireless channel. However, they all share the same ROF link. Hence, in a multiuser environment it is possible to use a single nonlinearity compensation if it is separated from the rest of the receiver.

From the foregoing, it is obvious that an intelligent compensation scheme should separate linear and nonlinear distortion compensation. The linear (distortion compensation) part, should be updated in a relatively fast manner while, the nonlinear (distortion compensation) part can be updated less frequently. Conclusively, we propose a Hammerstein type equalizer which achieves all these observations and, therefore, highly desirable for the fiber-wireless uplink. The architecture of the HDFE is shown in Fig. 7.3 and the complete uplink with HDFE is shown in Fig. 7.4.

In these figures, the PLF is a memoryless polynomial filter of order $N$. The FFF is the linear feed forward filter and FBF is the linear feedback filter. Both of these have memory and the combination FFF and FBF with the decision device makes up the corresponding dynamic component of the Hammerstein system. As we have outlined in Section 7.1.2, this dynamic component (which is a basic DFE) is not strictly linear. However, it is well known [66] that basic DFEs well equalizes the wireless channel dispersion. On the other hand, we have an explicitly nonlinear PLF, that compensates for nonlinear distortions. This is our proposed receiver architecture for the fiber-wireless uplink where, the PLF inverse models the optical link nonlinearity while the rest equalizes the wireless channel dispersion.

The HDFE consists of both linear and nonlinear filters. The polynomial filter has zero memory, hence, it occupies a single point, or a scalar, in the time axis. 


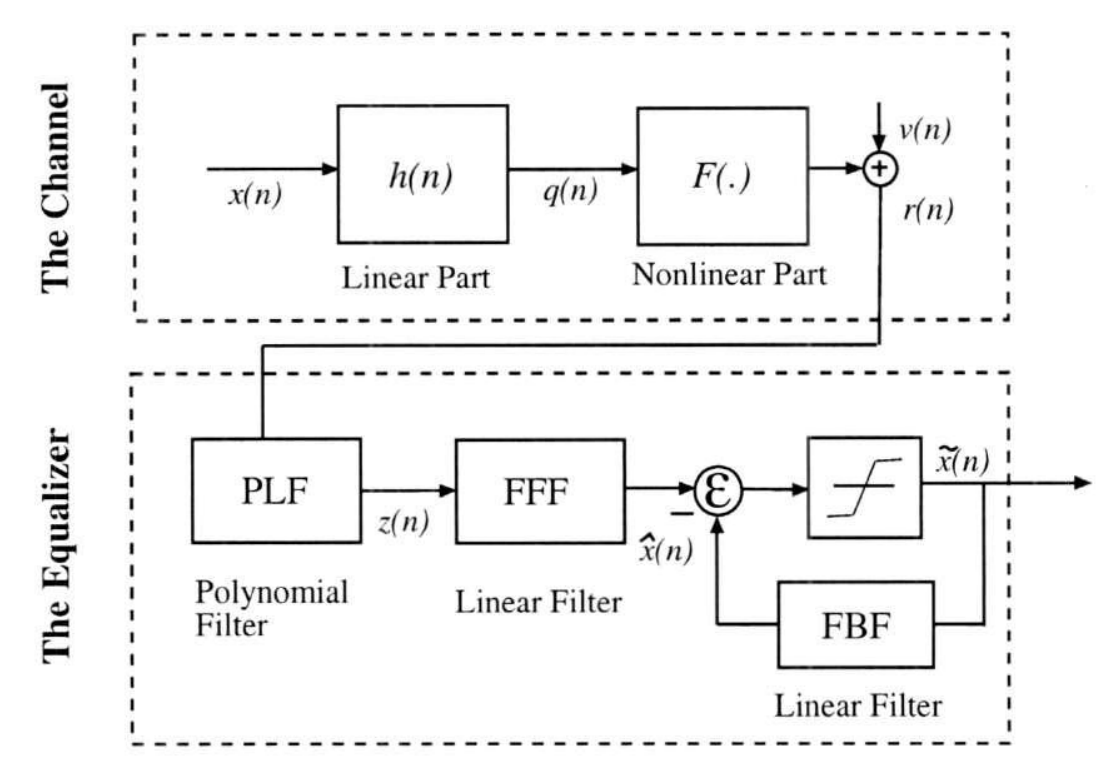

Figure 7.4: Complete block diagram of the fiber-wireless channel and the receiver

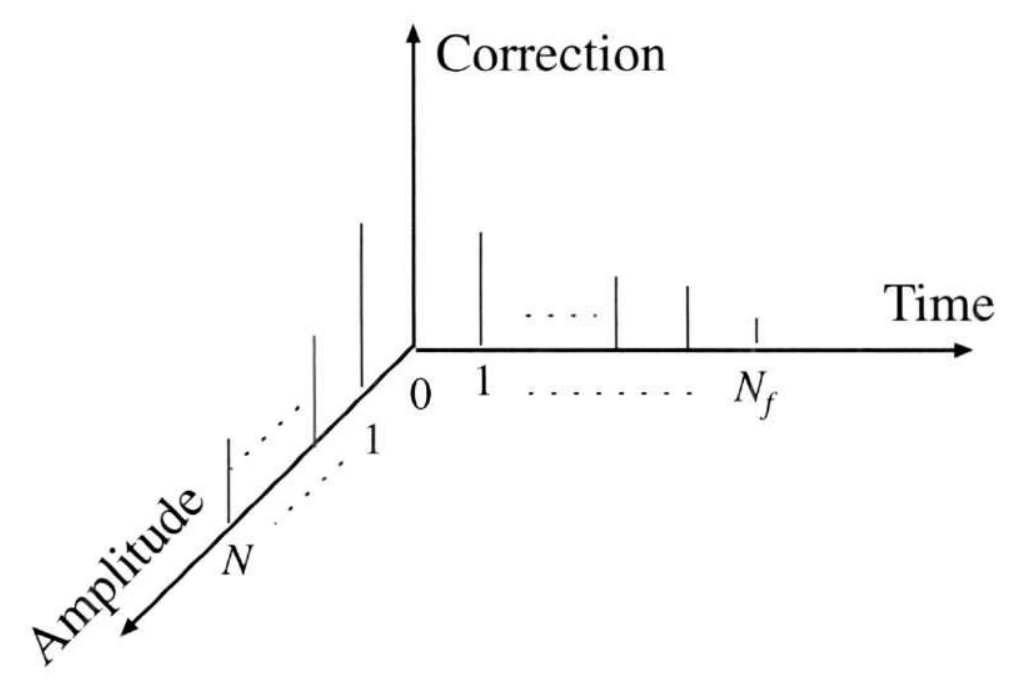

Figure 7.5: The polynomial filter and linear filters operate in two different domains

Yet, it has a vectorial expansion in the amplitude (or power) domain. On the other hand, linear filters have memory, therefore, they are vectors in the time domain. Yet they assume a single amplitude, therefore, scalars in the amplitude domain As a result, these two parts operate in two different domains, namely amplitude and time domains. Furthermore, amplitude and time domains are independent, therefore, are orthogonal to each other. This phenomenon is pictorially described 
in Fig. 7.5. From this, we observe that optimization of these two parts, linear and nonlinear filter parts, also should be done separately. Conclusively, in Section 7.2, we focus on optimizing the polynomial filter coefficients and, in the Section 7.3 we focus on optimizing the linear filter coefficients.

\subsection{Optimization of Polynomial Filter Parame- ters}

Optimization of the PLF can be done in two different ways: after explicit estimation of the channel or directly (without an explicit estimation of the channel). In the first case, we assume knowledge of channel polynomial and in the second case we do not. Both of these methods are described in sequence here.

Referring to Fig. 7.3, the output of the polynomial filter $z(n)$ has the form,

$$
z(n)=g_{0}+g_{1} r(n)+g_{2} r^{2}(n)+\ldots g_{N} r^{N}(n)
$$

where $N$ is the order of the filter. There are no delay terms since we assume no memory for the filter. The weights $g_{i}(0 \leq i \leq N)$ are determined such that, this polynomial is an inverse of the channel nonlinearity $F($.$) , at least within the given$ dynamic range. In other words, $z(n)$, which is a function of $r(n)$, should be made proportional to $q(n)$. That is,

$$
z[r(n)] \propto q(n)
$$

The weights $g_{i}$ can be determined directly or indirectly to meet this requirement. Remember that this is effectively a post-compensation scheme and we assume that there exists an inverse polynomial to satisfy this condition. We point out that, this is a very reasonable assumption and, this requirement is easily met for most practical $F($.). Also, there is an inter-relationship between the order of the compensating polynomial $(N)$, dynamic range of the input, the input noise power and the accuracy of inverse modeling which will be reflected in the MMSE. 


\subsubsection{PLF Parameters using the Channel Polynomial}

Here, we assume that we know the polynomial that represent the channel nonlinearity. Estimation procedure of the channel polynomial is described in Section 6.5.2. Using this knowledge the weights $g_{i}$ of the PLF can be generated.

Let us rewrite the nonlinear channel output as,

$$
r(n)=A_{1} q(n)+A_{2} q^{2}(n) \ldots+A_{l} q^{l}(n)+v(n)
$$

where, $A_{i}(0 \leq i \leq l)$ are the previously estimated coefficients and $v(n)$ is an unknown noise term. $q(n)$ is the input to the nonlinear channel and $r(n)$ is the output.

\subsubsection{Least Squares Fit}

In this curve fitting technique, the purpose is to find optimum weights $g_{i}$ using a set of $N_{K}$ sample points, so that the squared error between $q(n)$ and $z(n)$ is minimized. With this method, there is no restriction on the order of the polynomial $N$. When $N_{K} \geq N$, the problem is equivalent to solving an over determined system with $N_{K}$ equations and $N$ unknowns in the least squares sense.

Let us define the weight vector $\boldsymbol{G}(n)$ as,

$$
\boldsymbol{G}(n)=\left[\begin{array}{lllll}
g_{0}(n) & g_{1}(n) & g_{2}(n) & \ldots & g_{N}(n)
\end{array}\right]^{T}
$$

The optimum coefficients of the polynomial are determined by solving the following equation:

$$
\boldsymbol{G}=\arg \left[\min _{\boldsymbol{G}} \sum_{i=1}^{N_{K}}\left[q_{i}-z_{i}\left(q_{i} ; g_{0} \cdots g_{N} ; A_{1} \cdots A_{l}\right)\right]^{2}\right] .
$$

Note that in equation (7.5), $r_{i}$ does not appear explicitly. That information is embedded in $A_{i}$. Furthermore, an additional sample sequence of length $N_{K}$, which is different from the original sample sequence of length $N_{L}$, is used to determine the inverse polynomial. This sample sequence is generated in the receiver itself, and can be adjusted to span the given dynamic range evenly or unevenly as required. Also, $N_{K}$ can be smaller than $N_{L}$, which simplifies the problem. $N_{K}$ can even be 
made equal to the polynomial order $N$. In this special case, it is referred to as an exact polynomial fit. In the exact fit case, the matrix $\boldsymbol{V}_{\boldsymbol{r}}$ can be ill-conditioned, depending on the numerical values of $r(n)$. Therefore, this method can run into numerical problems, especially when the polynomial order is high [60].

In this approach, where the channel polynomial is explicitly estimated first and then the equalizer polynomial is determined, the computational complexity is too high. First, the direct polynomial is estimated which involves some matrix inversion. Then, the inverse polynomial is selected. As a result, we will not consider this approach further. This method may be attractive in situations where the direct polynomial coefficients are readily available.

\subsubsection{Direct Generation of the Inverse Polynomial}

In this section we look at the direct generation of the inverse polynomial from the received signal $r(n)$ without, an explicit estimation of the channel polynomial. This is possible because, the linear channel impulse response $h(n)$ has already been estimated and using the $h(n), q(n)$ can be estimated. Using these, the output $z[r(n)]$ can be made proportional to the $q(n)$, without explicitly knowing $A_{i}$. This can be done by either a QR decomposition method or by using adaptive algorithms.

\subsubsection{QR Decomposition Method}

For the QR decomposition method, $r(n)$ is first expanded into a Vandermonde matrix $\boldsymbol{V}_{\boldsymbol{r}}$ of $N_{L}$ rows and $(N+1)$ columns. Recall that $N_{L}$ is the number of sample points in $r(n)$.

$$
\boldsymbol{V}_{\boldsymbol{r}}=\left[\begin{array}{cccccc}
1 & r(1) & r^{2}(1) & \ldots & r^{N-1}(1) & r^{N}(1) \\
1 & r(2) & r^{2}(2) & \ldots & r^{N-1}(2) & r^{N}(2) \\
1 & \ldots & \ldots & \ldots & \ldots & \ldots \\
\ldots & \ldots & \ldots & \ldots & \ldots & \ldots \\
1 & r\left(N_{L}\right) & r^{2}\left(N_{L}\right) & \ldots & r^{N-1}\left(N_{L}\right) & r^{N}\left(N_{L}\right)
\end{array}\right]
$$


The expression in (7.1)can be rewritten in matrix form as,

$$
V_{r} G=z
$$

Define the vector $\boldsymbol{q}$ of length $N_{L}$ as,

$$
\boldsymbol{q}=\left[\begin{array}{lllll}
q(1) & q(2) & q(3) & \ldots & q\left(N_{L}\right)
\end{array}\right]^{T}
$$

Note that the elements in $\boldsymbol{q}$ are obtained by convolving the transmitted signal $x(n)$ and the estimated channel impulse response $h(n)$. Now, the object is to determine the weights of the vector $\boldsymbol{G}$ such that,

$$
V_{r} G=\hat{q}
$$

where, $\hat{\boldsymbol{q}}$ is an estimate of $\boldsymbol{q}$. This $\hat{\boldsymbol{q}}$ should closely resemble $\boldsymbol{z}$. An efficient way of computing $\boldsymbol{G}$ is to perform an orthogonal-triangular decomposition of the Vandermonde matrix $\boldsymbol{V}_{\boldsymbol{r}}$, that is,

$$
V_{r}=Q_{r} R_{r}
$$

where, $\boldsymbol{R}_{\boldsymbol{r}}$ is an upper triangular matrix and $\boldsymbol{Q}_{\boldsymbol{r}}$ is an orthonormal (unitary) square matrix that has the same rank as $\boldsymbol{V}_{\boldsymbol{r}}$. Equation (7.9) can now be written as,

$$
Q_{r} R_{r} G=\hat{q}
$$

Since the unitary matrix $Q_{r}$ has the property of $Q_{r}^{T}=Q_{r}^{-1},(7.11)$ simplifies to,

$$
R_{r} G=Q_{r}^{T} \hat{q}
$$

Note that $Q_{r}^{T} \hat{\boldsymbol{q}}$ is a vector of length $N_{L}$. Since $\boldsymbol{R}_{r}$ is a triangular matrix this equation is fast solved for $\boldsymbol{G}$ by back substitution. The error between the actual data $\boldsymbol{q}$ and the computed data $\hat{\boldsymbol{q}}=\boldsymbol{z}$ is given by,

$$
\epsilon_{p}=q-\hat{q}=q-z
$$

The filter order $N$ is increased iteratively until this error is adequately minimized. 
This method is very attractive compared to the explicit method with respect to the number of computational steps involved.

\subsubsection{Adaptive Methods}

In this section we look at obtaining the weight vector $\boldsymbol{G}$ by adaptive means. The objective of an adaptive algorithm is to update the weights $\boldsymbol{G}$ so that, the estimation error $\boldsymbol{\epsilon}_{\boldsymbol{p}}(\boldsymbol{n})$ between the desired output $\boldsymbol{q}$ and the PLF output $\boldsymbol{z}$ is minimized in a mean-squared sense.

The recursive least squares (RLS) algorithm is preferred due to its fast convergence properties with correlated input. Adjacent samples of $r(n)$ are correlated in this application since, the input amplitude changes slower than the sampling rate. According to the RLS algorithms, the exponentially weighted cost function in (7.14) is minimized, where $\lambda_{f f}$ is the forgetting factor $\left(0.95 \leq \lambda_{f f} \leq 1\right)$ that determines the weight of previous samples, in a non-stationary environment. Throughout this dissertation we assumed a stationary environment and took $\lambda_{f f}=1$

$$
J(n)=\sum_{k=1}^{n} \lambda_{f f}^{n-k}\left[q_{k}-z_{k}\left(r_{k} ; g_{o}, \cdots, g_{N}\right)\right]^{2}
$$

Let us define the input vector $\boldsymbol{U}_{r}(n)$ of the PLF as,

$$
\boldsymbol{U}_{r}(n)=\left[\begin{array}{lllll}
1 & r(n) & r^{2}(n) & \ldots & r^{N}(n)
\end{array}\right]^{T}
$$

So that, the output $z(n)$ is given by,

$$
z(n)=\boldsymbol{U}_{r}^{T}(n) \boldsymbol{G}(n)
$$

Note that $z(n)$ is a scalar quantity because of the inner product.

According to [47], the optimal solution for $\boldsymbol{G}$, which minimizes $J(n)$, is given by,

$$
\boldsymbol{G}^{o p}(n)=\Re_{u u}^{-1}(n) \Re_{u q}(n)
$$

Here, $\Re_{u u}(n)$ is the auto-correlation matrix of the input vector $\boldsymbol{U}_{\boldsymbol{r}}(n)$ as defined in 
(7.18).

$$
\boldsymbol{\Re}_{u u}(n)=\sum_{k=1}^{n} \lambda_{f f}^{n-k}\left[\boldsymbol{U}_{r}(k) \boldsymbol{U}_{r}^{T}(k)\right]
$$

$\Re_{u q}(n)$ is the cross correlation vector between the input vector $\boldsymbol{U}_{\boldsymbol{r}}(n)$ and the desired response $\boldsymbol{q}$ as defined in (7.19). The RLS algorithm implements (7.17) recursively $[47]$.

$$
\Re_{u q}(n)=\sum_{k=1}^{n} \lambda_{f f}^{n-k}\left[\boldsymbol{U}_{r}(\boldsymbol{k}) \boldsymbol{q}(\boldsymbol{k})\right]
$$

After adequate training, if the MMSE is still high then the training is repeated with a different filter order $N$. Within a given dynamic range, in general, the accuracy of the polynomial filter compensation improves with the number of available samples and the order of the compensating polynomial. This increment, however, is not linear.

\subsection{Optimization of Linear Filter Parameters}

In this section, tap coefficients are derived for the FFF and FBF. Assuming that the channel nonlinearity is completely compensated by the PLF, these linear filter coefficients are optimized to equalize for the wireless channel dispersion only.

The equalizer coefficients can be optimized using different criteria such as the peak distortion criterion or the MMSE criterion [66]. Since the MMSE criterion is more prevalent in practice, we focus on it. Under the MMSE criterion, two different approaches for computing the tap coefficients exist, i.e., simultaneous optimization and separate optimization of FFF and FBF. In simultaneous optimization, the FFF and FBF coefficients are calculated jointly under the MMSE criterion. This is done, either by seeking the global minimum of the quadratic error surface, or by using the orthogonality principle. These two approaches are mathematically equivalent [47] The filter coefficients are then calculated by inverting the resulting autocorrelation matrix. Proakis [50] has used the joint approach. In case of the separate MMSE optimization, the postcursor ISI and the precursor ISI are treated individually [77].

As shown by Beaulieu and Smee in [78], the simultaneous and separate optimization approaches yield identical results when the noise is not correlated with the signal and white. Nevertheless, the separate optimization approach gives more 


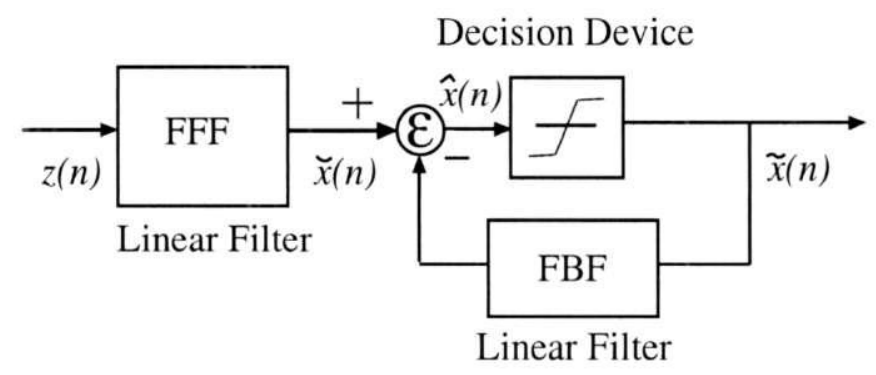

Figure 7.6: Decision feedback part of the proposed HDFE receiver

freedom in handling each filter individually. This is especially convenient in investigating the finite length effects of the filters [79].

\subsubsection{Model Description}

The DFE architecture depicted in Fig. 7.6 consists of two filters, an anti-causal feed forward filter (FFF) with $N_{f}$ taps and a causal feedback filter (FBF) with $N_{b}$ taps; both have taps spaced at the symbol (which is a chip here) interval $T_{c}$. Referring to Fig. 7.4 on page 133, the input $z(n)$ to the feed forward filter is the output of the PLF. Furthermore, $q(n)$ is the linear channel output and $z(n)$ closely resembles $q(n)$, except for the small error as shown in (7.13) on page 137. Therefore, $z(n)$ is written as,

$$
z(n)=\sum_{m=0}^{L} h_{m} x(n-m)+n_{p}(n)
$$

where, $L$ is the channel memory and

$$
n_{p}(n)=\eta(n)+\epsilon_{p}(n)
$$

Here, $\eta$ is the noise at the input of the PLF and $\epsilon_{p}$ is the error added at the PLF. Define the following vectors: a block of the transmitted data, with a block size of $N_{f}+L$ as,

$$
\boldsymbol{x}(n)=\left[\begin{array}{llll}
x\left(n+N_{f}-1\right) & x\left(n+N_{f}-2\right) & \cdots & x(n-L)
\end{array}\right]^{T}
$$


a block of $z(n)$ of length $N_{f}$ as,

$$
\boldsymbol{z}(n)=\left[\begin{array}{llll}
z\left(n+N_{f}-1\right) & z\left(n+N_{f}-2\right) & \ldots & z(n)
\end{array}\right]^{T}
$$

and a block of noise samples of length $N_{f}$ as,

$$
\boldsymbol{n}_{p}(n)=\left[\begin{array}{llll}
n_{p}\left(n+N_{f}-1\right) & n_{p}\left(n+N_{f}-2\right) & \ldots & n_{p}(n)
\end{array}\right]^{T}
$$

Then, (7.20) can be rewritten in matrix notation as,

$$
\boldsymbol{z}(n)=\boldsymbol{H} \boldsymbol{x}(n)+\boldsymbol{n}_{p}(n)
$$

where, $\boldsymbol{H}$ is the channel impulse response matrix of dimension $\left(N_{f} \times\left(N_{f}+L\right)\right)$,

$$
\boldsymbol{H}=\left[\begin{array}{ccccccc}
h_{0} & h_{1} & \ldots & h_{L} & 0 & \ldots & 0 \\
0 & h_{0} & h_{1} & \ldots & h_{L} & 0 & \ldots \\
\vdots & & & & & & \vdots \\
0 & \ldots & 0 & h_{0} & h_{1} & \ldots & h_{L}
\end{array}\right]
$$

Next, let us define the data autocorrelation matrix $\Re_{x x}$, that is a square Hermitian matrix of dimension $\left(N_{f}+L\right)$ as,

$$
\Re_{x x}=E\left[\boldsymbol{x}(n) \boldsymbol{x}^{H}(n)\right]
$$

and the $N_{f}$ dimensional noise correlation matrix as,

$$
\Re_{n n}=E\left[\boldsymbol{n}_{p}(n) \boldsymbol{n}_{p}^{H}(n)\right]
$$

Note that we have not assumed any statistical properties for $\boldsymbol{x}(n)$ and $\boldsymbol{n}_{\boldsymbol{p}}(n)$ above. Define the $N_{f}$ dimensional autocorrelation matrix of $\boldsymbol{z}(n)$ as,

$$
\Re_{z z}=E\left[\boldsymbol{z}(n) \boldsymbol{z}^{H}(n)\right]
$$

By substituting for $(7.25) \boldsymbol{z}(n)$ and assuming no correlation between noise and 
signal, $\Re_{z z}$ can be written as,

$$
\Re_{z z}=\boldsymbol{H} \Re_{x x} \boldsymbol{H}^{H}+\Re_{n n}
$$

Finally, the $\left(N_{f}+L\right) \times N_{f}$ dimensional cross correlation matrix $\Re_{x z}$ is written as

$$
\begin{aligned}
\Re_{x z} & =E\left[x(n) \boldsymbol{z}^{H}(n)\right] \\
& =\Re_{x x} \boldsymbol{H}^{H}
\end{aligned}
$$

assuming no correlation between noise and signal. Note that this cross correlation is same as the channel impulse response when $\boldsymbol{x}(n)$ is white.

From Fig. 7.6 on page 140, the equalizer output is expressed as,

$$
\hat{x}(n)=\sum_{i=-N_{f}+1}^{0} w_{i} z(n-i)-\sum_{i=1}^{N_{b}} w_{i} \tilde{x}(n-i)
$$

where, $\hat{x}(n)$ is an estimate of the $n^{\text {th }}$ information symbol. $w_{i}$ are tap coefficients of the filters and $\left\{\tilde{x}(n-1) \ldots \tilde{x}\left(n-N_{b}\right)\right\}$ are previously detected symbols. The equalizer is assumed to have $N_{f}$ taps in its feed forward section and $N_{b}$ taps in its feedback section. The combined weight vector of both filters is given as,

$$
\boldsymbol{W}=\left[\begin{array}{ll}
\boldsymbol{W}_{f}^{T} & \boldsymbol{W}_{\boldsymbol{b}}^{T}
\end{array}\right]^{T}
$$

where, $\boldsymbol{W}_{\boldsymbol{f}}$ and $\boldsymbol{W}_{\boldsymbol{b}}$ are defined as,

$$
\begin{gathered}
\boldsymbol{W}_{\boldsymbol{f}}=\left[\begin{array}{llll}
w_{-N_{f}+1} & w_{-N_{f}+2} & \ldots & w_{0}
\end{array}\right]^{T} \\
\boldsymbol{W}_{\boldsymbol{b}}=\left[\begin{array}{lll}
w_{1} & \ldots & w_{N_{b}}
\end{array}\right]^{T}
\end{gathered}
$$

\subsubsection{Parameter Optimization}

Due to the non-ideal FFF and FBF tap weights there is always an error between $x(n)$ and $\hat{x}(n)$. In this section, the filter weights are optimized to minimize the 
mean squared value of this error. The linear filter error sequence $\epsilon_{l}$ is given by,

$$
\begin{aligned}
\epsilon_{l}(n) & =x(n)-\hat{x}(n) \\
& =x(n)-\sum_{i=-N_{f}+1}^{0} w_{i} z(n-i)+\sum_{i=1}^{N_{b}} w_{i} \tilde{x}(n-i)
\end{aligned}
$$

The feedback vector $\boldsymbol{W}_{\boldsymbol{b}}$ is augmented, for convenience, as below,

$$
\widetilde{\boldsymbol{W}}_{b}=\left[\begin{array}{llllll}
\mathbf{0}_{1 \times \Delta} & 1 & w_{1} & \ldots & w_{N_{b}} & \mathbf{0}_{1 \times\left(N_{f}+L-\Delta-N_{b}-1\right)}
\end{array}\right]^{T}
$$

where, $\Delta\left(0 \leq \Delta \leq N_{f}+L-1\right)$ is the delay of the feed forward filter. This delay is adjusted to minimize the mean squared error. Note that, the number of additional zeros are adjusted in way such that the length of $\widetilde{\boldsymbol{W}}_{b}$ is $N_{f}+L$. Therefore, $\widetilde{\boldsymbol{W}}_{b}$ can be multiplied with $\boldsymbol{x}(\boldsymbol{n})$. This also implies that $\left(N_{f}+L\right)>\left(N_{b}+1\right)$ which is always true because usually $N_{b} \approx L$. Now (7.36) is written as,

$$
\epsilon_{l}(n)=\widetilde{\boldsymbol{W}}_{b} \boldsymbol{x}(\boldsymbol{n})-\boldsymbol{W}_{f} \boldsymbol{z}(n)
$$

and the mean squared error $J_{l}$ is,

$$
\begin{aligned}
J_{l} & =E[x(n)-\hat{x}(n)]^{2} \\
& =\widetilde{\boldsymbol{W}}_{b}^{H} \Re_{x x} \widetilde{\boldsymbol{W}}_{b}-\widetilde{\boldsymbol{W}}_{b}^{H} \boldsymbol{\Re}_{x z} \boldsymbol{W}_{f}-\boldsymbol{W}_{f}^{H} \boldsymbol{\Re}_{z x} \widetilde{\boldsymbol{W}}_{b}+\boldsymbol{W}_{f}^{H} \Re_{z z} \boldsymbol{W}_{f}
\end{aligned}
$$

Under practical SNR conditions the BER is $10^{-6}$ or more. Hence, it is reasonable to assume that previously detected symbols are correct. Then, the MSE, which is a quadratic function of the error $\epsilon_{l}(n)=x(n)-\hat{x}(n)$ is minimized with respect to the tap weights. This minimization is equivalent to forcing the error $\epsilon_{l}$ to be orthogonal to the signal samples $\boldsymbol{z}[47]$. Hence,

$$
\begin{aligned}
& E\left[\boldsymbol{z}^{H} \epsilon_{l}(n)\right]=0 \\
& \Rightarrow \widetilde{\boldsymbol{W}}_{b}^{H} \Re_{x z}=\boldsymbol{W}_{f}^{H} \Re_{z z}
\end{aligned}
$$


Combining (7.39) and (7.40) and substituting for $\boldsymbol{W}_{f}^{H}$, we obtain,

$$
J_{l}=\widetilde{\boldsymbol{W}}_{b}^{H} \Re_{x / z} \widetilde{\boldsymbol{W}}_{b}
$$

where $\Re_{x / z}$ is given by,

$$
\Re_{x / z}=\Re_{x x}-\Re_{x z} \Re_{z z}^{-1} \Re_{z x}
$$

In order to find the inverse of $\boldsymbol{R}_{x / z}$, let us take a Cholesky factorization of $\boldsymbol{R}_{x / z}^{-1}$,

$$
\Re_{x / z}^{-1}=\Lambda D \Lambda^{H}
$$

where, $\boldsymbol{\Lambda}$ is an $\left(N_{f}+L\right)$-dimensional lower-triangular monic matrix and $\boldsymbol{D}$ is a $\left(N_{f}+L\right)$-dimensional diagonal matrix. $\boldsymbol{D}$ can also be written as,

$$
\boldsymbol{D}=\operatorname{diag}\left(d_{0}, d_{1}, \ldots, d_{N_{f}+L-1}\right)
$$

Using factorization, it can be shown that the mean squared error is

$$
J_{l}=M S E=\widetilde{\boldsymbol{W}}_{b}^{H}\left\{\Lambda^{H}\right\}^{-1} D^{-1} \Lambda^{-1} \widetilde{\boldsymbol{W}}_{b}
$$

In order to cancel all the post-cursor interference, the number of feedback taps $N_{b}$ should be larger than the channel memory $\left(N_{b} \geq L\right)$ [50]. Probably the most parsimonious solution is taking $N_{b}=L$ (assuming we know $L$ ). In this case, Cioffi et al. [79] have shown that the MSE is minimized by choosing $\Delta$ such that $\widetilde{\boldsymbol{W}}_{b}$ matches the column of $\Lambda$ whose index is equal to the maximum entry in the diagonal matrix $\boldsymbol{D}$. That is,

$$
\max \left\{d_{N_{f}-1}, d_{N_{f}}, \ldots, d_{N_{f}+L-1}\right\}
$$

This index is equal to the optimum delay $\Delta_{o p t}$. This can be mathematically written as in (7.46). Finally, $\boldsymbol{W}_{f}$ is found by substituting for $\widetilde{\boldsymbol{W}}_{b}$ in (7.40).

$$
\widetilde{\boldsymbol{W}}_{b}^{\text {opt }}=\Lambda\left(\text { all rows },\left(\Delta_{\text {opt }}+1\right)^{t h} \text { column }\right)
$$




\subsection{Concluding Remarks}

In this Chapter, we have proposed a Hammerstein type decision feedback equalizer for compensating Wiener type nonlinear channels. We adapt the following method to optimize filter parameters. The polynomial filter parameters are directly optimized by performing QR decomposition on the received signal without explicit channel estimation. The linear filter parameters are optimized by performing a Cholesky factorization of the correlation matrix. Although the motivation is triggered by the fiber-wireless uplink, the equalizer is effective against any general Wiener type nonlinear channel that consists of a time dispersive linear system followed by a memoryless static nonlinear system. These types of channels are frequently encountered in communication systems.

Following the remarkable work of N. Wiener, who first separated a nonlinear system into a linear memory part and a nonlinear memoryless part, we realize that separating the compensation of linear and nonlinear parts is more beneficial. This separated architecture has a special advantage when the linear dynamic system is a fast varying wireless channel. It enables updating the linear filter coefficients, leaving the nonlinearity compensation unchanged. This architecture is especially useful when multiple users share a single nonlinear link such as the fiber channel. In this case, the PLF can be kept common for all users and only the linear filters need to be different for each user. 


\section{Chapter 8}

\section{Performance Evaluation of the Hammerstein type DFE}

In Chapter 7, we proposed a Hammerstein type DFE (HDFE) for the concatenated fiber-wireless uplink. In this chapter the performance of the proposed HDFE is investigated. First, we investigate what happens to the inter symbol interference when it undergoes a nonlinear transformation. The HDFE attempts to reconstruct the linearity by performing another nonlinear transformation. We look at the statistical properties of the signal (and error) after this double transformation.

Then we evaluate the mean squared error of the PLF. There are two stages in the HDFE, the polynomial filter part and the DFE part. The mean squared error introduced in each of these parts are evaluated separately. The error at the PLF is a function of several parameters including, signal power, noise power, order of the channel polynomial $l$, order of the compensating polynomial $N$ and the dynamic range. On the other hand, the error in the DFE part is a function of signal to noise ratio and the length of the linear filters, especially the length of the feed forward filter.

\subsection{Evaluation of the Polynomial Filter}

There are two different errors occurring in the HDFE, the polynomial filter estimation error $\epsilon_{p}$ and the linear filter estimation error $\epsilon_{l}$. Of these, the first error $\epsilon_{p}$ occurs in the PLF between $q(n)$ and $z(n)$. This error $\epsilon_{p}=z(n)-q(n)$ is evaluated 
in this section.

We have shown in Chapter 6, that each separable Volterra kernel $w_{i}(n)$ is a function of the channel memory $L$ and the order of the compensating polynomial $N$ (expression 6.13). From equation (6.6) on page 114, the received signal $r(n)$ is a summation of $w_{i}(n)(1 \leq i \leq l)$. As a result, the mean squared error $J_{p}$ is a function of the order of the channel polynomial $l$, the channel memory $L$ and the order of the compensating polynomial $N$ as shown below.

$$
J_{p}(l, L, N)=E\left[\epsilon_{p}^{2}\right]=E\left[|z(n)-q(n)|^{2}\right]
$$

\subsubsection{Nonlinear Transformation of Time Dispersion}

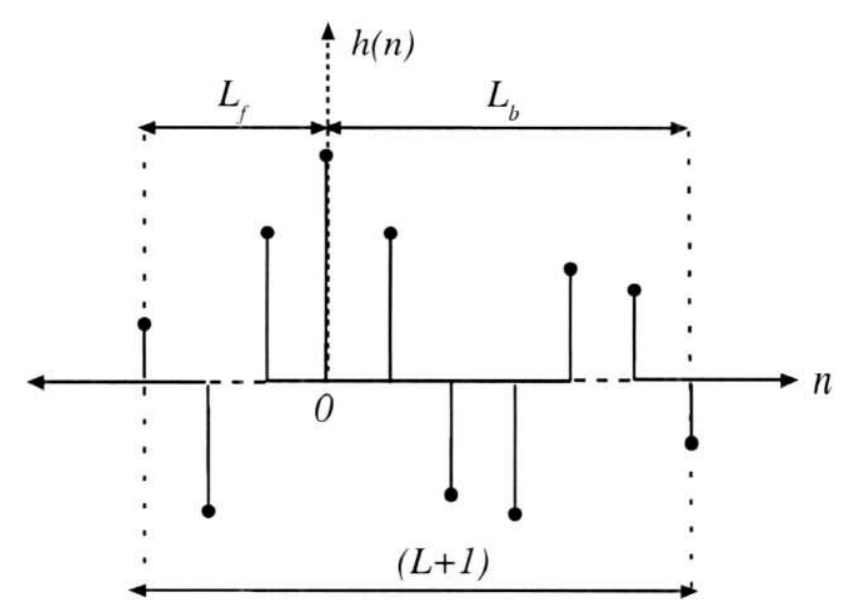

Figure 8.1: Typical impulse response of a wireless channel

Let us assume that the wireless channel has an impulse response as shown in Fig. 8.1. There are $L_{f}$ pre-cursors and $L_{b}$ post-cursors making a total number of $(L+1)=L_{f}+L_{b}$ cursors. Let $\{x(n)\}$ be an adequately long data sequence. Then, from equation (6.7), the first order kernel is just a linear convolution,

$$
w_{1}(n)=A_{1} \sum_{m=-L_{b}}^{L_{f}} h(m) x(n-m)
$$


This can be expanded as,

$$
w_{1}(0)=A_{1}\left\{x\left(-L_{b}\right) h\left(L_{b}\right)+\cdots+x(0) h(0)+\cdots+x\left(L_{f}\right) h\left(-L_{f}\right)\right\}
$$

Let the decision be made at the instance $n=0$. Then, the desired term is, $x(0) h(0)$ and there are $L$ interfering symbols. In other words, the received signal at any instance $n$ is a summation of $(L+1)$ terms. Typically, this is the case with linear channels. However, in our case, there are higher order terms. For example, the second order term is,

$$
w_{2}(n)=A_{2} \sum_{m_{1}=-L_{b}}^{L_{f}} h\left(m_{1}\right) x\left(n-m_{1}\right) \sum_{m_{2}=-L_{b}}^{L_{f}} h\left(m_{2}\right) x\left(n-m_{2}\right)
$$

which is expanded as,

$$
\begin{aligned}
w_{2}(0)= & A_{2}\left\{x^{2}\left(-L_{b}\right) h^{2}\left(L_{b}\right)+x^{2}\left(-L_{b}+1\right) h^{2}\left(L_{b}-1\right) \ldots+x^{2}\left(L_{f}\right) h^{2}\left(-L_{f}\right)\right. \\
& +2\left[x\left(-L_{b}\right) h\left(L_{b}\right) x\left(-L_{b}+1\right) h\left(L_{b}-1\right)+\ldots\right. \\
& \left.+x\left(L_{f}-1\right) h\left(-L_{f}+1\right) x\left(L_{f}\right) h\left(-L_{f}\right]\right\}
\end{aligned}
$$

If we assume $x(a) h(b)$ and $h(b) x(a)$ are two different terms for all $a$ and $b$ then there are $(L+1)^{2}$ terms due to the second order nonlinearity. Similarly,

$$
\begin{aligned}
w_{3}(n)= & A_{3} \sum_{m_{1}=-L_{b}}^{L_{f}} h\left(m_{1}\right) x\left(n-m_{1}\right) \sum_{m_{2}=-L_{b}}^{L_{f}} h\left(m_{2}\right) x\left(n-m_{2}\right) . \\
& \sum_{m_{3}=-L_{b}}^{L_{f}} h\left(m_{3}\right) x\left(n-m_{3}\right)
\end{aligned}
$$

and there are $(L+1)^{3}$ terms due to the third order nonlinearity again assuming $x(a) h(b)$ and $h(b) x(a)$ are two different terms for all $a$ and $b$. By expanding further, it can be shown that, the total number of interfering terms $N_{i s i}$ increases in a geometric series due to the nonlinear mapping of the dispersive channel. This series is shown below:

$$
N_{i s i}=L+(L+1)^{2}+(L+1)^{3}+\ldots+(L+1)^{l}
$$




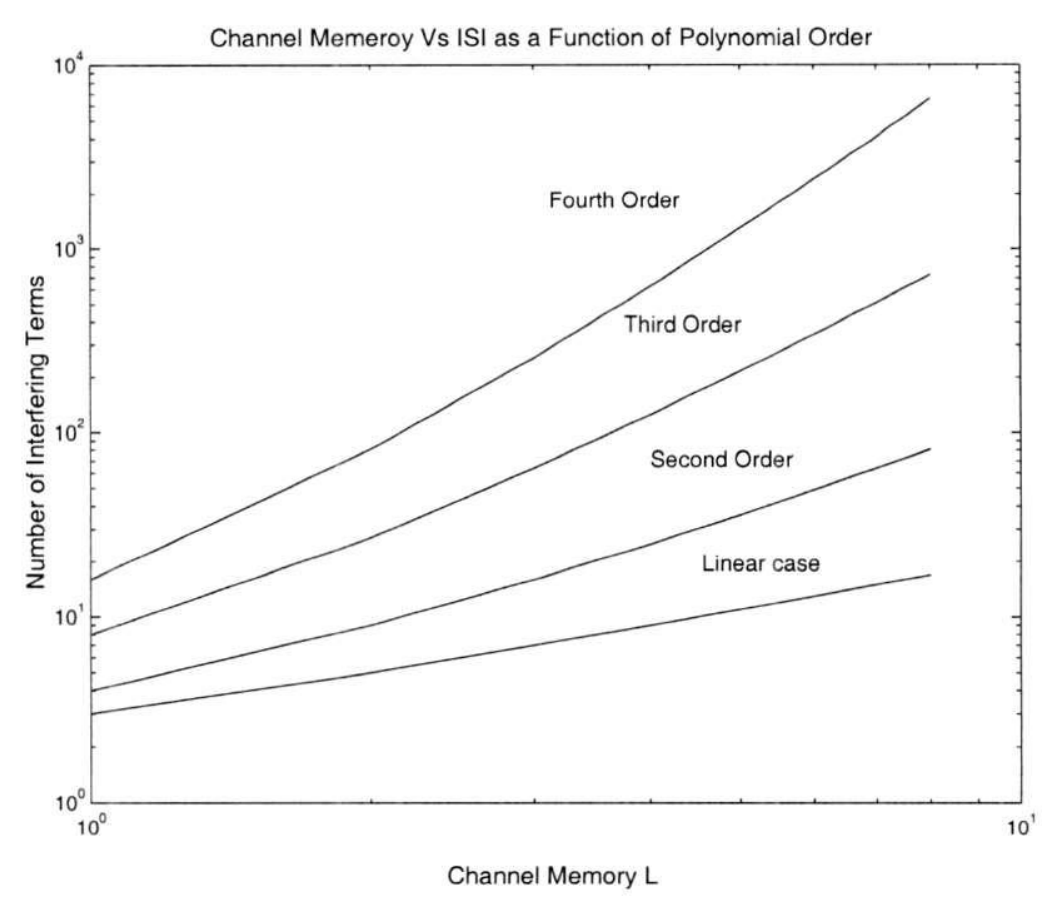

Figure 8.2: Inter symbol interference of a nonlinear dispersive channel

Fig. 8.2 shows, how the number of interfering terms increases with channel memory $L$, for different polynomial order $l$. For example, with a third order nonlinearity and a channel memory of eight, there are 818 interfering terms. This number spectacularly increases to 161051, with a fifth order nonlinearity and a channel memory of ten.

There is one more issue that needs to be addressed. Due to the nonlinear mapping, pre-cursor and post-cursor terms get cross-multiplied. This is clearly understood by expanding equation (8.6) and separating the desired term $x(0) h(0)$ as in equation (8.8). In addition, in equation (8.8), the pre-cursor and post-cursor terms also get multiplied with the desired term. Since, the success of the DFE resides in the fact that two different filters separately handle pre-cursors and post-cursor, these cross-multiplied terms would severely impair the performance, unless the polynomial filter adequately suppresses them. Furthermore, it is worth mentioning that out of these higher order interfering terms, even power terms are always positive and they contribute to an increase in the mean level of the signal, while the odd power terms can be either positive or negative. However, the even power terms 
are not usually a big concern because they fall at least one octave away from the passband.

$$
\begin{aligned}
& w_{3}(0)=A_{3}\left\{\sum_{m=-L_{b}}^{L_{f}} x^{3}(m) h^{3}(-m)\right. \\
& +3 x(0) h(0) \sum_{\substack{m=-L_{b} \\
m \neq 0}}^{L_{f}} x^{2}(m) h^{2}(-m) \\
& +3 x(0)^{2} h(0)^{2} \sum_{\substack{m=-L_{b} \\
m \neq 0}}^{L_{f}} x(m) h(-m) \\
& +3 \sum_{\substack{m_{1}=-L_{b} \\
m_{1} \neq 0}}^{L_{f}} x^{2}\left(m_{1}\right) h^{2}\left(-m_{1}\right) \sum_{\substack{m_{2}=-L_{b} \\
m_{2} \neq m_{1}, 0}}^{L_{f}} x\left(m_{2}\right) h\left(-m_{2}\right) \\
& +3 x(0) h(0) \sum_{\substack{m_{1}=-L_{b} \\
m_{1} \neq 0}}^{L_{f}} x\left(m_{1}\right) h\left(-m_{1}\right) \sum_{\substack{m_{2}=-L_{b} \\
m_{2} \neq m_{1}, 0}}^{L_{f}} x\left(m_{2}\right) h\left(-m_{2}\right) \\
& +\sum_{\substack{m_{1}=-L_{b} \\
m_{1} \neq 0}}^{L_{f}} x\left(m_{1}\right) h\left(-m_{1}\right) \sum_{\substack{m_{2}=-L_{b} \\
m_{2} \neq m_{1}, 0}}^{L_{f}} x\left(m_{2}\right) h\left(-m_{2}\right) \\
& \left.\sum_{\substack{m_{3}=-L_{b} \\
m_{3} \neq m_{1}, m_{2}, 0}}^{L_{f}} x\left(m_{3}\right) h\left(-m_{3}\right)\right\}
\end{aligned}
$$

\subsubsection{Inverse Polynomial Transformation}

The polynomial filter attempts to remove the nonlinear terms by performing another polynomial transformation. From Chapter 6, we know that $r(n)=w_{1}(n)+w_{2}(n)+$ $\ldots+w_{l}(n)+v(n)$. Now, the PLF output is given by $z(n)=g_{0}+g_{1} r(n)+\ldots+g_{N} r(n)$. If we substitute for $r(n)$ in terms of $w_{i}(n)$ and solve for $z(n)$, then the number of interfering terms $N_{l+N}$ at the output of the PLF forms another geometric series in terms of the original geometric series. The total number of terms in this double geometric series is a function of $L, l$ and $N$. The highest order of this series is $(N+l)$. Using the property that $w_{i}(n) \propto w^{i}(n)$ for $1 \leq i \leq l$ this series is expressed 


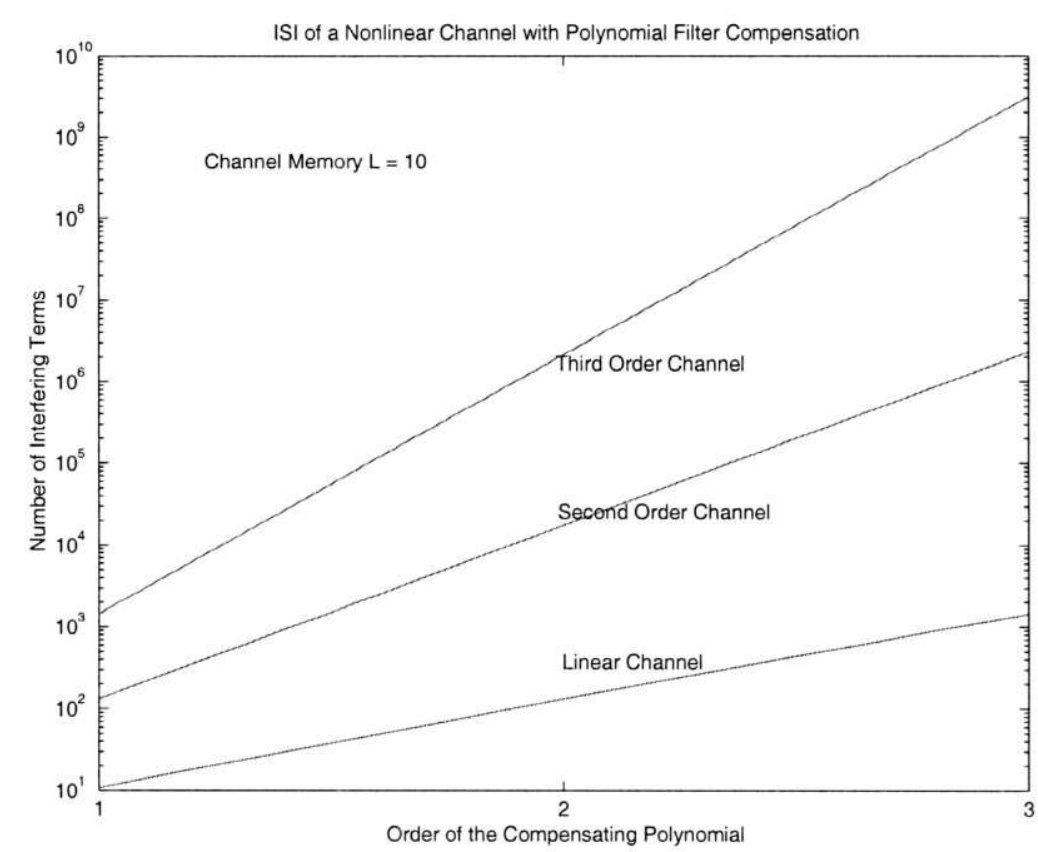

Figure 8.3: Number of interfering symbols after a double polynomial transformation

as,

$$
N_{l+N}=\sum_{i=1}^{N}\left\{\sum_{j=1}^{l}(L+1)^{j}\right\}^{i}
$$

assuming that $x(a) h(b)$ and $h(b) x(a)$ are two different terms for all $a$ and $b$. Fig 8.3 , shows the number of interfering symbols as a function of channel order $l$ and compensating polynomial order $N$, for channel memory $L=10$.

Consider the interference power, $P_{\text {int }}$, that is added to the desired symbol $x(0)$ from other symbols. We observe the following properties about this interference power:

1. The order of this double geometric series is $N+l$. Therefore, the $P_{\text {int }}$ consists of literally a huge number of terms. In case of $l \geq 3, N \geq 2$ and $L$ on the order of 10 , this corresponds to more than two million terms.

2. Each term in $P_{\text {int }}$ is a higher order, cross multiplied version of $x(n)$ and $h(n)$ $\left(-L_{b} \leq n \leq L_{f}\right)$, similar to the terms in equation (8.8). Therefore, there is a strong correlation between $P_{\text {int }}$ and the data $x(n)$. 
3. Because of the above properties, the correlation between $P_{\text {int }}$ and $h(n)\left(-L_{b} \leq\right.$ $\left.n \leq L_{f} ; n \neq 0\right)$ is also significant.

4. The power $P_{\text {int }}$ is much smaller than the signal power under practical conditions.

Finally, the output of the PLF, $z(n)$ is proportional to $q(n)$. Recall that $q(n)$ is the output of the linear channel $h(n)$ with only $L$ interfering terms. Therefore, the output of the polynomial filter, $z(n)$, consists of a sum of $L$ linear interfering terms. In addition, because of the nonlinear blocks, it has higher order interfering terms that have a power of $P_{\text {int }}$ due to the residual error $\epsilon_{p}$.

From the deductions above, we conclude that, the inter symbol interference power $P_{\text {int }}$ of a Wiener type nonlinear channel, which is proportional to $\epsilon_{p}$, is correlated with both the signal and the channel impulse response. However, according to the Weierstrass theorem, this power $P_{\text {int }}$ can be made arbitrarily small by selecting a BIBO stable polynomial filter with an appropriate order.

\subsubsection{Expected Value of the Polynomial Filter Error}

In this section we evaluate the mean square value of the polynomial filter error $\epsilon_{p}(n)=z(n)-q(n)$.

$$
J_{p}=E\left[\epsilon_{p}^{2}\right]=\overline{z^{2}(n)-2 z(n) q(n)+q^{2}(n)}
$$

Let us assume an independently and identically distributed (iid) data sequence $x(n)$. Furthermore, let us assume $x(n) \in\{-1,1\}^{1}$. For all $n$ with equal probability and no correlation between $x(n)$ and $x(m)$ for all $m \neq n$. Then,

$$
E[q(n)]=\sum_{m=-L_{b}}^{L_{f}} h(n-m) E[x(m)]=0
$$

Because of $x(n) \in\{-1,1\}$, then $x^{2}(m)=1$ for all $m$. By assuming that there is no

\footnotetext{
${ }^{1}$ any other amplitude is taken care by the factor $\alpha$ as shown in Chapter 6 .
} 
cross correlation between each path of the channel, we obtain

$$
E\left[q^{2}(n)\right]=\sum_{m=-L_{b}}^{L_{f}} h^{2}(n-m)
$$

Since, $x^{3}(m)=x(m) \times x^{2}(m)=x(m)$ and $E[x(m)]=0$ (equal probability property) we obtain,

$$
E\left[q^{3}(n)\right]=\sum_{m=-L_{b}}^{L_{f}} h^{3}(n-m) E\left[\left(x^{3}(m)\right]=0\right.
$$

Since, $E\left[x^{4}(m)\right]=E\left[x^{2}(m) \times x^{2}(m)\right]=1$

$$
E\left[q^{4}(n)\right]=\sum_{m=-L_{b}}^{L_{f}} h^{4}(n-m)
$$

Similarly, it can be shown that the expectation of all odd powers of $q(n)$ are zero. Expectations of even powers are given by the sum of corresponding higher order terms of the channel impulse response $h(n)$. This result is summarized as follows: Let us define a general symbol $\sigma_{h}^{i}$ as,

$$
\sigma_{h}^{i}=\sum_{m=-L_{b}}^{L_{f}} h^{i}(m)
$$

then,

$$
E\left[q^{i}\right]= \begin{cases}\sigma_{h}^{i} & \text { if } i \text { even } \\ 0 & \text { if } i \text { odd }\end{cases}
$$

where, $i$ is a positive integer. The timing notation $(n)$ is dropped in the above expression and hereafter, with an understanding that the whole manipulation is done at the discrete time instance $n$.

Now, consider the channel nonlinearity $r=\sum_{i=1}^{l} A_{i} q^{i}+v$. We need to consider only odd order terms because even order nonlinear terms generate harmonics that are at least one octave away from the carrier frequency. 
In this case, assuming $l$ is odd, $r$ is written as,

$$
r=\sum_{i=0}^{(l-1) / 2} A_{2 i+1} q^{2 i+1}+v
$$

Now, the expectations of $r$, when it is raised to higher powers, need to be analyzed. For this, consider a function,

$$
f(a)=\sum_{i=1}^{l} a_{i}
$$

Let us consider each term in $f^{n}(a)$, when $n$ is a positive integer. The power of each $a_{i}$ add to $n$ within each term, after raising $f(a)$ to the power $n$. Example terms are, $a_{i}^{n}, a_{i} a_{j}^{n-1}, \ldots a_{i}^{2} a_{j} a_{k}^{n-3}$ etc. Note that in each of these terms the total power is $n(n, 1+(n-1) \ldots 2+1+(n-3)$ etc. $)$. Because of this property, and since $r$ has only odd power terms of $q$, raising $r$ to an odd power results in weighted sums of only odd power terms of $q$.

Since, expectations of the odd power terms of $q$ are zero from (8.11) and (8.13), expectations of the odd power terms of $r$ are also zero.

$$
E\left[r^{i}(n)\right]=0 \quad i \in 1,3,5,7, \ldots, \infty
$$

Here, zero mean, signal independent noise is assumed.

To evaluate the even order expectations of $r$, first let us define the second order expectation $\sigma_{r}^{2}$ as,

$$
\sigma_{r}^{2}=E\left[r^{2}\right]
$$

Then from (8.17) and (8.16), for any positive odd integer $l$, the second order expectation $E\left[r^{2}\right]$ can be written as,

$$
E\left[r^{2}\right]=\sum_{i=0}^{(l-1) / 2} A_{2 i+1}^{2} \sigma_{h}^{4 i+2}+\sum_{i, j=0 ; i \neq j}^{(l-1) / 2} A_{2 i+1} A_{2 j+1} \sigma_{h}^{2 i+2 j+2}+\sigma_{v}^{2}
$$

where, $\sigma_{v}^{2}$ is the noise variance. For example, for $l=3(8.21)$ reduces to,

$$
\sigma_{r}^{2}=A_{1}^{2} \sigma_{h}^{2}+A_{3}^{2} \sigma_{h}^{6}+2 A_{1} A_{3} \sigma_{h}^{4}+\sigma_{v}^{2}
$$


Additional higher (even) order expectations for a given $l$ can be computed from the expression,

$$
E\left[r^{2 j}\right]=E\left[\left\{\sum_{i=0}^{(l-1) / 2} A_{2 i+1} q^{2 i+1}+v\right\}^{2 j}\right]
$$

where, $j$ is a positive integer. For example, for $l=3, E\left[r^{4}\right]=\sigma_{r}^{4}$ is given as,

$$
\sigma_{r}^{4}=A_{1}^{4} \sigma_{h}^{4}+A_{3}^{4} \sigma_{h}^{12}+4 A_{1} A_{3}^{3} \sigma_{h}^{10}+4 A_{1}^{3} A_{3} \sigma_{h}^{6}+6 A_{1}^{2} A_{3}^{2} \sigma_{h}^{8}+\sigma_{v}^{4}
$$

Note that the orders of $A_{i}$ add to 4 in each term in (8.24).

The next task is to compute the higher order expectations of $z=\sum_{i=1}^{N} g_{i} r^{i}$. Using the property in equation (8.19) and using the same approach that was used to find $E\left[r^{2}\right]$, it is straight forward to show that,

$$
E\left[z^{2}\right]=\sum_{i=1}^{N} g_{i}^{2} \sigma_{r}^{2 i}+\sum_{\substack{i, j=1 \\ i \neq j ; i+j=\text { even }}}^{N} g_{i} g_{j} \sigma_{r}^{i+j}
$$

Now, let us consider $z q=\sum_{i=1}^{N} g_{i} r^{i} q$. The task is to find $E[z q]=\sum_{i=1}^{N} g_{i} E\left[r^{i} q\right]$. For this, let us first find the expectation of $r q=r \times q$.

$$
r q=\sum_{i=0}^{(l-1) / 2} A_{2 i+1} q^{(2 i+2)}+v q
$$

Again with the assumption that $l$ is odd and noise is not correlated with the signal, we obtain

$$
E[r q]=\sum_{i=0}^{(l-1) / 2} A_{2 i+1} \sigma_{h}^{2 i+2}
$$

Note that, although $E[r]=0, E[r q] \neq 0$. However, for the second order, $E\left[r^{2} q\right]=0$, because it consists of only odd power terms of $q$. Similarly, all the even order expectations such as, $E\left[r^{4} q\right], E\left[r^{6} q\right]$ are zero. Odd order expectations can be computed from (8.28) where, $j$ is a positive integer.

$$
E\left[r^{2 j-1} q\right]=E\left[q\left\{\sum_{i=0}^{(l-1) / 2} A_{2 i+1} q^{2 i+1}+v\right\}^{2 j-1}\right]
$$


Finally, substituting all these results in equation (8.10) the mean squared error for the polynomial filter is given by,

$$
\begin{aligned}
J_{p} & =E\left[z^{2}\right]-2 E[z q]+E\left[q^{2}\right] \\
& =\sum_{i=1}^{N} g_{i}^{2} \sigma_{r}^{2 i}+\sum_{\substack{i, j=1 \\
i \neq j ; i+j=\text { even }}}^{N} g_{i} g_{j} \sigma_{r}^{i+j} \\
& -2 \sum_{i=1}^{N} g_{i} E\left[r^{i} q\right]+\sigma_{h}^{2}
\end{aligned}
$$

Note that $J_{p}$ is a function of channel impulse response $h(n)$, direct and inverse polynomial coefficients $A_{i}$ and $g_{i}$, and the noise power $\sigma_{v}^{2}$.

To understand the upper and lower bounds, we shall evaluate $J_{p}$ under the worst and the best conditions. Let $l=1$ and $N=1$, so that there is no nonlinearity. Then, taking $g_{1}=A_{1}=1$ and substituting in (8.29), $J_{p}=\sigma_{v}^{2}$. This is the best condition that provides a low bound for $J_{p}$. In all other cases $J_{p}>\sigma_{v}^{2}$. However, if $l>1, J_{p}$ is a function of $h(n)$ too. Upper bound of $J_{p}$ is encountered when the channel has a worst-case impulse response. This situation is discussed in Section 8.3.1.2.

\subsection{Evaluation of Linear Filters}

In the previous section we evaluated the mean squared error performance of the polynomial filter. In this section we look at the mean squared error performance of linear filters. Compared to the polynomial filter, performance evaluation of standard DFE with linear filters have been extensively studied [66], [80].

\subsubsection{Infinite Length Results}

Salz et al. [81] have shown that the minimum possible mean squared error of a DFE is achieved when it has infinite length filters. This MMSE, which serves as a performance bound for practical DFEs, is given as,

$$
M M S E_{\text {inf }}=\exp \left\{T_{c} \int_{-1 / 2 T_{c}}^{1 / 2 T_{c}} \ln \left[\frac{N_{o}}{|H(f)|^{2}+N_{o}}\right] d f\right\}
$$


where, $N_{o}$ is power spectral density of the complex additive white Gaussian noise and, $H(f)$ is the Fourier transform of $h(n)$ defined as,

$$
H(f)=\sum_{n=-\infty}^{\infty} h(n) e^{-j 2 \pi f n}
$$

\subsubsection{Finite Length Results}

In case of finite length filters, the MMSE is a function of filter length as well as delay. The MMSE of the DFE part, that has been optimized in Section 7.3.1, is given by the maximum entry in the diagonal matrix $\boldsymbol{D}$. However, the maximum entry is proven to lie between $N_{f-1}$ and $d_{N_{f}+L-1}$ [79]. Therefore,

$$
\begin{aligned}
M M S E & =\max \left\{d_{N_{f}-1}, d_{N_{f}}, \ldots, d_{N_{f}+L-1}\right\}^{-1} \\
& =d_{\Delta_{o p t}}^{-1}
\end{aligned}
$$

The optimum delay is numerically evaluated in Section 8.3.1.1. Generally, the MMSE is a function of both $N_{f}$ and $N_{b}$. However, when $N_{b} \geq L$ and there is no error propagation, the effect of post-cursors is negligible [50]. Hence, this error is a function of $N_{f}$ only.

\subsection{Numerical Results}

In this section the performance of the Hammerstein type DFE is evaluated numerically. This is done using the expression in (8.29). Note the MSE in (8.29) is a function of channel memory $L$, channel order $l$, compensation order $N$, higher order expectations of $h(n)$, and noise power. A suitable polynomial for the channel is assumed and the compensation polynomial is derived using the QR decomposition algorithm.

\subsubsection{Mean Squared Error of the Polynomial Filter}

In this section the MSE performance of the polynomial filter is evaluated. This is done considering two different channels. A measured channel with realistic multi- 


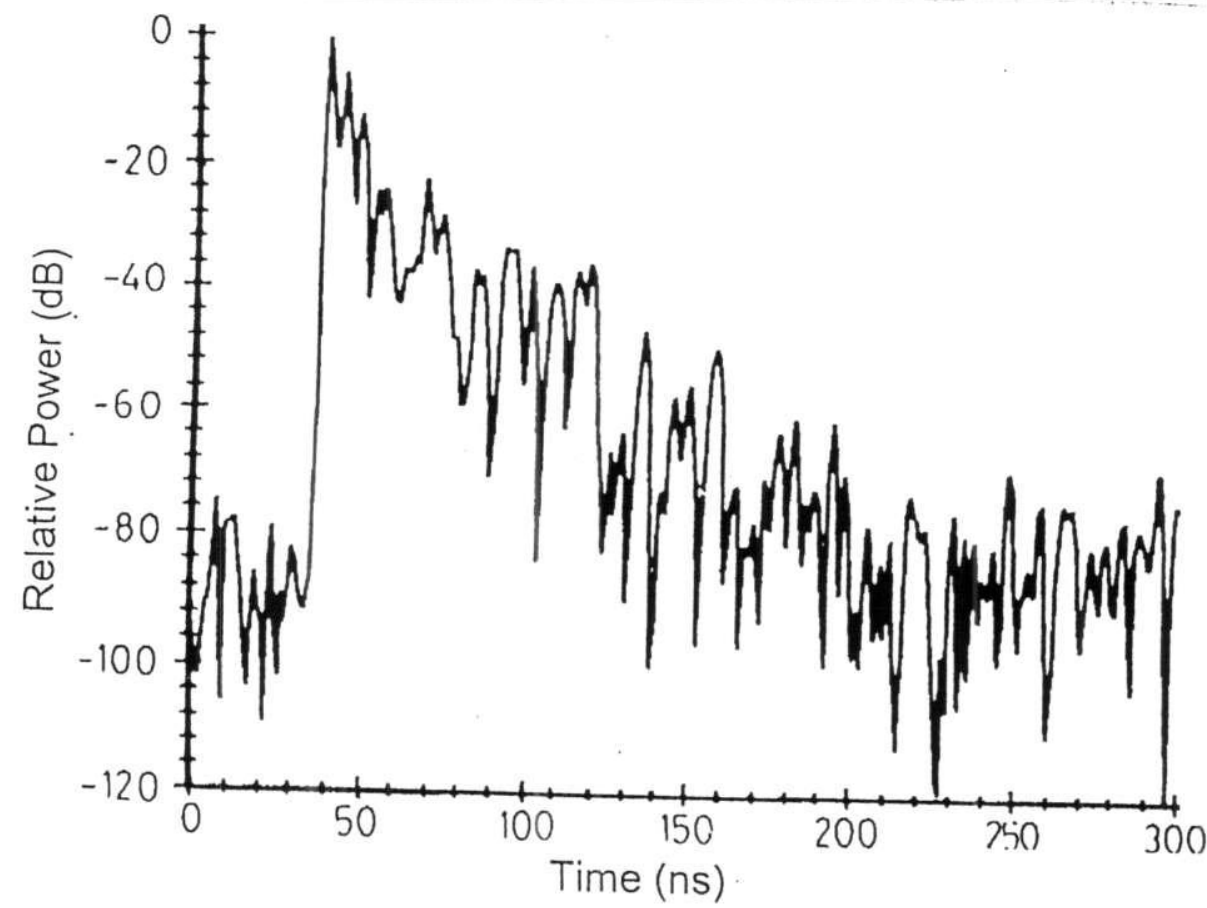

Figure 8.4: Measured impulse response at an indoor environment, courtesy [1]

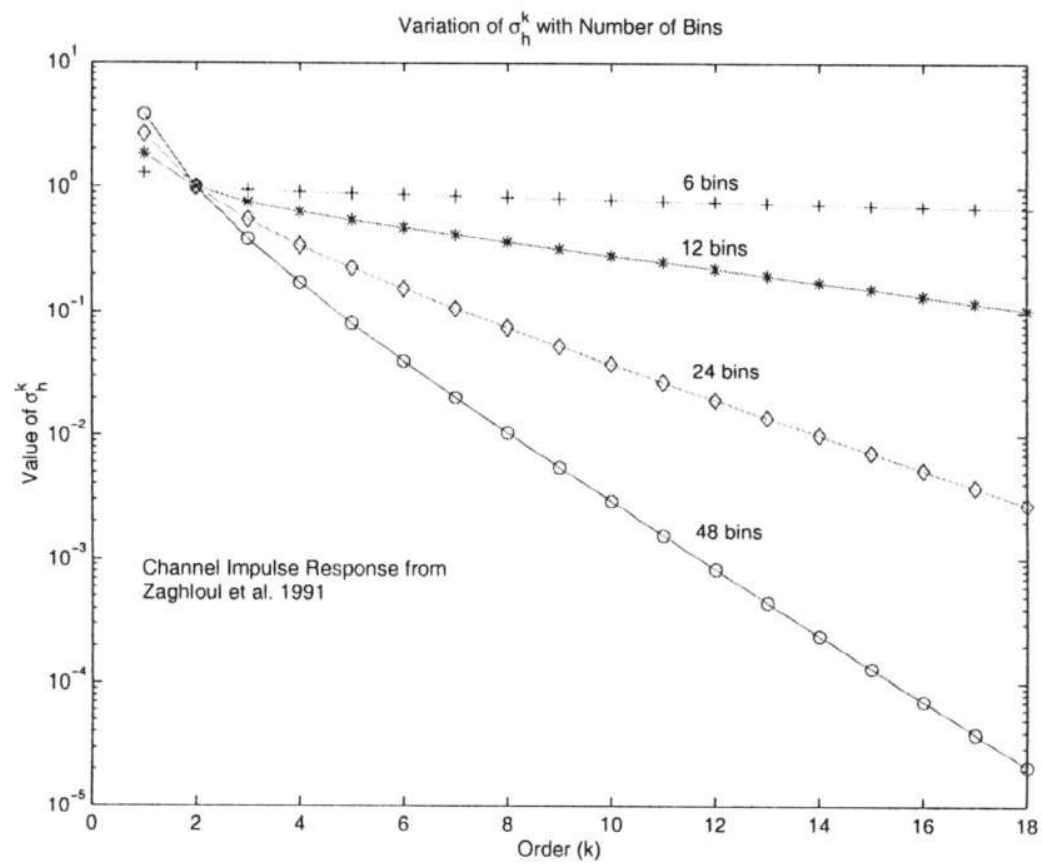

Figure 8.5: Higher order expectations of a channel that has impulse response shown in Fig. 8.4 


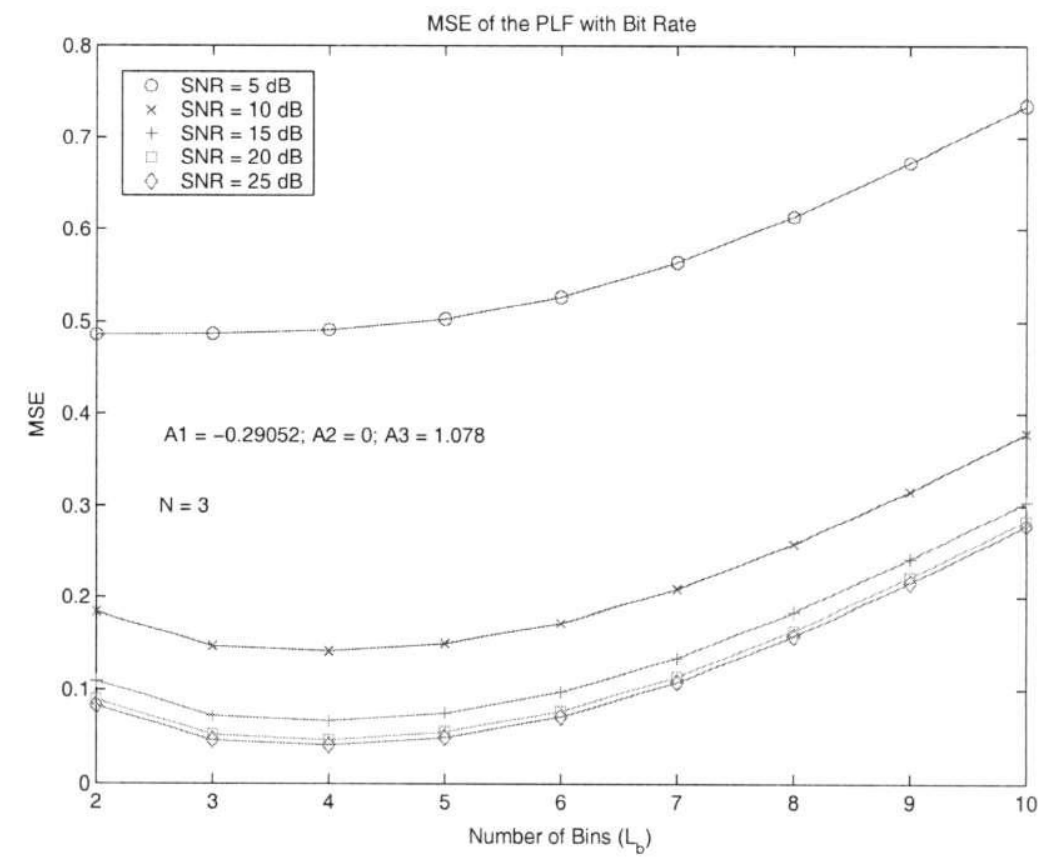

Figure 8.6: MSE of the polynomial filter versus number of bins $L_{b}$ for a third order compensation

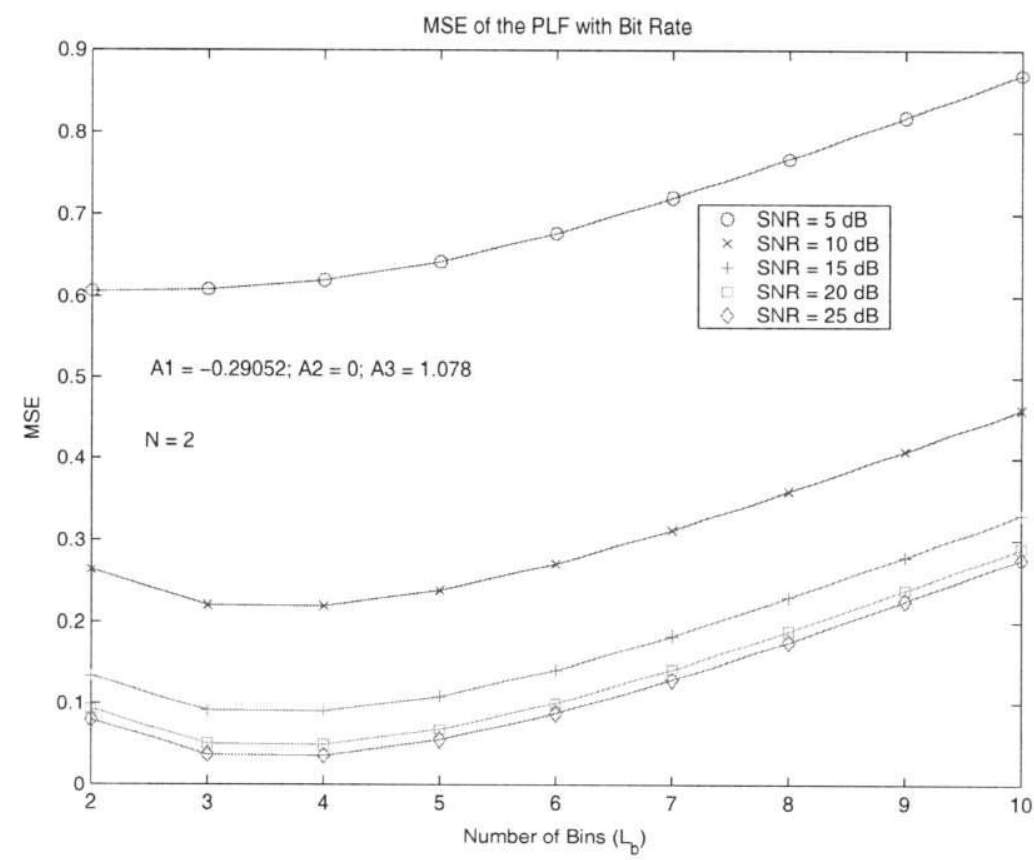

Figure 8.7: MSE of the polynomial filter versus number of bins $L_{b}$ for a second order compensation 


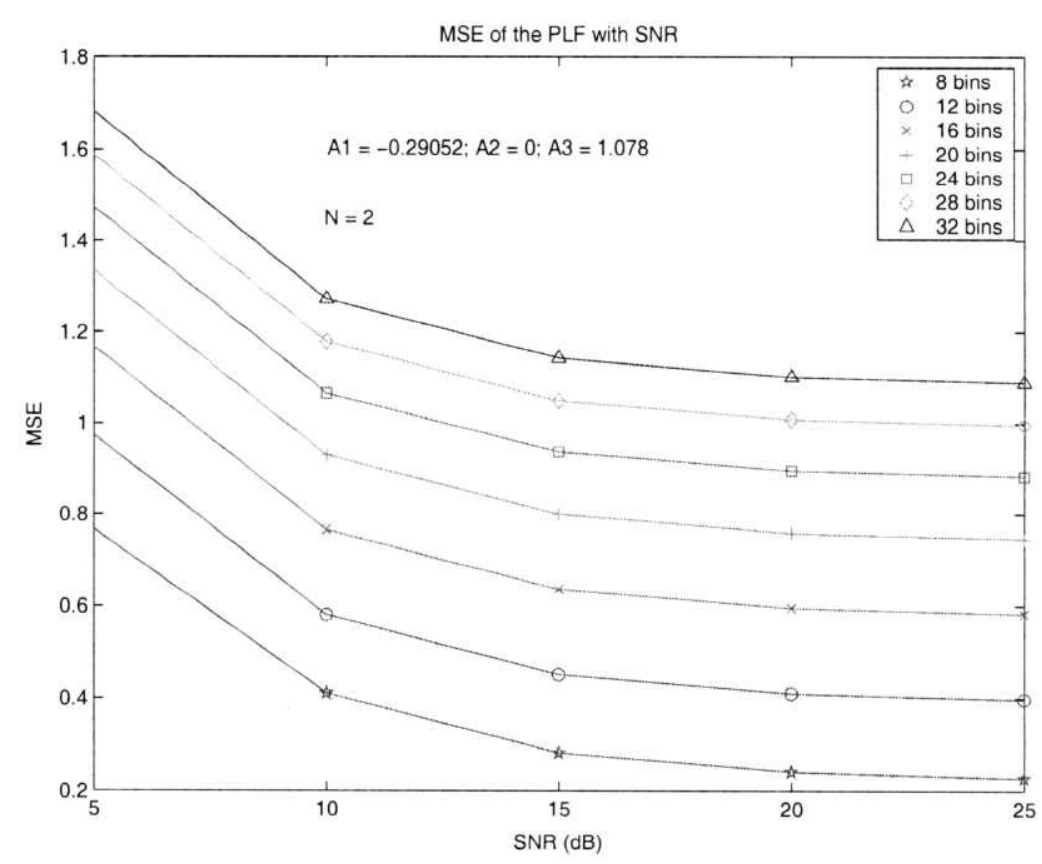

Figure 8.8: MSE of the polynomial filter versus SNR for a second order compensation

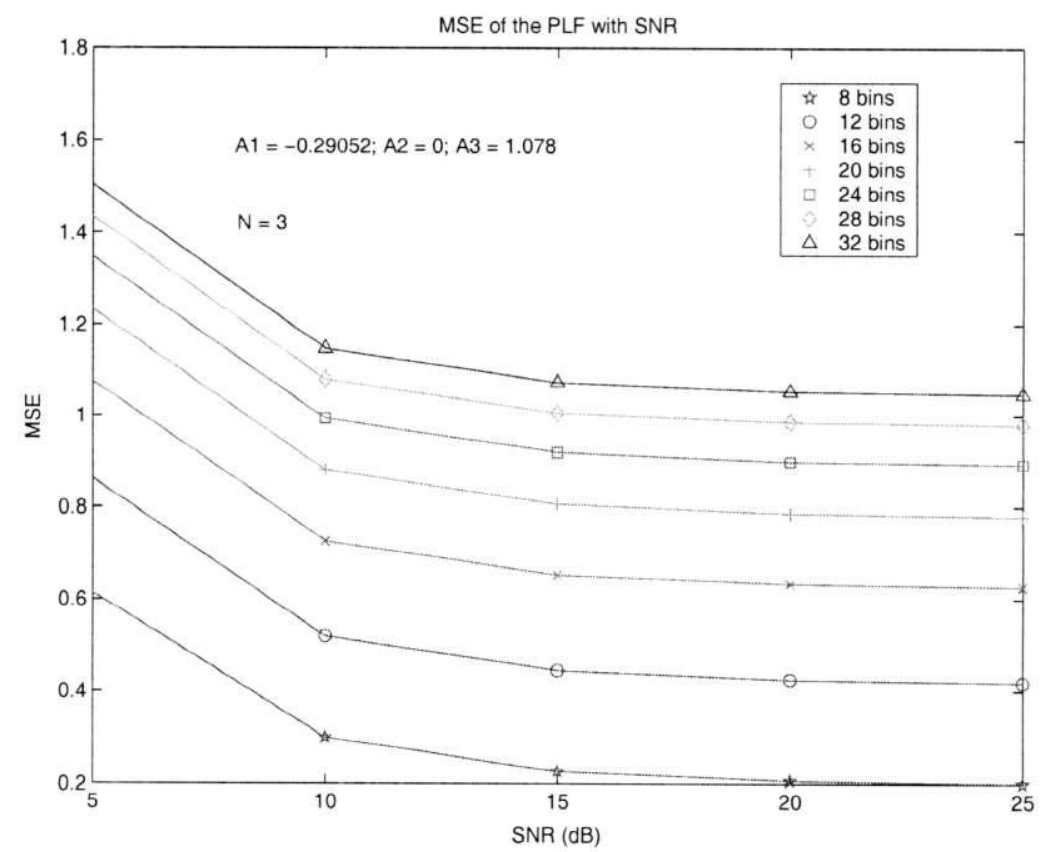

Figure 8.9: MSE of the polynomial filter versus SNR for a third order compensation 
path profile and an imaginary channel having all multipaths with equal strength. The first one gives a realistic evaluation while the second one gives a worst case evaluation.

\subsubsection{Real Channel Case}

The MSE of the polynomial filter is a function of channel impulse response $h(n)$, channel nonlinearity $l$, order of the polynomial filter $N$ and noise power $\sigma_{v}^{2}$. To evaluate this a measured impulse response in the indoor environment is used. The measurement was done on the third floor of the NovAtel ${ }^{T M}$ building at Calgary by Morrison et al. [1]. This impulse response (with a line of sight link) is shown in Fig. 8.4.

From the figure, the maximum delay is about $150 \mathrm{~ns}$. This is divided into $L_{b}$ number of bins. $L_{b}$ reflects the bit rate. For example, with a spreading of 32 , at $10 \mathrm{Mbps}$, the chip time is $3.125 \mathrm{~ns}$. Therefore, $L_{b} \approx 150 / 3.125 \approx 48$. Higher order expectations of the channel impulse response $\sigma_{h}^{k}$, (defined in Chapter 7 ), are shown in Fig. 8.5. From the figure it is seen that, at low bit rates (less number of bins) higher order expectations of the impulse responses are more significant. However, at high bit rates, (large number of bins), they decrease rapidly. This is because, as there are more resolvable paths, the normalized energy in each path becomes smaller so that, their higher order expectations become even smaller. However, this does not mean the MMSE is smaller, as we will see next.

Figures 8.6 and 8.7 show the MSE of the PLF as functions of SNR and $L_{b}$ for different orders of the compensating polynomial $N$. There is an optimum bit rate, proportional to $L_{b}$, at which the MSE is a minimum. Beyond this value, the MSE increases with $L_{b}$, even though $\sigma_{h}^{k}$ decreases with $L_{b}$ in Fig. 8.5. Furthermore, comparing these two figures, for the same SNR, a third order compensation $(N=3)$ provides less MSE than a second order $(N=2)$ compensation. This suggests that higher order compensation improves the MSE performance. This also agrees with our BER simulation curves, that are discussed later.

In addition, the MSE decreases with SNR in a nonlinear manner. This fact is explicitly visible in Figures 8.8 and 8.9. When the SNR is above $20 \mathrm{~dB}$, for example, there is very little decrease in the MSE. This is because, the limiting factor is no 


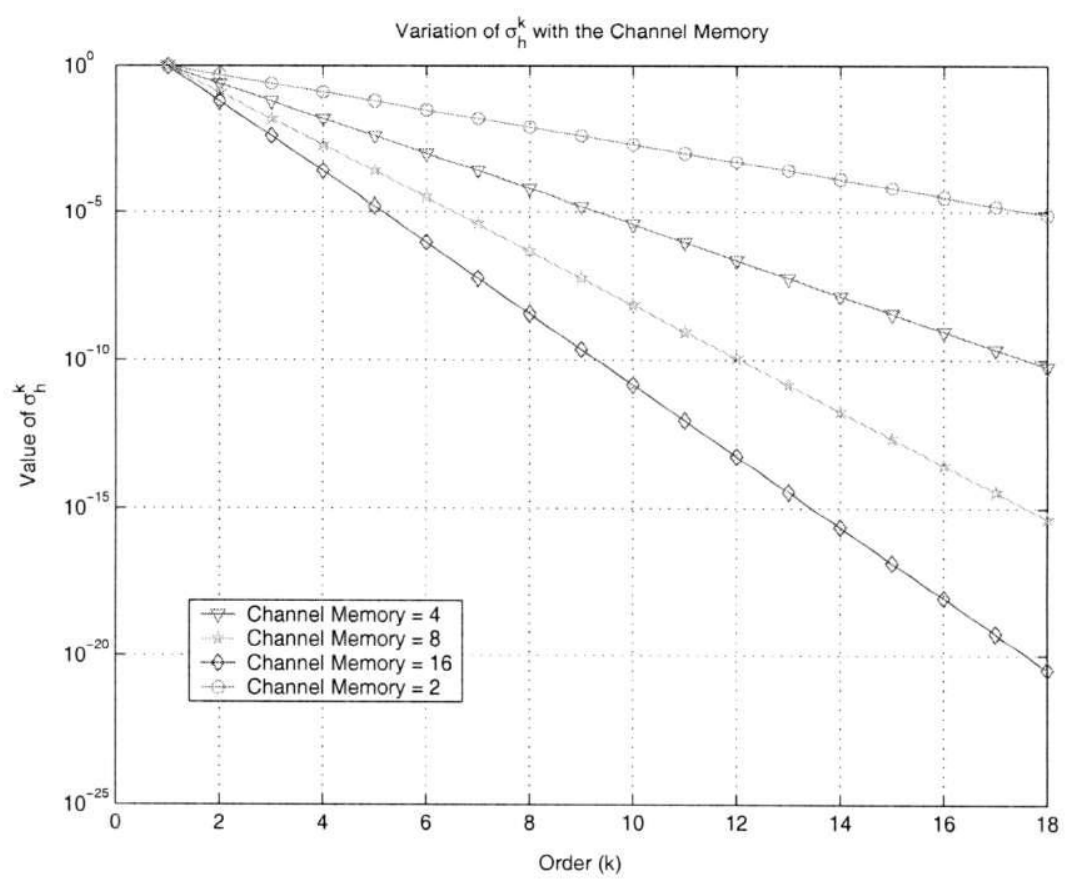

Figure 8.10: Variation of the parameter $\sigma_{h}$ with channel memory for the worst case channel

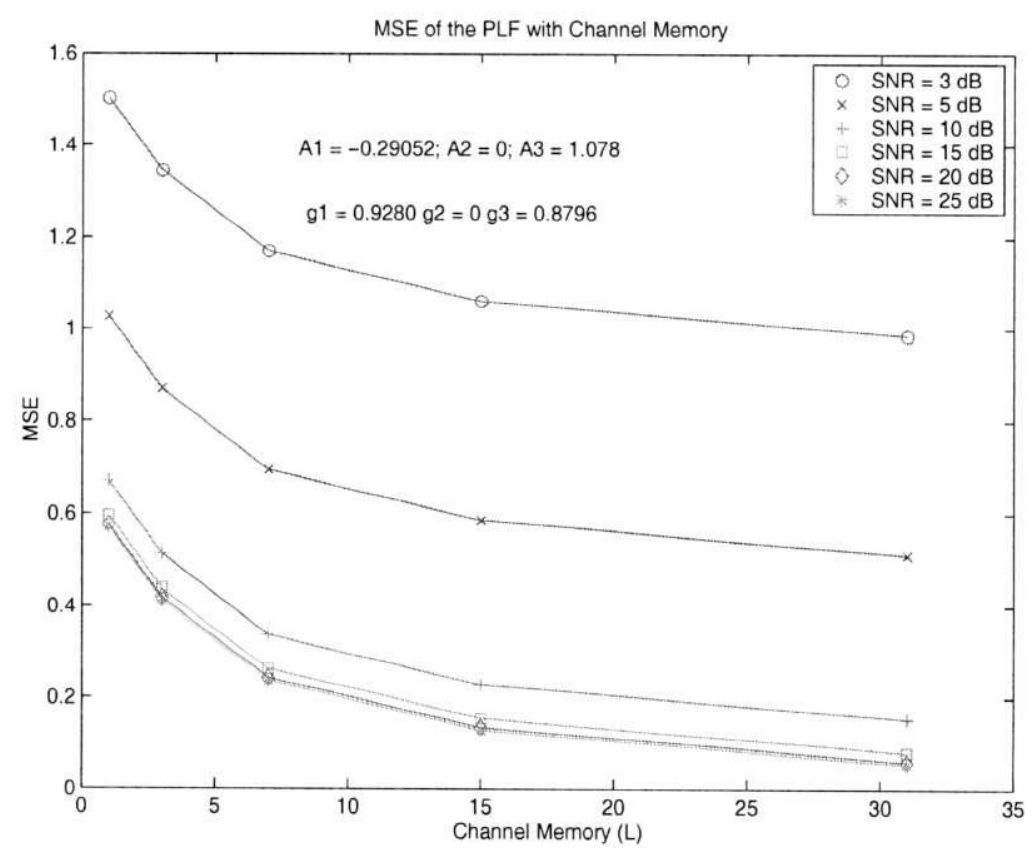

Figure 8.11: Mean squared error of the polynomial filter as a function of Channel Memory with a third order compensation for the worst case channel 


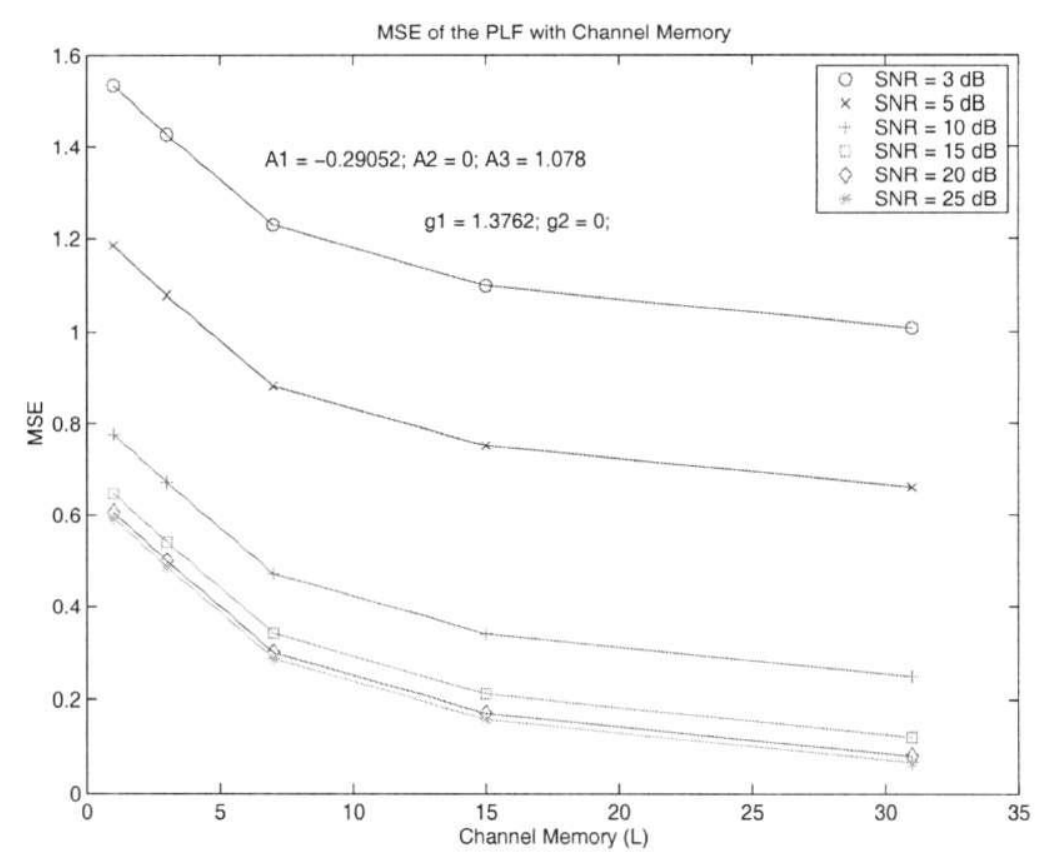

Figure 8.12: Mean squared error of the polynomial filter as a function of Channel Memory with a second order compensation for the worst case channel

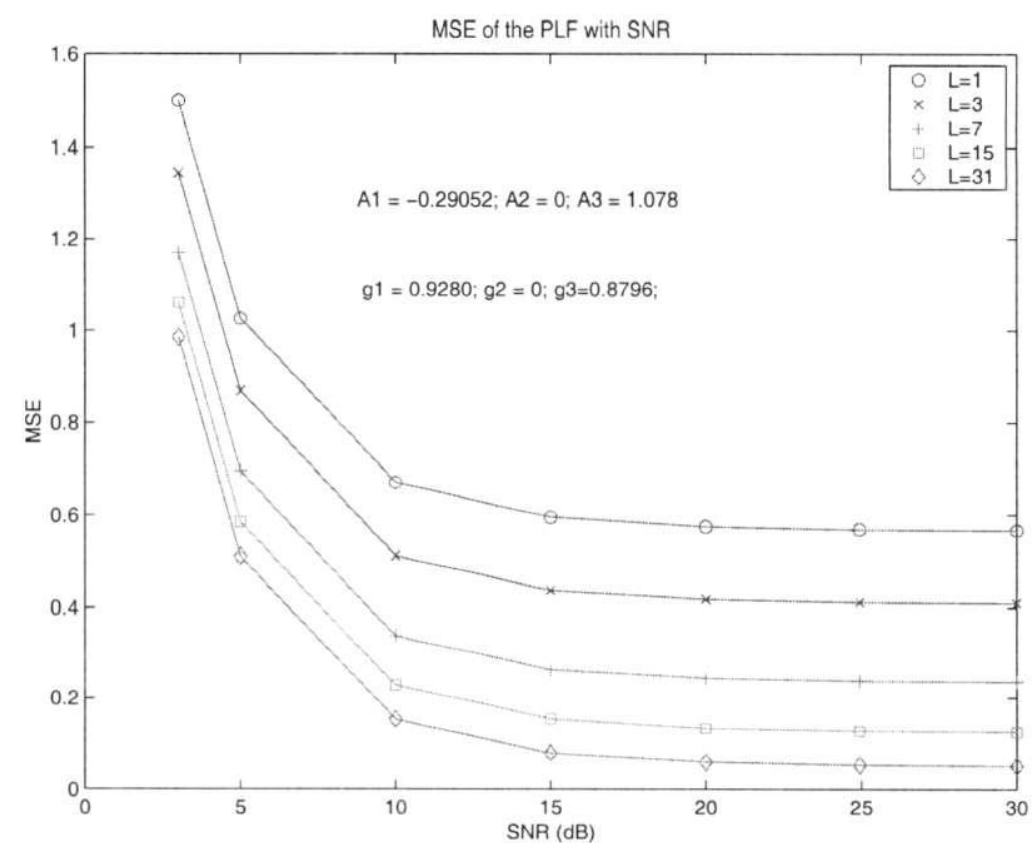

Figure 8.13: Mean squared error of the polynomial filter as a function of signal to noise ratio with a third order compensation for the worst case channel 


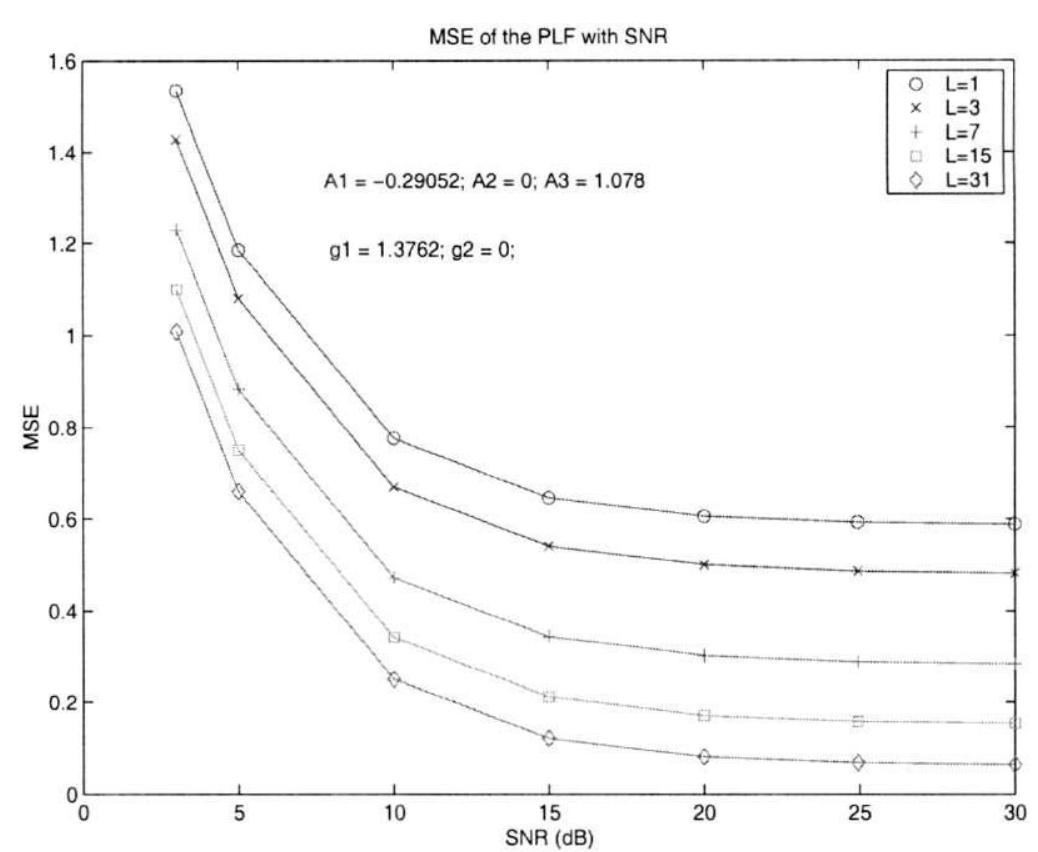

Figure 8.14: Mean squared error of the polynomial filter as a function of signal to noise ratio with a second order compensation for the worst case channel

longer the noise, but $L, l$, and $N$.

\subsubsection{Worst Case Channel}

For the worst-case evaluation we assumed a multipath channel with all paths having equal strengths. An impulse response with $(L+1)$ paths is defined by a normalized vector of dimension $(1 \times(L+1))$ as shown in (8.34). Note that memory of this channel is $L$.

$$
h(n)=\frac{1}{(L+1)}[1 ; 1 ; \ldots ; 1]_{(1 \times(L+1))}
$$

Higher order expectations of this worst case channel is shown in Fig. 8.10. We see that the higher order expectations rapidly diminish as the channel memory $L$ increases. Figures 8.11 and 8.12 show the mean squared error of the polynomial filter as a function of channel memory $L$ with third and second order compensations respectively. In contrast to the realistic channel, here we see that the MSE keeps decreasing as $L$ increases. However, the MSE saturates for large $L$. The reason for both of these phenomena is the same. The normalization makes the gain of each 

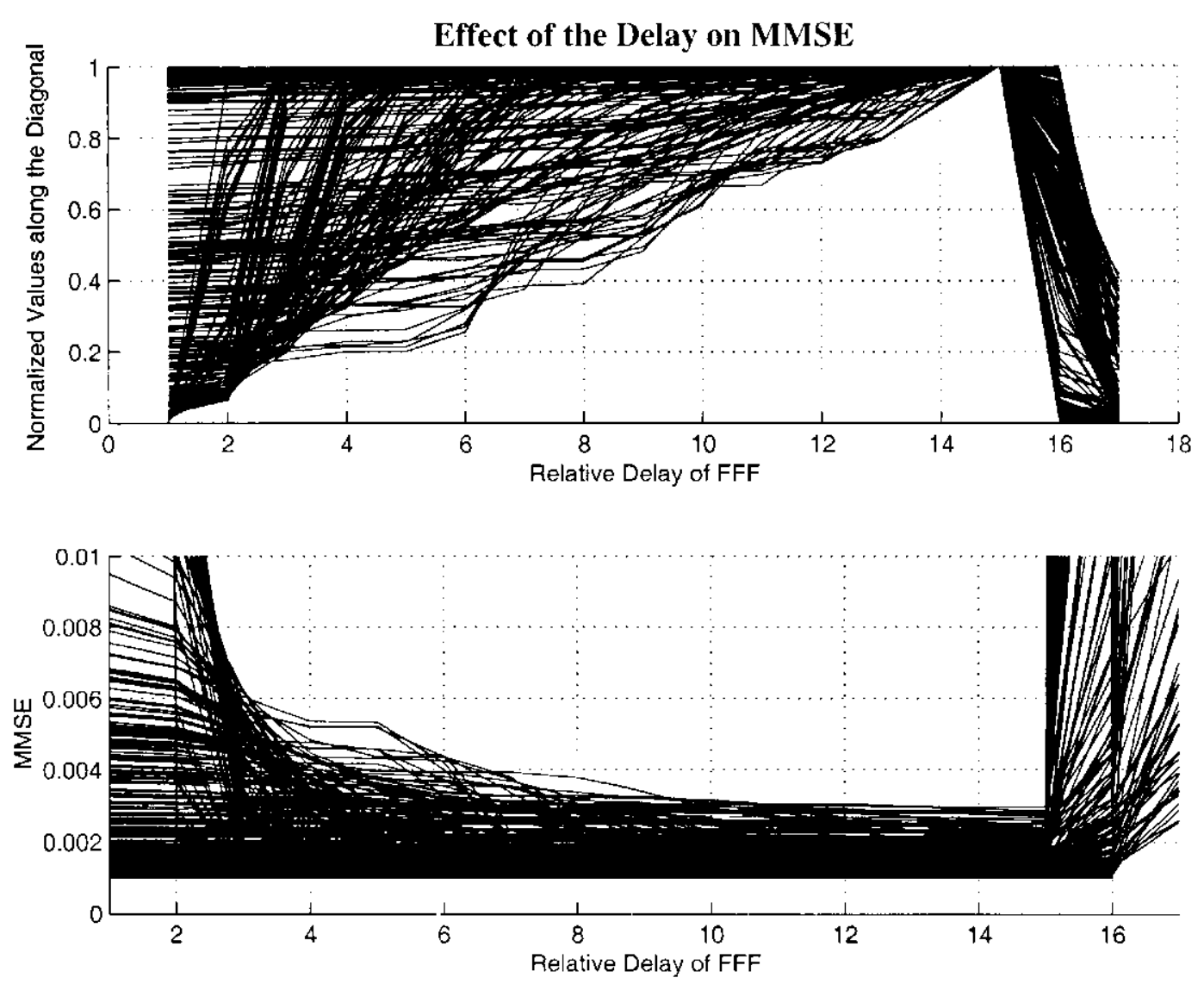

Figure 8.15: MMSE as a function of the feed forward filter delay

path $\left(\frac{1}{(L+1)}\right)$ to decrease as $L$ increases.

Figures 8.13 and 8.14 show mean squared error of the polynomial filter as a function of signal to noise ratio for the worst case channel with, third and second order compensations respectively. The MSE decreases with SNR in a nonlinear manner. When the SNR is above $20 \mathrm{~dB}$, for example, the MSE is almost flat. This is because, the limiting factor is no longer the noise, but $L, l$, and $N$. This phenomenon is common for any type of channel.

Mean squared error of the linear filters is given in (7.45) in Chapter 7. This is a function of delay $\Delta$ provided, that the FFF has adequate length and the $N_{b}=L$, In general, the optimum delay $\Delta_{\text {opt }}$ lies between, $\left(N_{f}-1 \leq \Delta_{\text {opt }} \leq N_{f}+L-1\right)$. The optimum delay correspond to the maximum entry in $D$. To determine the exact value of $\Delta_{o p t}$ we performed a simulation and found that the optimal delay of the HDFE is $N_{f}-1$. Such a typical simulation result is shown in Fig. 8.15 
where, the peak of the normalized values along $D$ happens at a delay of 15 . This coincides with the delay for the minimum MSE. We assumed an $N_{f}$ of 16 for the simulation. This is in agreement with the results obtained by Cioffi et al., who showed by simulation that $N_{f}-1$ is the optimum delay with adequately long feed forward filters. Furthermore, when the delay is optimal, the feedback filter weights closely resemble the post-cursors of the channel impulse response. This is shown in Fig. 8.16.

\subsubsection{BER Performance of the HDFE}

Finally, the BER performance of the HDFE is shown in Fig. 8.17. The lowermost curve is the performance of the basic DFE in a linear channel with similar FFF and FBF filter parameters. The other three curves are obtained under nonlinear channel conditions with a channel polynomial of $r(n)=-0.29052 q(n)+1.078 q^{3}(n)$. A Rician fading channel is simulated with a dominant line of sight path, and four more fading paths so that $L=4$. The lengths of the linear filters are taken as $N_{b}=L$ and $N_{f}=5 L$. The $K$ factor of the Rician channel is taken as 0.4. Doppler spread is not considered. Simulink ${ }^{T M}$ dynamic system simulator running in Matlab ${ }^{T M}$ environment is used for simulation.

\subsubsection{Mean Squared Error of Linear Filters}

It shows that with adequate compensation, (when a third order channel is compensated with a fifth order PLF), the performance of the HDFE is close to that of a DFE in a linear channel. It approaches the linear DFE performance at higher SNR. This is because; both the nonlinear distortion and noise affect the performance of the HDFE at low SNR. When a second order HDFE is used, performance degrades. However, at higher SNR, the second order HDFE shows much better performance than the standard DFE. The DFE exhibits an irreducible BER floor at higher SNR, because of the NLD. 

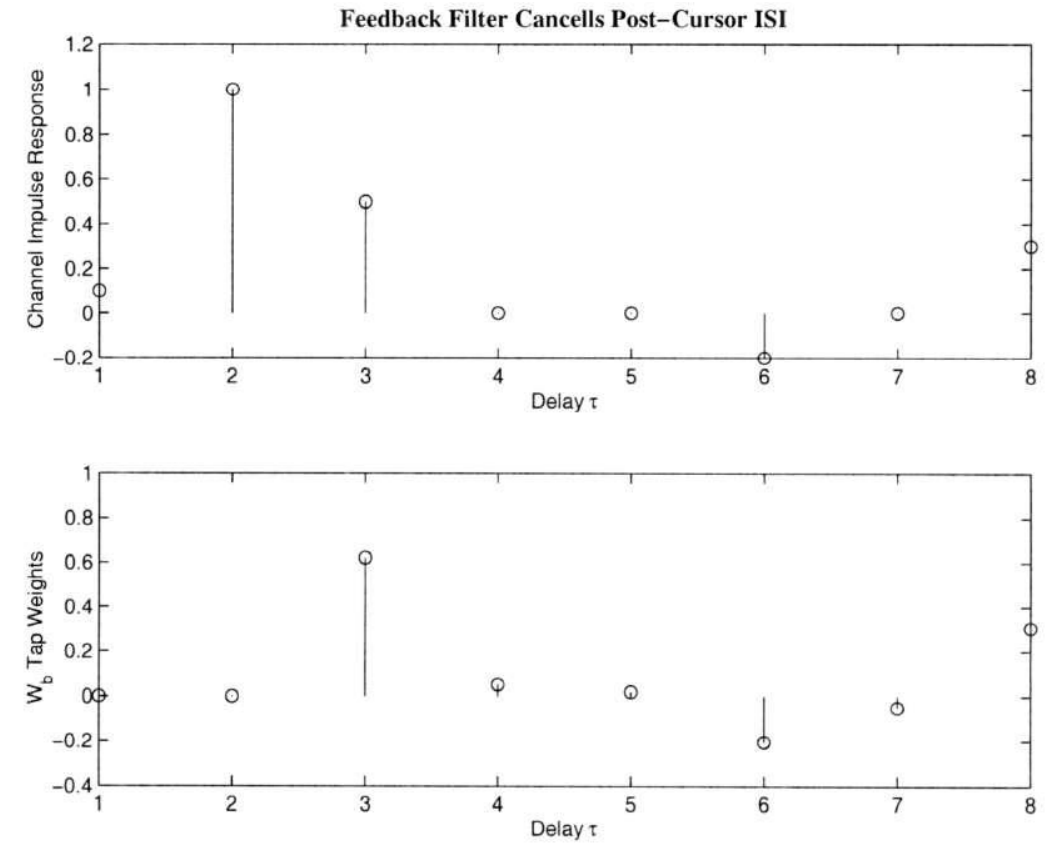

Figure 8.16: Weights of the feedback filter that is compared to the channel postcursors

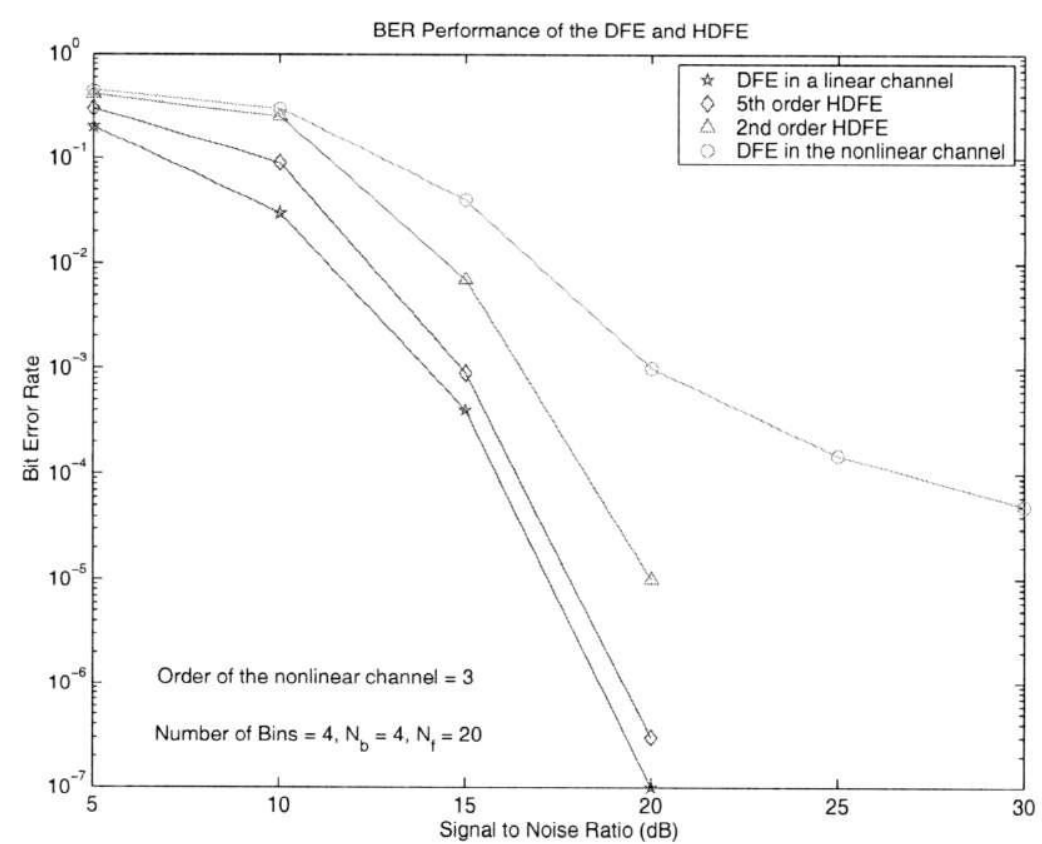

Figure 8.17: Bit error rate performance of the HDFE and a standard DFE 


\subsection{Concluding Remarks}

In this chapter we have analyzed the performance of the HDFE. First, we showed that when a multipath channel is followed by a nonlinear system, the number of interfering symbols rapidly increases. This depends on channel memory and nonlinearity. We also examined the statistical properties of the ISI terms after the nonlinear transformation.

For performance evaluation, we derived an expression for the mean squared value of the polynomial filter estimation error $J_{p}$. Its lower bound is the noise power. Its typical values depend on higher order expectations of the channel impulse response $\sigma_{h}^{k}$ and polynomial filter coefficients $g_{i}$ and order $N$.

We numerically evaluated this MSE using a real and a worst case channel and found that there is an optimum bit rate $L_{b}$ at which the MSE is a minimum (Figures 8.6 and 8.7). Another useful observation is that this optimum $L_{b}$ is independent of the SNR and order of compensation. It depends mainly on the impulse response of the particular channel.

Finally, the BER performance of the HDFE shows that with adequate compensation - a third order channel is compensated with a fifth order PLF - the performance of the HDFE is very close to that of a DFE in a linear channel under similar FFF and FBF filter parameters. 


\section{Chapter 9}

\section{Conclusions and Suggestions for Future Work}

Signal processing approaches to improve the performance of microwave fiber-optic links is in its infancy, especially in the case of wireless applications. There is plenty of work to be done before a widespread deployment of fiber based wireless schemes become commercially feasible. For example, recently a number of demonstrations have been done on fiber based wireless links as outlined in [9] and [21]. The concern is that most of them are confined to the laboratory level. Both small (Eg. Magis networks) and big (Eg. British Telecom.) companies still struggle to make profit from this technology. The major reason for this is lack of robustness. All those prototypes have been accomplished with careful design procedures and perfect finetuning by photonic experts. Reproducing those on a mass scale for day-to-day usage is still too costly.

To make this technology available for the public in a cost-effective manner and for the industry to make revenue, the ROF link should be integrated as a part of the wireless network. For this, robust deployment techniques are mandatory. Ultimately, the deployment of a fiber based wireless scheme should use off-the-shelf devices and the performance of the link should be independent of the optical link parameters. Adaptive-signal-processing techniques will play a major role to provide this kind of solution. In this dissertation, we have just initiated such a process. 


\subsection{Major Conclusions}

In this section, major conclusions of this dissertation are summarized. References are made to previous chapters wherever appropriate. Then, in the next section, some suggestions are made to outline identified future research directions. Being a relatively new topic, there is plenty of room for research in this optical-wireless area.

\subsubsection{Characterization and Compensation}

In this dissertation, we investigated signal-processing strategies to improve the performance of a fiber based wireless access scheme. For this, we first characterized the radio over fiber link both theoretically and experimentally. From this, we found that when the radio frequency is only a few $\mathrm{GHz}$ and the fiber length is a few kilometers, fiber dispersion is negligible. AM-AM and AM-PM type nonlinear distortion of the link, however, significantly limits the performance. Therefore, we focus on compensating for the AM-AM and AM-PM type nonlinear distortions. Typically, $\mathrm{E} / \mathrm{O}$ conversion process is the major source of the nonlinear distortion. In wireless applications, however, high power radio amplifiers (HPA) introduce additional nonlinear distortion, which is significant.

An investigation is then done on adaptive nonlinearity compensation schemes assuming an AWGN wireless channel. Predistortion is done for the downlink and post compensation is done for the uplink. This provides cost-effective asymmetrical arrangement because most of the signal processing is done at the central base station. Both phase and amplitude nonlinearities are handled individually. Simulation based on measured data shows adequate compensation is achieved under the following conditions:

1. Amplitude post-compensation works well with a small power back-off.

2. Phase post-compensation works well when the restored amplitude is used for estimating the phase error. That is, when the phase post-compensation filter follows the amplitude post-compensation filter (see Fig. 4.12).

3. Amplitude pre-Compensation needs a considerable $(\approx 30 \%)$ power back-off 
due to the power handling capability of the laser.

4. Phase pre-Compensation works well since the distortion is a function of input power and the filter uses the same input power for estimation

\subsubsection{Noise and BER Analysis}

Next, the BER performance of the linearized ROF link and an AWGN wireless channel is analyzed. This shows, when the optical modulation depth $m$ is significantly high, the relative intensity noise (RIN) increases with $m$ as shown in (5.22). Generally, this effect is ignored because of the small $m$. With nonlinearity compensation schemes, however, $m$ can be large and the contribution of $m^{2}\left\langle s^{2}(t)\right\rangle$ term is significant. From the derived expression for SNR, which is repeated here for convenience, we have the following conclusions:

$$
S N R=\frac{m^{2} I_{D}^{2} E\left[s^{2}(t)\right]}{\left(2 q I_{D}+(R I N)_{l i n} I_{D}^{2}\left[1+m^{2} E\left[s^{2}(t)\right]\right]+4 F K_{B} T_{o} / R_{L}\right) B}
$$

1. The higher the bandwidth $B$ of the SS signal $s(t)$, the lower the SNR. However, at the portable receiver, when the SS signal is despread, wider bandwidth means more processing gain. Therefore, there is an optimal bandwidth $B$ for a given number of users and the ROF link.

2. With nonlinearity compensation schemes, the modulation index $m$ can be made higher (note that usually $m<0.3$ ). Higher $m$ yields better SNR in the ROF link.

3. In the shot noise limited case $S N R=m^{2} I_{D} E\left[s^{2}(t)\right] / 2 q B$. That is, SNR increases with mean detected current $I_{D}$. The mean detected current is proportional to the mean optical power $P_{o}$. However, large $P_{o}$ means low $m$. Therefore, there is an optimum $m$ in the shot noise limited case.

4. In the RIN limited case, $S N R \approx m^{2} E\left[s^{2}(t)\right] /(R I N)_{l i n} B$. That is, the SNR is independent of mean optical power and increases with the RF power. However, when the RF power is large enough $\left(m^{2} E\left[s^{2}(t)\right]>1\right)$ then, the SNR saturates. 
5. The signal is weakest at the optical receiver as well as at the wireless receiver.

Therefore, the optical channel noise is the dominant noise when the portable is very close the RAP, that is, when the wireless channel loss $\left(L_{w l}\right)$ is smaller the than the optical receiver amplifier gain $\left(G_{o p t}\right)$. If it is the other way around, then the wireless channel noise is dominant.

\subsubsection{Estimation of the Fiber-Wireless Channel}

For a more realistic solution the multipath dispersion of the wireless channel should also be considered, in addition to nonlinear distortions. In Chapter 6, we extended the work to estimate the concatenated fiber-wireless channel using autocovariance properties of PN sequences. The algorithm estimates both the linear part and the nonlinear part of the concatenated channel individually.

It is important to mention that the shape of the nonlinearity is immaterial for the algorithm to work. It always identifies the exact curve, provided there is a one to one relationship. Simulation results, using real data, show excellent estimation with just a few training symbols. Ideally (when there is no noise), one symbol is enough for identification.

The length of the training sequence is independent of the order of the nonlinearity $l$. However, the sample points need to span the whole dynamic range of interest. It is interesting to see that this is better achieved when the wireless channel has severe multipath conditions. Resolution of the impulse response depends on the chip time because, the sampling is done at the chip rate. However, the memory of the linear channel can be as long as the length of the PN sequence $\left(L \leq N_{c}\right)$.

\subsubsection{A Hammerstein type Decision Feedback Equalizer for the Uplink}

As our major contribution, we have proposed a Hammerstein type decision feedback equalizer for compensating Wiener type nonlinear channels. Although the motivation is triggered by the fiber-wireless uplink, the equalizer is effective against any general Wiener type nonlinear channel that consists of a time dispersive linear system followed by a memoryless static nonlinear system. These types of channels are 
frequently encountered in communication systems.

Following the remarkable work of N. Wiener, who first separated a nonlinear system into a linear memory part and a nonlinear memoryless part, we realized that separating the compensation of linear and nonlinear parts is more beneficial. This separated architecture has a special advantage when, the linear dynamic system is a fast changing wireless channel. It enables updating the linear filter coefficients independent of the nonlinearity. Furthermore, this architecture is especially useful when multiple users share a single nonlinear link such as the fiber channel. In this case, the PLF can be kept common for all users and only the linear filters need to be different for each user.

We developed a fast optimization procedure for the filter parameters. The polynomial filter parameters are directly optimized by performing QR decomposition on the received signal. Linear filter parameters are optimized by performing a Cholesky factorization.

The BER performance of the HDFE shows that with adequate compensation, (when a third order channel is compensated with a fifth order PLF), the performance of the HDFE is very close to that of a DFE in a linear channel under similar FFF and FBF filter parameters. The effectiveness of the compensation relies on the accuracy of the channel estimation, the ability to find a finite order inverse polynomial within the desired dynamic range and, on the validity of the memoryless assumption to the nonlinearity.

\subsubsection{Separate Predistortion and Equalization for the}

\section{Downlink}

Although, the nonlinear distortion is not severe in the fiber-wireless downlink compared to the uplink, phase nonlinearity is a major concern in the downlink. Furthermore, the RF power fluctuates depending on the number of users and, the signal envelope is not constant (typically described by peak to average ratio). Therefore, we propose an adaptive pre-compensation scheme for the downlink, which is our secondary contribution. Details of this predistortion scheme are given in Chapter 4. The predistortion filter handles only the nonlinear distortion. The wireless channel dispersion is handled by the portable receiver using a suitable equalizer, for 
The Channel

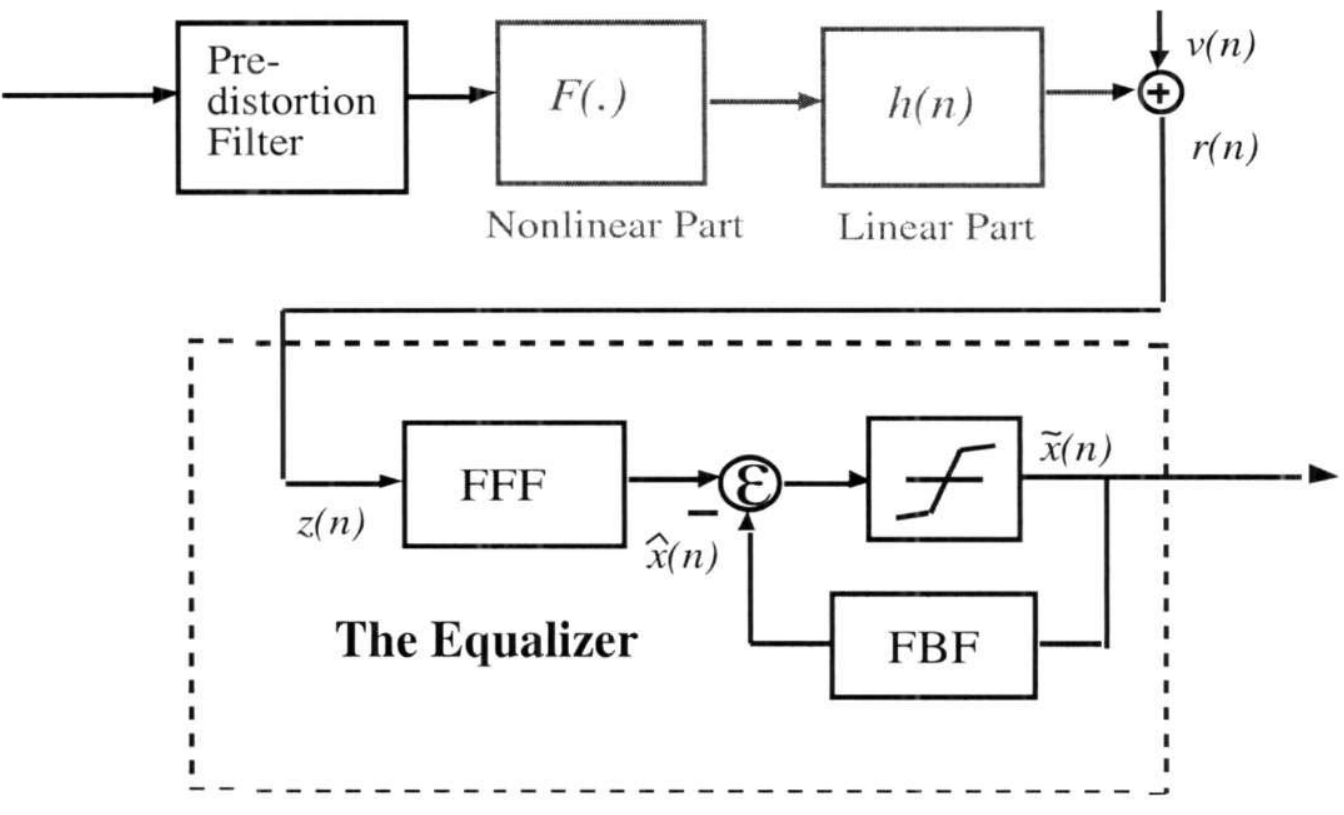

Figure 9.1: Downlink compensation arrangement

example, a decision feedback equalizer. Such an arrangement is shown in Fig. 9.1.

A major advantage of this asymmetric solution is that the portable unit is not aware of the ROF link. There are no modifications done at the portable unit, because of the ROF link. That means seamless roaming between fiber based and conventional wireless networks is possible. This is beneficial, not only to manufacturers but also to service providers.

\section{$9.2 \quad$ Future Directions}

We identify that in the short term, the following specific issues need to be addressed. Additional issues will become clearer as the research progresses further.

\subsubsection{Compensation Related Issues}

In this section, we first look at the issues that are specific to the adaptive compensation scheme. Then, we look at the additional ROF link issues, which we did not address in this work. Finally, we review few system related issues. 


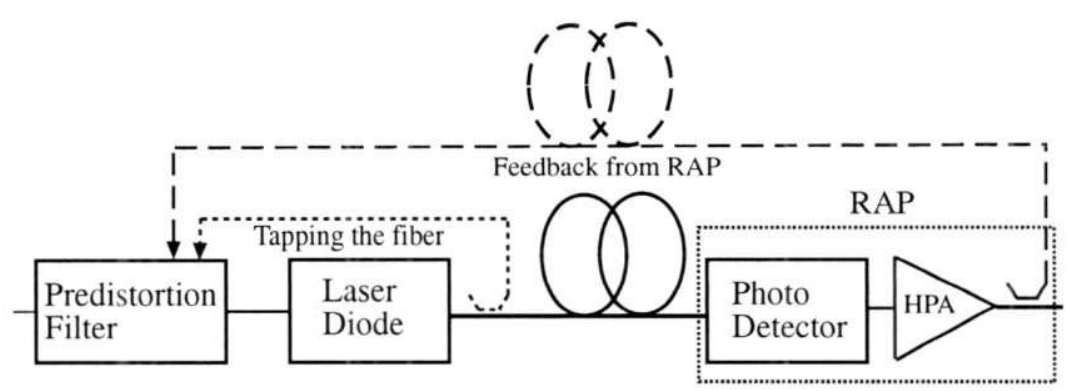

Figure 9.2: Different ways of obtaining feedback signal to the predistortion filter

\subsubsection{Memory of the Nonlinear System}

Initially, we assumed the optical link is memoryless. This is true up to few Mbps. When the sampling rate is high, the memory of the laser and matching circuits cannot be ignored. In this case, a suitable nonlinear model with memory has to be adapted. Furthermore, we have considered RF power changes smoothly. In practice, sudden RF power changes induce transients [11]. Tracking ability of the filters under transient conditions has to be explored. Usually, modeling and compensation of transient behavior is difficult and involves short-term memory issues.

Furthermore, fiber dispersion is significant at higher radio frequencies. In digitally modulated optical links, fiber dispersion introduces ISI. In analog links, dispersion introduces sideband cancellation. Optical single sideband modulation schemes have been investigated to solve the sideband cancellation issue [5]. However, this requires costly external modulators and delicate optical phase modulation schemes. There are unresolved issues, still in this area. Considerable chromatic dispersion means the fiber also has memory. Baseband modeling of chromatic dispersion is an interesting challenge. In this situation, the combined channel is very complicated. It has many linear dispersive systems (fiber, wireless channel, transmit, receive filters and matching circuits) and static nonlinear systems (laser and RF amplifier). A simple Wiener or Hammerstein model is inadequate. Models based on the cascade of these systems need to be explored. 


\subsubsection{Training the Predistortion Filter}

In chapter 4, we assumed that the desired response for training the predistortion filter is received at the central base station without distortion. This issue needs further investigation. The feedback can be provided in different ways, as shown in Fig. 9.2:

Tapping the Fiber: In this case, the fiber is tapped immediately after the optical transmitter to obtain the feedback signal. Wireless channel dispersion is not a concern here. Issues with this approach are:

1. Nonlinearity of the RF amplifier (HPA) at the optical receiver is not accounted for

2. The requirement of additional linear $\mathrm{O} / \mathrm{E}$ and $\mathrm{RF}$ to baseband conversion.

3. Additional power loss in the optical link because of the tapping.

Feedback from the RAP: The feedback can also be from the RAP. In this case, the wireless channel is not involved. Issues with this approach are:

1. ROF links are several kilometers long. Therefore, the feedback path also may be through another ROF (up) link, which would introduce additional distortion. Uplink compensation may be done first to avoid distortion in the feedback path.

2. This approach increases the complexity of the RAP.

Feedback from the portable: In this case the feedback signal undergoes both multipath dispersion and nonlinear distortion, therefore, not very desirable However, this is still possible if coefficients of the HDFE are first optimized.

\subsubsection{Additional Issues of the Optical Link}

In this work, the focus is on AM-AM and AM-PM type nonlinear distortions of the ROF link. There are other concerns in the ROF link, which need further investigation. For instance, for empirical studies we considered directly modulated ROF 
links. An interesting problem is to study the distortions in externally modulated links to see how different they are.

\subsubsection{Laser Chirp}

When E/O conversion is done by directly modulating a laser diode, there will be chirp distortion, which is an unwanted frequency modulation. Chirp is discussed in detail in section 2.1.6. When the chromatic dispersion of the fiber is significant, chirp would be converted into amplitude modulation and distort the RF output. Effects of the chirp involve input RF power, fiber dispersion and laser linewidth characteristics. Developing a suitable model to study the effects of chirp would be quite an interesting task.

\subsubsection{Clipping Distortion}

Depending on the instantaneous amplitude of the RF signal and the value of DC bias current, output optical signal gets clipped when the negative peak is going below the threshold. This issue is discussed in Section 2.1.2. Lai et al. [35] have done a good analysis on clipping noise. The clipping noise can become more critical when the optical modulation $m$ is high and in a multicarrier environment. Note that $m$ can be high with our proposed compensation schemes. Suitable coding techniques may reduce the clipping rate. This topic needs further investigation.

\subsubsection{System Related Issues}

A fiber based microcellular system has the following characteristics: Many weak inter-cell users interfere with each other because adjacent cells are too close. Few users interfere within the same cell (intra-cell), but they are strong. Yet, there is less near-far problem. Due to the small number of users, a Gaussian approximation to model the interference is generally not accurate. Noise level is relatively high, due to the optical link and the wireless channel. There will be residual nonlinearity of the optical link. These factors make this system different from a typical macro cellular environment and, need special attention in system related investigations. 


\subsubsection{Multiple PN Sequence Issue}

In our channel estimation algorithm, we assumed only a single PN sequence is transmitted. This is a special case. Typically, in CDMA systems, a summation of multiple PN sequences is transmitted. Therefore, interference from other PN sequences needs to be removed before using our algorithm for channel estimation. This issue needs further investigation.

\subsubsection{Multiple Cell Issue}

In this work, just point-to-point ROF links are considered. In reality, the individual fibers may be interconnected as a bus network, or as a star network or an hybrid of these two schemes. Additional investigation is necessary in this scenario to understand and compensate the distortion of these microwave fiber optic networks. This will give a significant challenge. 


\section{References}

[1] H. Zaghloul, G. Morrison, and M. Fattouche, "Frequency response and path loss measurements of indoor channel," Electronic Letters, vol. 27, no. 12, pp. 1021-1022, June 1991.

[2] S. Ohmori, Y. Yamao, and N. Nakajima, "The future generations of mobile communications based on broadband access technologies," IEEE Communications Magazine, vol. 38, no. 12, pp. 134-142, December 2000.

[3] Theodore S. Rappaport, Wireless Communications, Principles and Practice, Prentice Hall Inc., Upper Saddle River, NJ, 1996.

[4] Mark R. Gibbard, "Asymmetric equalization of the indoor radio channel," M.S. thesis, University of Calgary, 1996.

[5] Robert Davies, Optical Single Sideband for Broadband and Subcarrier Systems, Ph.D. thesis, University of Alberta, 2000.

[6] G.K. Gopalakrishnan, R.P. Moeller, M.M. Howerton, and R.D. Esman, "A low loss downconverting analog fiber-optic link," IEEE Transactions on Microwave Theory and Techniques, vol. 43, no. 9, pp. 2318-2323, September 1995.

[7] W. I. Way, "Subcarrier multiplexed lightwave system design considerations for subscriber loop applications," Journal of Lightwave Technology, vol. 7, no. 11, pp. 1806-1818, November 1989.

[8] Makoto Shibutani, Wataru Domon, and Katsumi Emura, "Reflection induced degradations in optical fiber feeder for micro cellular mobile radio systems," IEICE transactions on electronics, vol. E76-C, no. 2, pp. 287-291, February 1993. 
[9] Winston I. Way, "Optical fiber based microcellular systems: An overview," IEICE Transactions on Communications, vol. E76-B, no. 9, pp. 1091-1102, September 1993.

[10] J.C. Fan, C.L. Lu, and L.G. Kazovsky, "Dynamic range requirements for microcellular personal communication systems using analog fiber optic links," IEEE Transaction on Microwave Theory and Techniques, vol. 45, no. 8, pp. 1390-1397, August 1997.

[11] F. Cheng, P. Lemson, J.H. Reed, and Ira Jacobs, "A dynamic range enhancement technique for fiber optic microcell radio systems," in IEEE Vehicular Technology Conference (VTC 95), Illinois, 1995, pp. 774-778.

[12] Charles Cox III, Edward Ackerman, Roger Helkey, and Gary E. Betts, "Techniques and performance of intensity-modulation direct detection analog optical links," IEEE Transactions on Microwave Theory and Techniques, vol. 45, no. 8, pp. 1375-1383, August 1997.

[13] Rodney S. Tucker, "High speed modulation of semiconductor lasers," Journal of Lightwave Technology, vol. LT-3, no. 6, pp. 1180-1192, December 1985.

[14] L.S. Fock and R.S. Tucker, "Simultaneous reduction of intensity noise and distortion in semiconductor laser by feed forward compensation," Electronic Letters, vol. 27, no. 14, pp. 1297-1298, July 1991.

[15] H. Gysel and M. Ramachandran, "Electrical predistortion to compensate for the combined effects of laser chirp and fiber dispersion," Electronic Letters, vol. 27, no. 5, pp. 421-423, February 1991.

[16] Raziq Pervez and Masao Nakagawa, "Semiconductor laser's nonlinearity compensation for DS-CDMA optical transmission system by post nonlinearity recovery block," IEICE Transactions on Communications, vol. E-79 B, no. 3, March 1996.

[17] P. Vankwikelberge et al., "Analysis of the carrier induced FM response of the DFB lasers," IEEE Journal on Quantum Electronics, vol. 25, pp. 239-2254, November 1989. 
[18] David M. Cutrer, John B. Georges, T.H. Le, and K.Y. Lau, "Dynamic range requirements for optical transmitters in fiber fed microcellular networks," IEEE Photonic Technology Letters, vol. 7, no. 5, pp. 564-566, 1995.

[19] Wei Huang, Essam A. Sourour, and Masao Nakagawa, "Cancellation technique used for DS-CDMA signal in nonlinear optical links," IEICE Transactions on Fundamentals, vol. E80-A, no. 9, pp. 1616-1624, September 1997.

[20] Juin-Hung Chen and Hen-Wai Tsao, "FM sub carrier fiber optical transmission system design and its application in next generation wireless access," Journal of Lightwave Technology, vol. 16, no. 7, pp. 1137-1147, July 1998.

[21] H.S. Al-Raweshidy, D. Galbirth, and R. Prasad, "Performance of radio on fiber for microcellular GSM networks," in IEEE Conference on Vehicular Technology, 1998, pp. 392-396.

[22] Satoshi Kajiya, Katsutoshi Tsukumoto, and Shozo Komaki, "Proposal of fiberoptic radio highway networks using CDMA methods," IEICE Transactions of Electronics, vol. E79-C, no. 1, pp. 111-117, January 1996.

[23] Xavier Fernando and Abu Sesay, "Higher order adaptive filter characterization of microwave fiber optic link nonlinearity," in Proceedings of the SPIE, The International Society of for Optical Engineering, San Jose, January 2000, vol. 3927-06, pp. 39-49.

[24] Xavier Fernando and Abu Sesay, "Look-up table based adaptive predistortion for dynamic range enhancement in a radio over fiber link," in Proceedings of the IEEE Pacific Rim Conference on Communications, Computers and Signal Processing, Victoria, Canada, August 1999, pp. 26-29.

[25] Xavier Fernando and Abu Sesay, "Higher order adaptive filter based predistortion for nonlinear distortion compensation of radio over fiber links," in Proceedings of the IEEE International Conference on Communications ICC'00, New Orleans, June 2000, vol. 1, pp. 367-371.

[26] Xavier Fernando and Abu Sesay, "Nonlinear distortion compensation of microwave fiber optic links with asymmetric adaptive filters," in Proceedings of 
the IEEE International Microwave Symposium, Boston, June 2000, vol. 3, pp. $1821-1824$

[27] Xavier Fernando and Abu Sesay, "Adaptive asymmetric linearization of radio over fiber links for wireless access," to be published in IEEE Transactions on Vehicular Technology.

[28] Norbert Wiener, Nonlinear Problems in Random Theory, Number QA401 W5. Technology press of MIT and John Wiley and Sons Inc. New York, 1958.

[29] D. V. Sarwate and M. B. Pursley, "Crosscorrelation properties of pseudorandom and related sequences," Proceedings of the IEEE, vol. 68, no. 5, pp. 593-619, May 1980.

[30] S. A. Billings and S. Y. Fakhouri, "Identification of nonlinear systems using correlation analysis of pseudorandom inputs," International Journal of Systems Science, vol. 11, no. 3, pp. 261-279, 1980.

[31] Xavier Fernando and Abu Sesay, "Nonlinear channel estimation using correlation properties of PN sequences," in Proceedings of the Canadian Conference on Electrical and Computer Engineering, Toronto, ON, Canada, May 2001, pp. 469-474, First prize winner of the student paper competition.

[32] Xavier Fernando and Abu Sesay, "A Hammerstein type DFE for the fiberwireless uplink," in Proceedings of Wireless-2001, The 12th International Conference on Wireless Communications, July 2001, vol. 1, pp. 318-325.

[33] W. I. Way, "Large signal nonlinear distortion prediction for a single-mode laser diode under microwave intensity modulation," Journal of Lightwave Technology, vol. LT-5, no. 3, pp. 305-315, March 1987.

[34] C. O. Weiss and R. Vilaseca, Dynamics of Lasers, VCH Publishers, 1991.

[35] Stephen Lai and Jan Conradi, "Theoretical and experimental analysis of clipping-induced impulsive noise and am-vsb subcarrier multiplexed lightwave systems," Journal of Lightwave Technology, vol. 15, no. 1, pp. 20-30, January 1997. 
[36] R. S. Tucker and T.E. Darcie, "Intermodulation and harmonic distortion in InGaAsP lasers," Electronic Letters, vol. 21, no. 16, pp. 665-666, August 1985.

[37] Michel C. Jeruchmin et al., Simulation of Communication Systems, Plenum Press, New York, 1992.

[38] C.D. Poole and T.E. Darcie, "Distortion related to polarization-mode dispersion in analog lightwave systems," Journal of Lightwave Technology, vol. 11, no. 11, pp. 1749-1759, November 1993.

[39] Louis Moura, Matthew Darby, Phil Lane, and John O'Reilly, "Impact of interferometric noise on the remote delivery of optically generated millimeter-wave signals," IEEE Transactions on Microwave Theory and Techniques, vol. 45, no. 8, pp. 1398-1402, August 1997.

[40] John M. Senior, Optical Fiber Communications: Principles and Practice, Number TK5103.59 .S46. Prentice Hall -, 2 edition, 1992.

[41] Microwave Fiber Optics Group, "A system designer's guide to RF and microwave fiber optics," Tech. Rep., Ortel Corporation, 1999.

[42] Goran Einarsson, Principles of Lightwave Communications, Number TK 5103.59 E.36. John Wiley and Sons, West Sussex, England, 1995.

[43] John Tsimbinos and Kenneth V. Lever, "Input nyquist sampling suffices to identify and compensate nonlinear systems," IEEE Transactions on Signal Processing, vol. 46, no. 10, pp. 2833-37, October 1998.

[44] R.J. Martin, "Volterra system identification and kramer's sampling theorem," IEEE Transactions on Signal Processing, vol. 47, no. 11, pp. 3152-3155, November 1999.

[45] Y. M. Zhu, "Generalized sampling theorem," IEEE Transactions on Circuits and Systems-II, vol. 39, pp. 587-588, August 1992.

[46] A. A. M. Saleh, "Frequency independant and frequency dependant nonlinear models of TWT amplifiers," IEEE Transactions on Communications, vol. COM-28, pp. 1715-1720, November 1981. 
[47] Simon Haykin, Adaptive Filter Theory, Prentice Hall Inc., Englewood Cliffs, NJ, 1991.

[48] W. Bosch and G. Gatti, "Measurement and simulation of memory effects in predistortion linearizers," IEEE Transactions on Microwave Theory and Techniques, vol. 37, no. 12, pp. 1885-1890, December 1989.

[49] A. A. M. Saleh and R. A Valenzula, "A statistical model for indoor multipath propagation," IEEE Journal on Selected Areas in Communications, vol. 5, no. 2, pp. 128-137, February 1987.

[50] John G. Proakis, Digital Communications, McGraw Hill Inc, New York, NY, 1995.

[51] Martin Schetzen, "Nonlinear system modeling based on the Wiener theory," Proceedings of the IEEE, vol. 69, no. 12, pp. 1557-1573, December 1981.

[52] L. AuthorSutek and M. Varga, "Identification of nonlinear systems through the utilisation of discontinuous orthogonal filters in applying a multilevel pseudorandom signal," in Proceedings of the 3rd IFAC Symposium on Identification and System Parameter Estimation, North Holland, Amsterdam, Netherlands, Nov 1973, vol. 12, pp. 839-42.

[53] R. Haber and H. Unbehauen, "Structure identification of nonlinear dynamic systems - a survey on input/output approaches," Autamatica, vol. 26, no. 4, pp. 651-677, 1990.

[54] M. Korenberg, S.A. Billings, Y.P. Liu, and P.J. McIlroy, "Orthogonal parameter estimation algorithm for nonlinear stochastic systems," International Journal of Control, vol. 48, no. 1, pp. 193-210, 1988.

[55] S. A. Billings and S. Y. Fakhouri, "Identification of systems containing linear dynamic and static nonlinear elements," Automatica, vol. 18, no. 1, pp. 15-26, 1982 .

[56] N. J. Bershad, S. Bouchired, and F. Castanie, "Stochastic analysis of adaptive gradient identification of Wiener-Hammerstein systems with Gaussian inputs," 
IEEE Transactions on Signal Processing, vol. 48, no. 2, pp. 557-560, February 1999.

[57] G. T. Zhou and G. B. Giannakis, "Nonlinear channel identification and performance analysis with PSK inputs," in Signal Processing Advances in Wireless Communications, 1997, pp. 337-340.

[58] Kurt Metzger and Rolf Valentin, "Intersymbol interference due to linear and nonlinear distortion," IEEE Transactions on Communications, vol. 44, no. 7, pp. 809-815, July 1996.

[59] S. A. Billings and S. Y. Fakhouri, "Identification of nonlinear systems using the Wiener model," Electronic letters, vol. 13, no. 17, pp. 502-504, August 1977.

[60] Yonathan Bard, Nonlinear Parameter Estimation, Number QA 276.8 B37. Academic Press New York and London, IBM Corporation, Cambridge, Massachusetts, 1974 .

[61] John G. Proakis, "Adaptive equalization for TDMA digital mobile radio," IEEE Transactions on Vehicular Technology, vol. 40, no. 2, pp. 333-341, May 1991.

[62] D. G. Lainiotis and P. Papaparaskeva, "A partitioned adaptive approach to nonlinear channel equalization," IEEE Transactions on Communications, vol. 46, no. 10, pp. 1325-1336, October 1998.

[63] S. Chen, G.J. Gibson, C.F.N. Cowan, and P. M. Grant, "Adaptive equalization of finite nonlinear channels using multilayer perceptrons," IEE Proceedings on Signal Processing, pp. 107-119, November 1989.

[64] S. Ozgunel, A.H. Kayran, and E. Panayirci, "Nonlinear channel equalization using multichannel adaptive lattie algorithm," in IEEE International Symposium on Circuits and Systems, 1991, pp. 2826-29.

[65] S. Chen and Y. Wu, "Maximum likelihood joint data and channel estimation using genetic algorithms," IEEE Transaction on Signal Processing, vol. 46, no. 5, pp. 1469-1473, May 1998. 
[66] C. A. Belfiore and J. H. Park Jr., "Decision feedback equalization," Proceeding of the IEEE, vol. 67, no. 8, pp. 1143 -1156, August 1979.

[67] D.D. Falconer, "Adaptive equalization of channel nonlinearities in QAM data transmission systems," Tech. Rep. 7, Bell Systems Technical Journal, September 1978 .

[68] W. Frank and Ulrich Appel, "Efficient equalization of nonlinear communication channels," in Proceedings of the IEEE International conference on Acoustics, Speech and Signal Processing, Germany, April 1997, vol. 3, pp. 2501-2504.

[69] D. M. Topalovic, "Adaptive lattice equalizer to compensate nonlinearities of digital radio channels with MPSK modulation," in IEEE 9th Mediterranean Electrotechnical Conference, NY, USA, 1998, vol. 1/2, pp. 617-21.

[70] Jui-Yuan Lin and Che-Ho Wei, "A new adaptive equalizer for nonlinear channels," IEEE International Symposium on Circuits and Systems, vol. 5/5, pp. 2814-17, June 1991.

[71] K. Y. Lee, "Fuzzy adaptive decision feedback equalizers," Electronic Letters, vol. 30, no. 10, pp. 749-751, May 1994.

[72] S. Siu, G.J. Gibson, and C.F.N. Cowan, "Decision feedback equalizers using neural network structures and performance comparison with standard architectures," IEE Proceeding Part I, vol. 137, no. 4, pp. 221-225, August 1990.

[73] M. Meyer and G. Pfeiffer, "Multilayer perceptron based decision feedback equalisers for channels with inter symbol interference," IEE Proceedings - Part I, vol. 140, no. 6, pp. 420-424, December 1993.

[74] F.J. Gonzalez-Serrano, F. Perez-Cruz, and A. Artez-Rodriguez, "Reducedcomplexity equaliser for nonlinear channels," Electronic Letters Online No. 19980618, March 1998.

[75] F.J. Gonzalez-Serrano, F. Perez-Cruz, and A. Artez-Rodriguez, "GCMAC based equaliser for nonlinear channels," Proceedings of the IEEE International conference on Acoustics, Speech and Signal Processing, vol. 2/6, pp. 1165-8, 1998. 
[76] V.J. Mathews and Jungshi Lee, "Adaptive algorithms for bilinear filtering," in The International Society of Optical Engineering SPIE, San Diego, CA, 1994, vol. 2296, pp. 317-327.

[77] Shahid U. H. Qureshi, "Adaptive equalization," Proceedings of the IEEE, vol. 73, no. 9, pp. 1349-1387, September 1985.

[78] John E. Smee and Norman C. Beaulieu, "On the equivalence of the simultaneous and separate MMSE optimization of a DFE FFF and FBF," IEEE Transactions on Communications, vol. 45, no. 2, pp. 156-158, February 1997.

[79] N. Al-Dhahir and J. M. Cioffi, "MMSE decision feedback equalizers: Finitelength results," IEEE Transactions on Information Theory, vol. 41, no. 4, pp. 961-975, July 1995.

[80] Yow-Jong Liu, Mark Wallace, and Ketchum John W., "A soft-output bidirectional decision feedback equalization techniques for TDMA cellular radio," IEEE Journal on Selected Areas in Communications, vol. 11, no. 7, pp. 10341045, September 1993.

[81] J. Salz, "Optimum mean-square decision feedback equalization," Tech. Rep. 8, Bell System Technical Journal, October 1973.

[82] Michel J. Eric, "Intermodulation analysis of nonlinear devices for multiple carrier inputs," Tech. Rep., Communication Research Center, Canada, 1993. 


\section{Appendix A}

\section{Baseband Representation of a Passband Nonlinearity}

Communications signals and systems are passband. Appropriate translation of these into baseband is required to use baseband signal processing. This translation is not straightforward when nonlinear systems are involved. This issue is discussed in this appendix where it shown that a bandpass nonlinear system can be either represented by two real orthogonal baseband nonlinear models or by an amplitude and phase baseband nonlinear model.

\section{A.1 Bandpass Nonlinear Distortion}

When an information bearing RF signal undergoes a nonlinear transform, two different types of distortions occur.

Carrier Regrowth Additional frequency components are generated due to the nonlinearity. This is a major concern in multicarrier environments since the cross modulation products would overlap. Even with single carriers, RF design engineers struggle to minimize the harmonics due to carrier re-growth, to meet FCC regulations.

Inband Distortion Due to the amplitude and phase distortion of the vector modulated symbols, well known AM-AM and AM-PM distortions occur. When the nonlinearity has no memory, these distortions produces a 'warped' con- 
stellation, instead of the original rectangular grid constellation, similar to the one shown in Fig. 2.7 in page 41. This increases the BER especially with QAM type modulations.

In addition, when the memory of the nonlinearity is comparable to the sampling rate, each point of the signal constellation becomes a 'cluster' which reflects intersymbol interference (ISI) at the sampling instant. This is a more complex form of inband nonlinear distortion.

The inband distortion is our major concern in this work. This exists in both single and multi carrier transmission, even with spread spectrum cases.

\section{A.2 Inband Nonlinear System}

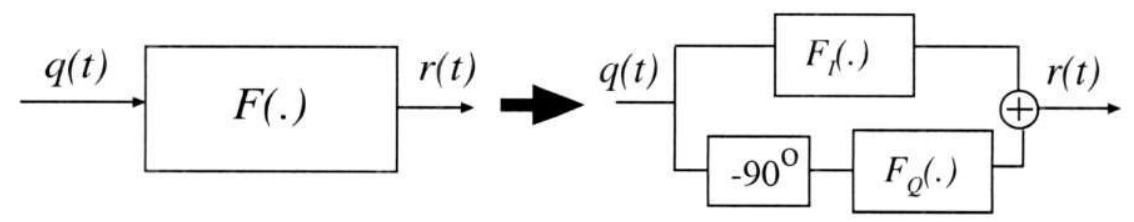

Figure A.1: Inphase and Quadrature Representation of a Nonlinear System

A bandpass nonlinear system is shown in Fig. A.1 (left). It introduces both AM-AM and AM-PM distortions. Let the input signal $q(t)$ is given by,

$$
q(t)=A(t) \cos \left[\omega_{c} t+\theta(t)\right]
$$

Then, the output $r(t)$ is,

$$
r(t)=R[A(t)] \cos \left\{\omega_{c} t+\theta(t)+\phi[A(t)]\right\}
$$

where, $R$ is the amplitude of $r(t)$ and $\phi$ is the phase shift of $r(t)$ compared to the input phase. These are functions of the input amplitude. This also can be expressed as,

$$
r(t)=r_{p}[A(t)] \cos \left[\omega_{c} t+\theta(t)\right]-r_{q}[A(t)] \sin \left[\omega_{c} t+\theta(t)\right]
$$


using the trigonometric identity $\cos (A+B)=\cos (A) \cos (B)-\sin (A) \sin (B)$. Here, $r_{p}$ and $r_{q}$ are the inphase and quadrature amplitude distortions. These are related to $R$ and $\phi$ by,

$$
\begin{aligned}
& r_{p}[A(t)]=R[A(t)] \cos \{\phi[A(t)]\} \\
& r_{q}[A(t)]=R[A(t)] \sin \{\phi[A(t)]\}
\end{aligned}
$$

Both of these inphase and quadrature components are linearly independent for an arbitrary input signal. Therefore, their power spectral densities can be added.

Note that, in equation (A.3), the bandpass nonlinearity is expressed in two simple orthogonal amplitude nonlinearities by a projection onto a rectangular coordinate system. The equivalent block diagram is shown in Fig. A.1 (right). Similarly, a bandpass nonlinearity can also be projected in to a polar coordinate system as shown in [82] so that, there are again two channels. These two methods are compared below:

\section{Rectangular Form}

- Both inphase and Quadrature Components are only amplitude distorted The phase distortion, however, is taken care by the vectorial sum at the output.

- These amplitude distortions are functions of only the amplitude of the input signal.

The obvious advantage of the rectangular form is that it allows us to deal with only amplitude nonlinearities. That is, we deal with real nonlinear models rather than complex models. Therefore, this form is used in the latter part of this thesis. That is, in Wiener and Hammerstein type system modeling and compensation. 


\section{Polar Form}

- Each channel introduces both amplitude and phase distortion.

- The phase distortion is additive and the amplitude distortion is multiplicative.

- Both of these distortions are functions of only the amplitude of the input signal.

The advantage of the polar form is that we have an additive phase correction. Therefore, this approach is used in baseband modeling, predistortion and postcompensation work of this thesis. 